

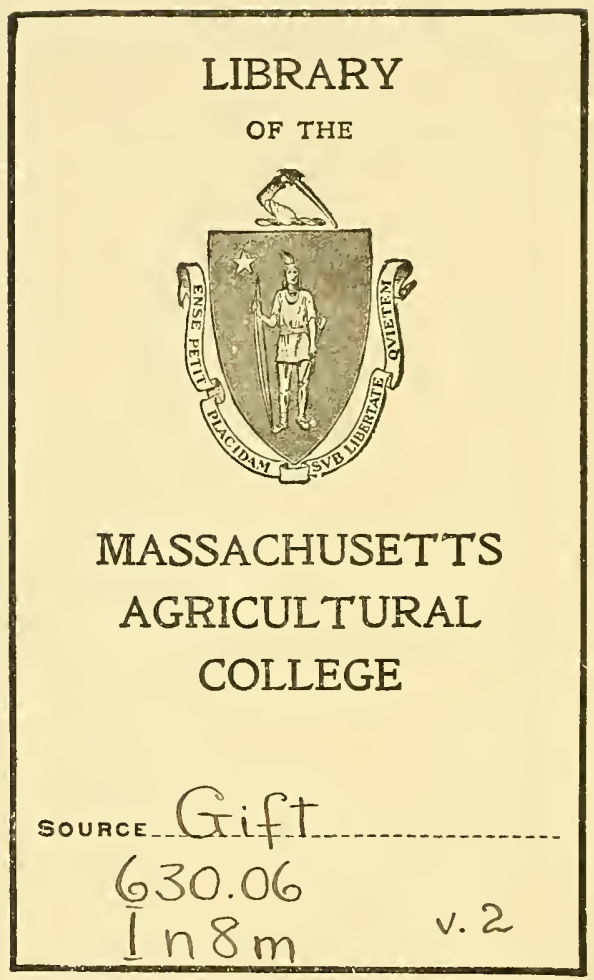

CHAPEL 
This book may be kept out

TW

only, and is su

CENTS a day thi

the day indicated

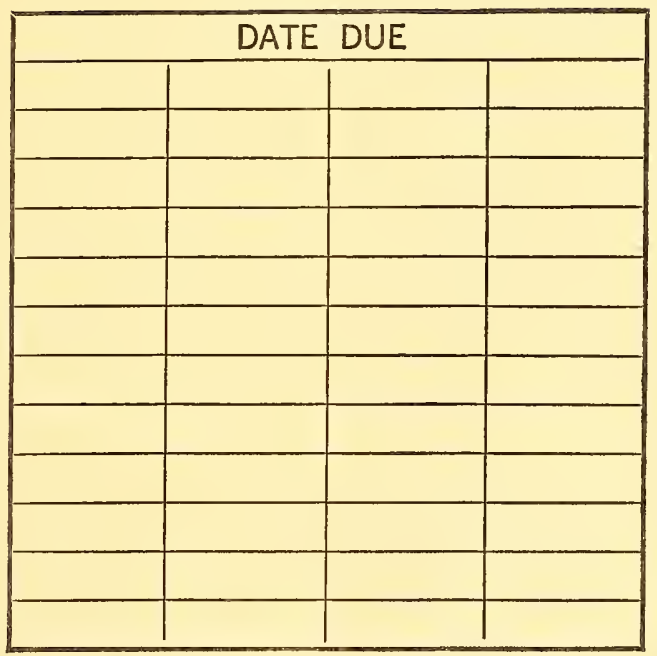

1429

A4

1910

V.2 



INTERNAIIOIAI INGTITUTE OF AGRICUITURE

\section{MISCHILAHEOUS PUBIICATIUIS}

Volume ?

$$
41
$$


Digitized by the Internet Archive in 2011 with funding from

Boston Library Consortium Member Libraries 


\section{INSTITUT IN'TERNATIONAI， D'AGRICULTURE}

BUREAU DES RENSEIGNEMENTS AgRICOLES ET DES MaLAdIHS DES PLANTES

\section{LA LUTTE CONTRE LES SAUTERELLES}

\section{DANS LES DIVERS PAYS}

\section{ROME}

MPRIMERIE DE L'INSTITUT INTERNATIONAL D'AGRICULTURE 


\section{Publications de l'Institut International d'Agriculture}

\section{A. PUBLICATIONS MENSUELLES.}

I. BULletin de statistique agricole et commerciale 'publié chaque mois en français, allemand, anglais, espagnol et italien, in-16) . . .

2. BULLETLY MENSUEL DES RENSEIGNEMENTS AGRTCOLES ET DES MALADIES DES PLANTES (eu français, allemand, anglais, espagnol et italien,

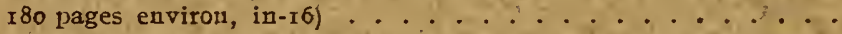

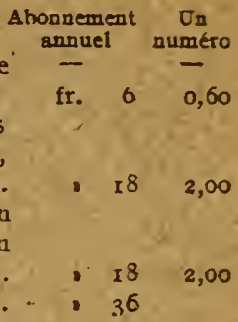

B. ANNUAIRES.

I. ANNUATRE INTERNATTONAL DE STATISTIQUE AGRICOLE POUR I9IO (I9I2,

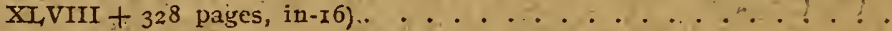
IDEM, pour I9II-I9I2 (I9I4, XXXIV +624 pages, in-I6) ….. IDEM, pour I9I3-19I4 (I9I5, XIIV + 788 pages, in-I6) …..

2. ANNCAIRE INTERNATIONAL DE LÉGISLATION AGRICOLE, I Pre ANAÉE, I 9 I I (I9I2,

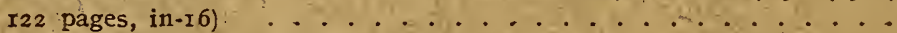
IDEM, z ane Année, rgr2 (I913, 994 pages, in-I6) . . . . . . .

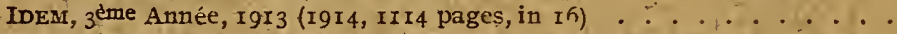
IDEM, $4^{\text {eme }}$ Année, I9I4 (I915, LXVIII + I020 pages, in-I6). ..... IDEM, 5 eme Annee, 1915 (1916, LXXXXVI + 1460 pages, in-I6) ....

C. AUTRES PUBLICATIONS. a) Publications de la Bibliothèque.

1. CATALOGUE DE LA BibliothèQUE. Année I $909(356$ pages, in-8) . . .

2. LISTE DES REVUES ET JOURNAUX RÉGULIEREMENT RECUUS PAR L'INSTITUT. Année rgr $3(84$ pagés, in-r6) $\ldots \ldots \ldots \ldots \ldots$

b) Publications du Bureau de la Statistique Gènérale.

$$
\text { I) - Publications diverses: }
$$

I. L'ORGANISATION DES SERVICES DE STATISTIQUE AGRICOLE DANS LES DIVERS pays (Tome I) (Igro, 446 pages, in-I 6 , avec tableaux hors texte) : . . IDEM (Tome II) (I9I 3, I46 pages, in-I6) $\ldots \ldots \ldots \ldots \ldots$

2. RECUEIL DE COEFFICEENTS POUR LA CONVERSION DES POIDS, MESURES ET MONNATES AU SYSTĖME MÉTRIQUE DÉCIMAL (1914,84 pages,in-32) . . . . .

fr. 4,00

or 2,00

- 1,00

\section{2) - Monographies (Nourelle série).}

I. L'ORGANISATION DE LA STATISTIQUE DU COMMERCE EXTÉRIEUR EN ITALIE (I9r3, Igo pages, in-16).

2. LE MARChÉ DES CÉREALES D'ANVERS (1913,62 pages,in-16) . . . . . .

3. LES BOURSE'S DES PRODUITS AGRICOLES DE HAMBOURG ET BUDAPEST (I9I3, 55 pages, in-16)

4. NOTES SUR LES STATISTIQUES DU COMMERCE EXTÉRTEUR DANS LES DIFFERENTS PAYs :-Publications statistiques, ierritoires, sortes de commerce, provenances et destinations des marchandises ( $19 \mathrm{r}_{4}, 96$ pages, in-16) $\ldots \ldots \ldots$

5. RÉPARTITION AGRICOLE DES TERRITOIRES DES DIFFÉRENTS PAYS (IOI 4 , 3 IO pages, in-I6).

\section{3)-Autres publications:}

UMBERTo Ricci. - Les bases théoriques de la statistique agricole internationale $\left(\mathrm{r}^{2} \mathrm{I}_{4}, 3 \mathrm{I} 4\right.$ pages, in-I6)

c) Publications du Bureau des Renseiguements Agricoles et des ILaladies des Plantes.

I. LE SERVICE DE PROTECTION CONTRE LES MALADIES DES PLANTES ET LES INSECTES NUISIBLES DANS LES DIVERS PAYS (rǵr 4,350 pages, in-4) ....

2. PRODUCTION ET, CONSOMMATION DES ENGRAIS CHIMUUES DANS LE MONDE fr. 4,00 ( $2^{\circ}$ édition, IgI4, I62 pages, 5 diagrammes, 2 cartes, in-16) 


INSTITU'T INTERNA'TIONAL D'AGRICULTURE

Bureau des Rfanghignemifnts Agricoles e't des Maladies des Plantils

\section{LA LUTTE CONTRE LES SAUTERELLES}

\section{DANS LES DIVERS PAYS}

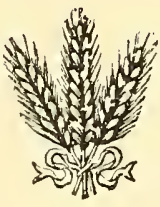

ROME

IMPRINERIE DE L'INSTITUT INTERNATIONAL D'AGRICULTURE 



\section{INTRODUCTION}

A la distance de trois ans, deux personnalités compétentes ont invoqué l'aide de l'Institut International d'Agriculture dans la solution d'un problème d'une importance vitale pour l'agriculture de plusieurs pays: la lutte contre les Sauterelles.

Monsieur I. BaLdRATI, Directeur de l'Office de Colonisation de l'Erythrée, en signalant les ravages qu'occasionnent ces redoutables orthoptères dans la Colonie italienne, montrait à l'Institut le grand service qu'il rendrait à l'Agriculture s'il lui était possible de faire connaître, dans un rapport aussi complet que détaillé, tout ce qu'on fait dans les divers pays du monde pour lutter contre ce fléau. M. BAIDRATI demandait "une publication spéciale résumant l'état de fait»; il faisait, en même temps, allusion à l'utilité qu'il y aurait à provoquer un "Congrès des Sauterelles".

Dernièrement, Monsieur MALET, Directeur de l'Agriculture, du Commerce et de la Colonisation dans le Protectorat français du Maroc, dans le même ordre d'idées, proposait résolument à l'Institut de prendre l'initiative d'une Conférence internationale appelée à examiner à fond la question, et à proposer érentuellement les mesures de nature internationale susceptibles de rendre la lutte plus efficace, l'application de mesures locales et isolées n'ayant toujours qu'une portée circonscrite et insuffisante. Il faisait remarquer, notamment, qu' "il faudrait déterniner soigneusement les régions permanentes d'où partent les invasions, et se livrer, dans ces régions, à une lutte systématique \%。

Cette conformité de vues de personnalités appelées à sauvegarder les intérêts agricoles de pays sujets aux attaques fréquentes d'un ennemi commun est une preuve que, malgré les efforts des savants et des administrateurs des intérêts publics, les movens de lutte essayés 
depuis de longues années sont encore loin d'assurer dans tous les cas la victoire: il existe toujours une "question des Sauterelles".

L'Institut International d'Agriculture - dont la haute mission est précisement celle de se servir des moyens étendus dont seule une institution d'Etat internationale est susceptible de disposer, pour aider à la solution de toutes les questions intéressant l'agriculture de: divers pays du monde - saisit avec empressement la proposition de l'Office de 1'Erythrée: aussitôt, le Conité Permanent chargeait le Service des Renseignements agricoles et des Maladies des Plantes d'ouvrir une enquête internationale en vue d'obtenir les éléments nécessaires pour rédiger le Rapport demandé. Dès ce moment, ce Service se fit un devoir de se mettre à l'œuvre et il s'empressa d'adresser aux divers Eitats un questionnaire adéquat.

En fornulant ce questionnaire, nous ne nous sommes pas bornés aux demandes relatives aux seuls moyens de lutte employés et aux résultats obtenus; nous avons estimé utile et nécessaire de demander, en même temps, des renseignements détaillés sur la biologie des insectes qui nous occupent; nous nous sommes inspirés, ce faisant, d'un principe qu'on ne saurait jamais négliger sans tomber dans les risques et les erreurs qui caractérisent l'empirisme : dans la solution de tout problème concernant un être vivant, la connaissance parfaite de sa biologie est à considérer comme une conditio sine qua non.

Cette connaissance est le seul moyen d'atteindre le but en suivant le chemin de la Science selon les préceptes préconisés il y a déjà longtemps par LEONARDO DA VINCI et de l'atteindre en temps dû, d'après la maxime de Claude Bernard : "Prévoir et agir ".

Comme tous les ennemis de l'agriculture qui attaquent les plantes en hordes innombrables, il importe de s'attacher à les supprimer arant qu'ils se soient développés à tel point que la lutte soit rendue partieilement efficace sinon même impossible : de là, la nécessité de connaître l'insecte dès le début de sa vie, de manière à pouroir le supprimer dans son premier foyer, les lieux de ponte.

Malheureusement, il n'a pas été toujours facile ou possible, jusqu'à présent, d'employer cette méthode prophylactique: les invasions en masses immenses en sont une preuve. De là, la nécessité de connâ̂tre avec la plus grande précision, non seulement 1'habitat des orthoptères dévastateurs et les terrains de ponte, mais encore leur cycle évolutif, les modalités des apparitions, le mécanisme des migra- 
tions et la direction des vols, leur nourriture préférée et les plantes dédaignées; d'en connaître les mœurs dans les moindres détails, pour être en mesure "d'agir» opportunément, en les attaquant au moment le plus propice, par les moyens les plus appropriés.

Toujours dans ce même ordre d'idées, il est nécessaire de connaître la biologie de ces insectes considérés dans leurs rapports avec leur entourage, afin de découvrir, notamment, leur degré de faiblesse vis-à-vis des maladies qu'ils sont susceptibles de contracter et des parasites qu'à l'instar de tout être vivant, ils sont capables d'héberger, les causes de l'impuissance de ceux-ci à rendre à l'agriculture les services remarquables que, dans d'autres cas, savent rendre ces organismes bienfaisants que nous comprenons sous le nom de parasites des parasites.

L'aperçu historique, qui constitue le premier chapitre de notre Rapport, permet de se faire une idée de la lutte contre les Sauterelles à travers les siècles. Ce n'est toutefois qu'aux alentours de I87o qu'on s'attache à donner à cette lutte une orientation nouvelle; on reconnaît, à cette époque, la nécessité de s'adresser à la Science pour obtenir ce que jusque là avait caché l'empirisme; à savoir les critères fondamentaux d'une organisation de la lutte basée sur la connaissance de l'habitat, de la biologie et des mœurs de l'ennemi.

Il est de toute justice de rappeler d'une façon toute particulière les précurseurs qui jetèrent les bases de cette organisation scientifique de la lutte: les naturalistes russes KöNCHSÈviTcH, KOPPEN et KRASSILSTCHIK - qui a trouvé les "foyers permanents" de multiplication de Pachytylus migratorius en Russie - ; les américains C.-V. RILEY, A.-S. PACKARD et Cyrus Thomas - qui établirent les trois grandes étapes des invasions: les "régions permanente, sub-permar.ente et $t \in m$ poraire " et qui réunirent dans un Rapport monumental une foule de constatations précieuses; le français KÜNCKEL D'HERCULAIS, qui s'attacha à parfaire l'œuvre de ses prédécesseurs et contemporains, tout en utilisant les lumières que leurs travaux avaient jetées sur la question.

Chargé par le Gouvernement français de se rendre en Algérie pour organiser un Service d'étude et de destruction des orthoptères dévastateurs, M. KüNCKEL, D' HERCULAIS y arrivait à un moment où - comme il nous le dit lui même dans son ouvrage magistral - on allait jusqu'à assimiler les Sauterelles aux météores, puisque dans la Colonie, c'était la direction du Service Météorologique qui était 
chargée de'renseigner sur les évolutions des invasions, "tout aussi bien quesur leurs moeurs ». Le même savant, lorsqu'il parle des conséquences fatales auxquelles menait l'ignorance des mours de l'ennemi, nous rappelle qu'il a dû combattre à outrance la croyance-dérivée de la légende arabe - que le stauronote marocain n'était qu'un descendant du criquet pèlerin; d'où des illations fantaisistes autant que dangereuses, qui menaient à conclure à l'extinction naturelle des invasions par abâtardissement de l'espèce.

Les théories, ou plutôt les croyances dont les, observations successives ont montré la fausseté ou l'inexactitude étaient par trop nombreuses: on confondait les espèces; on contestait la pluralité des pontes; on prêtait encore foi à l'assertion de MaHomet suivant laquelle les Sauterelles ne survivent pas à l'acte de la procréation; on ne faisait pas de différence entre espèces autochtones et espèces migratrices; on attribuait une valeur exagérée aux agents météorologiques, comme moyens naturels de destruction; et d'autres errements sont encore signalés par M. KÜNCKEL, D'HERCULAIS dans l'ouvrage qư'on trouvera plusieurs fois mentionné dans le présent Rapport et qui peut être, à juste titre, considéré comme un ouvrage classique autant pour la documentation que pour la solution des problèmes physiologiques qui doivent être la base fondamentale d'une organisation scientifique de la lutte. Cet ouvrage et les Rapports des naturalistes américains RILEY, PACKARD et CyRUs Thomas constituent aujourd'hui encore la base fondamentale de l'orientation rationnelle à donner à la lutte contre les déprédateurs qui nous occupent.

La littérature sur les orthoptères s'est enrichie par la suite des études faites par des naturalistes pour jeter de nouvelles lumières sur l'important problème; mais, à part quelques modifications dans le mode d'emploi, fondamentalement, les moyens de lutte sont toujours les mêmes, les résultats insuffisants.

Sur toutes les questions relatives à l'habitat et à la biologie des diverses espèces d'orthoptères déprédateurs et aux moyens de lutte employés, nous demandions - par notre questionnaire très détaillé des renseignements scientifiques, techniques et économiques basés sur l'observation directe locale, et nous demandions qu'ils nous fussent communiqués par les "personnalités compétentes" de chaque pays. Nous demandions également à être renseignés sur l'organisation de la lutte et sa réglementation éventuelle par des mesures législatires 
et administratives. Nous demandions, en outre, quelles étaient les propositions qu'il paraitrait opportun de faire dans chaque pays sur la base de l'expérience acquise et, en l'espèce, si une entente internationale semblerait susceptible de conduire à une meilleure solution de la question. Cela pour nous mettre en mesure de rassembler une do cumentation originale concernant des faits constatés sur place dans chaque cas; notre tâche n'étant pas celle d'écrire une véritable monographie des Sauterelles, mais de fournir des éléments précis pouvant servir à aider à la solution du problème qui motivait notre enquête.

Presque tous les Eitats qui ont plus ou moins à souffrir des invasion ont réservé un accueil favorable à notre demande. Un petit nombre seulement, n’ont pas encore pu répondre à notre questionnaire. Nous aurons soin de porter à la connaissance du public les rapports nouveaux qui pourront nous parvenir ultérieurement.

Nous pourrions considérer les réponses que nous ont fournies cent dix pays comme une documentation presque complète, si l'absence de certains rapports et en particulier de celui de la Russie, ne constituait pas une lacune assez notable. En ce qui concerne la Russie, ce pays, à en juger d'après ce que nous apprennent les publications russes possédées par notre Institut, est en mesure d'offrir une large moisson de connaissances précieuses, puisque la question des Sauterelles y fait toujours l'objet d'études et d'investigations nombreuses.

C'est le cas de rappeler ici les publications de M. B. Uvarov (I), Directeur de l'Office d'Entomologie agricole du Département de l'Agriculture à Stavropol, qui, après avoir donné un aperçu historique des invasions des Sauterelles en Russie, et en avoir indiqué les espèces nuisibles, leur localisation et tout ce que l'on connaît quant à leur biologie, s'est attaché à exposer la valeur des divers moyens de lutte employés. Sur ce dernier point, il y a lieu de retenir la conclusion à laquelle il serait parvenu, à savoir que seuls les moyens chimiques sont susceptibles d'assurer la victoire; parmi ceux-ci, la préférence devant être donnée

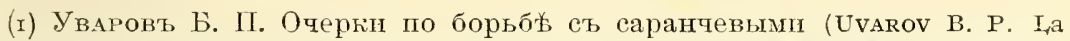

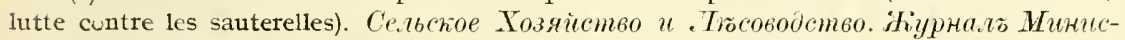
mepcm6a Зелледrõ $\lambda$, (L'A griculture et la Sylviculture. Revue du Ministère de l'A griculture). Pétrograd, février et mars rgr5, pp. 266-28I et 377-4I4; mai rgr6, pp. 3I-47.

Voir aussi: Instirut International D'AGRICULture. Bulletin mensuel des Renseignements agricoles et des Maladies des Plantes, No 66.4, juin I9I5 et No 1239, novembre r9I 6. 
au son arsenié, suivant la méthode américaine. Il estime que les moýens chimiques assureront un succès jusqu'à présent empêché par le manque d'une organisation convenable. Il impute le manque d'un succès complet à l'absence de liaison entre les diverses institutions entomologiques, qui travaillent chacune pour son propre compte, en employant souvent des moyens impropres. Ira nécessité d'une entente entre ces institutions a été reconnue au Congrès de Kiev, oì l'on a préconisé des réunions annuelles des entomologistes préposés à la lutte contre les Sauterelles, dans le but d'examiner ensemble les rapports des travaux effectués et d'élaborer le plan de ceux à effectuer l'année suivante.

Pour prouver d'une façon lumineuse les graves conséquences du manque de coordination dans les travaux de lutte - chaque province étant livrée isolément à ses propres moyens - il cite le cas des invasions de Stauronotus maroccanus et Pachytylus migratorius dans la province de Stavropol en IgII-IgI2. Par l'emploi de mesures énergiques et systématiques, on avait débarrassé cette province des deux insectes, mais ils y revinrent, en automne IgI2, de la région de Tersk et de la Province d'Astrakhan, par hordes immenses qui déposèrent leurs œufs sur une étendue de 22 ooo hectares. Les indiridus issus de l'éclosion de ces œufs furent détruits en I9I3, mais tune nourelle ponte eut lieu au mois d'août de la même année par de nourelles bandes venant encore de la région de Tersk.

M. Uvarov nous signale les progrès faits en Russie au sujet de la détermination des "régions permanentes" de l'ennemi et des causes de leurs déplacements. Il expose l'état actuel de l'organisation de la lutte, qu'il trouve insuffisante, et il préconise l'action directrice de l'Etat "qui devrait prendre l'initiative de créer des institutions entomologiques dans les régions qui représentent des foyers permanents de multiplication des Sauterelles, d'où elles se répandent dans les régions contiguës \%. Des mesures législatives surannées seraient une autre cause de retard de l'application des moyens suggérés par la Science, de même que la question financière, les dépenses étant actuellement à la charge des administrations des diverses localités, lesquelles disposent de ressources insuffisantes. Ledit auteur conclut enfin: $\therefore$ lorsque tous ces défauts d'organisation seront élininés, l'on peut garantir que la question des Sauterelles, qui pour plusieurs régions de la Russie est des plus graves, pourra être résolue d'une façon rapide et définitive. En l'état actuel des choses, il n'est possible d'obtenir que des résul- 
tats partiels et temporaires, encore que la technique russe possède des qualités incontestables et que l'on ait établi des procédés de lutte et des plans d'organisation élaborés d'une façon exceptionnellentent complète, en se basant sur une grande expérience ».

Tous les renseignements contenus dans les documents rassemblés à la suite de notre enquête ont été soigneusement coordonnés et distribués dans les divers chapitres dans lesquels est divisé le présent volume, dont le plan est celui généralement employé pour des travaux dont les éléments doivent se prêter à des études de nature comparative. Le travail de rédaction, qui a nécessité des soins minutieux et une grande précision pour coordonner, conformément au plan établi, les renseignements scientifiques et techniques contenus dans les divers documents, a été confié à M. le Prof. G. Trinchieri, rédacteur attaché à notre Section des Maladies des Plantes.

Les documents que nous publions montrent quelles sont, à l'heure actuelle, les connaissances acquises sur les mœurs et les agissements des insectes à l'étude et les moyens employés pour les détruire. Parmi ces documents, il y a lieu de comprendre la Bibliographie qui fait suite à l'exposé général et qui peut être considérée, croyons-nous, comme la documentation bibliographique des orthoptères nuisibles la plus complète, publiée après celle contenue dans l'ouvrage de M. KÜNCKEL, D'HERCULAIS.

Si, d'une part, il apparaît de notre Rapport que l'on ne possède pas encore, dans tous les cas et.avec la précision nécessaire, tous les éléments indispensables pour orienter la lutte vers la suppression radicale de l'ennemi, on apprendra, du moins, à quel point on en est arrivé dans la solution des différents problèmes essentiels: distribution géographique des diverses espèces nuisibles, localisation des "régions permanentes" et détermination des "lieux de ponte ", métamorphose dans ses rapports avec les moyens de lutte, direction des vols, régions "sub-permanente" et "temporaire", agents naturels et moyens artificiels de destruction.

Ces derniers sont nombreux, mais on n'aura pas à remarquer de grands progrès fondamentaux surị les procédés appliqués dès le début de la lutte rationnelle.

Les animaux acridiophages, domestiques ou non, sont toujours considérés comme des auxiliaires précieux.

Des études et des tentatives n'ont pas manqué pour essayer de 
cétruire les déprédateurs par la diffusion artificielie des maladies, cles parasites qui attaquent l'insecte; mais, quant à des résultats décisifs, on les attend encore.

L'idée de détruire l'enneni arant sa sortie des coques ou des grappes ovigères est partout à la base des sỵstèmes de lutte; dans plusieurs localités, on pratique le ramassage de ces vothèques moyen très cô̂teux et qui ne permet d'atténuer les éclosions que dans une mesure pratiquement trop faible - ou l'on s'attache à en favoriser la destruction par les animaux ou bien par des piocliages et des labours très superficiels, qui laissent les œufs à la merci des influences atmosphériques, ou encore en tassant le sol.

Quant aux différents moyens mécaniques, les syrstèmes consistant à opposer des barrages à l'invasion, dérivent tous du même principe découvert, dans la seconde moitié du XIX siècle, par l'italien IIATTEI, qui le premier eut l'idée d'arrêter les acridiens dans leur marche, en dressant devant eux des barrières pourrues d'une surface glissante qui les rendait infranchissables: ce sont là les appareils cypriotes universellement connus. La toile est toujours largement employée sous la forme de "melhafas" ou suivant d'autres dispositifs plus ou moins similaires; souvent, pour les barrages, elle est remplacée par des bandes de zinc. Çà et là, on s'attache à attirer les ennemis dans des trappes, ou bien à les ramasser par des collecteurs traînés à la surface du sol infesté. La destruction par écrasement est toujours en honneur. L'emploi d'appareils spéciaux par lesquels on a remplacé les anciennes torches a permis de se serrir de la flamme d'une façon plus efficace, en brûlant du pétrole ou un mélange de pétrole et de benzine.

Des progrès ont été faits dans le mode d'emploi des insecticides; le nombre des formules est grand, mais bien qu'au fond il ne s'agisse que de substances essayées pour la plupart depuis longtemps, on n'est pas sans incertitude lorsqu'on s'attache à choisir les plus appropriées; d'autant plus que les avis contradictoires ne manquent pas, même lorsqu'il s'agit de substances employées avec uni succès assuré. Tel est le cas des sels d'arsenic qui ont leurs détracteurs, en raison du danger auquel on expose les hommes et les animaux.

On a également en recours à la pyrotechnie aérienne en essayant des engins explosifs pour détruire, ou tout au moins disperser les nuées đes ailés, en les empêchant de s'abattre sur un endroit déterminé. On a aussi essayé des mélanges asphyxiants, mais sans résultats appréciables. M. Guénaux, du Iaboratoire d'Entomologie de l'Institut 
National Agronomique de Paris, préconise des fusées ou bombes spéciales que 1'on lancerait à 50 mètres de hauteur, qui produiraient, en éclatant, des détonations stridentes et donneraient une fumée intense ou même des dégagements de gaz délétères.

L'exposé d'ensemble que nous publions, examine l'état actuel de la question dans la plupart des pays, en tenant compte de toutes les espèces de Sauterelles y constatées; il contient, donc, tous les éléments positifs ou négatifs pouvant servir de base à l'ouverture d'une discussion fertile au sein d'une Conférence réunissant les personnalités compétentes des divers pays, d'où, il faut l'espérer, jailliront de nouvelles lumières au sujet de ce qu'il reste encore à faire pour se débarrasser d'un des plus redoutables ennemis séculaires de l'agriculture.

C'est pourquoi le Comité Permanent a accueilli avec le plus grand empressement la proposition transmise par M. MALET par l'intermédiaire du Délégué de la France et des Colonies françaises, Vice-Président de 1'Institut M. Lours-DoP, qui, dans la séance du 27 mai I9I6, a exposé toute la portée pratique d'une semblable initiative. A sa voix se sont unies celles de MM. le Marquis CapperLI, Délég ué de 1'Italie, Président de l'Institut, le Dr. PIÑEIRo Sorondo, Délégué de l'Argentine, le Dr. Rovira, Délégué del'Uruguay, M. E. LEAO, Délégué du Portugal, qui représentent des pays où l'on consacre à la question des Sauterelles toute l'attention et l'activité qu'elle mérite, de même que celle de S. E. M. ZabieLLO, Délégué de la Russie, un des pays où ont eu lieu les premiers signes du réveil qui conduisit à la nouvelle orientation dans les systèmes de lutte.

L'Institut International d'Agriculture n'en est pas à ses débuts en fait d'initiatives visant à la lutte contre les fléaux des cultures; aussi peut-on dire que l'initiative actuelle n'est qu'une suite naturelle de ses nombreuses discussions et démarches qui aboutirent à la Conférence Internationale de Phytopathologie, qui se tint à Rome du 24 février au 4 mars I9I4 et arrêta la "Convention Internationale du 4 mars I9I5", par laquelle (art. I) "les Etats contractants s'engagent à prendre les mesures législatives et administratives nécessaires, en vue d'assurer une action commune et efficace contre l'introduction et l'extension des ennemis des végétaux ».

La même Convention prévoit (art. 2) la création, dans chaque pays, d'un Service gouvernemental de Phytopathologie, ayant, entre autres attributions, celle de faire des études et des recherches scienti- 
fiques et techniques et d'organiser la surveillance efficace des cultutures.

Or, il appert de notre Rapport " LEE SERVICE DE PROTECTION DES PLANTES DANS LES DIVERS PAYS ", qui servit de base documentaire à la dite Conférence, que divers pays possèdent déjà un Service bien organisé, beaucoup d'autres sont en voie de le perfectionner à l'exemple des premiers, d'autres encore se proposent de le créer. D'autre part, le chapitre du présent Rapport consacré à l'organisation de la lutte contre les Sauterelles, montre que dans tous les pays intéressés, 1'administration locale consacre à la question une grande partie de son activité ; presque partout, par conséquent, on est en mesure, sans besoin d'un effort exceptionnel supérieur aux possibilités actuelles, de suivre tel plan rationnel d'investigations que sauront tracer des personnalités compétentes.

D'ailleurs, l'idée de l'application du principe de la "vis unita " par un groupement opportun des pays situés dans des zones sujettes aux orthoptères déprédateurs, a aussi des précédents dignes de la plus grande attention et pouvant apporter une contribution précieuse aux travaux de la Conférence préconisée.

Dans les pages qui suivent, l'attention du lecteur sera certainement retenue par les passages consacrés au "South African Central Locust Bureau " établi à Prétoria en Igo6 et à la Convention internationale de "Défensa Agricola " relative à l'investigation des foyers initiaux du Schistocerca paranensis, signée à Montevideo le Io mai IgI3.

L'organisation africaine, établie à la suite d'une Conférence entre la Colonie du Cap, le Natal, le Transvaal, la Colonie du fleuve Orange, le Bassoutoland, fournit des indications précieuses relativement aux résultats de l'emploi d'un système de surveillance et d'avertissement du mouvement des insectes au moyen de cartes spéciales, de même qu'au sujet des résultats des moyens de lutte employés, résultats qui ont été des plus encourageants.

Quant à la Convention américaine, établie à la suite de la Conférence internationale de "Défensa Agricola " entre l'Argentine, la Bolivie, le Brésil, le Paraguay, et l'Uruguay, elle prévoit une série de mesures techniques et administratives qui fournissent de très importants éléments de discussion.

La décision du Comité Permanent concernant la nouvelle initiative de l'Institut, est libellée dans les termes suivants:

"Le Comité Permanent, saisi d'une proposition formulée par M. 
I_OUIS-Dop au non du Gouvernement du Maroc en vue de réunir à Rome, en conformité de l'art. 9 lettre $f$, de la Convention du 7 juin I905: une Conférence Internationale pour la lutte contre les Sauterelles, décide :

(I) d'adopter le principe de la proposition présentée par M. Lours-Dop au nom du Gouvernement du Maroc;

(2) d'élargir cette proposition en invitant tous les Etats adhérents à l'Institut à participer aux travaux de cette Conférence et à s'y faire représenter, s'ils le jugent utile;

"3) de réunir cette Conférence internationale avant la prochaine Assemblée Générale.

“4) Le choix de la date de la Conférence sera déterminé ultérieurement après les démarches que l'Institut est invité à faire dès maintenant auprès des Gouvernements intéressés ».

L'Institut ouvre les démarches prévues à l'alinéa 4 de ces décisions, par le présent Rapport, auquel sera donnée la plus large diffusion dans les milieux scientifiques et administratifs des pays sujets aux déprédations.

Dans les réponses à notre questionnaire, presque tous les Etats ont reconnu l'utilité d'une entente internationale comme moyen rapide d'arriver à une solution complète du problème, il y a donc lieu de croire que les mêmes critères sont à la base des désidérata de tous les intéressés. Partant, les nombreux renseignements de nature comparative que nous publions, permettront d'étudier d'ores et déjà le plan de travail le plus approprié que la Conférence envisagée aura à se poser.

Au moment d'établir ce plan, la question, il nous semble, se posera fondamentalement ainsi qu'il suit :

a) Dégager de l'ensemble de la documentation les connaissances définitivement acquises sur la base des questions "déjà résolues".

b) Déterminer quels sont les points encore obscurs et proposer les moyens de les éclaircir.

c) Proposer un programme d'organisation de la lutte sur la base des moyens reconnus les plus efficaces.

C'est la lumière qui peut jaillir de l'application du grand principe de la "coopération intellectuelle» que la Conférence internationale sera appelée à jeter sur l'important problème.

Certes, le mot "international» entendu dans son acception la plus large, paraîtra exagéré au moment d'établir les mesures à prendre 
pour détruire l'ennemi, d'autant plus que, les espèces nuisibles et les

- conditions locales n'étant pas partout les mêmes, ces mesures ne peuvent pas être partout entièrement identiques. Sur ce point, il sera peut être plus approprié de parler de mesures "interrégionales", s'étendant aux pays soumis, par leur nature géographique et orographique, aux déprédations des mêmes espèces.

Le mot nous paraît, parcuntre, très approprié quand on l'applique à la Conférence préconisée, puisqu’il est de la plus haute utilité que la question soit discutée par les personnalités autorisées de tous les paỹs, chacune d'elles étant susceptible, non seulement de porter la contribution de ses lumières pour la solution des différentes questions techniques, mais encore de suggérer des conseils quant aux groupements appropriés des Etats ayant intérêt à travailler de conserve.

Tels sont, succinctement, l'objet de ce Rapport, les matières : développées et les critères suivis pour son élaboration.

Rome, nozembre IgI6.

DR. J. MI. SACIATER

ff. Chet du Bureau des Renseignencribs agricoic. et des Malnities des Flantes. 


\title{
LA LUTTE CONTRE LES SAUTERELLES DANS LES DIVERS PAYS
}

\author{
I \\ HISTORIQUE E'T DISTRIBUTION GÉOGRAPHIQUE \\ DES SAUTERELLES
}

Parmi les ennemis en nombre toujours croissant qui ravagent ou menacent sans trêve l'agriculture, il n'en est peut-être que quelquesuns qui soient connus depuis une époque aussi reculée, qui soient aussi universellement répandus et qui occasionnent aussi fréquemment des dommages considérables, que les insectes désignés communément sous le nom générique de sauterelles (I).

Mentionnés déjà dans de très anciennes compositions poétiques de l'Inde; décrits à plusieurs reprises dans la Bible, mais en particulier et de la manière la plus expressive comme constituant la huitième plaie d’Egypte; représentés dans les sculptures de Ninive et de Babylone ; connus des premiers écrivains, naturalistes et historiens grecs, romains et arabes, ces orthoptères ont été, à toutes les époques et dans toutes les parties du globe, et sont encore actuellement, un objet de vive curiosité pour le profane,

(I) A rrai dire, le nom de sauterelles devrait être réservé, d'après la majorité des auteurs, aux orthoptères sauteurs de la famille des Phasgonurida, désignés souvent à tort, selon W. F. KIRBY, sous le nom de Locustida (locustides) ; tandis que les orthoptères également sauteurs, mais pourvus aussi d'autres caractères importants qui les distinguent nettement des premiers, et beaucoup plus redoutables relativement à ces derniers, à savoir les insectes qui - au dire du savant précité - rentrent dans la famille des vrais Locustida, souvent dénommés Acridiida (acrides, acridides, ou acridiens), devraient porter le nom de criquets. Néanmoins, dans certaines régions francaises, y compris l'Afrique du Nord, les deux noms vulgaires de sauterelle et de criquet s'appliquent l'un et l'autre aux vrais Locustida, et l'on désigne plus spécialement par le premier de ces noms l'insecte pariait ou adulte muni d'ailes; le second sert plutôt à indiquer l'jinsecte à l'état larvaire, c'est-à-dire quand il est encore dépourvu d'ailes et qu' il est en train de subir ses différentes mues.

Quoi qu'il en soit, comme, en dehors du stade évolutif de l'insecte, l'usage a prévalu dans le langage commun d'étendre aussi la dénomination de sauterelles aux acridides, nous avons cru, afin de ne pas créer de confusion dans l'esprit du lecteur, devoir nous en tenir à la terminologie la plus généralement adoptée. D'autre part, il faut considérer aussi que dans les bandes et dans les dévastations, quelques représentants des sauterelles proprement dites (locustides) se mêlent parfois aux acridides. 
une source de préoccupations pour l'agriculteur, une matière importante de recherches et d'études pour l'entomologiste.

'Toutefois, s'il est possible, en remontant aux époques les plus reculées de l'histoire, de trouver fréquemment le souvenir plus ou moins précis des sauterelles et de leurs ravages, il n'en est pas toujours ainsi lorsqu'il s'agit d'attribuer avec une certaine approximation, sinon avec une sûrété absolue, une date aux premières apparitions de ces redoutables insectes dans chacune des différentes parties du globe.

Ce chapitre est consacré à tous les renseignements que nous avons pu réunir au sujet de 1'histoire et de l'existence des sauterelles sur toute la surface de la terre; nous tâcherons de suivre, autant que possible, dans l'énumération des pays qui seront l'objet de notre examen, 1'ordre géographique.

EUROPE. - On n'a pas souvenir de dommages causés par les sauterelles en $\mathrm{N}$ orvège, en $\mathrm{D}$ a n e $\mathrm{mark}$, en $\mathrm{Ir} 1 \mathrm{ande} \mathrm{ni} \mathrm{dans} \mathrm{la}$ Principauté de Monaco.

De plus, bien que divers auteurs - par exemple, E. Browx (IS70-7I)

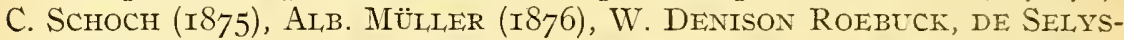
Longchamps (I877), Ad. Targioni 'TOZze'Tti (I882), J. KÜNChEL D'HeRCulais (I893-I905), L. REH (I9I3), Chas.-P. LounsbuRI (I9I5) - aient fait mention d'apparitions de sauterelles signalées en G r a n d e - B r e t a gne, aux Pays-Bas, en Belgique, en Su is se, aux environs de G i b r a 1 t a $r$, etc., il semble toutefois, d'après les rapports officiels parvenus à 1'Institut international d'Agriculture, que la présence de ces insectes n'ait pas donné lieu à de graves plaintes dans les pařs susmentionnés.

En A 11 e m a g n e, l'importance économique des sauterelles a été de tout temps très limitée. L'agriculture allemande n'a jamais eu, en effet, à déplorer de véritables ravages du fait de ces insectes.

Ajoutons que les orthoptères des deux familles prenuent des noms vulgaires différents selon les diverses langues. En allemand, par exemple, on les désigne, d'une manière générale, sous le nom de "Heuschrecken 》 et l'on dénomme plus particulièrement "Feldheuschrecken ", "Wanderheuschrecken " les représentants des vrais Locustida et "Laubheuschrecken " les espèces de Phasgonurida; en anglais, les noms de "grasshoppers " et de "locusts", bien que souvent appliqués indistinctement aux mêmes insectes, servent aussi, l'un à désigner les Phasgonurida, l'autre les vrais Locustida; on dénomme toutefois arec plus d'exactitude les espèces de la première famille "long-horned grasshoppers" et celles de la seconde "short-horned grasshoppers", "true locusts » et "migratory locusts"; en espagnol, en italien, en portugais, et en roumain on comprend le plus souvent les Phasgonurida et les rrais 'Locustida sous un nom commun qui est respectivement de "langosta ", "cavallette ", "gafanhotos " et " lăcustǎ ".

En hollandais, en outre du nom général "sprinkhanen", on emploie celui de "sabelsprinkhanen " pour indiquer plus spécialement les Phasgonurida et celıi de "veldsprinkhanen " pour désigner les vrais Locustida.

En chinois les sauterelles sont appelées "wan ", "wanson ", " naï " et $\in$ n japonais " inago ", «batta ». 
Quoi qu'il en soit, d'après les documents de l'abbaye de Fulda, ils firent leur apparition en Allemagne dès 1'année 873 ; on possède d'autres données à ce sujet pour les années I333, I338, I475, I527, I542, I636, I686, I693, I696, I7I2, I7I4, I7I5, I7I9, I727, I728, I729, I730, I73I, I734, I746, I747-50, I752-54, I759-63, I803, I825-27, I 856 , I859, I873, I875, I876, I877, I887, I889.

Il convient d'ajouter, il est vrai, que malheureusement la véracité d'une bonne partie de ces renseignements n'est rien moins que certaine. Ils sont pris le plus souvent, surtout les moins récents, dans de vieilles chroniques, que la tendance à raconter des événements extraordinaires et merveilleux peut avoir entraîné à des exagérations ou à des faussetés, même lorsqu'il s'est agi de nous transmettre des faits relatifs au fléau des santerelles. Quoi qu'il en soit, même dans les tout premiers temps, les invasions de sauterelles doivent avoir appartenu, en Allemagne, à la catégorie des grandes raretés, car OKEN (I836) a écrit que les plus vieux paysans, y compris cenx de l'Allemagne orientale, se souviennent à grand'peine d'une apparition de ces insectes. On n'a pas d'ailleurs de données sur l'étendue du territoire qu'ils ont visité aux différentes époques.

En Serbie, la première apparition de sauterelles, en masse, a été notée, par F. PANCic, en I849, dans les champs du village de Korman (dép. de Kragujevac). Le vol qui s'y était abattu, chassé par les paysans, s'éleva pour descendre de nouvean dans les villages voisins où il produisit, surtout à Badgnevac, des dégâts considérables.

Plus tard, il y eut une apparition en masse au mois de mai Igo6, entre les villages de Vinci et de Požezena (dép. de Požarevac).

On peut ajouter que, jusqu'en IgI2, les sauterelles n'ont pas constitué une menace vraiment sérieuse pour l'agriculture serbe.

I1 faut remonter jusqu'en I850, pour trouver trace des sauterelles dans l'île de M a $1 \mathrm{t}$ e; cette année-là, elles apparurent sur la côte méridionale et occasionnèrent de grands dommages à Zabbar et à Vied-elGhain.

A part ce premier et unique souvenir, la tradition populaire vent que la procession religieuse de Saint-Grégoire, qui se rend tous les ans de Casal Paula à Zeitun, soit précisément l'accomplissement d'un vou fait dans l'intention de préserver l'ile de ce fléau. Jusqu'en IgI2 on n'a dû déplorer aucune dévastation.

En F r a n c e, le souvenir des sauterelles date déjà de 1'année 874 . JULIUS OBSEQUENS écrit en effet que cette année-1à "le vaste et fertile pays de France ayant été envahi par d'innombrables sauterelles, celles-ci finirent toutes par aller se noyer dans la mer britannique d'où, ayant été rejetées ensuite sur le rivage de France, elles produisirent, par leur mauvaise odeur, une mortalité notable dans ce royaume ". Au dire d'Urisse ArdoroVANDI, il en fut de même en I542 en France (et en même temps en Allemagne et en Pologne) où ces insectes "occasionnèrent d'abord la famine, puis la peste, née de la puanteur qui s'exhalait de leurs corps morts, fit périr une énorme quantité de personnes ».

Les sauterelles ont été plusieurs fois signalées dans le Midi à des époques 
successives; par exemple, en I6r3 (Marseille, Arles), en I824 (SaintesMaries, Arles), en I832-33.

Elles ont été également observées plus tard sur le territoire français, entre autres années, en I857, I868-70, I874-76, I887 (dans le Sud-Ouest).

Durant les années I8g8-Igor les sauterelles se sont propagées dans les cultures du Sud et au mois de juin rgor, en particulier, la présence de ces insectes prit des proportions menaçantes (Camargue, cultures au sud d'Arles). La seconde quinzaine de juillet de la même année, des vols de sauterelles furent signalés aussi dans le Sud-Ouest et dans quelques départements du Centre, jusqu'alors indemnes. Les Charentes, déjà fortement éprouvées en Igor, le furent encore l'année suivante. Des sauterelles ont été également signalées en France, en I907. A la fin de juillet Igog, eut lieu près de Chambéry (dép. de la Savoie) une soudaine apparition de ces insectes. On les a signalés aussi en IgI4 dans le Gard et les Bouches-du-Rhône (Camargue).

Bien que des données précises manquent à cet égard, il est permis de supposer qu'en $\mathrm{P}$ o r t u g a 1, l'existence des sauterelles remonte à une époque assez ancienne. On peut ajouter que ces orthoptères représentent une des plaies dont l'agriculture du sud du pays a plus ou moins périodiquement souffert, surtout dans les provinces importantes d'Alentejo et d'Algarve, dans les régions agricoles qui bordent les rives du Guadiana.

Ces insectes furent signalés en Portugal pendant l'été de I 876 .

Quant aux années plus rapprochées, il y eut au commencement du mois de juin I898, à Castro Marim, une grande apparition de sauterelles, qui resta gravée dans la mémoire des populations de la région, car la bande, qui avait un front de $3 \mathrm{~km}$, employa de 6 à $\delta$ jours pour traverser la zone agricole de Castro Marim; elle se répandit ensuite dans les territoires de Vila Rial de S. Antonio et de Tavira.

En I 899 les territoires particulièrement ravagés furent ceux de Tavira, Vila Rial de S. Antonio, Castro Marim et Alcoutim, dans le district de Faro; la même année, les sauterelles causèrent des dommages aussi dans quelques territoires du district de Portalegre, et principalement dans le territoire d'Elvas, dans les domaines situés entre les fleuves Guadiana et Caia; et au mois de juin de la même année, elles se montrèrent aussi en grande quantité dans le territoire de Mertola, district de Beja.

Pour se faire une idée de l'intensité que prit le fléau en IS99, il suffira de dire que l'on ramassa $559559 \mathrm{~kg}$ de sauterelles (chaque kilogramme comprend en movenne I5 000 insectes) et que les œufs pondus cette année-là se trouvaient en de telles quantités, daus les districts de Faro et de Beja, que l'on reconnut des millions de mètres carrés de superficie infestée de plus de 2000 coques ovigères ou oothèques par mètre carré, chaque oothèque contenant en moyenne 30 œufs!

En I goI, les sauterelles ont encore été le fléau de l'agriculture portugaise; on reconnut leur présence dans les districts de Castelo Branco, Beja, Faro, Lisbonne, Vise11, Portalegre, Leiria, Coïmbre et Evora ; les attaques les plus sensibles se produisirent sur quelques parties des territoires de Proença-a-Nova, Monsanto, Pedrogam, Esscalas de Baixo, Vila Telha de Ro- 
dam, Castro Verde, Aljustrel, Mertola, Casevel, Messejana, Ourique, A1coutim, Castro Marim, Tavira, Loulé, Campo Maior, Niza, Ellvas, Santa Eulalia et Marvão.

Il y eut, cette année-là, une des invasions les plus nombreuses; elle toucha les zones agricoles importantes des provinces de Beira Baixa, Estrémadure, Alentejo et Algarve.

Une bande de $8 \mathrm{~km}$ de long sur 6 de large apparut le 20 juin IgoI dans le district de Portalegre. Dans ce même district la superficie infestée cette année-là par les sauterelles fut de 7600 ha. Pendant la campagne de Ig0I on ramassa I $136896 \mathrm{~kg}$ d'insectes.

En I902, I903, I907 et I909, de nouveaux ravages furent signalés dans le district de Portalegre et spécialement dans les territoires d'Elvas, Campo Maior et Arronches; la plus grave de ces invasions fut celle de 1907 oì la bande occupait une étendue de 6 à $7 \mathrm{~km}$.

En I9I4, les sauterelles firent leur apparition dans le district de Santarem (domaines de Agolada et de Fajarda); les mesures prises avec une grande rapidité ont permis de localiser le foyer d'infection.

En I9I6, le fléau a envahi les territoires de Niza, Gavião, Crato, Ponte de Sôr, Abrantes.

Ces orthoptères firent leur première apparition en $\mathrm{E} s \mathrm{p}$ a g $\mathrm{n}$ e dès l'année I584; on les vit à Villanueva de los Infantes et dans Campo de Montiel.

De nombreux auteurs expriment l'avis que les sauterelles s'étaient déjà présentées antérieurement à cette époque dans la vallée d'Alcudia (Ciudad Real) ; mais cette opinion ne paraît pas avoir été confirmée.

Quoi qu'il en soit, elles sont devenues avec le temps un des pires fléaux qui aient ravagé l'agriculture espagnole.

Afin de démontrer que l'importance des sauterelles, comme agents de dommages parfois très considérables pour le pays, ri échappait pas à la sollicitude des rois d'Esspagne, on cite, entre autres, le fait qu'en I619, Philippe III transféra le juge D Francisco de Salvatierra du tribunal de Grenade à Alcázar de San Juan, en lui faisant remettre immédiatement une première somme de 50000 ducats ( $50000 \mathrm{fr}$ environ), destinée à pourvoir aux frais nécessaires pour commencer la lutte contre ces insectes.

Dans les années $1685-87$, les sauterelles visitèrent la province de Lérida; en I755, I779, I80I, I824, I84I, I849 et I855, elles firent leur apparition dans celles de Ciudad Rea1, Madrid et Tolède; le fléau n'ayant pas été combattu à temps, les dommages qu'il produisit quelques années après prirent une telle importance que les populations tombèrent dans la plus profonde misère.

En I 876, I 8 provinces furent infestées ; on cite parmi les plus atteintes celles de Valladolid, Palencia, Alméria, Jaen, Léon, Murcie, Cacérès et Madrid.

Passant à des années encore plus récentes, une apparition fut signalée en $x 899$; pendant la campagne de lutte I900-0I, on s'assura que dans tout le territoire continental espagnol, il y avait une superficie infestée de plus de $214^{000}$ ha, répartie en proportion plus ou moins grande parmi les pro- 
vinces suivantes, énumérées par ordre d'importance de l'infestation: $\mathrm{Ba}$ dajóz, Cacérès, Cordoue, Ciudad Real, Jaen, Séville, Salamanque, Huelva, Alméria, Madrid, Gérona, Léon, Tolède, Cuença, Grenade, Avila, Saragosse, Palencia, Zamora, Murcie, Valladolid et Malaga.

Plus récemment encore, pendant la campagne de IgIo-II, une étendue de territoire très considérable fut également reconnue infestée; toutefois, tandis que contrairement à ce qui s'était produit en I900-0I, le lléau laissait intactes les provinces d'Avila, Gérona, Grenade, Murcie, Palencia, Valladolid, Zamora et Saragosse, les sauterelles furent signalées dans deux autres provinces, celles d'Albacète et de Cadix.

L'étendue du territoire infesté, constatée pendant la campagne IgI I-I2, fut également très grande; à la liste des provinces déjà atteintes en IgIo-II il faut ajouter celle d'Avila.

Pour citer des faits très récents, on a constaté pendant la campagne I9I3-I4 que, dans tout le territoire continental, la superficie infestée atteignait plus de 28000 ha. Les provinces les plus éprouvées - les mêmes que celles de la campagne précédente - furent les suivantes par ordre d'importance : Badajóz, Cacérès, Séville, Léon, Tolède, Alméria, Cordoue, Cadix, Madrid, Ciudad Real, Huelva, Albacète, Malaga, Jaen, Salamanque, Avila et Cuença. De nouvelles apparitions ont été signalées en Espagne (Andalousie, Estrémadure, Ia Manche, etc.) dans ces derniers temps.

En ce qui concerne 1' I t a $1 \mathrm{i}$ e, les écrits des plus anciens auteurs, parmi lesquels Pline L'Ancien, Trte-Live, Julius Obsequens et Paul. DIACRE, font déjà mention des sauterelles.

On peut dire qu'à toutes les époques ces insectes ont fourni à un bon nombre de chroniqueurs, d'historiens et de chercheurs une matière qu'ils ont traitée fréquemment et parfois même plutôt largement, s'occupant tour à tour des différentes parties de l'Italie.

A une époque plus rapprochée et aujourd'hui encore la question des sauterelles est pour l'Italie un sujet de grave préoccupation.

La confirmation la plus claire en est l'importance des sommes affectées à la lutte contre le fléau. Nous citerons, à titre d'exemple, le fait qu'en IS69 le Gouvernement italien ouvrit, à cet effet, un crédit de 300000 lires, et la Sardaigne employa à elle seule dans les opérations de destruction plus de 500000 lires. Le roi Victor-Emmanuel II, et avec lui les princes de la Maison de Savoie, subventionnèrent largement de leur cassette particulière les communes qui eurent à souffrir des sauterelles. Le résultat de cette campagne de lutte fut la destruction de $105000 \mathrm{hl}$ d'insectes, pesant I I7I $400 \mathrm{~kg}$.

Pour citer un exemple plus récent, on employa en rgog-Io, dans la lutte contre les sauterelles, une somme de 200000 lires, votée comme fonds extraordinaires par le Parlement, en vue de la gravité exceptionnelle présentée par le fléau dans de nombreuses parties de 1'Italie méridionale.

Dans les budgets du Ministère de l'Agriculture pour les années financières IgIO-II, IgI3-I4 et IgI5-I6 on a inscrit respectivement les sommes de 250000 , I60 o00 et I00 000 lires, pour les mesures contre les sauterelles.

Des apparitions extraordinaires de ces insectes furent signalées jadis en Lombardie: déjà, paraît-il, en 873 (territoires de Milan, Crema, Lodi, 
Brescia), et plus tard en I363-65 et I389 (territoire de Milan), en I54243, I647, I728 (territoire de Mantoue), en I845 (prov. de Milan), en I862 (prov. de Sondrio), etc.

En I 478, il y eut en Vénétie une apparition notable de sauterelles, qui fut suivi de disette. D'autres invasions encore furent constatées, dans les provinces vénitiennes, en $\mathrm{I}_{542-43}$ et en $\mathrm{I} 647$; on en a un souvenir certain pour la province de Vérone en $1874-75$; pour la même province et aussi pour celle de Trévise en I 882 .

L'Emilie eut également à déplorer des dommages causés par les sauterelles: en 873 (dans le territoire de Plaisance), en I232 (dans ceux de Bologne et de Mirandola: Sigonro, entre autres auteurs, en a traité), en I277 (des chroniqueurs et 1'historien GHIRARDACCr en parlent), en I299, I363-65 et I369 (en Romagne et dans le territoire de Plaisance), en I728 (dans les territoires de Ferrare et de Mirandola), en I747 (dans le territoire de Mirandola), en I824 (dans le duché de Modène), en I825 (dans le territoire de Mirandola), etc.

Quant à la Toscane, citons parmi les apparitions les moins récentes celles de $1573-74$ et de 1647 (dans la maremme siennoise); puis les ravages de I7II-I6 (dans les provinces de Pise et de Sienne, dont deux anonymes, que 1'on croit être GIUSEPPE DEL PAPA et TOMASO BUONAVENTURI, traitèrent largement dans des rapports séparés), de I806, de I 839 (maremme siennoise), de $1879-80$ (prov. de Sienne: San Quirico d'Orcia, Castiglion d'Orcia, Pienza), de $I 88 I-82$ (encore dans la prov. de Sienne), et, plus récemment, les ravages de I892-93, à Brozzi, province de Florence.

Des apparitions de sauterelles eurent lieu dans les Marches, entre autres durant les années I363-65, I389, I876 (prov. de Pesaro).

Plusieurs apparitions furent enregistrées dans la Campagne romaine, en I556, I656, I7I7, I807-I5 (d'abord le territoire de Marino et d'A1bano, puis toute la Campagne: DoRIA en a traité), en I $82 \mathrm{I}, \mathrm{I} 825$, et, plus récemment, en I888 et en I894. Chieti).

Les sauterelles furent signalées dans les Abruzzes en I876 (prov. de

Aucun document historique ne rapporte de grandes apparitions dans le Molise; par contre, on y a constaté de temps en temps la présence de ces orthoptères dans une mesure limitée tant pour la superficie du territoire occupé que pour la durée de l'occupation.

On peut évaluer à $350 \mathrm{~km}^{2}$ environ la superficie du territoire récemment visité (IgII-I2) à la limite de la province de Foggia, d'où les sauterelles se sont répandues dans les communes de Campomarino, Portocannone, San Martino in Pensilis, Ururi, Rotello, Santa Croce di Magliano, San Giuliano di Puglia et Colletorto.

Pour la Campanie on conserve le souvenir douteux d'une apparition qui aurait eu lieu déjà en 873 ; des données plus certaines au sujet de la présence des sauterelles dans la même région remontent aux années 1765 , I868-70 (prov. de Naples; la dernière de ces années, la prov. de Salerne fut également visitée) et I877-78 (prov. de Caserte). En I897, ces orthoptères attirèrent encore l'attention dans la province de Caserte où des dommages notables ne turent plus signalés jusqu'en I9ro ; à partir de cette époque, les sauterelles 
réapparurent jusqu'en I9I2. La zone infestée comprit en grande partie le territoire de Cellole, commune de Sessa Aurunca, sur plusieurs kilomètres d'étendue jusqu'à la mer.

Dans la Pouille - outre les apparitions de 58I av. J.-C., de I23I, I249, I363-65, I389- celles de I54I-42 et de I56I restèrent tristement célèbies. En I 662 , les sat1terelles causèrent des dommages tellement graves, que le Gouvernement se trouva dans la nécessité non seulement d'exonérer les fermiers des terıains domaniaux de leurs charges de l'année, mais de réduire aussi de moitié celles de l'année suivante.

D'autres apparitions eurent lien en I722, I727, I809, I8I3, IS5I (année où les santerelles ravagèrent les campagnes de la Capitanate ou prov. de Foggia); en I869-70; en I877-78 (prov. de Foggia : territoires de San Giovanni Rotondo et de Manfredonia; prov. de 'Terre d'Otrante ou Lecce) ; en I88I (prov. de Bari).

Des apparitions ont été encore récemment signalées dans la province de Lecce. Le territoire que les sauterelles y ont visité est très étendu, car il comprend toute la zone qui de Palagiano et Palagianello s'étend jusqu'à Mottola et Castellaneta, la zone de Manduria à Grottaglie et la région très vaste des territoires de Nardò, Leverano, Veglie et Salice.

Les sauterelles ont fait également leur apparition, en quantités notables, dans la Basilicate (prov. de Potenza) dès une époque très reculée (I249); leur présence y a été constatée aussi en I864, I869-70, I877-78 (commune d'Aliano), en I882 et au printemps de IgIo.

Le premier souvenir des apparitions de sauterelles en Calabre remonte à I I83 (dans la vallée du Crati); il y en eut en I249, en I876 (prov. de Catanzaro: Monteleone), en I879-80 (prov. de Cosenza), en I882, IgoS-II (prov. de Catanzaro: communes d'Isola Caporizzuto, de Cutro, Strongoli, Rocca di Neto, Cirò, Cropani, Roccabernarda, Casabona, Soverato, San Sostene, Staletti, Squillace, etc.); en Igog-Io (prov. de Reggio) ; en I9I2 (prov. de Catanzaro: Isola Caporizzuto, Cotrone, Cutro, Pizzo, Nicotera, San Sostene).

La superficie infestée a toujours été très étendue, mais il est difficile d'en établir avec précision la mesure.

Une des régions italiennes qui, dans la suite des temps, a été fréquemment visitée et qui a souffert de très grands dommages, est, sans doute, la Sicile.

Des apparitions notables y furent déjà constatées pendant la période arabe $(842)$, dans la province de Girgenti. Après un très long intervalle pendant lequel il est présumable que ce ne sont pas les sauterelles qui firent défaut, mais plutôt le soin d'en enregistrer la présence, on retrouve le sourenir de ces orthoptères en I355 et I363 lorsqu'ils occupèrent de grandes étendues dans presque toute l'île. Ensuite, le témoignage de J. J. ADria, confié à un manuscrit du XVI ${ }^{\mathrm{e}}$ siècle ( $\mathrm{I}_{540}$ ), conservé - comme le rappelle T. DE STEFANI PEREZ - à la Bibliothèque communale de Palerme, nous apprend qu'en I 490 , les sauterelles venant de l'Afrique et réunies en nuées immenses et d'une épaisseur considérable, au point d'intercepter les rayons du soleil, s'abattirent sur la Sicile, dans le territoire de Mazzara, et, le long du littoral, en touchant, entre autres localités, Sciacca, Girgenti, Gela (aujour- 
d'hui Terranova), Camerina (Santa Croce Camerina), elles atteignirent Syracuse, en semant la ruine ; elles détruisirent les moissons, les vignes, toutes sortes de fruits et elles n'épargnèrent même pas l'écorce des arbres qui en restèrent dépouillés pendant trois ans.

Files apparurent encore dans 1'île - des observations antérieures font détaut, à ce qu'il semble -- en I637 (campagne de Palerme); I655 (dans plusieurs localités non précisées) ; I656-57 єt I659 (dans tòute 1'île); I687 (prov. de Caltanissetta); I688 (dans toute 1'île); I699 (dans plusieurs provinces, mais surtout dans celle de Syracuse); I703-04 (prov. de Caltanissetta); I708-I2 (dans toute 1'1̂le); I755-56 (dans plusienrs localités non précisées); I784, I789 et I796 (dans toute l'île) ; I80I-I8I3 (dans plusieurs provinces); I829 (prov. de Catane); I832-33 (prov. de Caltanissetta); I839 (dans plusieurs parties de 1'1̂le); I856-59 (dans la région des Madonie); I863 (prov. de Messine); I865 (prov. de Trapani); I866 (toute l'île de Pantelleria); I869-70 (dans plusieurs provinces de 1'île); I $877-78$ (prov. de Trapani, dans la campagne de Castelvetrano); I 882 (prov. de Catane et de Caltanissetta); I883 (prov. de Caltanissetta: campagnes d'Aidone); Ig06-I2 (prov. de Palerme: Petralia Sottana, Petralia Soprana, Geraci Siculo); Igog-Io (prov. de Messine et, plus exactement, en Igog, dans les communes de Tripi et Furnari, dans celle de Tusa, à Forza d'Agrò et à Locadi ; en .IgIo, surtout à Fiumedinisi, Roccalumera, Mandanici, S. Teresa Riva [Furci], Casalvecchio, Scaletta Zanclea, Messine [Briga, Castanea, etc.], Barcellona, Lipari, Librizzi, Motta d'Affermo); IgIo-II (prov. de Pale1me: Terrasini et Polizzi Generosa); I9ı-I2 (prov. de Trapani: Monte San Giuliano; prov. de Girgenti: Racalmuto, Cammarata, Grotte, S. Margherita et Montevago); I9II-I2 (prov. de Palerme: Gangi; prov. de Caltanissetta: Aidone, Piazza Armerina, Castrogiovanni, Campofranco, Resuttano et Sutera; prov. de Catane: Sperlinga et Cerami; prov. de Messine: Tusa et Tripi).

On estime, qu'en I9I2, la superficie infestée par les sauterelles atteignait, dans la province de Palerme, 3000 ha pour Petralia Sottana et Petralia Soprana, 4000 ha pour Gangi et 2500 ha pour Geraci Siculo; dans la province de Catane, Ioos ha pour Sperlinga ; dans la province de Caltanissetta, I200 ha pour Aidone, Castrogiovanni, Resuttano, Campofranco et Piazza Armerina ; dans la province de Girgenti, Iooo ha environ pour Racalmuto, Cammarata, Grotte, S. Margherita et Montevago; dans la province de Trapani, IOo ha; dans la province de Messine, 200 ha; en tout, I3 ooo ha.

Des infestations excessivement nombreuses et graves furent constatées aussi en I9I3, dans les territoires des communes de Bronte, Maletto, et Randazzo (prov. de Catane), sur une étendue de plus de 2000 ha ; et, en I9I4, sur une très vaste zone au nord et au nord-est de Randazzo et dans les communes de Santa Domenica Vittoria, Castell'Umberto, Ucria, Tortorici, Raccuja et Sinagra, dans la province de Messine.

Les infestations de la zone de 1'Etna et des collines et montagnes situées au nord de ce volcan, comprenaient, en IgI4, une superficie de plus de 30000 ha, et celles qui avaient déjà eu lieu dans la région des Madonie (Petralia Sottana, Petralia Soprana, Polizzi Generosa, etc.) plus de. 20000 ha. 
A propos de l'importance considérable de l'infestațion constatée dans les communes de Maletto et de Bronte, on raconte que les sauterelles s'étant abattues en grand nombre sur les rails dı chemin de fer, causèrent plusieurs fois 1'arrêt des trains dirigés vers Maletto, les roues des locomotives patinant sur la couche gluante formée par les insectes écrasés. relles.

La Sardaigne a été et est souvent sujette aux ravages des saute-

Dès les temps les plus reculés les autorités locales se préoccupèrent des dommages causés par ces orthoptères et cherchèrent les moyens de conjurer le fléau; il en est question dans certaines mesures prises par les anciens dominateurs de l'île et dans des "pregoni " des vice-rois de Sardaigne ( 1722 , I755).

Parmi les infestations générales les plus anciennes et les plus graves, citons celle de 1652 décrite par le père Grokgio AlEo. Des dommages très considérables furent constatés aussi en I864 (prov. de Cagliari : communes de Simaxis, Palmas Arborea, Sili). Il faut également signaler l'intensité d'une apparition qui eut lieu en I 868 et pendant laquelle les innombrables insectes formèrent durant plusieurs heures une sorte de manteau impénétrable recouvrant une bonne partie du sud de la Sardaigne; la province de Cagliari dépensa presque un demi-million de lires pour les combattre. D'autres apparitions se produisirent en I869-70, I877-78.

En I894, le Campidano d'Oristano (prov. de Cagliari), fut littéralement occupé; cette infestation qui se prolongea pendant plusieurs années de suite, bien qu'avec une intensité allant toujours en décroissant dut tirer son origine de foyers formés quelques années auparavant dans la zone située entre l'embouchure du Tirso et l'étang de Santa Giusta.

En Ig03, les sauterelles occupèrent une partie de la province de Sassari (depuis Terranova jusque vers Tempio), d'où elles passèrent en partie dans le Campo di Sant'Anna, territoire de Marrubiu (prov. de Cagliari) ; en IgIo, elles infestèrent une vaste étendue de terrains dont le centre était Santa Giusta et elles furent signalées dans des proportions encore plus grandes en I907-08 dans la province de Cagliari.

Ein I909, elles occasionnèrent de grands dommages dans 32 communes de la province de Cagliari, parmi lesquelles Bosa, Capoterra, Collinas, Fluminimaggiore, Gonnosfanadiga, Guspini, Iglesias, Marrubiu, Meana Sardo, Mogoro, Morgongiori, Oristano, Pabillonis, Palmas Arborea, Santa Giusta, San Vero Milis, San Vito, San Gavino, Samugheo, Sardara, Serbariu, Serrenti, Sili, Simaxis, Sorradile, Tortoli, Villacidro, Villamassargia, etc.; l'étendue du territoire infesté fut d'environ 34000 ha.

En I9Io, l'infestation s'étendit à I24 communes de l'île - parmi lequelles Bannari d'Usellus, Collinas, Decimomannu, Gonnostramatza, Furtei, Iunamatrona, Samassi, San Gavino, Sanluri, Sedilo, Serrenti, Siddi, Simaxis, Santulussurgiu, Sarroch, Sardara, San Pietro Pula, Terralba, Uras, Villanova Forru, etc. dans la province de Cagliari - sur une superficie totale de I92 993 ha; dans la commune de San Gavino seule, il y eut environ 200 ha infestés.

En I9II, une apparition de sauterelles eut lieu de nouveau dans 97 
communes déjà infestées l'année précédente et elle s'étendit à 28 autres ; les sauterelles envahirent une superficie totale de 53960 ha.

En I9I2, elles furent de nouveau signalées dans plusieurs communes de l'île, mais l'importance des dommages produits fut relativement assez faible en comparaison de ceux des années précédentes.

Dc nouvelles apparitions de ces insectes en Sardaigne ont eu lieu aussi en igr6.

On peut ajouter au sujet de la superficie totale des territoires infestés en Italie, que, variable d'une année à l'autre, elle ne paraît pas avoir dépassé, dans l'ensemble, I00 000 ha dans ces cinquante dernières années.

En A u $\mathrm{tr}$ i che, la présence des sauterelles aurait déjà été constatée en $1336-38$ (en Moravie) ; on rappelle à ce propos qu'une bande qui y apparut aurait occupé une étendue de $53 \mathrm{~km}$ environ.

D'autres apparitions furent signalées en $\mathrm{I}_{542}$ (dans la Val Sugana), en I684, I693-94, I749 (Budveis, dans le voisinage des villages de Hlinz et Gutwasser), en I 852 (près de Gratz), en I 857 (Korneuburg; près de Gratz, les aunes furent complètement dépouillés de leurs feuilles sur une superficie de plus de $57 \mathrm{~km}$ ), en I862-64 (Wiener Wald en I862; Höllenstein, Heuberg, Mittenberg près de Mödling, en I862-64 ; près de Tüffer [domaine de Gairach] en I864); en I866, I880 (Istrie), en Ig05 (Dalmatie). Dans la Styrie méridionale, sur les deux versants de la vallée du Sann, entre Cilli et Tüffer, sur une superficie de $6 \mathrm{~km}$ sur 4 (rive droite du Sann, de la vallée de Retschitz jusque presque au-dessus de Tremmersfeld, et, rive gauche, de Jagoce jusqu'à Jesernik), où il est présumable que les sauterelles étaient déjà connues depuis une douzaine d'années, ces insectes ont fait leur apparition en quantités considérables pendant l'été de I907; à la même saison et la même année, elles ont produit des dégâts à Littai en Carniole. Au printemps et au commencement de l'été de IgII, des ravages furent constatés aux environs de Frauheim près de Marbourg dans la Styrie méridionale.

Dans le Carso gorizien les dommages ont été de plus en plus considérables depuis I907 (vallée du Vippacco, Cernizza, Oppacchiasella, Pliscovizza, Gabrovizza, Sesana); en I 908 , les sauterelles occupèrent une superficie d'au moins $400 \mathrm{~km}^{2}$ (40000 ha) de Sesana jusqu'à la Porte de Fer (Zelezna Vrata) et de San Daniele jusqu'à Oppacchiasella, avec un centre d'infestation dans les environs de Comen, Cobillaglava et Gabrovizza.

Les sauterelles ont été signalées encore en Igog dans le Carso gorizien et en Dalmatie (île de Pago), et en IgII-I2, elles firent une nouvelle apparition en Dalmatie (environs de Vrlika et de Sinj).

Quant à la Ho n g ri e, pendant les trois ou quatre derniers siècles, elle était connue comme le "pays des sauterelles"; en effet, elle est toujours citée dans les chroniques et dans d'autres documents comme la contrée d'où proviennent les hordes immenses qui s'abattent, à certaines périodes, sur toute 1'Europe centrale.

Déjà au Moyen Age, "Hungaria ", "Moldavia " et "Tartaria " étaient les pays que l'on désignait comme les foyers d'origine de ces sauterelles migratrices, bien qu'il n'y ait aucun doute, du moins pour la Hongrie, 
que celle-ci ait été aussi infestée à son tour par des bandes venant de l'étranger.

De terribles apparitions de sauterelles furent enregistrées, en Hongrie, en I542 et en I684. Pour citer une époque plus rapprochée, en I 858 , ces insectes pullulaient dans le Hanság, au nord-est du lac de Fertö, entre les communes de Pomogy et de Bánfalva; en I888, ils furent signalés dans la commune de Péczel (comitat de Pest-Pilis-Solt-Kiskún); en I880-I89o, ils se multiplièrent dans les régions du nord-est du pays, dans le comitat de Szatmár, aux environs des communes de Parasznya et de Vitka, non loin du marais d'Ecsed.

L'apparition de Péczel, mentionnée plus haut (I888), n'était pas très considérable, mais elle eut des conséquences très graves les années suivantes, jusqu'en I893, le long des rives de la Tisza; en effet, 1'infestation ne s'étendit pas seulement au comitat de Pest-Pilis-Solt-Kiskún, mais elle gagna les comitats de Heves, Jásznagykúnszolnok, Csongrád, Békés, Torontál et les environ des communes de Szeged et de Hódmezövásárhely. Les santere1les se trouvaient en très grandes quantités surtout dans lt comitat de Torontál (entre 'Tiszaszentmiklós, Nagykikinda et 'Törökbecsa), à Szeged et dans le comitat de Jásznagykúnszolnok (Puszta-Eicseg).

Depuis cette époque, ces insectes ont épargné la Hongrie jusqu’en Igo4, année où ils firent une nouvelle apparition dans le pays. Ils se montrèrent d'abord dans des champs appartenant aux communes de Jászkisér et de Jászapáti, dans des localités séparées et sur une surface totale de I 200 arpents cadastraux environ (690 ha environ).

L'année suivante, on signala une autre apparition dans le comitat de Hajdí, entre autres localités, dans la prairie bien connue sous le nom de Nagyhortobágy (Hortobágy), propriété de la municipalité de la ville de Debreczen. L'infestation s'étendit bientôt sur 42000 arpents cadastraux (2I I69 ha environ).

Elle fut suivie les deux années suivantes (I906-07) de l'infestation des communes et des villes voisines de la ferme de Nagyhortobágy: Haidúböszörmény, Hajdúnánás, Balmazujváros, Hajdúszoboszló, Nádudvar, Kúnmadaras (toutes dans le comitat de Hajdú), Nagyiván (comitat de Heves), Tiszapolgár (comitat de Szaboles) ; une superficie de plus de 60000 arpents cadastraux (34528 ha environ) se trouva infestée. Toutefois le territoire occupé par les sauterelles comprenait le double, soit I20 000 arpents cadastraux (69056 ha environ). On constata aussi quelques apparitions peu nombreuses dans les comitats de Heves. (Mezököresd et ses environs, Aldebrö, Tiszafüred, Pély et ses environs), de Jásznagykúnszolnok (Nagykörii, Kisujszállás, ferme d'Écseg, etc.), de Bihar (Nagyléta et ses environs) et de légères taches dans d'autres comitats (Pest: 'Tahitótfalu, Uri, le champ de manœuvre de 1'artillerie près de Budapest), mais ces dernières apparitions furent sans importance. Le foyer d'infection principal était le Nagyhortobágy et les champs s'y rattachant, un peu vers le nord-ouest du territoire infesté pendant les an1ées I889-I893.

Les sauterelles ont encore causé des dommages sur le territoire hongrois en Igo8 et en Igog. Cette dernière année, elles apparurent à Na- 
gykörii, sur une superficie de 700 à 800 arpents cadastraux (403-460 ha environ).

Nous ajoutons, pour compléter les renseignements précédents, que les sauterelles se présentent actuellement en quantités plus ou moins grandes daus tous les terrains situés sur les rives de la Tisza et entre celle-ci et le Danube. La zone la plus importante où l'on peut rencontrer encore aujourd'hui ces insectes, est celle qui commence au nord par le comitat de Ugocsa (commune de Som, sur les bords du canal actuel de Szernye) et s'étend, en passant vers le sud, jusqu'à Nagybecskerek (comitat de 'Torontál); de l'ouest à l'est, les limites sont formées par les communes de Jászapáti et de Piispökladány, y compris la commune de Kaba. Selon toute probabilité, le fléau se renouvellera périodiquement en Hongrie.

En Roumanie, la première mention de graves apparitions de sauterelles enregistrée dans les archives de ce pays 1emonte à I 598 ; mais ces insectes s'y montrèrent certainement bien avant cette date.

Depuis lors, la Roumanie à payé, à diverses reprises, de très forts tributs aux sauterelles, qui y ont été particulièrement nuisibles en I640, I 708, I7I I, I7I6, I747, I749, I800-0I, I803, I8I2-I6, I820-22, I829-3I, I834-36, I 847 , I 850-5I, I859-6I, I865, I887, I894-I 900, I 902-03, I906-07.

Ce furent en premier lieu le nord et le sud de la Roumanie qui ressentirent les tristes effets de la présence des sauterelles; elles arrivèrent ensuite dans l'intérieur du pays.

Plus récemment encore (I III), ces orthoptères ont été observés dans le district de Romanatzi.

Le pays est encore aujourd'hui sous la menace du fléau, étant donnée surtout la possibilité d'invasions provenant des pays limitrophes, Russie et Bulgarie.

Depuis la fondation (I g02) de la première Station expérimentale d'Agriculture à Sadovo, de grands dégâts produits par les sauterelles ont été constatés pour la première fois en $\mathrm{Bulgarie,} \mathrm{au} \mathrm{mois} \mathrm{de} \mathrm{mai} \mathrm{rgo6,}$ exactement dans 1'arrondissement de Dobritch.

En I 907 (6 juin v. st.), ces insectes endommagèrent le village d'Odirné ; En I908, les villages de Pandakli (arr. de Silistra) et d'Armadja (arr. de Kourt-Bounar) ; la même année, au commencement du mois de juin, le champ d'expériences de la Station d'Agriculture de Roussé; en Igo8, également, le haras de "Klemintina " (arr. de Pléven). En Igo9, les sauterelles occasionnèrent des dommages dans les arrondissements de Pléven, Nikopol, Troyan, Béla-Slatina, Choumen, Ihtiman, Plovdiv, Kazanlik, Stanimaka, Haskovo et à Orhanié. En Igo9-Io, leur présence fut particulièrement notable au village de Dautlaré (arr. de Plovdiv). En IgI , la Station expérimentale de Sophia a été informée que les sauterelles étaient apparues à Brestovitza (arr. de Béla-Slatina).

Quant à la Gr èce, Hippocrate, Théocrite, Nicandre, Dioscoride, Galien, AlexANDRE de Tralies, etc, pour ne citer que ces auteurs, font déjà mention des sauterelles d'une manière plus ou moins précise et détaillée. 
Ce n'est pas seulement dans l'antiquité la plus reculée, mais aussi dans la suite que l'on a eu à constater et à déplorer en Grèce la présence de ces insectes. D'après des renseignements relativement très récents, les sauterelles causaient autrefois, de temps à autre, de nombreux dommages à l'agriculture du pays; elles apparaissaient en grande quantité pendant deux ou trois ans, puis elles disparaissaient pour réapparaître quelques années plus tard.

En IgIr, elles s'étaient tellement multipliées que 1'on craignit à un certain moment une catastrophe générale et la destruction complète des cultures. Elles avaient occupé presque tout le territoire, sauf quelques points de la péninsule et quelques îles. Elles ont fait une nouvelle apparition en I gI2.

ASIE. - I.es sauterelles ne paraissent pas existel dans le Borné o britannique du Nord.

Bien qu'ils fréquentent T i m c r (cfr., entre autres auteurs, AD. TARGIONI TozzetTI et L. REH), il ne résulte pas du rapport officiel envoyé à l'Institut international d'Agriculture que ces insectes aient quelque importance dans la partie portugaise de cette île.

De même, ils n'ont aucune valeur économique pour Hong-Kong et n'ont jamais pris, semble-t-il, les proportions d'un fléau sérieux au Si a m .

En I895, et même ensuite, des sauterelles sont apparues à Ceyla n, dans les districts de Kurunegala, Matale et Kadugannawa. A présent, toutefois, il ne semble pas, d'après le rapport parvenu à 1'Institut, que des espèces se réunissant en bandes et sujettes à migrations aient quelque importance dans l'île.

Occupons-nous maintenant des autres pays de 1'Asie où le fléau a été signalé d’une façon plus marquée.

Un des bons écrivains florentins, MATTEO PALMIERI, rapporte que déjà en I355 les sauterelles dévastaient Chypre. Si l'on ajoute foi aux faits enregistrés dans de vieux documents, cette île eut le même sort en I4II: le fléau prit même à cette époque des proportions tellement considérables, que les arbres atteints par la morsure des insectes restèrent privés de leur feuillage pendant trois ans. Dans la suite, les districts de l'île ont subi presque chaque année des dégâts de la part des sauterelles. F. Uitger et Th. Kотsснy parlent des grands dommages causés dans l'île par ces insectes en I865.

En I88I, entre autres années où les sauterelles continuaient à pulluler sur le territoire de l'île de Chypre, les pertes subies par les agriculteurs furent tellement considérables qu'on les évalua à plus de 80000 livres sterling ; l'année suivante la situation ne causa pas moins de préoccupations à 1'agriculture.

En I 884 , les sauterelles occupèrent en très grand nombre et sur une superficie de I9 $\mathrm{km}$ la plaine de Tchnigahi, près de Nicosie ; le fléau menaçait de ruiner complètement le pays, tellement il prenait de vastes proportions. Combattues par 1'Administration anglaise plus énergiquement encore qu'on ne l'avait fait à une époque antérieure récente (IS6S-70), les sauterelles 
ne se sont plus montrées en bandes depuis cette année-là ; bien que des individus isolés existent encore çà et là dans l'île, on peut dire qu'ils n'y soulèvent plus de préoccupations sérieuses, grâce aux mesures en vigueur.

En P e r s e, c'est surtout dans le sud que les sauterelles occasionnent depuis longtemps de grands dommages.

En $\mathrm{C}$ h in e, les chroniques ne font aucune mention spéciale de ces insectes jusqu'à la dynastie de Hai (20g av. J.-C.) ; on trouve cependant quelques allusions les concernant dans les vieux livres.

On commença à s'occuper des sauterelles à partir de la susdite dynastie. Elles ont surtout attiré 1'attention à l'époque de l'empereur Jon-T'Thien (I723) ; durant la dynastie Mantchou, sous le règne de l'empereur Djelon (I754) les provinces de Chen-Si et de Chan-'Ton furent visitées par le fléau. La province de Kang-Sou fut également atteinte mais dans des proportions bien moins grandes. A une époque plus récente, les dommages et les disettes causés par les sauterelles ont été meutionnés, entre autres, par ANDREOZZI et STEFANELII (I870) ; ce dernier qualifie de "désastre " la présence des sauterelles en Chine. Les renseignements font défaut pour ces dernières années.

En ce qui concerne le Japon, si nous nous reportons aux anciennes chroniques du pays, nous y trouvons mentionné que "lorsque les conditions météorologiques ne sont pas normales, des "inago" prennent naissance parmi les plantes de riz, causent de grands dommages et amènent parfois la famine : c'est un fléau venu du ciel ". Comme autrefois tout insecte susceptible de causer des dégâts aux récoltes de riz paraît avoir été généralement désigné sous le nom d' "inago ", il est impossible de se rendre compte aujourd'hui, vu la variété des insectes nuisibles au riz, si l'expression dont on usait à cette époque se rapportait aux vraies sauterelles.

Quoi qu'il en soit, il est certain qu'à une époque beaucoup plus rapprochée des vraies sauterelles ont été signalées au Japon; elles $y$ apparurent en nombre considérable en I 880 (I $3^{\text {ème }}$ année de Meiji) dans une partie de la province de 'Tokachi (région de 1'Hokkaido) et on les considéra alors comme venant du continent. Elles se multiplièrent rapidement et après avoir détruit toutes les cultures de la localité, elles dévorèrent la végétation des forêts et des plaines pour s'étendre ensuite dans toutes les directions. Elles apparurent de nouveau l'année suivante; leurs dommages s'étendaient sur une superficie de 40 "ri ") (I $57 \mathrm{~km}$ environ) de l'est à l'ouest, sur 20 "ri " (79 km environ) du nord au sud : la dévastation. fut complète ; on évalua l'étendue totale du territoire infesté à cette époque à plus de I60 "chôbu " ( 59 ha environ), y compris plaines et forêts. En I go5I $906\left(38^{\text {ème }}-39^{\text {ème }}\right.$ année de Meiji), les sauterelles ont fait leur apparition dans les îles d'Ogasawara (îles Bonin). De plus, elles se sont montrées chaque année en quantités plus ou moins importantes dans ces îles et elles occasionnent parfois encore des dommages dans d'autres paities du Japon.

La tradition veut que les sauterelles aient apparu il y a un grand nombre d'années dans l'Etat de Kélantan, situé dans la partie orientale de la péninsule de Malacca et qu'elles y aient occasionné de grands dommages. 
Des sauterelles ont été signalées aussi sur la côte orientale de la même péninsule, à Kuantan.

Dans les Etats Fédérés Malais, pays jeune en ce qui concerne le développement de l'agriculture, il n'existe aucun document écrit attestant d'une manière certaine la présence des sauterelles dans le passé ; d'autre part, les habitants sont incapables de fournir des renseignements personnels à cet égard, si l'on en excepte de rares et peu certaines allusions à une apparition de ces insectes qui aurait précédé les bandes signalées en IgI2 à Port-Dickson, dans l'Etat de Négri-Sembilan. De là, le nombre des insectes ayant augmenté, ils se répandirent peu à peu vers le nord, à partir de 1'année susmentionnée, et ils arrivèrent jusqu' à 'Tanjong-Malim dans l'Eitat de Sélangor, se tenant d'abord dans leur migration à une distance relativement faible des bords des voies ferrées et des routes.

En I9I3, et aussi en I9I4--au début de cette dernière année, elles furent signalées dans le Pahang - les sauterelles se trouvaient en nombre considérable sur le territoire des Etats Fédérés Malais. Dans l'ensemble, près de 7600 bandes furent détruites, en I9I4, dans les Etats de Sélangor, de Négri-Sembilan et de Pahang ; les plus éprouvés par le fléau furent Sélangor et Négri-Sembilan.

En I9I5, on n'a pas constaté d'éclosion dans le Pahang; on a détruit en tout 202 bandes dans le Sélangor et 607 I dans le Négri-Sembilan.

Après leur apparition dans les Etats Fédérés Malais, les sauterelles se montrèrent dans les $\mathrm{E}$ tablissements du Détroit, plus précisécisément à Malacca, et aussi, en quantité notable, dans 1'Etat de J o hor e . Pendant les mois de novembre et de décembre I9I4, 330 bandes avaient été déjà détruites daus ce dernier Etat; les sauterelles y furent également observées en I9I5, année pendant laquelle on détruisit I505 bandes.

A J a va, où les sauterelles ont produit des dégâts plus ou moins graves à différentes reprises, on eut à déplorer d'une manière particulière leur présence en 1887 , dans les résidences de Sémarang et de Rembang ; ces insectes occasionnèrent àussi de graves dommages dans 1'île en I9I4-I 5 .

En ce qui concerne l'Inde, on lit dans l'Hunter's Gazetteer que les sauterelles causèrent quelques dommages à Ahmédabad et à Broach, en I8I2, et qu'elles visitèrent Etawah en I $82 \mathrm{I}$. En I834, elles occasionnèrent des dommages à Kaïra et à Ahmédabad; en I843-4t Rawalpindi fut gravement atteint. En I863, le Pendjab et le Radjpoutana furent visités par les sauterelles qui y causèrent de grands dommages aux cultures. En I864, des rols furent signalés dans un district du Pendjab aux mois de juillet et d'août, et on les signala de nouveau l'année suivante pendant les mêmes mois. En I866, les sauterelles apparurent en petite quantité pendant le mois de juin et en $\mathrm{I} 867$, on aperçut un petit vol en juillet.

Fin I869, tout le Radjpoutana et le Pendjab furent occupés par de grands vols de sauterelles; elles furent signalées de nouveau en IS 72 daus plusieurs districts du Pendjab et, en I873, dans les districts de Jhelum et d'Amritsar. En I876-77 les sauterelles furent observées vers le mois de juillet dans le district de Hissar et on les aperçut de nouveau en I8 8 dans le DéraGhazi-Khan, le Hissar et le Radjpontana. D'après l'Hunter's Gazetteer elles ap- 
parurent aussi, en I878, dans le Kolaba. E11 I879, des vols furent signalés encore dans ces régions ; 1'année suivante le Déra-Ghazi-Khan et le Jeypur furent visités et les cultures d'été y subirent des dommages considérables.

La Présidence de Bombay fut visitée par les sauterelles en I882-83.

D'autres apparitions de ces insectes furent enregistrées dans 1'Inde pendant les années I889-90, I896-98, et aussi en I903-04 (Présidence de Bombay), en I904-06, en I907 (encore dans la Présidence de Bombay).

En septembre I903, les sauterelles occupaient dans l'Inde une superficie qui, selon toute probabilité, n'était pas intérieure à $64750 \mathrm{~km}^{2}$; pendant les mois d'hiver cette superficie se réduisit à $3 I 000 \mathrm{~km}^{2}$ et les insectes se répandirent ensuite sur une étendue mesurant au moins $562600 \mathrm{~km}^{2}$.

Certaines années, les vols sont tellement épais qu'ils arrivent à voiler la lumière du soleil. Les régions visitées par les bandes sont immédiatement dévastées, les arbres ne portent plus aucune trace de feuilles, les cultures herbacées sont dévorées à fleur de terre, et des trains sont quelquefois arrêtés par le passage des bandes sur les rails.

Quelques parties du Pendjab et du Radjpoutana septentrional sont particulièrement sujettes à la visite du fléau, car les bandes qui se sont formées dans le voisinage de ces régions s'y rendent fréquemment, deux, trois fois et même davantage avant de se subdiviser en bandes plus petites.

La présence des sauterelles a été ultérieurement constatée dans le Sind en IgI2; les deux années suivantes ces insectes visitèrent encore dans des proportions notables le Sind et aussi le Kathiavar et le Gujarat septentrional; en I9I5 des invasions ont été signalées dans les districts de Jalpaiguri, Darjeeling et Térai ; et on ne peut pas exclure le fait que, même par la suite et dans d'autres localités, ces insectes seront signalés comme nuisibles, attendu que leur diffusion est certaine sur une aussi grande partie du territoire de 1'Inde.

AFRIQUE. - Les orthoptères qui nous occupent seraient inconnus dans les possessions espagnoles du Golfe de Guinée, dans la Somalie italienne et aux Seychelles.

De plus les sauterelles sont considérées comme une rareté dans la Somalie anglaise; elles ont été incidemment observées comme nuisibles dans 1'Ougañda; elles ne produisent que des dommages insignifiants dans le Gouvernement politico-militaire de Rio.de Oro, vul le manque presque absolu de cultures.

La C y r é n a ï q u e eut déjà à souffrir de la présence des sauterelles, l'an de Rome 637, année où 1'ancienne Numidie fut aussi éprouvée durement par le fléau.

On trouve dans PLINE I' ANCIEN et dans STRABON des renseignements sur les sauterelles concernant la Cyrénaïque et la Libye au commencement de l'ère chrétienne. A une époque beaucoup plus rapprochée, les sauterelles furent observées en I890-9I en Cyrénaïque et durant la dernière de ces années, en Tripolitaine (Tripoli, Djébel Nefousa, Fezzan); elles furent également signalées sur la limite de cette région vers la fin de Igo6. Actuelle- 
ment, il paraît qu'on n'en trouve plus trace en Cyrénaïque et qu'elles n'ont pas une importance considérable en ce qui touche la 'Tripolitaine.

Dans les colonies anglaises de la Gambie et de la Côte de 1'Or, on n'a plus fait, semble-t-il, aucune mention particulière de ces insectes respectivement après 1893 et I898.

Au Togo - d'après les observations de HERoL, — des bandes furent observées pour la première fois, en janvier I892, à Agome, district de Misahöhe; des observations ultérieures nous apprennent que ces insectes y séjournèrent jusqu'en I8g6 et y causèrent des dommages. 'Toutefois, dans ces dernières années ils n'y ont produit aucun dégât.

Au dire des indigènes, le district de L,ome a été visité par les dernières bandes entre IgOI et Ig03 environ.

I a dernière apparition dans le district d'Anecho eut lieu en Ig03.

De nombreuses sauterelles furent aperçues en I9I2 à Nuatja et dans le district de Sokode près de Kamaa. Dans cette dernière localité, pendant le mois d'octobre, toutes les feuilles furent détruites en deux ou trois heures dans de petites plantations de cotonnier, et plus tard de la citronnelle qui venait d'être plantée fut complètement perdue en peu de jours.

Sauf les renseignements concernant les sauterelles, que 1'on trouve dans un ouvrage publié par le Rév. S. W. KöLIE en I 554, il semble que les autres apparitions de ces insectes, tant dans la Nigéria septentrionale que méridionale, dont on ait conservé un souvenir relativement précis, ne remontent pas à une date très éloignée; d'antre part, les apparitions de ces orthoptères paraissent avoir été plutôt rares dans tout le territoire, bien que peu de districts, même vers la côte, aient échappé probablement à leurs dommages.

Seuls les hommes d'un certain âge peuvent fournir des renseignements généraux au sujet de ces visites dans le pays. Les indigènes ont une conception plutôt vague de la chronologie, de sorte qu'il est difficile d'établir, du moins chez les Yoroubas, 1'époque à laquelle chaque apparition a réellement eu lieu. Un Yorouba de la classe des paysans, qui pouvait avoir environ trente-cinq ans, certainement pas plus de quarante, se rappelait, il n'y a pas bien longtemps, une de ces apparitions dans son district natal de Ilesha, province de Ibadan (Nigéria méridionale); comme on lui demandait de préciser l'époque à laquelle ce fait remontait, il répondit qu'il avait dî se passer, croyait-i1, une soixantaine d'années auparavant.

Dans les provinces septentrionales, territoire de Hausa, la rareté relative de ces apparitions, leur caractère redoutable et leurs tristes conséquences, car il semble qu'elles aient été généralement suivies de disettes, ont amené à les considérer, avec une terreur superstitiense, comme la conséquence ou le signe précurseur de quelque autre grand événement. Ainsi dans la province du Niger (Nigéria septentrionale), d'après les renseignements recueillis sur place, une apparition de sauterelles suivit la mort dı "Sariki » musulman (vers I898) ; cette même apparition fut considérée plus tard comme le présage de l'arrivée des Anglais en Ig0o. Dans de nombreux districts, il faut remonter à environ vingt ans pour avoir des renseignements sur la dernière apparition de sauterelles, mais, en I904, il y en eut une sérieuse danı 
le district de Yelwa, province de Kontagora. Une visite précédente avait eu lieu dans la même province en I897. La province de Zaria fut atteinte en I907. Les sauterelles n'ont pas visité Sokoto pendant au moins dix-sept ans. La province de Nassarawa eut à déplorer une visite en I9I 5 ; la précédente avait eu lien vingt ans auparavant.

Dans les provinces méridionales, il semblerait, d'après les rares renseignements dont on dispose, qu'une bande s'avança vers le sud jusqu'à Lagos il y a peut-être une quinzaine d'années environ. A cette époque Ibadan paraît avoir été également visitée, mais dans les provinces méridionales les apparitions de sauterelles semblent être encore plus rares que dans les provinces septentrionales. Une autorité indigène exprimait son opinion en disant qu'tun homme devrait être vienx pour avoir assisté dans sa vie à denx apparitions.

La majeure partie des visites faites dans le territoire par les sauterelles ont été de courte durée; la plus longue fut d'environ trois années consécutives; la superficie envahie tut relativement peu étendue, de sorte que si des disettes peuvent avoir été la conséquence des apparitions, les choses ne tardèrent pas à reprendre d'elles-mêmes leur cours normal.

Il est impossible d'établir l'étendue du territoire susceptible d'être infesté. De mémoire d'homme encore vivant, tous les districts des provinces septentrionales ont été probablement visités et on a le souvenir de visites dans les provinces méridionales, au sud jusqu'à Lagos. Il ne semble pas qu'il y ait eu d'infestation générale dans un groupe quelconque de provinces; les ațtaques ont été purement locales, bien que plus ou moins graves pendant leur durée.

Quant au Cameroun, depuis I883 la présence des sauterelles y fut enregistrée six fois en tout dans le territoire de Ngaundere, où 1'apparition la plus considérable eut lieu en I892. Les dommages furent alors importants, car les sauterelles furent suivies de la disette. Elles apparurent pour la dernière fois dans cette contrée en Igo8.

Ces insectes n'ont plus été signalés, parait-il, dans le territoire de Banjo depuis IgOI ou Ig03.

Dans le district de Yoko, où les sauterelles sont rarement apparues en grande quantité, les indigènes les ont aperçues il y a quelques années, par petites bandes d'une longueur et d'une largeur d'une vingtaine de mètres ; dans ces derniers temps elles n'y ont presque plus été observées.

Mais c'est dans les deux districts les plus septentrionaux de Garoua et de Mora que ces orthoptères ont été particulièrement nuisibles à 1'agriculture.

Dans le premier de ces districts, au commencement de la saison des pluies, après 1'ensemencement, on a aperçı récemment des bandes d'une étendue de plusieurs kilomètres carrés. 'Toutefois, d'après le témoignage des indigènes, les sauterelles ne se sont plus montrées à Pittoa depuis I90I.

Des bandes sont apparues fréquemment dans le district de Mora; en I907, les sauterelles se sont montrées en grand nombre à Dikoa et y ont causé de graves dommages.

Des observations concernant les sauterelles ont été faites à plusieurs 
reprises au Congo belge; les premières datent de I894. D'après les documents existants, des vols de ces insectes y ont toujours été signalés jusqu'ici.

En I894 exactement, plusieurs apparitions de sauterelles ont été constatées dans 1'Oubangui : le 4 mars à Mokoange et, en novembre, à Imese ; en 1895 des sauterelles ont été signalées à Zongo (Oubangui) et à Boma; en I896, ces insectes furent observés à Banzyville (Oubangui), à Boma et dans le district des Cataractes : à Kingila-Nord en novembre et à Kimuenza en décembre. D'autres vols ont été signalés à Katanga (décembre I898), dans la région du 'Tanganika (janvier I899), aux environs du lac MIoëro (novembre I900); en décembre I902, aux alentours d'Aba (Uele).

On peut dire, toutefois, qu'on ne s'est pas trouvé jusqu'à présent en présence d'une vraie invasion de sauterelles sur le territoire de la Colonie. Tous les vols signalés ci-dessus ne comprenaient en général que des nnées peu importantes que l'on arrivait facilement à disperser et même à détruire sans qu'elles aient pu produire des dommages irréparables. Dans quelques cas, cependant, comme dans la région de Kingila, en I896, et dans celle d'Aba, en r902, les cultures furent tortement atteintes et mêmes détruites; mais ce sont des cas isolés, intéressant des régions d'une superficie relativement faible. festés.

On ne saurait donner avec précision l'étendue des territoires in-

Des bandes de sauterelles ont été signalées de temps en temps dans différentes localités de l'Afrique orientale anglaise.

Ces insectes y firent deux apparitions vers la fin de Ig03 (Nairobi Farm, Voi); on les vit un peu plus souvent en Igo4 (Teita Hills, Voi, Nairobi Farm, Tsano) ; plus souvent encore en I905 (Mile IIo, Voi et Tsano, Ndii Hills, Kenani, Naivasha, Makindu, Simba, Nairobi, Kitui, Ravine, Athi River, Mile I35, Fort Hall, Muhoroni, Baringo, Kikuyu Station); les sauterelles firent deux apparitions en 1906 (Mwatate, Fort Ha11) et det1x également en rgos (Fort Hall).

Parmi les différentes apparitions, il faut en signaler particulièrement deux parmi celles enregistrées en 1905 . Au commencement de mai, toute la partie septentrionale de Baringo jusque vers Sugota fut dévastée par les sauterelles, qui n'auraient jamais, paraît-il, causé autant de ravages. Puis le 2 novembre, vers $x$ h 30 de l'après-midi, un nuée extrêmement étendue commença à passer au-dessus de la Station de Kitui; les sauterelles volaient près de terre et affectaient la forme d'une épaisse colonne haute d'environ $\mathrm{I} 5 \mathrm{~m}$ et couvrant une étendue de territoire large d'environ $\mathrm{I} 6 \mathrm{~km}$; elles allaient contre le vent avec une vitesse de $20 \mathrm{~km}$ à l'henre environ. A $5 \mathrm{~h}$ 30 de l'après-midi, les insectes passaient encore an-dessus de la Station; ils s'abattirent au coucher du soleil. Le lendemain matin, ils. reprirent leur vol et ils continuèrent à passer sur Kitui pendant 3 heures, puis la bande s'éloigna. Comme il n'y avait pas de cultures à cette saison, le district ne souffrit aucun dommage.

Depuis I908, les sauterelles n'avaient pas été, paraît-il, signalées dans l'Afrique orientale anglaise; cependant, par exception, dans la première 
moitié de IgI4 on les vit un peu partout dans le Protectorat, mais elles n'y firent que de petits dégâts.

A Maurice, les sauterelles qui ne causent actuellement de dommages à aucune culture, constituèrent par contre jusqu'en I770 un fléau périodique pour l'agriculture locale.

D'après les différents auteurs qui ont traité ce sujet, il semble que, avant cette date, les sauterelles avaient plusieurs fois menacé l'île de la famine et la victoire des agriculteurs sur les redoutables orthoptères paraît due à l'introduction d'un oiseau de la famille des sturnidés, le martin triste (Acridotheres tristis).

BERNARDIN DE SAINT-PIERRE, qui visita Maurice en I768, écrivait: "Les insectes les plus nuisibles sont les sauterelles; je les ai vues tomber sur un champ de cannes vierges, s'accumuler sur la terre de plusieurs pouces d'épaisseur et en dévorer la verdure dans une nuit. C'est l'ennemi le plus redoutable de l'agriculture ».

En I766, les agriculteurs se réunirent afin de remédier anx conditions agricoles de 1'île; ils décidèrent que des mesures spéciales devaient être adoptées pour la destruction des animaux. nuisibles et principalement des sauterelles.

En conséquence, il fut publié une ordonnance en vertu de laquelle tout planteur était tenu de détruire chaque année un certain nombre de rats, de singes, etc. et 4 "pounds ) ( $\mathrm{I}, 800 \mathrm{~kg}$ environ) de sauterelles par esclave en sa possession.

Plus tard, en I768, comme le nombre des sauterelles ne paraissat pas diminuer, une nouvelle ordonnance fut émise, qui obligeait tous les planteurs à coopérer à une destruction plus complète de ces insectes, en contribuant avec la dixième partie de leurs esclaves à une "chasse générale " qui fut ouverte à cette époque.

Mais, on constata plus tard que toutes ces mesures ne suffisaient pas à arrêter le fléau et que l'A. tristis introduit dans l'île en' I762 par le comte de Maudave s'était largement multiplié et détruisait en quantités notables toutes les espèces d'insectes et en particulier les sauterelles récemment écloses; on prit donc immédiatement des mesures pour protéger cet oiseau si utile à l'agriculture.

Une ordonnance fut émise en octobre I768; elle menaçait d'une grave peine pécuniaire (500 livres sterling $=$ I2 6 Io fr environ) quiconque faisait la chasse à l'oiseau ou à son nid ou tenait cette espèce en captivité ; la peine était plus grave en cas de récidive. Ces mesures protectrices furent confirmées par le paragraphe 6 du règlement $n^{0}$ I 84 du 7 mai I770.

On put constater cette année-là que les sauterelles avaient grandement diminué.

CÉRÉ, directeur du Jardin botanique de Pamplemousses, pouvait affirmer en I777 que 1'Acridotheres avait, en quelques années, délivré l'île du plus grand des fléaux, celui des sauterelles. Et CHARPEN'Tter DE Cossrgry pouvait écrire que "l'Ile de France était exposée autrefois aux ravages des sauterelles; on n'en a pas vu depuis l'année I770. On prétend que les martins, espèce qu'on y a importée de l'Inde et qui s'y est multipliée 
étonnamment, les ont détruites. Il est certain que ces oiseaux s'en nourrissent avec avidité lorsque les sauterelles ne font que de naître avant qu'elles aient des ailes \%. MAGON DE SAINT-EILIER confirmait le fait en écrivant: "En I770 cessèrent les ravages des sauterelles, véritable fléau qui, durant plusieurs années, avait arrêté le progrès de 1'agriculture. La destruction de ces insectes est attribuée aux martins ».

Jusqu'ici, nous avons examiné les régions de l'Afrique où les sauterelles constituent un souvenir historique, une rareté ou un fléau n'ayant une importance considérable que par exception.

Il n'en est pas de même dans les pays suivants appartenant presque tous à l'Afrique septentrionale et méridionale.

La présence des santerelles au M a roc avait déjà été constatée, d’après АвоU-MIohaMmed-SALAH-BEN-EL-ALIM, en 97 I, 987, I220, I227 et I279 après J.-C. ; elles occasionnèrent souvent dans le pays durant ces années-1à d'épouvantables disettes. Elles y apparurent aussi, semble-t-il, pendant le $\mathrm{XVI}^{\mathrm{e}}$ siècle ; elles y causèrent ultérieurement des dommages énormes et il en fut de même en I7IO, au dire de Moura-AHMED-Eí-Mourbi, dans la plaine et dans la montagne ; ces dégâts se renouvelèrent encore en I760-68, d'après G. Hös', en I778-80 (DE CHÉNIER), en I799-ISO0 (J.-G. JACKSON), en ISI3-I5 (HEMrso), en I866-67, en I80I, année où les ravages atteignirent, comme précédemment d'ailleurs, des proportions extrêmement graves; ils se produisirent avec le même caractère en I 897.

I a famine causée par les santerelles pendant la période comprise entre I778 et I780 occasionna la mort de milliers de personnes.

Enn I828 (deuxième quinzaine de juillet), Guyox observa à plusieurs reprises la présence de sauterelles sous les murs et aux environs de Ceuta, en face de Gibraltar.

En IgI t, les premiers vols de sauterelles furent signalés le 20 norembre au sud d'Agadir. Une invasion eut lieu aussi en IgI5.

On peut affirme1 que la question des sauterelles présente toujours un intérêt particulier pour le Protectorat français au Maroc.

Le souvenir, très fréquent, des sauterelles en A $1 \mathrm{~g}$ é $\mathrm{x}$ i e s'associe le plus souvent à celui d'invasions vraiment effrayantes quant à leur étendue et aux conséquences qu'elles ont entraînées, disettes, faim et maladies contagieuses.

L'ouvrage imposant et bien conuu des spécialistes de J. KüNCKEL D'HERCULAIS contient un exposé historique très détaillé des apparitions des sauterelles en Algérie.

Abstraction faite de très anciennes mentions des sauterelles en Algérie, contentes implicitement dans les œuvres de TiTE-LIVE, de JULIUS OBsEQUENS, de SAINT-AUGUSTIN et d'Orose, et où il est question des dommages immenses causés par ces insectes en Numidie et en Cyrénaïque, 1'année de Rome 637 (I25 ans av. J.-C.), et abstraction faite également de l'apparition presque certaine de ces orthoptères sur le territoire algérien pendant le IVI ${ }^{e}$ siècle, on conserve parmi les souvenirs les plus précis et les moins récents des ravages produits en Algérie par les sauterelles 1'affirmation de MouLAAHMed-E, -Mourbi, d'après laquelle le sud-algérien fut ravagé en i7Io 
en même temps que le Maroc. D'autres invasions eurent lieu sur plusieurs parties du territoire algérien en 1724-25, d'après ce qu'en rapportent 'THoMAs SHaw et J.-A. PExssonnex; puis en I784, d'après le souvenir qu'en laissa le botaniste DESFONTAINES; en I8I3-I5, d'après les observations de RFiNAUDO' 1 , et encore en I 822 et en I 824 .

Après la prise d'Alger (I830) les apparitions de sauterelles deviennent périodiques. On cite les invasions de I844-46, I848-50, I864-67, I868-72, I $873-76$, I877, I882-89, I890-93, I894-97, I899-I902. Iit on ne peut pas dire que la dernière date citée marque la fin de la série déjà si imposante des apparitions de ces insectes en Algérie, car ils ont éveillé et éveillent toujours de très vives préoccupations. En effet, ils ont été signalés, par exemple, en Igo8. Vers la fin de I9I4, les sauterelles se trouvaient sur la limite du Tell. En IQI5, elles ont été observées le long du littoral et encore en plus grand nombre à l'intérieur. A Djelfa (dép. d'Alger), + 500 doubles décalitres d'insectes avaient déjà été détruits durant la première moitié de cette année-là. Dans le département d'Oran, les sauterelles étaient répandues un peu partout, principalement à Saïda. La situation était plus grave dans le département de Constantine.

Quant à l'étendue du territoire infesté, on peut dire qu'en général pendant les différentes invasions, les sauterelles ont été plus ou moins observées dans les trois départements d'Alger, d'Oran et de Constantine.

Nous avons eu l'occasion de parler un peu plus haut de l'invasion de sauterelles q11i rendit ces insectes tristement célèbres en Numidie I25 ans av. J.-C. La peste qui suivit cette formidable apparition ne détermina pas seulement une forte mortalité parmi les animaux, mais elle sévit aussi sur les habitants spécialement aux environs des villes de Carthage et d'Utique, situées toutes les deux - comme on le sait - dans le pays qui forme aujourd'hui la 'T un is : e. Les principales invasions subies par le pays après cette dernière furent celles de I663-d'après ce qu'en rapporte ABouSALEM-EL-AÏACHI - de I845, I866, I874-75, I877, I888, I89 I, I897. En janvier I907, un premier vol de sauterelles s'abattit sur les territoires militaires; les mesures prises ne purent pas empêcher ces insectes d'occuper successivement tous les territoires. Le contrôle de Gafsa et son annexe de Tozeur luttèrent toute l'année pour empêcher autant que possible les ravages; le contrôle de Maktar lutta du commencement de mai à la fin de juin et se rendit maître de la situation. Enfin, les contrôles de Kairouan et du Kef furent aussi touchés, bien que faiblement.

A partir du mois de janvier I go8, des vols de sauterelles apparurent sur les territoires militaires et l'invasion gagna progressivement tout le territoire de la Régence; les sauterelles firent encore leur apparition en Igog dans les territoires militaires et dans le sud-twnisien. Les régions atteintes furent principalement les territoires militaires, les contrôles civils de Gabès, Gafsa et Kairowan.

Une autre invasion eut lieut en IgIo ; elle intéressa le contrôle civil du Kef (caïdat des Franchiches) et le nord-tunisien. I,es sauterelles ont encore été signalées en quantité formidable très récemment (I9I5) en 'lunisie ; d'après des renseignements transmis de Monastir, au commencement de 
juin de cette année-là, les sauterelles ramassées se montaient bien à I 8069 sacs et le ramassage continuait toujours ; les dommages causés aux diverses cultures étaient très graves. Presque tout le contrôle de Sfax a été atteint : 22020 sacs de sauterelles ont été recneillis et 6940 litres d'œufs ont été ramassés.

Si à propos de sauterelles il est question de l'Eigypte, le souvenir de la Bible et des "plaies" qu'elle décrit se présente immédiatement à 1'esprit.

Nul n'ignore en effet la mention particulière que l'Exode fait de ces insectes: nous y lisons qu'à la septième plaie représentée par un orage épouvantable qui dévasta une partie de la récolte en Egypte, vint s'ajouter 1'apparition d'un immense vol de sauterelles qui acheva la destruction de tout ce que la grêle avait épargné.

Nous pouvons ajouter d'une manière générale qu'en Egypte les sauterelles ont apparu aussi par la suite en quantité considérable bien qu'à des intervalles irréguliers. Les renseignements les plus récents que l'on possède au sujet d'importantes invasions dans le pays concernent les années I89I, I904 et I9I5.

Durant la première des trois années susmentionnées les sauterelles occupèrent le territoire situé entre Girgeh et la Méditerranée (Alexandrie); elles furent surtoụt signalées dans les provinces de Béhéra, Ménoufieh, Charkieh, Galioubieh (Basse-Egypte) et dans celles de Grizeh et de Fayoum (Haute-Egypte).

En I904, les localités les plus particulièrement atteintes furent les environs de Salhieh, de Bilbeis, Suez, Matarieh, Bassatine, quelques endroits de Béni-Souef et du Fayoum et les terres de Maady.

Enfin, en I9I5, les sauterelles ont encore plus ou moins gravement endommagé la Basse- et la Haute-Egypte (Béhéra, Gharbieh, Ménoufieh, Dakahlieh, Charkieh, Galioubieh, Guizeh, Fayoum, Béni-Souef, Minieh, Assiout, Guirgueh, Kének, Assouan, etc.).

Depuis l'époque de la domination italienne, la première apparition de sauterelles en Erythrée a eu lieu en I905; cette année-là, toutes les récoltes furent complètement détruites. En Igo6, les produits de la Colonie furent réduits de moitié : il en fut de même en I907. L'année suivante les sauterelles firent une nouvelle apparition. Des dommages moins graves ont été enregistrés en IgI2 et en I9I3. Depuis ce moment, jusqu'en I9I5, les sauterelles ont continué d'attirer l'attention et elles ont même soulevé de plus ou moins graves préoccupations ; la dernière année citée elles ont causé, entre autres, des dommages dans les zones de l'Adi Ugri et de Saganeiti.

On a constaté que toute la Colonie est facilement infestée durant le cours de l'année solaire.

On ne sait rien de précis au sujet de la première apparition et des apparitions successives des sauterelles à Sier ra-Leone. 'Toutefois-à ce que l'on affirme - ces insectes y constituent un des pires fléanx contre lesquels doivent lutter les agricultenrs du pays.

C'est en I89o que remonte la prenière mention des santerelles dans l'Afrique orientale allemande; cette année-là, Stuminaxx 
observa, durant le mois d'octobre, de larges bandes à Umpeke. On n'en avait jamais entendu parler une vingtaine d'années avant cette date. Ia période des apparitions successives dura de I893 à Igoo; elles atteignirent leur plus grande intensité en I895-96 et elles allèrent ensuite en décroissant. Plus tard, on signala encore de grosses bandes dans le Nord de la Colonie, de I903 à Ig06; il y en eut encore dans l'Ousanbara en I903-04 et en I905.

L'apparition de grandes et de petites bandes a été signalée plus récemment (IgI3-I4) dans la région nord-est du pays.

Il résulte des renseignements que l'on possède actuellement que les sauterelles se répandent sur le territoire entier; il faut excepter tout au plus de la superficie atteinte une fraction de l'Ounyamouezi.

Impossible de préciser l'époque à laquelle remonte la première apparition des sauterelles dans la Rhodésia méridionale. Quoi qu'il en soit, on en a un souvenir certain à partir de Igo6. Le développement atteint 1'année suivante par 1'invasion fut vraiment extraordinaire; qu'il suffise de dire, pour en donner une idée, que I4 I09 bandes furent détruites; quelques-unes de ces bandes atteignaient des proportions gigantesques, calculées en kilomètres; les dommages turent naturellement très graves. Les sauterelles furent également signalées en quantités notables en Ig08 et en Ig09. Après IgIo, on les vit en petite quantité à Iírancistown, en I 9I5. 'Tout le territoire de la Colonie est susceptible d'être infesté.

Abstraction faite de ce qui a pu avoir lieu à une époque antérieure dans 1a Province de Mozambique, on dispose de renseignements certains sur les sauterelles à partir de Igoo, lorsque pour commencer à les combattre, la "Companhia de Moçambique" proposa d'inscrire à son budget une première somme qui fut affectée l'année suivante à la défense de l'industrie sucrière sur le Zambèze, où les sauterelles causaient de graves dommages; la lutte fut alors entreprise contre ces insectes, entre autres localités, à Séna sur le Zambèze et dans la zone de Manica. Ėn I902-03, I904-05, I906-07 et même plus tarê, ils se trouvaient encore dans la région du Zambèze.

En I903-04, des bandes furent signalées dans une localité située sur les bords du Mozambique; en rg04-05 les sauterelles furent particulièrement nuisibles dans la zone de Séna.

Une plantation de canne à sucre située dans la région du Zambèze et d'une étendue de I 092 ha environ faisait espérer une récolte de 4572 t environ de sucre, en I906-07; on n'en retira que I 828 t à cause de la présence des sauterelles; ce fut une perte de $2744 \mathrm{t}$ environ valant $605 \mathrm{fr}$ chacune.

En I907-08, l'organisation officielle de la lutte fut entreprise dans la Province d'une manière pratique ; afin de pourvoir anx opérations le Gouvernement de la Province, d'accord avec le "South African Central Locust Bureau " de Prétoria, avait dès 1907 , inscrit à son budget nne contribution financière spéciale.

Fn juillet Igo8, une bande était signalée dans les parties inhabitées de la zone méridionale du Zambèze ; aux mois de juillet et d'août de la même année des bandes furent aperçues aussi dans la partie la plus septentrionale de la Province, près du lac Nyassa.

A partir du mois d'octobre suivant, les santerelles infestèrent peu à peu 
d'une manière particulière les districts de Zambèze, Inhambane et LourençoMarques.

Mais, de toutes les régions de la Province, celle du Zambèze fut la plus gravement atteinte. En. I907-08, I4 t d'œufs furent détruites dans une seule plantation de canne à sucre de I 355 ha; dans la même région un tiers et même deux tiers de la récolte d'environ 9 Io 000 cocotiers furent perdus par suite des dégâts causés par les sauterelles en I907-08; les planteurs éprouvèrent alors un dommage financier que l'on peut estimer à I 89 I $605 \mathrm{fr}$.

A Lourenço-Marques, 50 bandes furent détruites à la même époque.

En I909, une bande de sauterelles endommagea les plantations de cocotiers appartenant à la "Companhia Colonial de Busi " près de Beira.

La même année, au mois d'avril, on aperçut nne nuée desauterelles dans la partie septentrionale de Gaza; au mois de mai suivant on la vit dans la région occidentale de Lourenço- Marques et, en juin, un peı plus au sud.

Ce même mois de juin, les sauterelles furent signalées dans le district appartenant à la "Companhia do Nyassa ", le plus septentrional de la Province.

D'avril à décembre ces insectes furent observés au 'Zambèze, d'abord dans les localités septentrionales du district, puis dans les plaines de Marral, dans les plantations de canne à sucre de Caia, Mopea et Marromeu, qui furent considérablement endommagées, surtout à Caia.

Au mois de décembre I909, les sauterelles furent encore signalées dans la Province sur la limite de la Rhodésia méridionale.

Après cette date elles se sont encore montrées, bien qu'en petites quantités, sur plusieurs points de la Province: en IgIo, dans les districts d'Inhambane (à Cumbana, Maxixe, Homoíne, Panda, Panga, Morrumbene, Inharrime et Jacobene), du Zambèze (à Mopea) et de LourençoMarques (à Chibuto); en IgII, dans le district d'Inhambane (à Homoíne, Massinga, Panga, Jacobene, Inharrime, Mocodoene); en IgI2, elles apparturent à nouveatı dans les districts d'Inhambane (à Jacobene, Mocodoene, Homoíne, Maxixe, Massinga et Morrumbene) et de Lourenço-Marques (à Bela Vista, Catuane, Esstatuene, et Catembe) ; en I9I3, elles se montrèrent encore dans les districts d'Inhambane (à Panga, Jacobene, Massinga, Homoíne, Panda) et de Lourenço-Marques (à Catuane, Salamanga) et, au commencement de I9I4, elles firent une nouvelle apparition dans ce dernier district, à Catuane.

C'est à l'année Igo6 que remontent les renseignements rraiment dignes de foi sur la présence des sauterelles dans le Sud-Onest africain allemand, où une apparition importante fut aussi enregistrée en I 907. Les sauterelles y ont fait de nouvelles visites en IgoS et en Igng-Io ; en janvier I9I0, elles furent signalées dans les districts de Rehobotlı, Bethaniein, Aus et près de Grootfontein.

Quant à l'étendue du territoire infesté, on peut dire que les sauterelles sont répandues dans toutes les régions de cette Colonie.

Depuis longtemps ces insectes ont attiré l'attention et causé des alarmes dans 1'Union de l'Afrique du Sud. D'après Chas.-P 
Lounsbury, des marins qui visitèrent la vallée de la Table les y observèrent bien avant la colonisation de Cape 'lown par les Hollandais et VAN RIEBEEK constata des dégâts produits par les sauterelles pendant le premier été qu'il passa dans cette contrée (I653). 'THEAL, dans son histoire de 1'Afrique du Sud, les mentionne comme ayant occasionné de nouveaux dommages dans la petite Colonie du Cap en I687. Il est vraisemblable que les sauterelles y produisirent d'autres dégâts quelques années plus tard, mais 'THEAL rapporte que la Colonie fut épargnée de i695 à la fin de I746, époque à laquelle elles se montrèrent encore dans la vallée de la 'Table ; leur nombre était tellement grand que 1'air en paraissait rempli et en quelques jours il ne resta plus rien de mangeable dans la vallée; les feuilles des arbres ne furent pas même épargnées. I es dommages furent également considérables dans le territoire voisin et le prix de la viande doubla dans la Colonie, car une grande quantité de bétail mourut de faim. Le fait stuvant se rattache à cette invasion: vers I742, 1' "East India Company » avait entrepris la construction d'un môle dans la baie de la Table et les agriculteurs qui portaient leurs produits au marché devaient transporter aussi des pierres pour le môle. Environ quatre ans après, c'est-à-dire à la fin de I746, on se vit forcé d'arrêter les travaux, car la végétation ayant été détruite par les sauterelles et les produits agricoles étant venus à manquer, on ne pouvait plus transporter de pierres et la construction du môle fut suspendue pour un temps indéfini.

A une époque postérieure, les dévastations causées par les sauterelles furent fréquentes dans l'intérieur de l'Afrique du Sud. Une période d'abondance de ces insectes paraît avoir commencé vers I797, onze ans après la fondation de Graaf-Reinet, et avoir duré jusqu'en I808. En I824, toute la contrée fut visitée par les sauterelles; elles descendirent des centres de colonisation les plus septentrionaux vers le sud, jusqu'à Bedford et le fléau n'en disparut que vers I83I ; pendant cette période, les sauterelles furent observées à Kuruman, en I826, au dixe du missionnaire J.-B. Moffat.

D'autres apparitions eurent lien en I842-I854. Au commencement de I843, les sauterelles causèrent des dommages au Cap et dans les districts environnants. Quant à la Colonie du Natal, y compris le Zoulouland abstraction faite d'une tradition indigène d'après laquelle le Natal aurait été envahi par les sauterelles à une époque plutôt reculée - ces insectes 'existaient réellement dans le Zoulouland sous le règne du roi Mpande (I850) et il est certain que leur première apparition dans la région avait eu lieu quelques années auparavant ; de I 847 à I 853 , les sauterelles infestaient le Natal où elles se montrèrent particulièrement abondantes en I 852-53. On raconte, à propos de la quantité considérable de ces insectes qui avaient envahi le pays, que de grosses branches d'arbres se rompaient partois sons le poids des sauterelles.

De I862 à I876, il y eut une autre période d'apparitions ; dans ce temps-là, on observa des sauterelles entre autres à Kenhardt, en I869. Puis, la contrée fut considérée comme délivrée des sauterelles pendant quatorze ans, bien que quelques apparitions aient été signalées durant cette période dans les districts du centre situés plus au nord dans le Cap. 
A partir de I8go - en I89I, par exemple, elles visitèrent le Transvaal et la Colonie du fleuve Orange - les sauterelles ont été signalées çà et là tous les ans dans l'Union jusqu'en Igog; on estime que cette date mit fin à un autre cycle ou à une série de cycles de grandes apparitions. En I907, on détruisit, dans le 'Transvaal seulement, I 5000 bandes, dont beaucoup avaient plusieurs milles d'étendue. I, dommage total que les sauterelles y ont produit en I907-08 fut d'au moins un million de livres sterling.

Après une période d'absence presque absolue, les sauterelles apparurent en quantités considérables en IgI3-I.4 dans l'Afrique du Sud, où elles furent encore signalées en I9I4-I5. En I9I4, dans la partie centrale du Cap, on détruisit dans I34 propriétés, 768 bandes, comprenant, selon 1'évaluation qui en fut faite, 389000000 d'insectes.

D'une manière générale, on peut dire que les territoires des quatre Colonies, qui constituent actuellement les quatre Provinces de l'Union de l'Afrique du Sud, ont été plus ou moins éprouvés par les incursions des sauterelles; elles se sont montrées parfois simultanément ou à peu près dans deux ou trois de ces Colonies.

Comme témoignage de l'importance et de la gravité qu'ont eues dans le passé et à une époque récente les invasions de ces orthoptères, tant dans 1'Union que dans d'antres régions voisines, nous citerons le fait, assez éloquent par lui-même, de la création, en rgo6, du "South African Central Locust Bureau ", dont le siège était à Prétoria, capitale du Transvaal; son but était de pourvoir, avec la coopération des divers pays intéressés, à rendre plus prompte et plus efficace la lutte contre les sauterelles. Nous parlerons ailleurs de l'organisation de ce "Bureau», de son fonctionnement et des résultats de son activité pendant les quatres années de son existence.

Les sauterelles sont connues aussi aux Canaries. Elles existent de temps immémorial dans l'île de Hierro.

Nous avons des renseignements assez précis sur les anciennes apparitions des sauterelles dans ce groupe d'îles entre autres pour les années I507, I588-89, I645, I649, I660, I759, I778, I779, I800, I876, etc.

En Igoo-or, on constata dans tonte l'île de Hierro une superficie infestée de 2000 ha. Au commencement de septembre Ig02, les sauterelles apparurent dans l'île de Ténériffe. İ'existence dı fléaı dans l'île de la Goméra fut signalée en premier lien le $\mathrm{I}^{\mathrm{er}}$ mai IgIo.

Durant l'automne et 1'hiver de cette année-là, l'étendue de territoire infesté dans les îles de Hierro (à Valverde) et de la Goméra (à Arure, Vallehernıso, Olajéró et San Sebastián) mesurait en tout 3I2,50 ha.

Au printemps de IgI I, le santerelles ont été signalées dans les localités déjà atteintes pendant 1'antomne et 1'hiver de IgIo et à Adéje et 'Tanque, dans 1'île de 'Ténériffe.

Pendant 1a campagne de IgII-I2, la présence de ces orthoptères fut encore constatée dans les trois îles et dans les zones susmentionnées, sur une étendue totale de 467,86 ha.

Ein Igr2, ils apparurent pour la première fois dans 1'île de la GrandeCanarie (à San Bartolomé de Tirajana, à 'Téjéda sur wne étendue de 354 lıa, et aussi à Arténara). 
Ces insectes ont été signalés ultérieurement anx Canaries pendant la campagne I9I3-I4.

AMÉRIQUE. -- En ce qui concerne l'Amérique du Nord, il convient de déclarer tout d'abord que les sauterelles ne semblent pas être connues aux Bermudes.

A Terre-Neuve, elles ne sont plus considérées actuellement comme un fléau, bien qu'elles aient pris cet aspect une fois, vers I862 ; on n'en a plus entendu parler depuis dans la Colonie comme d'insectes dévastateurs.

Par contre, au Can a d a, les sauterelles ont causé périodiquement des dommages les années suivantes: I8I8-I9; I857-58; I864-65; I87I-75 et, de temps en temps, même dans des années très récentes: en I9I2, I9I3, IgI 4 et IgI5 elles étaient extrêmement nombreuses et nuisibles dans 1'Ontario et dans le Québec.

De grandes pertes produites par ces insectes ont été constatées plusieu1s fois aussi dans d'autres parties du Canada, et particulièrement dans la Colombie britannique et au Manitoba.

On n'a plus à redouter, en général, des invasions de sauterelles migratrices couvrant une vaste étendue de territoire aux Et ats-Unis. Toutefois, durant la première moitié du XIX'e siècle, d’immenses dommages furent produits dans cette contrée par des bandes de sauterelles qui se développèrent sur les versants orientaux des Montagnes-Rocheuses, à une hauteur variant de 3000 à 6000 "feet» (900 à I $800 \mathrm{~m}$ environ) aul-dessus du niveatı de la mer. On a aussi le souvenir d'une invasion considérable en r 766. Une autre, très grave, eut lieu en I8I 8 et, en I853, de vastes bandes apparurent dans l'Idaho, dans l'Utah, dans le Dakota du Nord et le Dakota du Sud. La première apparition générale réellement désastreuse, dont on ait un sonvenir authentique et détaillé, eut lieu durant les années I86.4 et I865 ; cette invasion occupa une vaste partie des Etats et des 'Territoires de 1'Utah, de Montana, des deux Dakotas et du Colorado, la moitié septentrionale du Nouveau-Mexique et le Nebraska, Jowa et Minnesota. Depuis cette époque jusqu'en I874, de fréquentes apparitions de ces insectes furent signalées dans les Etats occidentaux et nord-occidentaux.

Durant la dernière année citée, eut lieu une des invasions les plus désastreuses dont le souvenir soit resté dans l'histoire de cette contrée: presque toute la partie du territoire située à l'ouest du $94^{\mathrm{e}}$ méridien et à l'est des Montagnes-Rocheuses fut visitée par les sauterelles ; les pertes qu'elles causèrent alors furent évaluées à 50000000 de dollars (259 I25 000 fr). L'invasion dura encore l'année suivante et l'on évalua le dommage occasionné dans un seul Eitat, celui de Missouri, à I 5000000 de dollars (76 $735500 \mathrm{fr}$ ). Le 3 mars 1877 , le Congrès des Etats-Unis autorisa la nomination d'une Commission de trois entomologistes expérimentés, C.-V. RiLizy, A.-S. PACkARD et Cyrus Thomas ; ils furent chargés d'entreprendre des recherches et de faire un rapport sur les ravages des sauterelles dans les Etats et les Territoires occidentaux. Les résultats de ces recherches sont contenus dans deux 
rapports successifs de la Commission, comprenant les années I877 et I87879; ces rapports constituent une ceuvre vraiment monumentale et représentent sans donte la publication américaine la plus complète qui ait jamais été tentée sur ce sujet.

Depuis $I 878$, a ucune grande invasion générale de sauterelles migratrices n'a été signalée dans la contrée et aucune apparition locale importante 11'a plus en lieu depuis I89I. Cependant en I899, une sérieuse apparition d'une forme non migratrice fut signalée sur le territoire situé entre les MontagnesRochetises et le Mississippi et y causa de grands dommages. A l'est du $100^{e}$ méridien, des apparitions locales de diverses espèces non migratrices ont été signalées plusieurs fois. La partie de la Californie dont le climat est semi-aride a eu aussi à souffrir gravement du fait des sauterelles, dont le souvenir remonte à I722, date la plus ancienne rappelée pour les EtatsUnis. En I 855 , une apparition notable eut lieu dans ce territoire; il y en eut une autre en 1873 .

Des dommages plus ou moins graves ont été signalés également dans les années qui suivirent immédiatement la dernière année citée, et cela jusqu’à ces temps derniers. La superficie infestée par les sauterelles en IgI3, dans le Nouveau-Mexique seulement, variait de I 000 à I $300 \mathrm{~km} 2$; les pâturages et les cultures y furent dévastés en grande partie. Des troupeaux de bétail qui paissaient ordinairement dans cette zone durent être éloignés de I8 à $2 \mathrm{I} \mathrm{km}$ pour trouver facilement à pâturer et ne purent retourner à leurs abreuvoirs habituels qu'à des intervalles variant de 24 à 56 heures. Des trains de marchandises et de voyageurs furent arrêtés à plusieurs reprises, surtout de la moitié de mai au premier juin, par les sauterelles qui encombraient les rails en quantités énormes.

Etant données les conditions extrêmement favorables à la propagation des sauterelles offertes par toute la contrée, il est probable que le territoire des Etats-Unis sera toujours plus ou moins soumis aux attaques de ces insectes.

Nous bornant à ne citer que les exemples les plus récents, nous signalerons les apparitions suivies de dégâts qui eurent lieu, entre autres, en IgIo au Colorado; en IgI I, au Minnesota et au Kansas, qui fut atteint aussi l'année stivante; en I9I3, il y en eut de notables au Nouvean-Mexique, au Kansas, dans les Etats d'Oklahoma, de New-Hampshire, de Vermont; de moins graves dans 1'Arizona, le T'exas, le Mississipi, le Wisconsin, le Michigan, le Wyoming ; en I9I4, on en constata dans 1'Orégon et dans l'Etat de NewYork.

Quant à 1'étendue des territoires infestés, ont peut affirmer en général que les dommages les plus importants produits par les sauterelles aux EtatsUnis se limitent à la région située à l'ouest du $90^{\circ}$ méridien, bien que de nombreuses apparitions locales de nature plus ou moins grave aient eu lieu daus les E,tats occidentaux du centre et même dans les,orientaux. La NouvelleAngleterre en particulier a considérablement souffert certaines années. Toutefois, ces apparitions n'ont rien de comparable avec les ravages cansés par des formes migratrices dans les régions plus occidentales des E.tats-Unis pendant la première moitié dı XIXe siècle. 
Les invasions de sauterelles aı M ex i que, d'après des renseignements puisés à diverses sources, remontent anx temps les plus reculés. On peut préciser à cet égard les dates suivantes, qui indiquent la durée des maniiestations les plus importantes du fléau et le temps qui s'est écoulé entre elles: I6II-I8; I63I-38; I66I-67; I690-97; I73I-38; I77I-79; I80I-04; I830-36; I854-59; IS7I-77; I882-86; I887-IgI3.

Les zones de territoire infestées et les dommages qui y furent cansés ont été souvent des plus considérables. Une disette, conséquence de la présence des sauterelles, affligea le pays entre I738 et I739. L'invasion de I854 s'étendit aux Eitats orientanx et méridionanx de la République, comprenant les Etats de Guerreto, Colima, Jalisco, Guanajuato, Véra Cruz et peut-être aussi quelques autres situés plus au nord.

Ein juin I888, une large bande envahit le district de Téhuantépec et occasionna de grands dommages à Comitancillo, Chihuitán, Tlancotépec et dans la ville chef-lieu du district; au mois d'octobre de la même année, les sauterelles furent très abondantes à Quérétaro et, a11 mois de décembre suivant, à Técoman et à Colima; d'octobre à décembre Igo2, elles apparurent en grand nombre dans les Eitats de Guanajuato et de Michoacán, où elles causèrent de très graves pertes.

Le Yucatan a été fréquemment et gravement atteint. En I 87 I, les sauterelles s'abattirent sur 1'Hacienda de San Ignacio, entre Progreso et Mérida. En I882, les sauterelles visitèrent la région de Maxcanú dans le Yucatan, la région de Huatusco, dans l'Etat de Véra Cruz et la région située au nord du Guatémala; en I905, ces insectes visitèrent de nouveau à peu près les mêmes localités. Ces invasions ont été insignifiantes au début; puis, le fléau n'ayant pas été exterminé à temps, les sauterelles n'ont pas tardé à se multiplier surtout dans le Yucatan, et elles dévastèrent bientôt toute la péninsule.

Quant à 1'Amérique centrale, il ne semble pas que 1'on ait eu à déplorer de ravages occasionnés par les sauterelles dans le Honduras britannique; on se rappelle toutefois que ces insectes apparurent en I 885 sur la limite septentrionale de la Colonie en exemplaires isolés, dont le nombre n'augmenta pas et qui perdirent bientôt toute importance. Des individus isolés furent rencontrés également dans le district de Stann Creek, dans la partie méridionale de la Colonie.

Ėn I80o, sinon auparavant, la présence des sa11terelles fut enregistrée da1s la République de Sa1va dor, exactement à Santa Ana. Fin I852, ces insectes envahirent les départements de San Vincente, La Paz, San Sa1vador et Sonsonate. D'autres invasions enrent lien en I 856 et en I883. Vers la fin de IgI4, tous les départements de la République ont été envahis et de grands dommages constatés dans les différentes cultures.

A Costa-Rica, ces orthoptères envahirent en I659 la vallée d'Aserrí; en I73I, on constata leur présence à Bagaces; une autre invasion eut lieu en I774. En I800, les sauterelles apparurent à Alajuéla et à Hérédia ; on en reparle encore en I804. En I852, on les vit de nouveaù à Alajuéla, où deux années plus tard, elles occasionnèrent de plus grands dommages qu'ailleurs. Ėn I 876 , elles envahirent plusieurs localités de la province de 
Puntarénas; elles éveillèrent aussi des préoccupations dans la République l'année suivante. Eilles furent signalées ensuite en IgI4 dans la province de Guanacaste et enfin, en I9I5, le pays a été largement envahi sur ses deux versants jusqu'à sa frontière avec la République de Panama. A l'entrée de la province d'Alajuéla, la bande envahissante formait une nuée compacte occupant une superficie de plus de $20 \mathrm{~km}^{2}$.

Quant à l'Archipel des Indes occidéntales ou Antilles, les sauterelles semblent être inconnues aux îles $\mathrm{Bahama}$.

L'île de Cuba n'a jamais éprouvé de dommages de leur part bien que ces insectes y aient été signalés.

A la J a mä̈que, où leur existence est connue, on n'a pas souvenir qu'ils aient été considérés comme pouvant occasionner des dommages aux cultures de l'île.

Bien qu'existant dans l'île d'Hä̈t, on affirme que les sauterelles n’ont jamais fait d'apparition dans la République Dominicaine.

Eilles sont généralement répandues à $\mathrm{P}$ orto-Rico, mais on ne leur y attribue pas une grande importance économique.

L'apparition des sauterelles ne paraît pas avoir été signalée jusqu'à présent aux petites Antilles dan.s les possessions danoises (îles de St.-Thomas, St.-Jean et Ste.-Croix).

Ces orthoptères ne se montrent qu'en assez petit nombre à Antigua. Il en est de même pour Montserrat, pour la Dominique, pour Ste.-I, ucie, St.-Vincent, pour la Barbade, la Grenade, pour Tobago et Curaçao.

A St.-Christophe (St.-Kitts), les sauterelles apparaissent en quantités suffisantes pour attirer l'attention, mais seulement par intervalles et pendant de courtes périodes.

Elles acquièrent une plus grande importance pour l'île de la 'Trin i té. En effet, on a des renseignements certains sur une grande invasion qui, en I 885, s'abattit sur Icacos et, pett après, sur Cha ca cha ca re - petite île voisine de la Trinité — et Monos, autre île située entre Chacachacare et la Trinité ; vers la fin de la même année, une apparition ent lieu à Carénage, au nord-ouest de la Trinité, à environ I2 km de Port-d'Espagne.

En rgog, les sauterelles furent l'objet d'observations dans le district d'Icacos.

Trente ans après la première date susmentinnnée, c'est-à-dire en I I 5 , elles furent signalées en très petites quantités dans l'île de P a tos (située entre Chacachacare et le Vénézuela), en nombre encore plus réduit à Chacachacare même et à Monos ; on en captura un exemplaire vivant à Port-d'Espagne.

Au mois de juin IgI6, une bande de sauterelles s'abattit sur Patos; ensuite un petit nombre de ces insectes fut remarqué dans la partie sudorientale de la Trinité.

Dans 1'Amérique du Sud, les sauterelles ne paraissent pas être connues aux îles F a $1 \mathrm{k} 1 \mathrm{a}$. d. 
Elles n'ont pas une grande importance économique dans la G u y a ne hollandaise (Surinam).

La première attaque de sauterelles dont on ait connaissance dans la Guyane anglaise eut lieu en I886; cette année-là, une vaste superficie cultivée du comté de Berbice fut dévastée. Des dommages aux cultures ont été signalés quelquefois, mais ils n'ont eu que rarement une grande importance économique. Récemment (I9I5), une apparition de sauterelles a encore été constatée à Corentyne Coast, Berbice.

Toutes les zones cultivées de la Guyane anglaise sont exposées à éprouver des dommages de la part de ces insectes.

Les traditions recueillies dans les localités envahies font remonter au commencement du XIXe siècle 1'apparition des sauterelles au Pérou; mais ce n'est que depuis seize ans que ces insectes y sont en permanence, avec des alternatives d'intensité dépendant des divers facteurs qui exercent sur eux leur influence.

L'étendue du territoire atteint est relativement faible, car jusqu'à présent les sauterelles n'ont pas été au delà de certaines parties des départements de Huancavélica, Ayacucho, Apurimac et Cuzco, dans les vallées du versant oriental de la Cordillère des Andes.

Au Brési1, les sauterelles ont attiré l'attention à diverses époques.

Dans ces dernières années, elles ont été signalées à plusieurs reprises, en particulier dans 1'Etat de Rio Grande do Sul. M. Carlos Moreira rapporte que, voyageant précisément dans 1'Eitat de Rio Grande do Sul, il eut l'occasion d'observer, en septembre I905, une invasion nombreuse qui s'abattit sur Cacéquy où une partie de la bande commença immédiatement à causer des dommages. Le train dans léquel M. MoreIRA voyageait avançait avec difficulté, car les corps écrasés de ces insectes faisaient patiner les roues sur les rails devenus glissants.

D'après le même entomologiste, les sauterelles apparurent encore depuis dans l'Etat de Santa Catharina, près de la localité appelée Macacos, à une trentaine de kilomètres de Lages.

En I905-06, on a constaté de graves dommages dans les Eitats de Paraná et de São Paulo.

Pendant toute 1'année I908, on dut lutter âprement contre les sauterelles dans 1'Etat de Rio Grande do Sul.

En Igog, année où eut lieu une des plus grandes invasions qui affligèrent le Brésil, les Etats de São Paulo, Minas Geraes, Rio de Janeiro et Espirito Santo furent ravagés.

Au mois de juin I9Io, en plein hiver, les sauterelles furent signalées à Campina Grande (Eitat de Parahyba do Norte), sur une superficie de 6 "leguas » $(33,4 \mathrm{~km})$ du nord au sud, sur I6 $(89 \mathrm{~km})$ de 1'est à l'ouest, soit une étendue de 96 "leguas quadradas" (298 I28 ha).

Des bandes de sauterelles causèrent aussi de graves dommages sur divers points de l'Etat de Rio Grande do Sul au mois de juillet et d'août IgI I cet Etat eut également à déplorer de grands dommages en I9I4-I5.

Une partie du 1'Etat de Sâo Paulo fut envahie aussi en I9I5-I6.

En général, la partie du territoire brésilien plus ou moins soumise aux 
dégâts des sauterelles comprend les Etats de Matto Grosso, Rio Grande do Sul, Santa Catharina, Paraná, São Paulo, Rio de Janeiro, Espirito Santo et Minas Geraes.

En Bolivie, les sauterelles sont un vrai fléau pour l'agriculture des départements de Tarija, de Chuquisaca et d'une partie de celui de Santa Cruz. Elles furent signalées en 1898 et en I905-08 dans le territoire de la République. Les renseignements les plus récents les concernant datent de IgI4-I5; elles occasionnèrent de très grands dommages dans presque tout le territoire des provinces d'Arce, Avilés et O'Connor. Dans le département de Tarija, les produits agricoles ont été réduits à la quatrième partie de la récolte espérée. Le bétail a également beaucoup souffert du manque de pâturages, presque entièrement détruits.

Quant au Chili, on a des renseignements certains concernant I8gI 92, années où la destruction des sauterelles exigea une dépense de plus de I50 000 "pesos » (283 500 fr).

Elles y furent encore signalées en Igo6; elles envahirent alors la zone de Lonquimai, Malacahuello et Curacautin.

Les annales de la République Argentine n'enregistrent que bien peu de renseignements avant la première moitié du XIXe siècle, en ce qui concerne les sauterelles et leurs invasions. Cela dépend, en partie, de l'absence de tout Service officiel chargé d'organiser la lutte contre le fléau et d'étudier les modalités des invasions, et, d'autre part, du fait que les industries agricoles consistant presque exclusivement dans l'élevage $\mathrm{du}$ bétail paissant sur de vastes étendues de prairies naturelles, les dommages que purent causer les orthoptères furent de peu d'importance et ne troublèrent nullement l'économie nationale.

Mais le moment vint où 1'agriculture commença à se développer et à ressentir de plus près les effets des sauterelles; alors, 1'indifférence générale du public et de la presse vis-à-vis du fléau disparut et les Gouvernements provinciaux d'abord, puis le Gouvernement national se préoccupèrent de trouver les moyens d'atténuer les dommages dont souffraient les zones cultivées.

Ce fut en I 897 que 1'on entreprit des études précises; elles permirent de connaître les mœurs de la "langosta » qui dévastait le pays et la manière dont s'accomplissaient ses iuvasions périodiques.

L'étendue du territoire de la République infesté par ces orthoptères est excessivement variable d'une année à l'autre et l'importance des invasions présente des alternatives périodiques d'augmentation et de diminution.

Parmi les grandes invasions, on rappelle particulièrement celles qui correspondent au printemps et à l'été de $1875-76$, où les bandes envahissantes avançant vers le sud dépassèrent le $35^{\circ}$ de latitude méridionale, et celles de I879-80 qui déterminèrent le Gouvernement de Santa Fé à publier la première loi de "Defensa Agrícola".

Les dates indiquées plus bas donnent une idée claire des invasions subies à partir de I897, époque à laquelle les Services de "Defensa Agrícola " reçurent, dans la République, une organisation pratique; les dates sont sui- 
vies des chiffres correspondant aux progrès de 1'agriculture; ces progrès sont la raison déterminante des lois qui ont été successivement publiées et de l'effort croissant fait en vue d'une défense toujours plus efficace de la production nationale menacée par les sauterelles.

\begin{tabular}{|c|c|c|c|c|c|c|}
\hline Années & & & & $\begin{array}{l}\text { Territoire envahi } \\
{\mathrm{en} \mathrm{km}^{2}}^{2}\end{array}$ & & $\begin{array}{l}\text { Superficie cultivée } \\
\text { eu } \mathrm{km}^{2}\end{array}$ \\
\hline I $897-98$ & . . & . . & & I 400000 & & 39738 \\
\hline I898-99 & $\therefore \cdot$ & . . & . & 8I0 000 & & 4405 I \\
\hline I899-90 & . . . & . . & . & 553000 & & 46377 \\
\hline Ig00-OI & . . & . . & . & 80000 & & 52279 \\
\hline I90I-02 & $\cdots$ & . . & . & 68000 & & $55 \mathrm{I} 72$ \\
\hline I902-03 & . . & . . & . & $\dot{I} 20000$ & $i$ & 68606 \\
\hline Ig03-04 & $\cdots$ & . . & . & 500000 & & 79624 \\
\hline I904-05 & . . & . 。 & . & 600000 & & 83236 \\
\hline I905-06 & . . & . . & . & 873000 & & 94875 \\
\hline I906-07 & . . & . . & . & I I 40000 & & $79 I 06$ \\
\hline I907-08 & $\cdot \cdot$ & . . & $\bullet$ & I 307000 & & I02 569 \\
\hline I908-09 & . . & . . & $\cdot$ & I 320000 & & II2 346 \\
\hline I909-I0 & . . & . . & . & I 350000 & & I08 607 \\
\hline I9I0-II & . & . . & . & $89 \mathrm{I} 000$ & & II7 737 \\
\hline IgII-I2 & . . & . & . & 204 I52 & & I29 80 OO \\
\hline I9I2-I3 & . . & 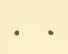 & . & $I_{4} 87 \mathrm{I}$ & & I36 7I 4 \\
\hline I9I3-I4 & . . & . & & $75^{8} 5$ I 7 & & I 40 I $5^{8}$ \\
\hline I9I4-I5 & . . & . & $\cdot$ & I 000000 & & I36 oII \\
\hline
\end{tabular}

En février I9I5 - pour ne citer qu'une date très récente - on a détruit en Argentine $34797762 \mathrm{~kg}$ de sauterelles, parmi lesquels étaient compris 34755 I57 kg d'insectes non ailés; on ne détruisit pas d'œufs. En mars, le total s'élevait à $654880 \mathrm{~kg}$, y compris I7 $480 \mathrm{~kg}$ d'œufs ; par contre on ne détruisit pas d'ailés.

Les invasions de sauterelles ont eu lieu de tout temps au $\mathrm{P}$ a r a g u a $\mathrm{y}$ : mais on croit généralement - et cela paraît être confirmé par des documents historiques, il est vrai très imparfaits et assez obscurs - qu'autrefois les invasions étaient moins fréquentes et moins nuisibles qu'elles ne le furent plus tard. La dernière invasion notable fut celle de I907.

Tout le pays peut être atteint, à l'exception de la région de l'est, dont les eaux se dirigent vers le Haut-Paraná, qui ne l'est que rarement. Les régions sont d'autant plus exposées au fléau qu'elles se trouvent situées plus à l'ouest et plus au nord. Eitant données cependant les conditions naturelles du pays et la richesse de sa végétation spontanée, malgré la fré- 
quence des invasions, le Paraguay n'est pas habituellement sujet à des dommages aussi graves que ceux que l'on constate dans d'autres pays voisins.

On ne connaît pas exactement la date des premières invasions des sauterelles en Uruguay; il est 'toutefois très vraisemblable qu'elles remontent à une époque fort reculée.

Des renseignements plus précis au point de vue chronologique ont été recueillis pour des années bien plus rapprochées de nous. Les sauterelles furent signalées, en effet, dans les départements du littoral pendant les années I890-9I, I892-93, I894-95. Les dégâts produits dans cette période ne sont pas comparables à ceux constatés en I896, année où 1'invasion prit un caractère vraiment alarmant et éveilla la préoccupation de tous les habitants de la République qui crurent tout d'abord que le bien-être du pays se trouvait profondément compromis.

L'invasion de Igo6-07 fut également très grave: les sauterelles se répandirent dans les départements d'Artígas, Rivéra, Salto, Paysandú, Río Negro, Tacuarembó, Durazno, Treinta y Tres, Cerro Largo, Soriano, Colonia, San José, Florés, Florida, Minas, Rocha, Canélones ; elles occupèrent approximativement tout le territoire de Salto, Paysandú et Colonia, excepté pour ce dernier la première section (Colonia) et elles envahirent presque complètement les départements d'Artígas, Rivéra, Río Negro; 1'invasion atteignit des proportions moins grandes à Tacuarembó (plus de $I 7000 \mathrm{~km}^{2}$ ), Durazno

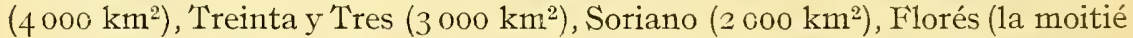
du département), Canélones (36 $\mathrm{km}^{2}$ environ), San José $\left(5-8 \mathrm{~km}^{2}\right)$; la superficie occupée à Minas était relativement insignifiante ; il y eut très peu de sauterelles à Rocha.

Les dommages les plus graves furent constatés dans les départements d’Artigas, Paysandú, 'Tacuarembó, Rivéra, Salto et Filorés.

Les départements de Maldonado et de Montévidéo ne furent pas envahis.

En IgI4, une grande invasion se répandit dans les départements de Paysandú, Salto, Río Negro, Soriano, Artígas, Colonia, Florida, Durazno, Tacuarembó, Rivéra, San José, Florés, Canélones, Montévidéo, etc. Le fléau sévit avec intensité sur la République aussi en I9I5.

Nous ne quitterons pas l'Uruguay sans signaler l'initiative prise par son Gouvernement au sujet de la réunion de la Conférence internationale de la "Defensa Agrícola ) dont nous parlerons plus loin et qui eut lieu à Montévidéo au mois de mai I9I3; nous signalerons plus spécialement à ce propos la Convention stipulée à cette occasion entre 1'Argentine, la Bolivie, le Brésil, le Paraguay et 1'Uruguay relativement à la recherche des foyers initiaux de l'espèce de sauterelles qui exerce ses ravages dans ces pays de 1'Amérique du Sud.

OCFANIE. - Les sauterelles sont connues aux îles $\mathrm{Ha}$ a a $\ddot{i}$, mais on ne leur y attribue pas une grande importance.

D'après les renseignements que $1^{\prime}$ on possède, il en est presque de même pour 1'Australie occidentale, pour 1'Australie méridionale - oì l'on ne se rappelle que deux apparitions de peu d'importance signalées 
dans la période qui comprend les vingt-cinq dernières années - et pour la Tasmanie.

Le $\mathrm{Q} u$ e e n $\mathrm{s} 1 \mathrm{a}$ d a été très éprouvé à diverses reprises par des bandes de sauterelles, qui en attaquent périodiquement les cultures.

Ces orthoptères ont occasionné de graves dommages, en I883 et pendant la campagne suivante, à Victoria Mill, dans les plantations de canne à sucre, qui furent également attaquées, en Igor, près du fleuve Herbert; et, en I904, ils menacèrent cette culture aussi à Childers, dans le Queensland méridional. De janvier à juin IgI2, les sauterelles ont été signalées dans le Queensland septentrional.

En I873, les districts sud-occidentaux de la Nouve11e-Ga11es du Sud furent visités par ces insectes; de nombreuses et graves infestations ont été signalées pendant vingt ans environ, jusqu'en I9I0, dans de vastes zones de cet Etat ; ce tut une perte de plusieurs milliers de livres sterling. I,es sauterelles causèrent en effet de graves dommages à l'intérieur du pays et dans les plaines du sud pendant les années I890-0I. On les vit durant les étés de I899 à Ig02 dans les districts occidentaux de Parkes et de Condobolin au sud vers le fleuve Murray: tout fut détruit. En I907, elles causèrent de grands dommages aux pâturages dans le district de Singleton; il en fut de même, par intervalles, depuis cette dernière année jusqu'au mois de mars rgo8 dans plusieurs localités; elles apparurent dans quelques districts occidentaux pendant les mois de mars et d'avril I907, et elles se répandirent du fleuve Darling à l'est vers les fleuves Bogan et Macquarie; aux mois de septemb̉e et d'octobre I907, 1es sauterelles furent observées à Dandaloo, Trangie, Narromine, Nevertire, Coonamble, Dubbo, etc. ; d'octobre à décembre dans la vallée du fleuve Hunter.

D'après BATH, les sauterelles furent signalées pour la prenière fois dans l'Etat de V i c t oria, en I 848 , peu de temps après que les colons eurent occupé les plaines septentrionales et occidentales; on lit dans les journaux de l'époque que d'immenses bandes ailées descendirent des districts septentrionaux et occasionnèrent de grands dommages dans de nombreuses localités: elles volèrent pendant des journées entières sans discontinuer audessus de Sandhurst (Bendigo) se dirigeant vers le sud.

Une nouvelle apparition grave eut lieu en I862; elle fut suivie d'une autre en I870. 'Trois ans après les sauterelles arrivèrent en grandes bandes au sud jusqu’à Bendigo. En I876, elles abondaient de nouveau dans les plaines septentrionales.

I1 y eut également de graves infestations en I89o et I89I.

A Swan Hill, sur le fleuve Murray, en Igoo une personne qui se rendait à cheval à Kerang passa à travers une bande de sauterelles dont elle estima la longueur à plus de $9 \mathrm{~km}$ et demi.

Au mois de décembre I907, près d'Albury, sur le fleuve Murray, un grand nombre de sauterelles voyageaient vers l'est à une vitesse d'environ II $\mathrm{km}$ à 1'heure en dévorant toute-la verdure. I a luzerne souffrit gravement; un enclos de pàturage d'une vingtaine d'hectares fut détruit en quelques minutes.

A Kilmore, en I907 également, les sauterelles se montrèrent en telle 
abondance que le service du chemin de fer en souffrit. Un train de Bendigo à Melbourne fut arrêté entre Pyalong et High Camp par ces insectes dont des myriades envahirent les rails, y formant une couche de $15 \mathrm{crn}$. Les roues de la locomotive écrasèrent les sauterelles et les réduisirent en une bouillie visqueuse qui empêchait les roues d'avoir prise sur les rails. Le train s'arrêta au bas d'une montée. Comme il était très chargé, on le divisa en deux; la locomotive partit avec la première partie du convoi composé de wagons contenant des ovidés et laissa en arrière les fourgons à bagages et les voitures de voyageurs. Près de Moranding la locomotive et les wagons à bétail furent arrêtés de nouveau par des bandes qui avaient envahi les rails et le convoi ne put arriver à Kilmore qu'avec un retard considérable.

Bien qu'on manque de données précises, on ne s'éloigne pas de la vérité en disant que 5I 800 ha environ du territoire de Victoria ont eu occasionnellement à soufffrir de l'attaque des sauterelles. On n'a pas fait une est1mation des dommages qu'elles ont produits à l'agriculture, mais ils doivent se monter à plusieurs centaines de milliers de livres sterling.

Dans certains districts septentrionaux de la Nouvelle-Zé1ande, on constate souvent des dégâts considérables par suite de l'attaque d'une espèce de Locustida. 


\section{ESPECES DE SAU'TERELLES NUISIBLES OBSERVEEES DANS LES DIVERS PAYS CONSIDËRÉS}

Nous avons cherché à réunir dans la liste suivante toutes les espècés d'orthoptères désignées habituellement, avec plus ou moins de raison, sous le nom de sauterelles (Phasgonuridae et Locustida) et reconnues pour nuisibles à des degrés divers aux cultures des pays considérés dans le chapitre précédent.

Néanmoins, les différentes sources auxquelles il nous a été donné de puiser ne nous ont pas permis d'établir, pour quelques-uns des pays en question, à quelles espèces plus ou moins nuisibles il était fait allusion : cela dépend peut-être de ce fait que, vu la rareté des apparitions dans ces contrées, leur durée éphémère et plus spécialement encore le peu d’importance des dégâts constatés, on n'a pas éprouvé la nécessité d’approfondir la.connaissance systématique de l'agent ou des agents dévastateurs.

L'astérisque placé devant les noms des localités, joints en regard des noms scientifiques des sauterelles, sert à indiquer d'une façon plus précise sur quels points du globe chacune des espèces a causé ou cause encore les dégâts les plus importants.

Toutes les fois que nous avons pu le faire, nous avons reporté, à côté des noms latins des orthoptères énumérés, le ou les noms vulgaires correspondants.

Pour la nomenclature et l'ordre de succession des espèces reportées dans la liste, nous avons cru bon de nous én tenir à l'ouvrage bien connu de W. F. KIRBY, déjà cité ; cependant, afin d'éviter toute équivoque, nous avons, lorsqu'il a été nécessaire, fait suivre les noms adoptés pour chacune des espèces, des synonymes sous lesquels elles sont généralement connues.

\section{PHASGONURIDE}

Gryllacris spp.

Drymadusa dorsalis Brullé (D. spectabilis Stein).

Pachytrachelus striolatus Fieb. $P$. gracilis Brunn.

Pholidoptera chabrieri Charp. $P$. littoralis Fieb.
Java.

Chypre.

Autriche: Carso gorizien.

$\begin{array}{lll}\eta) & y \\ \eta & \eta\end{array}$


Pholidoptera aptera Fabr.

Chelidoptera albopunctata Goeze (Platycleis grisea Friv.).

Tettigonia verrucivora Linn. (Decticus verrucivorus Serv.).

$T$. albifrons Fabr. (D. albifrons Serv.).

Phasgonura viridissima Linn.(Gryllus [Tettigonia] viridissimus; Locusta viridissima Fabr.).

P. caudata Charp. (Locusta caudata).

Conocephaloides maxillosus Fabr.

C. nitidulus Scop.

Mecopoda elongata Linn.

Cyrtaspis scutata Charp.

Orphania denticauda Charp. (Polysarcus denticaudus Fieb.).

Pecilemon ornatus Schmidt

$P$ elegans Brunn.

Barbitistes yersini Brunn.

B. ocskayi Charp. et Grav.

Barbitistes spp.

Isophya pyrenea Serv. (I. campsoxipha Brunn.).

Lcptophyes boscii Brunn.

Elimae chloris Haan
Autriche: Carso gorizien.

Italie (" locusta brizzolata ", " locustone $)$.

Autriche: Carso gorizien ("kleščence $)$.

Bulgarie: village d'Odirné.

*Portugal.

Espagne.

*Italie ("locusta », " locusta brizzolata ", "locustone", "locustone brizzolato" ou "porraiolo").

Autriche: Carso gorizien ("kleščence $»$ ).

Canaries (Iles).

Algérie ("bou sag" des Arabes). En français, "dectique à front blanc \%.

Portugal.

Italie (" cavalletta verde ). ,

Autriche : Carso gorizien ("kobila »). Malte.

En allemand, "grosses grünes Heupferd"; en français, "grande sauterelle verte” et, à tort, " cigale".

Autriche: *Dalmatie.

*Guyane anglaise.

Italie.

Mozambique: Vilanculos.

Java.

Autriche: *Carso gorizien.

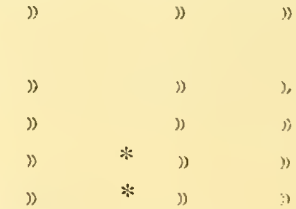

Bulgarie: village de Brestovitiza (arr. de Béla-Slatina).

Autriche: * Dalmatie.

Bulgarie.

Carso gorizien.

Autriche: Carso gorizien.

Java. 


\section{LOCUSTIDE}

Parateitix frey-gessnerii Bol.

Acrydium sp. Acrida turrita Linn. (Truxalis nasuta Fabr.; Tryxalis nasuta Fisch.). Truxalis brevicornis Linn.

Orphulella punctata De Geer Scyllina gregaria Sauss. (Plectrotettix gregarius Brumn.).

S. schistocercoides Rehr.

Dociostaurus maroccanus Thunb. (Gryllus maroccamus; Stauronotus maroccamus Stål; Gryllus critcialus Charp.).
D. brevicollis Eversm.

Gomphocerus sibiricus Linn.

Stenobothrus fischeri Eversm.

S. rubicundus Germ.

S. vittifrons Walk. Arcyptera fuscus Pall.

A. brevipeninis Brunn.
Porto-Rico.

Autriche : Carso gorizien. Java.

Argentine (.tucura »).

Porto-Rico.

* » »

Brésil : Matto Grosso.

Allemagne ("marokkanische Wanderheuschrecke ").

France: * Camargue ("criquet marocain ", "starironote marocain", "le marocain ").

*Portugal.

* Espagne ("verdadera langosta española ", " marroquí »).

* Italic (" cavalletta del Marocco ), " cava!letta maroccana ), "grillo crociato", "grillastro crociato", "locusta crociata").

Autriche: * Dalmatie.

*Hongrie.

Roumanie: distr. de Romanațî.

Serbie : dép. de Požarevac.

*Bulgarie.

*Grèce.

*Chvpre.

* Canaries (Iles).

Maroc, Protect. fraıç. (" Dierad-elAdami » en arabe).

Algérie.

Tunisie.

Tripolitaine.

Hongrie.

Suisse: Engadine.

En français, "criquet sibérien ». Autriche: * Carso gorizien.

») $)$

Australie: Victoria.

Autriche: *Wiener Wald; * Carso gorizien.

Enı français, "criquet bariolé». Autriche: * Carso gorizien. 
Omocestus petraus Bris.

Stauroderus bicolor Charp.

S. bignttulus Linn.

Chortippus pulvinatus Fisch.-Waldh.

C. albomarginaius De Geer

C. dorsatus Zett.

C. parailelus Zett.

C. curtipennis Harr. (Stenobothrus curtipennis Scudd.).

Elopolus tamulus Fabr. (Epacromia tumulus Jacobs. et Bianchi).

Chortoicetes pusilla Walk.

C. terminifera Waik.

Cammuia pellucida Scudd.

Celes variabilis Pall.

Oedaleus nigrofasciatus De Geer (Acrydium nigrofasciatum).

Oe. senegalensis Krauss

Gastrimargus sundaicus Sauss.

G. subfasciatus De Haan

Locusta pardalina Walk. (Pachytyius sulcicollis Stål; $P$. capensis Sauss.).
Autriche: * Carso gorizien.

Hongrie.

Autriche: * Carso gorizien.

$$
\text { ) ) 》 }
$$

Hongrie.

Autriche: Carso gorizien.

" Korneuburg.

Canada ("short-winged locust").

Java.

Australie: districts du centre et spécialement dans la partie occidentale de $1 \mathrm{a} *$ Nouvelle-Galles "du Sud ("smaller plain tocust »). Australie: de l'intérieur du pays à 1'Australie méridionale (districts septentrionaux et occidentaux); *Nouvelie-Galles du Sud : districts septentiionaux, occidentaux et méridionaux; Victoria : districts septentrionaux, occidentaux et méridionaux ("wandering plague locust" ou "larger plain locust)!.

Canada: *Colombie britamique ("pellucid locust»).

*Etats-Unis ( $y \in$ yllow-winged grasshopper ", "yellow-winged locust ", "pellucid grasshopper ").

Bulgar:e.

Italie (" grillo giallo »).

Hongrie.

Chypre ("common destructive 10cust $)$.

En français, "criquet panaché ». Congo belge.

Australie: Nouvelle-Galles du Sud (Montagnes-Bleues et territoires côtiers de l'est, entre autres le district de * Singleton). Connu sous le nom de "eastern plague locust". Java.

Timor.

*Rhodésia méridionale («brown locust ").

Mozambique ("gafanhoto cinzento", "gafanhoto pardo"). 
Locusta pardalina Walk. (Pachytylus sulcicollis Stål; P. capensis Sautss.).

L. migratoroides Reiche et Fairm. (Pachytylus migratoroides Sauss.; $P$. migratorivides Jacobs. et Bianchi).

L. australis Sauss. (Pachytylus aitstralis).

L. migratoria Linn. (Acrydium migratorivm De Geer; Pachytylis migratorius Fieb.).

L. danica Linn. (Pachytylus daricus Auriv.; Oedipoda migratoria Burm.).
Sud-Onest africain altemand («braune Heuschrecke », "sudafrikanische Wanderheuschrecke ").

Union de l'Afrique du Sud: *Transvaa1; *Orange; Nata1; *Cap ("brow11 locust", "old locust", ( sma11 locust "), "yellow locust", "khaki locust »).

Japon ("taiwan batta »).

Congo belge.

Afrique orientale allemande.

Nouvelle-Zélande.

Australie: districts côtiers du Queensland [*Childers, dans 1e Queensland mérid.] et de la Nouvelle-Galles du Sud (" large mottled locust ", "large northern locust 》); * Victoria ("common Victorian locust »).

*Allemagne (" europäische Wanderheuschrecke $)$.

Autriche: *Moravie; près de Budweis ; Istrie.

*Hongrie ("wandorsáska », "sereges sáska »). [XIX ${ }^{\text {ème }}$ siècle)].

*Roumanie.

Malte.

*Japon: prov. de Tokachi (rég. de l'Hokkaido) et îles Ogasawara [îles Bonin]. Nom vulgaire: "batta". Mexique (?).

Salvador

En français, "criquet voyageur».

Grande-Bretagne: Ecosse; Ang!eterre.

Hollande.

Belgique.

Allemagne.

Suisse.

Portugal.

Italie ("grillo », " cavalletta »).

*Hongrie [XIX ${ }^{\text {ème }}$ siècle].

Roumanie.

Malte.

Japon (" tonosama batta »).

Chine.

Canaries (Iles). 
Locusta danica Linn. (Pachytylus danicus Auriv.; Oedipoda migratoria Burm.).

Pachytylus sp. (I)

Dissosteira carolina Linn

D. longipennis Thomas

Oedipoda miniata Pall. Oe. caerulescens Linn.

Trilophidia cristella Stål

T. annulata Thunb.

Bryodema tuherculatus Fabr.

Sphingonotus azurescens Ramb.

Prionotropis hystrix Germ.

P. flexuosa Serv. (Thrinchus culligera] perezii Bol.).

Ommexych: virens Serv.

O. servillei Blanch.

Aulcrches miliaris Linn.
Eigypte.

Atrique orientale allemande.

Union de 1'Afrique du Sud: Cap. Maurice (Ile).

*Salvador.

Australie: Queens?and occidental et septentrional; districts côtiers de la *Nouvelle-Galles d" Sud ("yellow-winged locust ») ; *Tictoria.

Nouvelle-Zélande.

*Et tats Fédérés Malais ("Malayan locust ").

Etablissements du Détroit.

*Johore.

Canada ' ( Carolina locust »)

Etats-Unis (" long-winged grasshopper », "long-winged locust »).

Autriche: Carso gorizien.

Italie (" cavalletta comune", "cavalletta rossa", "cavalletta celeste ", "grillo rosso ", "grillo celeste ").

Autriche: Carso gorizien; Dalınatie En français, "criquet bleu» Java.

))

Chypre.

Chine septentrionale.

En français, "criquet à larges ailes \%.

Chypre.

Autriche: *Carso gorizien ("konj»). spagne.

Paraguay (" tukú-ihvíh )).

Ceylan.

En anglais, "painted locust», "spotted locust".

(I) C'est le nom que l'on donne, en attendant qu'elle soit identifiée d'une manière plus précise, à la sauterelle nuisible que l'on trouve dans les pays ci-contre.

Nous plaçons ce nom ici, Pachytylus étant, d'après K1RBr, synonime de Locusti. Ajoutons que cet orthoptère a d'abord été déterminé, dans les Etats Fédérés Malais, par $P$. danicus Auriv., synonime de $L$. danica Linn. 
Aularches punctatus Drury (Phymateus punctatus Thunb.).

Zonocerus elegans Thunb.

Z. variegatus Linn.

Atractomorbhu crenulata Fabr.

A. crenaticeps Blanch.

A. psittacina De Haan

Diedronoins discoideus Serv.

Elceochlora trilineata Serv. (Xiphicera trilineata).

$E$. viridicata Serv.

Chromacyis miles Drury (Rhomalea miles Pict. et Sauss.).

C. speciosa Thunb. (C. stolli Brun.).

Zoniopoda tarsata Serv. (Gryllus servillei Guér.).

$Z$. omnicolor Blanch.

Tropidacris collaris Stoll (T. cristata Seudd.).

T. $d u x$ Drury

Oxya velox Fabr.

O. vicina Bruru1.
Ceylan: *districts de Kurunegala, Matale, Kadugannava.

Atrique orientale allemande («bunte Stinkschrecke »).

Mozanbique: Inhambane; Lourenço-Marques.

Union de 1'Afrique du Sud: Transvaa1; Cap.

En anglais, "elegant grasshopper $)$.

Conge belge.

Ceylan.

Java.

Hawaï: îles d'Oahou et de Kaouaï.

Australie: Queensland («long-nosed locust »).

Java.

Uruguay

$\left.\begin{array}{l}\text { Argentine } \\ \text { Uruguay }\end{array}\right\}$

*Argentine: territoires nationaux du Chubut, de Río Negro et de La Pampa; partie nord-ouest de la prov. de Buenos-Aires et la région de San Rafaél dans la prov. de Mendoza ("tucura). Uruguay ("tucura»).

Paraguay.

Nouvelle-Zélande ("black cricket»).

Paraguay.

* Guyane anglaise : district de Bartica.

Uruguay ("langosta quebrachera »). Trinité [Ile de la] ("giant locust »).

*Guyane anglaise: Berbice.

En français, "criquet géant", "sauterelle géante".

*Japon ("inago »).

Ceylan.

Java.

Hawaï : île d'Oahou.

Australie: Queensland ("shorthorned locust ").

*Japon ("kogane inago »). 
Oxya anmulicornis Mats.

Pezotettix sp.

Orthacanthacris agyptia Linn. (Acridium cegyplium Stål).

Cyrtacanthasris nigricornis Burm. (Acridium melanocorne Serv.).

C. rosea De Geer (Acrydium roseum).

C. maculicolits Walk. (Acridium maculicolle).

C. succincta Linn. (Acrydium succinctum Oliv.).

C. septemfasciata Serv.

C. aruginosa Sto!1 (Acridium aruginosum Burm.).

C. guttulosa Walk.

C. exasta Walk.

C. ? proxima Walk.

C. plagiata Walk.

C. luteicornis Serv. (Acridium luteicorne).

Schistocerca columbina Thunb.

S. zapoteca Scudd.

S. australis Siudd.
*Japon (《higemadara inago »).

Autriche: Carso gorizien.

Portugal.

Italie ("locusta egiziana», "grillone $)$.

Autriche: Carso gorizien; Dalmatie. Egypte.

Ceylan.

Java: *résidences de Semarang, de Rembang, etc. ("grote sprinkhaan", "walang kaioe ").

Java.

Australie: Queensland septentriona1; Nouvelle-Galles du Sud à Sydney ("large coast locust )). *Inde ("Bombay locust »).

Congo belge.

Afrique orientale allemande.

*Rhodésia méridionale (" red locust n).

*Mozambique ("gafanhoto de asas vermelhas", "gafanhoto verme1ho"; "nhazombe" l'insecte adulte et "cambezi" l'insecte non ailé, dans la région de Quélimane).

Union de 1'Afrique du Sud : Transvaal; Orange; *Natal; Cap ( red locust ", " new locust", "large locust ", "coast locust", "redwinged locust ", " purple-winged locust ", "egyptian locust"). Java.

Australie: Queensland ("shorthorned locust ").

Australie: *Queensland ; NouvelleGalles du Sud.

Australie: Queensland (" shorthorned locust $)$.

Java.

*Porto-Rico.

Costa-Rica.

Brésil: *Etat de Parahyba do Norte (Campina Grande); *Etat de Santa Catharina (près de la 
Schistocerca tatarica Linn. (Acridium peregrinum Oliv.; A. [Schistocerca] peregrinum Stål; $S$. peregrina Brunn.).
S. paranensis Burm.

S. pallens Thunb. localité appelée Macacos); *Etat de Rio Grande do Sul (Cacéquy). Angleterre.

Portugal.

Espagne (prov. de Cadix).

Gibraltar (environs).

Chypre.

Perse.

*Inde ("migratory locust ).

Canaries (Iles).

*Maroc, Protect. tranç. (« Djeradel-Arbi », en arabe).

*Algérie.

*Tunisie.

Tripolitaine.

*Egypte ("migratory locust»).

*Erythrée.

*Nigéria [?] ("migratory locust »).

*Afrique orientale anglaise ("locust $)$.

*Afrique orientale allemande ("nsige"), en langage Kisuaheli; "gelbe Wanderheuschrecke ", "ägyptische Wanderheuschrecke", en allemand).

Sud-Ouest africain allemand: Namaqualand.

Mexique (?).

En allemand, appelé aussi «afrikanische Wanderheuschrecken "; en français, "criquet pèlerin ", " sauterelle pèlerine ", " criquet nomade ", "le pèlerin ".

*Costa-Rica («langosta», "chapulín»).

Patos (Ile de).

Chacachacare (Ile de).

Monos (Ile de).

*Trinité (Ile de la): Carenage; Icacos.

*Brési1 ("gafanhoto »).

*Bolivie.

*Argentine $\{$ (“langosta »)

*Paraguay

*Uruguay ("langosta migratoria »). En allemand, "südamerikanische Wanderheuschrecke ». 
Schistocerca pallens Thunb.

Jamaïque.

Haïti.

Porto-Rico.

St.-Christophe (St.-Kitts): la seule île, parmi les petites Antilles, où l'insecte apparaît de temps en temps et cause quelques dommages.

Antigua.

Montserrat.

Dominique.

Ste.-Lucie.

St.-Vincent.

Barbade.

Grenade.

*Guyane anglaise.

En espagno], "saltamontes".

S. vicaria Walk. (Acridium vi- Guyane anglaise: Berbice. carium).

S. americana Drury (Acridium [Schistocerca] americanum Stål).

S. perwoviaila Arribálzaga

S. praesignata Rehn in ms.

Schisiocerca sp.

Traulia flavoannulata Stå1

Trigonophymus pratensis Brun.

T. arrogans Stå1 (Dichroplus arrogans Bruin.).

$T$. bergii Stål

Melanoplus affinis Scudd.

$M$. atlanis Riley

M. spretus Thoinas

Mexique: *Yucatan (" langosta »). En allemand, "mittelamerikanische Wanderheuschrecke "; en anglais, "American acridium ", "American locust», "bird grasshopper"; en français, "criquet américain $\%$.

Pérou ( (langosta »).

Trinité (Ile de 1a).

Guyane hollandaise [Surinam].

Java.

Uruguay

Argentine (" tucura »)

Brésil.

Canada: *Colombie britannique. " *Nanitoba et dans 1 "*ouest ("lesser migratory locust»).

*Etats-Unis ("lesser migratory locust ").

En anglais, on l'appelle encore "Atlantic locust"; en français, "petite sauterelle".

Canada: *provinces occidentales ("Rocky Mountain locust»).

*Etats-Unis ("Rocky Mountain 10cust ", "migratory locust ", " hateful grasshopper »). 
Melanoplus spretus Thomas

M. daresoni Scudd.

M. gladstoni Scudd.

11. femur-vubrum De Geet

M. angustipennis Dodge

M. packardii Scudd.

M. minor Scudd.

1I. differentialis Thomas

1I. bivitiatus Say

Podisma salamandya Fisch.

P. schmidtii Fieb.

P. pedestris Linn. (Pezotettix pedestris Fisch.).

P. alpiniti Koll.

Calliptamus italicus Linn. (Calo. ptemus italicus Burm.).
En allemand, " Felsengebirgsheuschrecke"; en français, "sauterelle des Montagnes-Rocheuses". Canada.

* " («red-legged locust »).

*Etats-Unis ( « red-legged grasshopper ", "red-legged locust ").

En français, "sauterelle à cuisses ronges $\%$.

Canada.

$$
\text { " ("Packard's locust ). }
$$

"Etats-Unis ("differential grasshopper ", "differential locust ").

*Canada ("two-striped grasshopper», "two-striped locust »).

* Etats-Unis ("two-lined grasshopper", "two-lined locust", "twostriped locust ", "yellow-striped locust $)$.

Autriche: Carso gorizien. » »

Bulgarie.

En français, "criquet aptère ». Autriche: Basse-Autriche (Hollenstein, Heuberg, Mittenberg près de Mödling); Styrie (*Gratz; Tuffer [domaine de Gairach]) ; *Carniole.

Allemagne.

Suisse.

France: *Sud-Ouest [Charentes]; Château-Gombert aux environs de Marseille ("caloptène italique », "criquet italien ").

Portugal.

Esspagne.

*Italie ("cavalletta italica", "grillastro italiano $)$ ).

Autriche: Val Sugana; *Carso gorizien ("konjič »); Dalmatie.

Hongrie.

*Grèce.

Malte.

Canaries (Iles).

Algérie.

Tripolitaine et Cyrénaïque.

Egypte. 



\section{BIOLOGIE E'T MGURS DES SAUTERELLES}

Quoique la biologie et les mœurs des sauterelles aient été l'objet des recherches et des études d'un grand nombre d'auteurs, elles présentent néanmoins encore des points obscurs. On comprendra facilement combien cela doit nuire à l'établissement d'une organisation plus efficace de la lutte contre ces orthoptères.

Nous résumerons ici tout ce qu'il nous a été possible de puiser à ce sujet dans les documents mis à notre disposition, en groupant, pour chaque espèce de sauterelles, les renseignements provenant des divers pays.

\section{DONNÉES SUR LE CYCLE ÉVOLUTIF DES DIFFÉRENTES ESPÈCES}

En exposant les renseignements qui suivent, nous nous en tenons à la nomenclature et à l'ordre de succession des espèces déjà adoptés pour le chapitre précédent.

Dociostaurus maroccanus. - Au Portuga1, les adultes de cette espèce ont été observés en juillet. En Espagne, l'époque de l'apparition de l'ailé est variable, comme le sont les conditions des différentes régions qu'il envahit ordinairement; ainsi dans la province de MIalaga, les adultes volent pendant le mois de juin, tandis que dans la partie centrale de la péninsule, on ne rencontre cet insecte à l'état parfait qu'au mois de juillet suivant. En I t a lie, le criquet marocain, est, des espèces les plus nuisibles, celle qui se montre la première munie de ses ailes; cependant, on trouve difficilement des ailés avant le mois de juin ; ce n'est qu'exceptionnellement qu'on peut en rencontrer en Sicile au mois de mai, et c'est vers la fin d'août que l'on voit les derniers; l'évolution est plus précoce ou plus tardive selon l'altitude des lieux de naissance, leur situation et les conditions atmosphériques qui accompagnent cette évolution. En Autriche, les adultes de cette espèce font leur apparition de juillet à août. En Hongrie, les premiers ailés du criquet marocain se rencontrent entre le 5 et le ro juin; plus tard, leur nombre s'accroît ; entre le 20 et le 25 juin, presque tous sont munis de leurs ailes, bien qu'il arrive parfois de trouver des insectes encore non ailés même vers la fin de juin et dans la première moitié de juillet. En B ulgarie, D. maroccanus devient ailé dans la seconde moitié de mai. En Grèce, c'est en été qu'il arrive à son évolution complète. L'époque de l'apparition des adultes dans l'Afrique du. Nord est assez variable. 
En Algérie, les ailés commencent à se montrer ordinairement vers la moitié du mois de mai. Des adultes auraient même été observés dans les premiers jours du mois d'octobre suivant.

En I t a 1ie, si le temps est beau et chaud, D. maroccanus s'apprête à la reproduction une semaine environ après la dernière mue ; 1'accouplement dure plusieurs heures. Det1x ou trois mâles se tiennent ordinairement auprès de la femelle. La copulation paraît se répéter trois fois; elle a lieu sûrement deux fois. En Autriche, l'accouplement se produit en plein été. D'après les observations recueillies en Hongrie, il commence parfois dans ce pays immédiatement après la métamorphose, mais, en général, il n'a lieu que de I 5 à 25 jours plus tard, c'est-à-dire dans la première moitié de juillet environ et il se répète une ou deux fois après chaque ponte et même plusieurs fois durant les périodes de forte multiplication. Dans l'Afrique du Nord également, d'après les observations de Kürckel D'Herculais pour l'Algérie, l'accouplement, comme la ponte, a lieu plusieurs fois après l'apparition des adultes; il commence le plus souvent vers le I5 mai.

Dans les différents pays, la femelle dépose de préférence ses œufs dans le sol à des endroits déterminés : en général dans des terrains élevés, rocailleux, très sees et compacts, sans végétation et, ordinairement, incultes, bien que la ponte puisse avoir lien aussi, exceptionnellement, dans des lieux cultivés. Ėn Grèce, les femelles utiliseraient même les terrains qui conservent un certain degré de fraîcheur et assez meubles.

Lorsqu'elle est sur le point de pondre, la femelle creuse d'abord un trou dans le sol avec son abdomen qu'elle a rendu rigide et auquel elle imprime des mouvements ondulatoires qui rappellent ceux d'une sonde; elle y dépose ensuite ses œufs, dont le nombre est variable, en les disposant en plusieurs séries. En exécutant cette opération, la femelle enduit les parois du trou d'une substance agglutinante, sécrétée par des glandes annexées à l'appareil génital ; la terre se trouve ainsi cimentée de manière à former autour des œufs une espèce d'étui (coque ovigère ou oothèque), en forme de massue, un peu recourbé vers le milieu, muni, vers le haut, d'un opercule rond et concave, formé lui aussi de terre agglutinée. Les oothèques sont plantées à une profondeur de 3 à $4 \mathrm{~cm}$ et le terrain qui les renferme est littéralement criblé de trous.

Ein I talie, la ponte paraît s'effectuer en deux ou trois fois. En Hongrie, cette opération commence ordinairement dans la première décade de juillet et, dans les années favorables à une multiplication intense, elle se répéte une ou deux fois par périodes de trois à quatre semaines, c'est-àdire même en août et en septembre; on observa, en effet, en I889, que le criquet marocain était encore occupé à pondre le I7 septembre. En Bu1garie, la femelle pond vers la fin de juin et pendant les mois de juillet et d'août. Pendant ces deux derniers mois, le même phénomène s'accomplit en Grèce, où d'habitude il n'y a qu'une seule ponte par an, mais on dit qu'il peut y en avoir deux et même plusieurs ; cela dépend de différentes causes, surtout des conditions climatiques. Dans l'Afrique du Nord, les pontes, comme on l'a observé en Algérie, ont lieu principalement 
dans la seconde moitié de juin, mais elles se prolongent jusqu'an mois de juillet.

Il résulte des observations faites en Espagne que, lorsque les femelles ont été fécondées, les mâles meurent après les avoir aidées aux opérations de la ponte. Le nombre moyen d'œufs contenus dans chaque oothèque (" canuto ", " canutillo ") est de 26 . En I ta 1 ie, on compte dans chaque oothèque de 20 à 25 œufs. Par contre, les coques ovigères ramassées en Autriche contiennent en moyenne de 35 à 40 aufs. En Grèce, on est incliné à croire que chaque femelle pond en moyenne 32 ceufs. Dans 1'Afrique du Nord, chaque oothèque contient de 30 à 40 oufs.

Au Portuga1, on a observé les œufs éclore du I2 mars au 4 avril. L'époque où les larves ( (mosquitos») apparaissent en Es pagne est assez variable. Ainsi dans la région méridionale de ce pays, elles éclosent, certaines années, dans la première quinzaine de mars; dans les provinces de Malaga, Almeria et Cadix à la fin dudit mois ; à Huelva, à Cordoue et dans la région centrale dès la fin d'avril ; il en est de même dans la province de Madrid; dans la province d'Avila, elles naissent en mai. Si, dans les régions chaudes de 1'Italie et dans les localités exposées au midi, on brise une oothèque vers la moitié de mars, on voit que les oufs y sont de couleur jaunâtre au lieu d'être blancs, qu'ils ont légèrement grossi et l'on aperçoit fort bien à travers leur coque les formes de la larve. La naissance a lieu à partir de la seconde décade de mars selon la température. M. LunARdoni, Commissaire général pour la lutte contre les sauterelles en Sicile, a trouvé, le 27 mars I9I2, des sauterelles âgées de 5 à Io jours à $800 \mathrm{~m}$ environ au-dessus du niveau de la mer, à Petralia Sottana. Ein IgI4, les premières naissances générales ont été observées le 5 avril, tandis qu'en IgI5 les premières larves n'ont fait leur apparition qu'entre le I $^{\text {er }}$ et 2 mai. Les larves résistent comme les œufs à de basses températures; la nuit du I5 au I6 avril I9I3, il tomba une assez grande quantité de neige dans le territoire de Gangi (Sicile) et le thermomètre descendit au-dessous de zéro; mais les larves ne moururent pas, bien qu'elles fussent à peine écloses. Dans cet état, elles sont de couleur paille clair sale, mais après deux ou trois jours elles deviennent presque noires, puis de couleur marron avec diverses gradations sur les différentes parties du corps. Les œufs pondus dans un endroit donné n'éclosent pas tous dans la même journée, ils peuvent éclore aussi à des intervalles de plusieurs jours ; mais ceux qui sont contenus dans une même coque ovigère éclosent ensem ble. En A u tri c he, les larves naissent de la fin d'avril à la fin de mai. En Hongrie, où la naissance peut commencer dès les derniers jours d'avril, elle a lieu le plus souvent en mai et 1'on rencontre même de jeunes larves à peine sorties de terre jusqu'aux premiers jours de juin; ces dernières proviennent probablement d'œufs pondus en août ou en septembre. Dans les années où la multiplication est moins abondante, la réitération de 1'accouplement, de la ponte et, par conséquent, de 1'apparition des larves n'est pas très évidente; dans ce cas, elle a été, en effet, rarement observée. Mais ce qui est certain, c'est que ces années-là les larves n'apparaissent pas avant la seconde quinzaine de mai et même plus tard. Elles 
naissent aussi en avril et en mai en B ulg a r i e. En G r è c e, l'éclosion commence en février et se poursuit quelquefois jusqu’en mai ; cela dépend de l'exposition de l'endroit où se trouvent les pontes et des conditions climatiques plus ou moins favorables. On a observé également qu'un certain degré d'humidité du sol est absolument nécessaire pour que l'éclosion des larves se produise facilement; des œufs conservés pendant trois ans dans un milieu sec, ne sont pas éclos, tout en ne perdant rien de leur vitalité. D'autre part, une humidité excessive, comme l'inondation des lieux de ponte, empêche ou retarde l'éclosion. Si les eaux submergent le sol pendant plusieurs jours, trois au minimum, la vitalité des œufs est détruite, tandis que les pluies, quelque abondantes qu'elles soient, si le sol les laisse s'écouler facilement, n'ont aucune influence nuisible sur les oufs et sur les jeunes insectes. Nous rappellerons à ce propos que d'après des observations effectuées en Italie (LUNARDoNI), les œufs renfermés dans les oothèques résisteraient à la submersion pendant des mois et des mois sans en souffrir. La sécheresse, comme on l'a observé en Grèce, n’est pas contraire non plus, en général, à la vitalité des œufs. Mais si durant les trois premières semaines qui suivent la ponte il règne une grande sécheresse, les œeufs se rident et perdent leur pouvoir germinatif, tandis qu'après la troisième semaine ils n'ont plus rien à craindre à cet égard. Dans l'Afrique du Nord les larves naissent le plus souvent 9 ou Io mois après la ponte. En particulier, en Algérie, l'éclosion commence déjà dans les derniers jours de mars; elle atteint son maximum vers la moitié d'avril et se prolonge jusqu'en mai.

D'après ce que l'on a constaté dans les pays où vit cette sauterelle, c'est après la troisième mue que les rudiments des ailes (moignons alaires) apparaissent sur le corps de la larve (I).

Après avoir subi cinq mues dans un espace de temps plus ou moins variable - en Algérie, par exemple, comme nous l'avons déjà dit, l'éclosion commence vers la fin de mars et 1'on voit déjà des adultes à la moitié de mai - l'insecte dévient ailé et peu de temps après il est apte à entreprendre même de longs vols.

Chortoicetes pusilla. - Dans les districts occidentaux de la Nouvelle-Galles du Sud (Australie) on a observé que cette espèce pondait ses œufs dans les plaines à terre rouge durant le mois de novembre et qu'une seconde ponte avait lieu un mois après. Chaque ponte ne comprend que I9 oufs.

Un fait curieux, c'est que lorsqu'une femelle s'apprête à pondre, un bon nombre de mâles prennent place autour d'elle, alors que deux d'entre eux se tiennent plus rapprochés de la pondeuse.

Les larves apparaissent du mois d'août au commencement de septembre.

(r) En Eispagne, où l'insecte, dès la première mue, porte le nom rulgaire de "mosca", l'orthoptère prend à ce stadie le nom de "saltón". 
Chortoicetes terminifera. - Dans la Nouvelle-Galles du Sud, où cette sauterelle peut avoir, paraît-il, jusqu'à trois générations par an, les adultes font leur apparition vers la fin d'octobre et en novembre, vers la fin de décembre et en janvier et encore vers les mois de mars et d'avril.

Les femelles choisissent pour la ponte des étendues de terrain relativement limitées, quelquefois cultivées, mais souvent sur des points dénués de végétation, dans des pâturages, sur les bords des routes, des sentiers battus par le bétail, etc.

Les œufs, au nombre de 20 ou davantage, sont pondus à une profondeur de $21 / 2$ à $71 / 2 \mathrm{~cm}$, dans des trous creusés par la femelle ; ils sont complètement enveloppés dans une sécrétion crémeuse, qui en se solidifiant forme une espèce d'étui protecteur ayant un aspect spongieux cartacé.

Les œufs pondus par les femelles apparues en mars et en avril semblent traverser les mois d'hiver sans éclore ; ceux qui ont été pondus pendant les mois d'été éclosent dans l'espace d'environ trois semaines.

La première apparition de larves a lieu aux mois d'août et de septembre et au commencement d'octobre, la deuxième au mois de novembre et de décembre, 1a troisième vers les mois de février et de mars.

L'insecte subit une série de mues. La période qui comprend les trois premiers stades d'évolution est d'environ trois semaines. Le stade suivant dure environ dix jours et celui qui vient après est de deux à trois semaines. Ces deux derniers stades sont marqués par l'apparition des rudiments des ailes. Le stade suivant est celui où l'insecte devient ailé.

Les adultes vivent pendant une période d'environ six semaines et peuvent pondre plusieurs groupes d'œufs. Comme les stades qui précèdent l'état adulte occupent un espace d'environ sept semaines, la durée totale de l'évolution de l'insecte, à partir de l'éclosion des œufs, est de treize semaines.

Oedaleus senegalensis. - D'après ce que l'on a observé dans la Nouve11e-Galles du Sud, dans le district de Singleton (mars 1907), cette espèce pond ses œufs au sommet de petites élévations de terrain, découvertes, herbeuses, de consistance dure, argileuse. Les oufs, au nombre de 30 à 50 , sont protégés par un revêtement et placés dans des trous à une profondeur de $3 \mathrm{~cm}$ environ.

Locusta pardalina. - Dans le Sud-Ouest africain a11emand, les adultes apparaissent principalement de février à mai. Dans l'Union de 1'Afrique du Sud les adultes envahisseurs se montrent en avri1.

Dans le premier de ces deux pays la ponte a lieu du mois de mai au mois d'août et dans l'Uni on pendant les mois d'hiver; elle peut se prolonger parfois de mai à aô̂t comme dans le Sud-Ouest africain allem a nd.

Les œufs éclosent après la première grande pluie d'été ( $\mathrm{Sud}$-O u est africain allemand); d'après ce qui a été observé dans le territoire de 1'Trnion, la ponte est favorisée par deux facteurs: une quantité suffi- 
sante de chaleur et une quantité suffisante d'humidité. Cette dernière, quand la chaleur manque, exerce sur les oufs une action destructrice; par contre, en 1'absence d'humidité, les œufs conservent leur vitalité dans le sol pendant plusieurs années. Ordinairement les œufs éclosent en dix ou douze jours, après qu'ils ont été suffisamment humectés. Comme les pluies abondantes tombent généralement en septembre, l'éclosion commence d'ordinaire vers la première semaine d'octobre et l'insecte devient adulte dès la moitié ou la fin de décembre. Le stade d'œuf peut donc durer un mois ou plusieurs années. La longueur du stade aptère dépend de la quantité de nourriture disponible; dans des conditions favorables, il peut ne pas durer plus de soixante jours, mais dans des circonstances défavorables il peut se prolonger pendant une centaine de jours ou davantage.

Locusta australis.-D'après les observations effectuées dans 1'Etat de Victoria (Australie), la femelle pond, dans des trous creusés dans le sol, de 50 à 80 œufs et même davantage, renfermés dans un étui d'environ $2 \frac{1 / 2}{\mathrm{~cm}}$ de long et protégés par une sécrétion blanche, spumeuse.

L'opération de la ponte dure environ six heures et les lieux préférés sont ceux en pente douce, sans végétation ou en ayant fort peu, mais aussi les sentiers battus par le bétail, les routes, les plaines, etc.

Il y a probablement deux générations par an.

Les œufs pondus en octobre ou en novembre, étant donnée la chaleur de l'été, éclosent en un mois, tandis que ceux pondus en mars hivernent et éclosent vers le mois de septembre.

En plein air, trois mois environ après l'éclosion, la sauterelle est devenue complètement adulte; en captivité, d'après les expériences de FrENch, elle ne met que deux mois pour atteindre ce stade.

Locusta migratoria. - Les adultes font leur apparition en Roumanie au commencement de juillet; ils s'accouplent en août, à peu près entre le I 6 et le 29 , et à partir de la fin du même mois, la ponte commence. Les larves naissent de la fin d'avril aux premier jours de mai; vers les dix derniers jours de juin l'insecte s'apprête à subir sa métamorphose. La température peut naturellement produire des variations dans les différentes périodes d'évolution de cette espèce.

Pachytylus sp. - C'est ainsi que 1'on a dénommé provisoirement, en attendant qu'elle soit exactement identifiée, l'espéce qui infeste les Etats Fédérés Malais, les Etablissements du Détroit, le J ohore, etc., et que l'on croyait être d'abord P. danicus Auriv. $=$ Locusta danica Linn.

L'insecte, d'après ce qui a été observé dans les Etats Fédérés Málais, présente une très grande variabilité de coloration aux divers stades de son évolution. Enn général, à l'époque de la reproduction, les mâles sont de couleur jaune soufre luisant et les femelles ont une teinte brun sale mat.

En captivité, les adultes obtenus d'œufs sont restés en vie, dans un 
cas pendant soixante-dix jours, et dans un autre pendant soixante jours seulement. Dans le premier cas, l'accouplement a été constaté dix-neuf jours après la dernière mue, dans le second après dix-sept jours.

La ponte se fait d'une manière éparse, car il est rare qu'une bande considérable puisse trouver une surface de terrain continue assez étendue pour y pondre ses œufs, à moins que ce ne soit un terrain sur lequel on a brûlé récemment la couverture de "lalang» (Imperata arundinacea) ou appartenant à une zone minière abandonnée.

Les terrains favorables à $l_{a}$ ponte que les sauterelles ont çà et là à leur disposition sont les terre-pleins des lignes de chemins de fer, les bords des routes, le "Kampong land \#-terrain entourant un village malais et consistant en général en un sol herbenx sur lequel sont cultivés des arbres fruitiers - les champs de riz ("padi sawahs »), les endroits récemment déboisés, les ravins peu profonds, les clairières dans la jungle ("Sakaï clearings », du nom de certaines tribus sauvages de la péninsule de Malacca).

On n'a pas observé qu'il y ait une époque déterminée pour la reproduction : œufs, larves à tous les stades et adultes se trouvent souvent dans un seul district à la même époque. S'il s'agit d'adultes, les individus qui composent une bande peuvent appartenir à tous les degrés de la maturité : certains viennent d'acquérir les ailes, d'autres s'apprêtent déjà à pondre. Mais en général toutes les femelles d'une même bande pondent dans l'espace de dix jours ; la première ponte a lieu ordinairement le jour qui suit celui où la bande s'est posée sur le terrain favorable à cette opération; c'est le second jour que les sauterelles pondent le plus grand nombre d'œufs ; elles choisissent généralement un terrain plutôt meuble, bien que l'on ait observé fréquemment que la ponte se faisait dans des terrains lourds argileux, même lorsque des terrains plus légers et plus convenables se trouvaient dans le voisinage; les terres défrichées depuis peu sont très recherchées.

Pour la ponte, la femelle creuse un trou dans le sol, à une profondeur qui varie de $21 / 2$ à $71 / 2 \mathrm{~cm}$ et davantage; elle pond dans chaque trou de I5 à 60 œufs qu'elle protège en les enveloppant chacun, puis tous ensemble, d'une substance écumeuse 1uisante. Dans des conditions ordinaires, les larves commencent à apparaître treize ou quatorze jours après la ponte.

Ces jeunes larves passent par cinq phases successives d'évolution: la durée moyenne du premier stade est de quatre à cinq jours; celle du second et du troisième de cinq à six jours; le quatrième dure de six à sept jours; le cinquième de sir à huit jours.

Si l'insecte est élevé en captivité, dans des conditions se rapprochant le plus possible des conditions naturelles, la dernière mue, c'est-à-dire celle après laquelle l'insecte devient parfait, a lieu de vingt-quatre à quarantesept jours après 1'éclosion ; de vingt-six à trente-deux jours si l'insecte est en liberté.

Dissosteira longipennis. - D'après ce qui a été observé pendant l'été de IgI3 au Nouveau-Mexique (Ettats-Unis d'A méri$q u e)$, les adultes y ont fait leur apparition en juin. Toutefois on rencon- 
trait encore des individus non ailés de cette espèce vers la première moitié de juillet.

Le plus souvent les œufs sont pondus en sol sablonneux, quelquefois dans un terrain dur, de la fin d'août au commencement de septembre; il ne paraît y avoir qu'une seule génération par an.

L'éclosion commence vers la fin d'avril et c'est vers les premiers jours de mai qu'elle est le plus abondante.

Tropidacris dux. - Au sujet de la biologie de cette espèce on a observé que, dans l'île de la 'Trin ité, les adultes se montrent vers la fin de l'année (octobre-décembre).

Les jeunes larves se rencontrent dans les champs au commencement de la saison des pluies (mai ou juin).

Des exemplaires tenus en captivité ont fourni des adultes qui ont pondu. On a constaté que cette sauterelle reste à l'état non ailé de mai à juillet; les femelles pondent en octobre.

L'insecte n'a qu'une génération par an.

Oxya velox. - Au J a pon, l'époque de l'apparition des adultes dépend strictement des conditions climatiques et de l'état plus ou moins avancé de la végétation dans la région infestée; en tout cas, c'est dans la seconde et la troisième décade d'août que les adultes arrivent au maximum de leur développement.

L'accouplement a lieu une ou deux semaines après que l'insecte est muni de ses ailes et, en général, de midi à quatre heures de l'après-midi.

Deux ou trois semaines après qu'elle a atteint l'état parfait, la femelle pond ses œufs. Dans le Japon proprement dit, la ponte a lieu en septembre ou en octobre. La femelle, se servant de son abdomen, creuse dans le sol un trou dont la profondeur varie de 4 -5 "bu " ( $22-\mathrm{I}_{5} \mathrm{~mm}$ ) à I "sun » (uı peu plus de $3 \mathrm{~cm}$ ). Chaque paquet d'œufs pondu dans cette cavité comprend, au minimum, de 20 à 30 œufs, au maximum de 70 à 80 et même Ioo.

L'insecte passe l'hiver à l'état d'œuf.

Les larves naissent entre les mois de mai et de juin de l'année suivante ; toutefois, cela dépend des conditions climatologiques: les larves les plus précoces naissent dans la seconde ou la troisième décade de mai et les plus retardataires apparaissent au mois de juin.

L'espèce en question subit cinq mues avant d'arriver à l'état ailé. La première de ces mues a lieu dix jours environ après l'éclosion de la larre, les autres durent une semaine chacune.

L'insecte meurt après la ponte, en automne, au moment où les gelées blanches commencent.

Cyrtacanthacris sucincta. - C'est au mois d'octobre que les adultes de cette espèce apparaissent dans l'I $n d e$; cette sauterelle vit à l'état ailé d'octobre à juin; sa couleur est d'abord brune avec des raỵures claires, elle ressemble à celle de 1'herbe sèche et protège l'insecte au milieu 
des hautes herbes desséchées où il vit. Les insectes se réunissent ensuite en bandes et sont alors d'un rouge vif, qui persiste pendant quatre mois environ. Durant ce laps de temps, les sauterelles vivent en bandes immenses dans les forêts jusqu'en avril et mai, époque où les individus se dispersent.

L'accouplement commence avec les pluies. La sauterelle prend alors une couleur plus foncée, le rouge cède la place au noir et au brun. Cette coloration sert à cacher l'insecte lorsqu'il se trouve sur l'herbe mouillée ou sur le terrain où il pond ses œufs.

La ponte se fait en juin ou juillet à l'approche des premières grandes pluies. Elle n'a lieu que dans les endroits humides; la femelle pond ses œufs, dans le sol humecté, sous forme d'un petit tas qui en contient à peu près cent.

Les larves éclosent au bout d'environ six semaines. Les jeunes insectes sont verts et. ils complètent leur développement en deux mois environ. L'évolution complète de la sauterelle dure un an. Les jeunes individus se trouvent épars au milieu des longues herbes vertes pendant les mois de pluie. En octobre on voit les premières bandes se diriger vers les terres cultivées pour se nourrir: elles rentrent ensuite dans les forêts.

Cyrtäanthacris septemfasciata. - Lorsque les conditions climatiques sont normales, les ailés apparaissent dans la Province de Mozambique vers la fin d'avril et au commencement de juillet, tandis que dans 1'Union de 1'A friqueduSud on ne les rencontre qu'entre les mois de juillet et de septembre.

Au sud de la Province de Mozambique, l'accouplement se fait entre septembre et octobre; dans le nord, il a lien environ un mois avant. Pendant la période de l'accouplement, les ailes inférieures de l'insecte, habituellement incolores, présentent à leur base une coloration rougevin. Dans l'Union l'accouplement se produit en septembre ou en octobre.

Dans la Province de Mozambique, la ponte a lieu quelques jours après l'accouplement. La femelle choisit les terrains consistants ; elle préfère ordinairement les endroits découverts ou cultivés, et elle évite toujours ceux qui sont sujets à être submergés par les pluies ou par les inondations. I a ponte a lien, d'habitude, le matin de bonne heure. Selon l'importance de la bande,l'opération dure plus ou moins (de une à cinq semaines) indépendamment des pluies, mais elle se fait ordinairement tout de suite après les premières pluies. Chaque femelle pond en moyenne go œufs renfermés dans une oothèque placée dans le sol à une profondeur de 2 ou 3 centimètres. Les mâles, qui vivent plus longtemps, peuvent s'accoupler avec plusieurs femelles; celles-ci meurent presque immédiatement après la ponte. Lorsque des circonstances particulières empêchent de reconnaître les foyers de ponte au moyen des trous de ponte, la présence de ces femelles mortes indique aux personnes expérimentées l'existence de ces foyers. Dans 1'Union, les œufs'sont pondus en octobre, novembre et décembre, mais ordinairement en novembre.

D'après ce qu'on rapporte pour la Province de IIozambique, 
la vitalité des œufs de cette espèce n'est pas très grande; un excès de sécheresse ou d'humidité les détruit en pen de temps.

La durée de l'incubation varie de trois à six semaines et elle est très influencée par la température; dans la région du Zambèze, elle ne dure que trois semaines. Dans l'U n i on, la période d'incubation est approximativement de trente jours.

Dans de bonnes conditions de lumière et de chaleur, le jeune insecte subit cinq mues en sept ou huit semaines (Province de Moza mbique). Dans l'Union, douze semaines environ s'écoulent de l'état de larve à la métamorphose finale; le stade d'adulte dure environ huit mois. Les jeunes crthoptères sont d'abord cendrés, avec des taches jaune clair qui augmentent après chaque mue, de sorte que l'insecte finit par être d'une couleur jaune-paille.

Schistocerca tatarica. - Les adultes apparaissent dans l'Inde deux fois par an: de la moitié de novembre à la fin de février et de la moitié de mai à la fin d'août. Dans le Protectorat français au MI a roc, les premiers vols furent signalés, en IgI4, le 20 novembre, au sud d'Agadir. Quoique les adultes se soient montrés parfois exceptionnellement en Algérie pendant l'hiver ('Tell, littoral oranais), ils y apparaissent le plus souvent en avril et en mai. En Tunisie où 1'on en a vu, par exemple, en Igo7 et en Igo8, au mois de janvier, dans les territoire militaires - on ne les rencontre normalement que trois ou quatre mois plus tard. En E r y th r é e, la période d'apparition des adultes rarie entre janvier et février dans la plaine et dans la zone intermédiaire entre la plaine et le haut-plateau. Sur ce deruier, les apparitions peuvent avoir lieu entre mai et juin, si les insectes viennent des régions limitrophes. Il est probable que la sauterelle qui a surtout endommagé la Nigéria septentrionale et méridionale est le $S$. tatarica. Si on 1'admet, il y aurait fait, semble-t-il, son apparition vers le mois de janvier ou de cette date jusqu'en avril. Il résulte des renseignements que l'on possède sur les visites des sauterelles dans la Nigéria méridionale que ces insectes s'y seraient montrés précisément vers le mois d'avril. L'apparition des ailés dans l'Afrique orientale anglaise a été observée à différentes époques de l'année, mais plus fréquemment de février à arril et puis en novembre. C'est principalement à l'époque des petites pluies (de novembre jusqu'en février), mais aussi à celle des grandes pluies (mai et juillet) que les bandes ailées font leur apparition dans 1'A frique orientale allemande.

D'après les observations faites dans les Colonies françaises de l'Afrique du Nord, les sauterelles prennent pendant l'hirer une coloration rouge carmin.

On a remarqué dans 1'Inde que, partout où une bande se pose pendant plus de six heures, l'accouplement se produit, et que ce dernier, à son tour, dure environ quatre heures. A mesure que les individus de cette espèce, en venant de l'intérieur de 1'Afrique, avancent vers le 11ord de ce continent, ils font des étapes de quatre ou huit jours, pendant lesquelles 
ils s'accouplent. L'accouplement se répète, semble-t-i1, plusieurs fois, à des intervalles de quinze ou vingt jours. Dans la plaine de 1'Erythrée, il y a des accouplements en novembre et décembre, peut-être même en février; sur le haut-platean ils ont lieu toute l'année sauf en novembre, décembre, janvier et février.

D'après ce qu'on reporte sur l'Afrique orientale allemande, les femelles sont déjà fécondées lorsqu'elles arrivent dans cette région.

A l'époque de l'accouplement, les sauterelles prennent une couleur jaune citron, qu'elles conservent ordinairement durant la période de la ponte ( $\mathrm{nde}$, Col. franç. de 1'A frique du Nord); au contraire, dans la Nigéria, durant cette dernière période, la coloration serait brune.

Les femelles choisissent surtout pour la ponte, dans 1'Inde, les parties sableuses de terrains plutôt secs et meubles, ou bien des terrains meubles, légers mais en même temps frais - lits et berges sablonneuses d'oueds - dans les Colonies françaises de 1'Afrique du Nord, bien qu'elles puissent $y$ utiliser aussi les terrains compacts et exceptionnellement aussi les zones cultivées.

Les femelles portant leurs mâles sur le dos crensent à l'aide de leur abdomen des trous et y pondent, à une profondeur de 5 à $8 \mathrm{~cm}$, les œeufs qu'elles rétnissent en amas, en grappes (grappes ovigères) d'une longueur de 3 à $4 \mathrm{~cm}$; lorsque 1'opération est terminée, elles les recouvrent d'une matière écumeuse qui forme comme un bouchon sur le trou de ponte. Chaque femelle peut donner, après des accouplements répétés à des intervalles de quinze ou vingt jours, de quatre à six pontes successives (Col. franç. de 1'Afrique du Nord). Chaque ponte se compose de 5o, 80, 90 et même roo wufs, de couleur chocolat, qui devient plus foncée vers les pôles (Inde) ou d'abord d'un jaune d'or, puis gris violacé (Col. franç. de 1'Afrique du Nord). Les différents trous de ponte sont très rapprochés les uns des autres, de sorte que la sturface du sol qu'ils occupent et qui, dans 1' I n d e comme dans les Colonies françaises de l'A f r i que du Nord, peut avoir pour un même vol une étendue de 3-4 ha, parait absolument criblée de trous, ce qui donne une idée de la quantité énorme d'œufs qui y sont déposés.

La ponte a lieu immédiatement après 1'accouplement ou peu de temps après. Dans 1'In de, elle a lieu plus précisément, dans le Radjpoutana, an commencement des pluies du sud-ouest en juin ou juillet et de nouveau, si les conditions sont favorables, vers octobre; dans la zone de la frontière du nord-ouest, elle se fait généralement en mars on en avril et de nouveau, si les circonstances le permettent, vers le mois d'août. Dans la Nigéria, la ponte paraît aussi avoir lieu au commencement des pluies, en mars ou avril; toutefois on dit que lors d'une apparition de sauterelles à Yola (Nigéria septentrionale) la ponte se fit en janvier, après quoi la bande envahissante disparut. Il n'y aurait, paraît-il, qu'une seule génération par an dans toute la Nigéria. Dans l'Afrique orientale anglaise, la ponte a lieu - à Voi, par exemple - à la fin de novembre.

Dans 1'Inde, les S. tatarica restent dansle stade d'œuf pendant environ six à sept semaines, après quoi les larves naissent; à peine écloses, elles sont 
de couleur jaune crème clair, puis après qu'elles ont été exposées quatre ou six heures à la lunière, elles deviennent d'un brun jaunâtre. La durée de l'incubation est variable, dans les Colonies françaises de 1'A f r i que $\mathrm{d} u$ Nord, selon les conditions de milieu plus ou moins favorables. Les œufs peuvent commencer à éclore douze on quatorze jours après la ponte, comme aussi après quarante et même soixante-dix jours : ce dernier cas a été constaté, par exemple, pour les pontes faites par les sauterelles apparues, comme nous l'avons déjà mentionné, sur le littoral oranais, plus précisément pendant l'hiver 1895-I896. En moyenne, toutefois, l'éclosion commence entre le vingtième et le trentième jour ; elle n'a pas lieu en même temps pour tous les oufs d'une même ponte, mais elle se produit d'ordinaire successivement pendant quatre, cinq et quelquefois dix jours. En Erythrée, l'éclosion des œufs varie généralement de vingt-et-un à trente jours, à partir de l'époque de la ponte, selon les conditions météorologiques. Pour la Nigéria on n'a pas de données certaines sur la période d'incubation des oufs et l'éclosion des larves. On sait cependant que dans la partie septentrionale du pays (à Yola) on a vu vers le mois de mai éclore des larves d'œufs pondus en janvier. Dans l'Afrique orientale anglaise, les jeunes insectes apparaissent trois semaines environ après la ponte; ils prennentimmédiatement tune coloration foncée. Le stade embryonnaire dure à peut près le même temps (I6-I8 jours) dans 1'Afrique orientale allemande.

Dans l'Inde, la première mue se produit cinq ou six jours après l'apparition des larves et pendant les cinq semaines suivantes ou à peu près l'insecte passe par cinq ou six autres mues; à la dernière, l'ailé apparaît et s'envole après quelque temps, il est alors d'une couleur rosée et il vit environ trois mois, après être devenu jaune au bout de deux mois environ. Dans les Colonies françaises de l'Afrique du Nord, les sauterelles subissent cinq mues à quelques jours de distance l'une de l'autre (à peu près tous les huit jours), après quoi elles sont prêtes pour la métamorphose. Lorsqu'elles viennent de naître, elles sont d'un blanc verdâtre, puis brunes, ensuite noires et plus tard noires avec des dessins jaunâtres ; après la seconde mue vient la couleur rose qui ne change pas même après la troisième ; après la quatrième mue, elles sont d'une couleur rose très vif, qui passe au jaune à la veille de la cinquième mue; la santerelle reprend sa couleur rose au moment de la métamorphose et les mâles la conservent même quelques heures après. Peu après la métamorphose, les femelles sont couleur carmin, les mâles deviennent gris et les teintes jaunes commencent à se montrer ; puis les femelles prennent une teinte d'un gris violacé.

L'insecte devient généralement adulte entre le trente-septième et le quarantième jour après la naissance. La durée complète du cycle d'érolution, c'est-à-dire de l'œuf à l'insecte parfait, est difficile à établir pour la Nigéria, car les renseignements dont on dispose à ce sujet sont plutôt vagues. Il est probable qu'elle varie de soixante à soixante-dix jours. Au moment de se réunir en bandes, les adultes sont de coulentr rouge vif. En Erythrée, l'évolution de l'insecte dure également, en moyenne, une soixantaine de jours, après quoi il est prêt à voler.

Six jours environ après leur apparition, les insectes ont déjà subi 
leur seconde mue dans l' Afrique orientale anglaise; alors, ils sont noirs avec des marques blanches; après tune semaine environ, une autre mue a lieu et l'insecte devient graduellement brunâtre avec des marques jaunâtres. La quatrième mue se fait après sept autres jours et à cette époque les rudiments des ailes apparaissent. L'insecte subit une nouvelle mue dans les dix jours et enfin, après la mue suivante, il devient adulte au bout de deux semaines environ. On peut donc dire qu'après quarante-cinq jours environ de sa sortie de l'œuf, l'insecte est prêt à voler.

Le développement postembryonnaire des sauterelles dans les steppes de l'Afrique orientale allemande dure environ cinquante jours; sur les montagnes de soixante à soixante-dix jours.

Schistocerca paranensis. - Nous disposons de données biologiques sur cette espèce pour Costa-Rica, pour l'île de la Trinité, pour l'Argentine, le Paraguay et l'Uruguay.

Il résulte des observations faites par ALFARo à Costa-Rica, en I915, que les sauterelles à l'état adulte y apparurent dans les premiers jours de juin. Dans la coloration du corps de ces sauterelles, c'est en général le jaune bronzé qui domine, les ailes sont tachetées de brun; toutefois, les exemplaires arrivés à Costa-Rica par le Zarcero étaient tous d'un jaune pâle et paraissaient plus petits que ceux d'Orotina et du Guanacaste. Enn mai ou juin 1885 , des adu1tes de cet orthoptère arrivèrent à Icacos, à l'extrémité sud-est de la Trinité, et peu de temps après à Chacachacare et à Monos, deux îlots situés entre l'île de la 'Trinité et le Vénézuéla ; des sauterelles furent signalées la même année, vers la fin de novembre, à Carénage, an nord-ouest de la Trinité, à $\delta$ "miles» environ $(\mathrm{I} 2,875 \mathrm{~km})$ de Portd'Espagne, la capitale de cette île. En IgI5, en mai également (29-30), des sauterelles apparurent dans les îles de Patos, Chacachacare et Monos et le 4 juin suivant, un exemplaire est parvenu jusqu'à Port-d'Esspagne (URICH). Dans l'Argentine, l'époque d'apparition des adultes subit des variations appréciables d'une année à l'autre, selon les divers factenrs qui interviennent pour la hâter ou la retarder; les sauterelles adultes apparaissent entre les mois de juillet et de septembre et même après dans les provinces du nord, les premières envahies. Dans les cas d'invasions précoces, la "langosta" couleur brique a un vol élevé, elle parcourt de longues distances et elle est vorace; lorsque, par contre, les invasions sont tardives, 1'insecte est de couleur jaunâtre, et le vol en est bas et court; il mange pen et ne tarde pas à pourvoir à sa reproduction.

Entre la date d'apparition des sauterelles dans les régions du nord et dans celles du sud (province de Buenos-Aires et territoire de La Pampa) il s'écoule d'ordinaire une période de temps que l'on peut évaluer à trois ou quatre mois, durant laquelle les bandes envahissantes font leurs incursions et leurs pontes dans les zones intermédiaires. Au Paraguay, l'apparition des ailés a lieu le plus généralement en septembre et moins fréquemment en août et en octobre, dans la zone occidentale ou Chaco; l'invasion de la partie orientale se produit surtout, d'ordinaire, en octobre. Il est rare qu'il y ait une deuxième invasion à une autre époque de l'année. 
Dans l'Uruguay, l'époque de l'apparition de la "langosta" varie selon la température de 1'hiver ; en Igr 4, par exemple, année oì 1'hiver fut peu rigoureux, les premières invasions eurent lieu de très bonne heure, à savoir dans les derniers jours de juillet. Mais, en général, c'est au mois d'aô̂t que sont signalées les premières apparitions d'adultes.

D'après AlfFARO, 1'accouplement a lieu peu après l'apparition des adultes, et dure, d'ordinaire, quelques heures ; pendant cette période les femelles prennent une teinte châtaine, tandis que les mâles, plus petits, sont toujours d'une couleur plus claire. Toutefois on a observé à Costa-Rica, en Igr5, que dans des lieux élevés, comme le Zarcero, où les sauterelles furent retenues plusieurs jours, à cause de l'altitude au-dessus du niveau de la mer, des pluies fréquentes, des brouillards et de la basse température, elles ne se livrèrent jamais à la reproduction; les mêmes observations furent faites sur des exemplaires recueillis dans le Naranjo de Alajuela et tenus en captivité pendant plus de trente jours. Il n'en fut pas ainsi dans les localités situées à moins de $500 \mathrm{ml}$ d'altitude (Balsa, Escobal, Orotina, province de Puntarénas, Guanacaste) où l'insecte séjourna plusieurs semaines précisément pour procéder à l'accouplement. Durant cette période, les sauterelles se posent sur des terrains nus ou n'ayant qu'une rare végétation, dans les sillons des terrains ensemencés ou sur les plantes peu élevées, toujours réunies en grand nombre; il semble alors - écrit ALFARo - qu'elles ne s'occupent guère de se nourrir, et elles se laissent prendre avec les mains, car elles ne s'envolent qu'à de courtes distances. D'après d'autres, au contraire, à leur arrivée dans une nouvelle localité, les sauterelles se nourriraient avec une grande voracité, puis s'accoupleraient. Dès qu'elle est fécondée, la femelle creuse avec son abdomen un trou, le plus souvent dans les endroits où le terrain est meuble et friable, et y pond ses œufs. Un exemplaire capturé à Orotina vers la moitié de juillet en pondit soixante; d'abord jaunes les œufs deviennent ensuite bruns. D'après ce que rapporte F. W. URICH, il est probable que les adultes vivent deux ou trois mois, durant lesquels ils pondraient leurs œuts. Dans l'A rgentine, l'accouplement de cette sauterelle est précédé, paraît-il, d'un état de lourdeur de l'insecte; cet état est caractérisé par le vol court et bas des bandes, et l'acte s'accomplit dans les endroits mêmes qui serviront à la ponte, à savoir les terrains de consistance moyenne, comme les bords des routes et les lieux où la végétation est plutôt rare. Pendant leurs invasions annuelles dans l' Argentine, ces sauterelles pondent normalement de trois à cinq fois dans les direrses régions que leurs bandes parcourent en se dirigeant vers le sud-est ou le sud. Le nombre des œufs, qui est approximativement de cent pour la première ponte, diminue jusqu'à soixante dans les pontes suivantes. A moins qu'un ouragan ou une autre perturbation atmosphérique ne les oblige à agir autrement, ces sauterelles choisissent aussi dans 1'U r 11 g u a yّ les endroits dépourvus de plantes, entre autres les bords des routes peu fréquentées, pour y pondre leurs œufs. La ponte peut avoir lieu du mois d'août au mois de janvier suivant.

A Costa-Rica la naissance des larves ("saltoncillos ") a lieu après que les cufs ont séjourné dans le sol pendant une vingtaine de jours ; d'a- 
bord blanches, ces larves deviennent brunes en quelques heures et, plus tard, elles prennent une teinte chocolat avec des taches et des rayures rongeâtres et jatınes. D'après URich, les oenfs écloraient en général dans 1'espace de trois mois on davantage, selon les conditions d'humidité prévalant à ce moment-1à. Dans l'Argentine, la durée de l'incubation varie d'après les régions et les époques où la ponte se fait; la chaleur et une humidité relative du sol l'accélèrent, tandis qu' nne température fraîche et une sécheresse prolongée la retardent. Des pluies excessives peuvent en arrêter totalement l'évolution vltérienre. L'éclosion se produit entre le vingtième et le cinquantième jour après la ponte dans des conditions climatiques normales et suivant 1a saison, car il faut de 45 à 50 jours en hiver, de 30 à 40 jours entre le 20 septembre et le I5 novembre, et de 20 à 25 jours à partir de la deuxième quinzaine de novembre. I,es brusques changements de température, les fortes pluies pendant la ponte et cette fonction physiologique elle-même influent beaucoup sur les sauterelles. Dans les endroits où se fait la ponte on rencontre généralement une grande quantité de cadavres ; les bandes diminuent donc notablement chaque fois davantage, de sorte que l'on ne rencontre plus qu'un petit nombre de vieilles sauterelles de la bande-mère mélangées à leur progéniture, lorsque la partie de cette dernière, qui a échappé à la destruction, ayant atteint l'état adulte, reprend son exode vers le nord ou le nord-ouest. En Uruguay, la durée de la période d'incubation des oufs est variable, car la température a sur elle une influence directe. On déduit d'observations qui ont duré plusieurs années, que pour les oufs pondus en août, l'incubation dure cinquante jours; pour ceux pondus du $I^{e r}$ au 20 septembre, quarante-cinq jours ; pour ceux pondus du 20 septembre au Io octobre, quarante jours; pour ceux pondus du ro au 30 de ce dernier mois, trente-cinq jours; pour ceux pondus du 30 octobre au I5 novembre, trente jours ; pour ceux pondus du I5 au 30 novembre, vingt-cinq jours et pour ceux pondus de décembre à janvier, vingt jours. Le froid, pour si rigoureux qu'il soit, n'exerce d'autre action sur les œufs que celle d'en retarder l'éclosion. A leur naissance les larves se nourrissent du peu de matière que les œufs contenaient et même de leur coque.

D'après des observations faites à Costa-Rica, après huit semaines pendant lesquelles 1e jeune Schistocerca subit quatre mues, 1'insecte devient ailé. Sa vie complète dure de dix à douze mois. D'après URICH, les jeunes insectes mettraient à peu près trois mois pour compléter leur évolution et arriver à l'état ailé. En Argentine, dans un intervalle de cinquante jours environ entre la naissance des larves et la dernière transformation, 1'insecte stibit cinq mues pour arriver à 1'état parfait. A leur naissance, les individus sont de couleur claire qui tourne bientôt au gris, puis ( $\mathrm{I}^{\mathrm{er}}$ stade) au gris jaunâtre avec des taches brunes; puis des bandes noirâtres et des taches brun rougeâtres ressortent sur un fond jaunâtre ( $2^{\text {ème }}$ stade) ; plus tard ( $3^{\text {ème }}$ stade), la coloration est encore la même que la précédente, sauf que les taches deviennent foncées; au $4^{\text {ème }}$ stade les santerelles conservent la teinte précédente, enfin an $5^{\text {ème }}$ elles sont de conleur brique avec des rayures et des points clairs et foncés. 
Pendant les dix premiers jours qui suivent leur naissance, les S. partn nnsis (appelés à cette période "mosquita ", petite mouche) ne mangent et ne se meuvent que fort peu et leur caractéristique principale est de se grouper en petites agglomérations faciles à détruire; après cette période, les insectes s' éparpillent çà et là à la recherche de nourriture et, à partir du vingtième jour, ils commencent à former des bandes plus ou moins compactes et en mouvement continuel; à ce stade la "langosta " est remarquablement vorace. D'après les renseignements que l'on possède pour l'Uru g 11 a y, S. paranensis, trois jours environ après sa naissance, passe au stade où il prend dans le pays le nom de "mosquita ". Celle-ci est d'abord jaune pâl€, puis grise et enfin brune. Les caractéristiques de 1'insecte à ce stade sont le peu de voracité dont il fait preuve, la rareté de ses mouvements et sa tendance à se réunir en grandes masses appelées "manchones". Huit jours après sa naissance, la "mosquita " subit sa première mue et prend une teinte gris cendré ; la seconde mue a lieu vingt jours après et l'insecte est alors caractérisé par l'apparition de taches jaunes et noires et par son fort appétit. A l'approche de sa troisième mue, il est devenu plus grand et la formation des ailes commence à se manifester ; cette troisième mue se produit entre le quarante-cinquième et le cinquantième jour à partir de celui où la seconde mue a eu lieu. La voracité de l'insecte à cette période devient énorme. Quelques jours plus tard il est tout à fait prêt à voler.

Schistocerca amcricana. - D'après les observations faites au Y ucatan (Mexique) et applicables, sauf quelques légères modifications, au reste du territoire de la République, l'accouplement a lieu ordinairement le second jour qui suit celui où l'insecte est devenu ailé ; il dure de une à deux heures.

Douze jours après qu'elle a été fécondée, la femelle choisit un terrain dénudé et compact, y creuse avec son abdomen et avec l'aide du mâle qu' elle porte sur son dos, un trou vertical et cylindrique, d'une profondeur de 35 à $40 \mathrm{~mm}$ et $\mathrm{y}$ pond ses oufs en plusieurs couches qu' elle recourre d'un liquide blanc et écumenx; lorsqu'elle a achevé de pondre, elle reconrre encore la dernière couche avec le liquide qu' elle sécrète et, à 1'aide de l'extrémité de son abdomen, elle arrange et aplanit le sol comme pour dissimuler les traces de la ponte. I, e liquide écumeux en se desséchant devient brun et, mélangé avec la terre, il forme, autour des œufs, une enveloppe résistante. Chaque dépôt contient de 60 à Ioo oeufs ; la durée de la ponte varie de une à trois heures.

Quatorze jours après apparaissent les larves, qui mettent de vingt à cinquante minutes pour sortir. Après sept jours a lieu la prenière mue, qui est suivie, de sept en sept jours, d'une seconde, d'une troisième, d'une quatrième et d'une cinquième ; quarante jours envircn après son éclosion, l'insecte devient adulte; il emploie de trente minutes à une heure pour abandonner sa dernière dépotille et être à même de voler; nais il ne s'exerce an vol qu'à partir du jour suivant. 
Schistocerca peruviana. - C'est la seule espèce qui apparaisse au P é ro $u$ avec toutes les caractéristiques de l'insecte nuisible; elle s'y présente à l'état adulte, aux mois d'août et de septembre, si le printemps se présente comme cela a lie11 généralement dans ces vallées profondes, bien abritées et naturellement chaudes; en octobre et en novembre ont lieu 1'accouplement et la ponte: les œufs restent tels quels pendant le mois de décembre suivant; durant tout l'été on rencontre des larves à tous les stades et c'est à cette époque que 1'on constate les plus grands dommages; dès les premiers froids de l'hiver, l'insecte, qui a déjà atteint l'état adulte, commence sa migration et disparaît complètement en juin et juillet pour se diriger vers les points où il se multipliera au commencement du printemps suivant.

Melanoplus spretus. - Les adultes apparaissent an Canada (Manitoba) vers le $I^{\text {er }}$ juillet; dans le nord des Etats-Unis, on les a observés aussi en juin ; ils se montrent déjà en avril dans la partie méridionale ('Texas).

Les femelles pondent de préférence leurs œufs dans les terrains légers et fermes, d'ordinaire dans les champs cultivés ( Ma n i t o b a) et aussi dans les prairies et dans les pâturages, dans les endroits oì l'herbe est plutôt rare ou courte; elles évitent les terrains humides ou mous.

La ponte commence ordinairement en août - très souvent vers la moitié du mois - et continue jusqu'à l'arrivée des gelées, bien que la majeure partie des adultes meure dès le commencement de septembre.

Les œufs sont déposés en petits groupes d'environ 30 chacun, renfermés dans des coques placées à $21 / 2 \mathrm{~cm}$ au-dessous de la surface du sol. La femelle emploie de deux à cinq hetres à la ponte ; dans l'espace de six à huit semaines, elle pond en moyenne trois de ces petits tas d'œufs. Les œufs passent 1'hiver et éclosent l'année suivante.

Au Ma nitoba, les larves apparaissent en avril ou mai; au Texas vers la moitié ou vers le fin de mars.

Après avoir subi plusieurs mues elles deviennent ailées (au Ma n i toba vers juin ou juillet).

En général il n'y a qu' une seule génération par an ; il est rare que les œufs pondus quelquefois en vue d'une seconde génération arrivent à éclore.

Melanop!us bivittatus. - Aux Etrats-Unis et, particulièrement, dans les Etats méridionaux, les adultes apparaissent vers

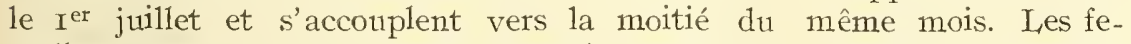
melles commencent à pondre vers le $I^{\text {et }}$ août. Le stade d'évolution de l'insecte, qui précède celui d'adulte, comprend environ dix jours et commence entre le 25 et le 27 juin. La période évolutive tout entière, sauf le temps exigé pour l'incubation, est d'à peu près cent-vingt jours. Dans les régions septentrionales des Etats-Unis ces périodes sont légèrement prolongées et, par conséquent, 1'apparition des adultes est un peu retardée. Toutefois, la différence ne dépasse pas probablement deux semaines. 
Calliptamus italicus. - En France, cet insecte se montre à 1'état adulte en juillet et en août; en Autriche, de juin à septembre.

Les accouplements commencent, en France, à la fin de juillet et ils continuent en août et même en septembre.

En Autriche, sur le Carso gorizien, la ponte a déjà lieu dès la seconde moitié de juin et dure jusqu'à la fin d'août; dans la Da1 matie, elle se fait en septembre.

La femelle pond, à plusieurs reprises, de Ioo à 200 œufs dans les terrains arides, calcaires, légers, incultes et quelquefois aussi, s'ils ne sont pas trop humides, dans les terrains cultivés (Fr a n ce); dans les prairies permanentes, dans la terre ou dans les tas de pierres (Autriche).

I a femelle, après avoir creusé, à l'aide de son abdomen, un trou dans le sol, à une profondeur de 3 à $31 / 2 \mathrm{~cm}$, en revêt les parois d'une substance visqueuse et pond ses œufs dans la mince et délicate cellule ainsi formée (coque ovigère) ; celle-ci n'est pas fermée par un opercule mais bien par une espèce de bouchon formé lui aussi de la matière visqueuse que la femelle sécrète. I,e nombre d' œufs que chaque coque contient est variable : il est, en moyenne, de 30 à 45 en France et de 25 en A utriche.

L'hivernage se fait à l'état d' œuf.

L,es larves éclosent vers la fin de mai ou au commencement de juin (France), et d'avril à mai (Autriche).

Après une période de 35 à $4 \mathrm{I}$ jours en France et de 6 à 8 seniaines en Autriche, l'insecte, qui a subi cinq mues, devient ailé.

L'évolution de cette même espèce en Italie et en Grèce ne diffère pas beaucoup de celle qui a déjà été indiquée pour Dociostaurus maroccanus dans ces pays. Il faut noter seulement, en ce qui concerne l'I t a 1 i e, que $C$. italicus y apparaît après $D$. maroccanus (c'est-à-dire en mai) et qu'il y est moins nombreux que ce dernier. Les deux espèces deviennent adultes entre le soixantième et le soixante-dixième jour.

\section{MGEUR GÉNÉRALES DES SAUTERELLES}

En général, qu'ils appartiennent à la catégorie que l'on peut appeler des grands migrateurs ou à celle des petits migrateurs dont on parlera encore plus loin, ces insectes, à peine nés, et pendant les premiers jours de leur vie larvaire, vivent groupés en bandes plus ou moins nombreuses. I,e plus souvent ils petuvent déjà sauter quelques heures après l'éclosion; toutefois, pendant les premiers jours, surtout si la nourriture ne leur manque pas, ils ne s'éloignent pas des lieux où ils sont nés, ou bien, s'ils parcourent de courtes distances, ils se menvent tous, d'ordinaire, dans la même direction. Il n'est pas rare de trouver dans ces bandes des larves à divers degrés d'évolution, ce qui dépend du fait qu'elles ne sont pas écloses toutes en même temps ou bien que des bandes d'âge différent se sont mêlées; il arrive aussi quelquefois que l'on trouve mélangées des larves appartenant à des espèces différentes d'une même famille ou même à des familles diverses; par exemple, des 
représentants de Phasgonuride mêlés à des représentants de Locustidce. A cette période, les jeunes insectes se montrent déjà très voraces.

Quelques jours après, les jeunes orthoptères se meuvent avec plus d'agilité, surtout le matin, lorsque le soleil est déjà haut et que, par conséquent, la température est douce; et, après avoir fait de plus longs parcours, ils s'éparpillent pour se nourrir lorsqu'ils ont trouvé une localité convenable et cela jusqu'à ce que l'air commence à fraîchir. Ils se réunissent alors de nouveau et lorsque le soleil est sur le point de se coucher, ils grimpent sur les herbes, sur les buissons, sur les pierres, sur les murs, et ils s'y rassemblent en grand nombre pour se mettre à 1'abri de l'humidité de la nuit. Parfois cependant, surtout si la température est chaude, les jeunes insectes passent la nuit sur le sol ou sur les herbes basses.

Ils restent dans leurs retraites jusqu'au matin suivant, et lorsque le soleil a de nouveau réchauffé l'air, ils reprennent leur route, s'éloignant de plus en plus de leur premier point de départ et allant à la recherche d'une nouvelle pâture; lorsque le ciel est nuageux, ils se meuvent fort peu; s'ils sont surpris par une pluie violente ou par un vent impétueux, ils cherchent un abri sous les plantes environnantes, sinon ils s'éparpillent et attendent le soir pour se grouper de nouveau dans de nouvelles retraites et y passer la nuit.

A mesure que les jeunes insectes grandissent, leur voracité augmente, de sorte qu'ils détruisent toutes les plantes qu'ils rencontrent sur leur passage et qu'ils sont sans cesse à la recherche d'aliments nouveaux et plus abondants; c'est ainsi qu'ils abandonnent le plus souvent les prairies et les pâturages pour envahir les semis. C'est précisément la grande voracité qui se manifeste durant le cours de leur évolution qui rend les jeunes plus redoutables que les adultes. Ils s'avancent souvent sur un large front, mais ils marchent ordinairement en bandes longues et étroites.

En général, les obstacles sont surmontés avec habileté et avec une célérité relative, car les jeunes insectes en marche ne changent pas facilement la direction prise par la bande.

On cite des cas où ils n'ont même pas reculé devant des étendues d'eau rencontrées sur leur passage. Au mois de juin Igor, par exemple, on observa dans la province de Cacétès (Espagne) qu'une bande consisidérable - elle avait $500 \mathrm{~m}$ de largeur sur environ $5 \mathrm{~km}$ de longueur arriva près d'une lagune de $800 \mathrm{~m}$ de large située dans le territoire municipal d'Arroyo del Puerco et chercha à la traverser sans toutefois y réussir. L'agglomération des insectes était telle que, s'étant avancés dans l'eau où ils se noyèrent, la lagune, le second jour de 1'apparition de la bande, était littéralement couverte de cadavres sur un grande partie de sa surface, mais on pouvait encore se rendre compte de la direction invariable que la bande avait prise. Nous ajouterons que deux ou trois jours après, il s'exhalait de cette énorme masse en putréfaction des odeurs pestilentielles tellement fortes qu'on pouvait les sentir à un kilomètre de distance.

On a constaté plusieurs fois que des bandes sont passées d'une rive à l'autre du 'Tirso, le plus grand fleuve de la Sardaigne. Dans ces cas, les pre- 
miers insectes qui passent périssent souvent et servent de pont à ceux qui les suivent.

Dans l'Uruguay, on cite également le cas d'une bande composée d'insectes qui n'élaient pas encore munis de leurs ailes, qui a réussi à traverser le fleuve Uruguay sur une largeur approximative de deux kilomètres.

Il y a beaucoup d'autres exemples de l'habileté de ces insectes à la nage. On a observé, dans les Etats Fédérés Malais, que de jeunes orthoptères arrivés à peine au second stade de leur évolution, après avoir traversé un cours d'eau rapide de I4 m de large environ, et être arrivés dans les champs de riz voisins, sautèrent de nouveaut dans l'eau et, nageant de plante en plante, purent ainsi en détruire les feuilles et les tiges jusqu'au niveau de l'ean et parfois même au-dessous de ce niveau.

A mesure que ces orthoptéres se rapprochent de l'époque de la dernière mue, après laquelle ils deviennent insectes parfaits ou ailés, ils paraissent plus actifs dans leurs mouvements, plus rapides dans leurs déplacements d'un point à un autre et aussi beaucoup plus roraces; mais, le moment de la métamorphose étant arrivé, ils cessent de manger, grimpent sur les plantes ou sur d'autres supports un peu élevés, ou bien ils se cachent entre les hẹbes et dans les anfractuosités du sol et ils y restent immobiles, la tête en bas. jusqu' à ce qu'ayant abandonné la dépouille dont ils étaient revêtus, ils apparaissent adultes et munis de leurs ailes encore molles et mal étendues. D’abord engourdis, ils reprennent déjà le deuxième jour leur mobilité et leur vivacité et acquièrent aussi en même temps uné voracité considérable, qui diminute beaticoup de nouveaus à l'époque de l'accouplement et de la ponte, au point de laisser quelquefois intactes des cultures qu'à un autre moment, ils auraient vivement convoitées.

Le second ou le troisième jour après qu'ils sont devenus adultes, ces insectes ont déjà leurs ailes complètement étendues ; lorsque l'air est réchauffé par les rayons du soleil, les sauterinles commencent à voleter çà et là pour sécher leurs ailes rendues humides pendant leur séjour nocturne sur les arbres, les arbustes, etc. Elles ne tardent pas à descendre, à chercher leur nourriture, puis elles reprennent leur vol, en effectuant des tours circulaires pour revenir aux endroits d'où elles étaient parties et y passer la nuit rétnies en groupes dans les plantations, à proximité des bois, dans les enclos, partout où il y a de la végétation sur laquelle elles peuvent se poser. souvent la tête tournée vers le haut. Filles se groupent parfois en si grande quantité sur les plantes, que celles-ci en sont littéralement convertes et le nombre d'individus qui s'y sont posés est tellement élevé que les plantes se courbent sourent sous leur poids; parfois même des plantes oul des branches assez grosses et robustes se brisent.

Le jour suivant, les sauterelles s'envolent de nouveau à des hauteurs quelquefois très considérables et, rémnies en bandes plus ou moins noulbreuses, elles peuvent dès lors se transporter avec nne grande facilité d'une localité à nue antre plus ou moins éloignée en semant alors la destruction autour d'elles; et, dès qu'elles ont trouvé dans les territoires enrahis un endroit propice, elles s'apprêtent le plus souvent à l'accouplement et à la ponte. Après quoi, dit-on généralement, elles ne tardent pas à mourir; par contre, 
d'après certains auteurs, elles s'envoleraient de nouveau.: surtout pendant la nuit, pour passer dans d'autres régions.

La distance que les vols peuvent parcourir est des plus variables et parfois réellement étonnante. L,es grands migrateurs - par exemple, Schistocerca tatarica, S. americana, etc. - arrivent à dépasser plusieurs milliers de kilomètres. Les petits migrateurs - Dociostaurus maroccanus, Melanoplus spretus, Calliptamus italicus, etc. - ne franchissent pas, en général, des distances supérieures à $I 0,20$ et $30 \mathrm{~km}$. I a distance moyenne que peut parcourir en un jour $M$. spretus est de $32 \mathrm{~km}$ environ; mais, dans des conditions favorables, il peut dépasser de beaucoup cette distance: en effet, lorsqu'au commencement de l'époque du vol, les individus de cette espèce traversent la région des steppes des Etats-Unis, et qu' un fort vent les favorise, ils peuvent parcourir en un seul jour de 320 à $480 \mathrm{kn1}$.

Quoique la direction d'une bande, composée d'individus non ailés on ailés, soit, le plus souvent, comme nous l'avons déjà dit, difficile à changer une fois qu' elle s'est mise en marche, cependant ces insectes ne se dirigent pas toujours et tous vers un point cardinal déterminé et ils ne suivent pas toujours non plus, comme on le croit généralement, la direction du vent.

On a observé également, que, dans leurs déplacements, certaines sauterelles peuvent voyager en volant même la nuit, et qu'elles peuvent aussi se nourrir, pendant la nuit, bien que le plus souvent ces insectes restent dans leurs retraites durant les heures nocturnes ; on a noté encore que les sauterelles suivent souvent dans leur vol les bassins des fleuves ou les cours d'eau; on en a vu parfois se poser sur la surface de l'eant et continuer ensuite leur vol après avoir acquis une nouvelle vigueur.

MODALITÉS DES APPARITIONS ET DES MIGRATIONS DES DIVERSES ESPİCES DANS LES DIFFÉRENTS PAYS

Nous allons examiner, en prenant pour guide les documents que nous avons eus à notre disposition, quelles sont les modalités d'après lesquelles ont lieu, dans les divers pays, les apparitions, les déplacements et les migrations des différentes espèces de sauterelles.

Dociostaurus maroccanus. - Ein Portuga1, les individus de cette espèce arrivent par grands vols de 1'Esspagne, venant de la rive gauche du Guadiana.

Quant à 1'E spagne, il faut considérer D. maroccanus comme localisé dans ce pays : la région qu'il habite en permanence comprend les provinces de la partie centrale et méridionale du payś, à savoir les provinces d'Abacète, Avila, Ciudad Real, Cuença, Madrid, Salamanque, Tolède, A1méria, Badajóz, Cacérès, Cadix, Cordoue, Huelva, Jaen, Malaga et Séville.

En I talie, où l'apparition de nombreux et puissants vols de sauterelles, constitués principalement de $D$. maroccanus, de Calliptamus italicus et de Tettigonia albifrons, est intermittente et sporadique, on ne se trouve pas, généralement, en présence de véritables invasions suivant des directions déterminées comme cela se produit dans d'autres pays du monde. I.es 
historiens font mention d'invasions de sauterelles en Italie; mais, même en admettant que leurs assertions soient exactes, on doit estimer qu'il s'agit d'invasions extraordinaires et tout à fait occasionnelles de Schistocerca tatarica venant d'Afrique. Mais de semblables invasions ne se sont jamais produites dans ces cent dernières années. Les sauterelles, en ce qui concerne ce pays, doivent donc être considérées comme autochtones. Flles y naissent et elles s'y multiplient à la dérobée, jusqu'à ce qu'elles passent d'une localité à une autre très voisine. Mais parfois, et le plus fréquemment aux mois de juillet et d'août - en Sicile généralement ce dernier mois et quelquefois même en septembre - les sauterelles, si le vent les aide, franchissent des distances de 20 à $30 \mathrm{~km}$ et c'est seulement alors qu'il peut être question d'invasions. D'ailleurs, ces invasions ne se répètent pas fréquemment, et ne suivent pas des modalités constantes.

En Ho n g r i e, il n'y a pas lieu non plus de parler d'invasions en ce qui concerne D. maroccanus. Celui-ci appartient probablement à la faune originaire du pays. Il est probable qu'il s'y trouvait bien avant la régularisation du cours des fleuves principaux et le dessèchement des régions inter-fluviales. Mais, comme à ce moment les circonstances n'étaient pas favorables à sa multiplication, sa nocivité ne se présentait pas d'une manière évidente. Dès que le milieu a été changé, l'insecte a tronvé des conditions propices pour se développer et il est devenu un vrai fléau pour le pays oì il se renouvellera très probablement d'une manière périodique. En somme i1 devient nuisible là où il se développe ou dans les alentours immédiats : il change quelquefois de région; mais ces changements ne sont pas importants et ils ne sont pas comparables à cenx que l'on constate dans d'autres pays.

Bien qu'il soit apparu à diverses reprises en $\mathrm{R}$ o $\mathrm{u} \mathrm{m}$ a $\mathrm{n} \mathrm{i} \mathrm{e}, \mathrm{D}$. maroccams n'y a causé que des dommages relatifs. Cette espèce se trouve à 1'état endémique surtout dans le district de Tulcéa, sur les plateaux, dans le voisinage des forêts, dans les prairies. Il a été signalé également dans le district de Romanati.

En Grèce, les sauterelles - principalement D. maroccanus et Calliptamus italicus - apparaissent en grande quantité tous les denx out trois ans, puis elles disparaissent pour réapparaître quelques années après. Elles se déplacent d'un point à l'autre parfois même à des distances notables ; pendant ces vols, elles suivent en général, d'après ce que 1'on dit, la direction du vent.

D. maroccanus doit être considéré dans 1' Afrique du Nord comme une espèce autochtone, localisée, indigène. Son habitat normal est dans les Hauts-Plateaux de l'Atlas; il vit ordinairement dans les steppes et ce n'est que lorsque sa multiplication devient extrêmement abondante qu'il sort de son centre; on constate alors la présence de cet orthoptère dans les régions situées autour de son habitat normal.

Locusta pardalina. - Le principal territoire de reproduction de cette sauterelle, qui envahit le Sud-Ouest africain allemand. se trouve, d'après ce que l'on affirme, dans le désert de Kalahari. Dans 
1'Union de 1'Afrique du Sud, qui est envahie aussi par le même orthoptère ; on estime que cette espèce se tronve surtout dans le voisinage de la partie méridionale de ce désert, qui paraît un centre d'où partent des bandes migratrices se dirigeant vers 1'onest, le sud et l'est.

Locusta migratoria. - D'après ce que l'on sait des plus anciennes invasions de sauterelles en Roumanie, qui datent de I538, les bandes qui dévastèrent ce pays sont considérées comme venant de la Bulgarie actuelle. Dès lors on savait que ces insectes se trouvaient à l'état endémique dans les régions du delta du Danube, terrains très propices à leur évolution. Du delta, les bandes rayonnaient un peu partout dans le nord et dans le sud de la Roumanie. Ajoutons que des dommages considérables furent causés à ce pays par des invasions provenant du sud de la Russie.

La Roumanie est encore aujourd'hui sous la menace d'incursions de sauterelles venant des pays limitrophes.

Comme nous avons déjà et l'occasion de le rappeler, L. migratoria apparut en vols considérables, en I880, au J a p on (région de l'Hokkaido) et $\mathrm{i} 1 \mathrm{fut}$ alors considéré comme venant du continent. Il y fit une deuxième apparition 1'année suivante. En I905 et en Igo6, on le signala dans les îles Ogasawara (îles Bonin) et il s'y est montré depuis chaque année en quantités plus ou moins notables. Réunis en bandes les individus de cette espèce passent facilement au-dessus des montagnes élevées ou des cours d'eau; ils suivent la direction du vent à une vitesse de 6 "chô" par minute (654 m environ). Leur vol n'est pas toutefois de longue durée; on estime que la distance qu'ils peuvent parcourir varie de 30 à 60 " ri 》) (II $8-235 \mathrm{~km}$ environ) par an. On ne croit pas possible que cet insecte passe du Japon sur le continent et s'y multiplie.

La direction suivie par les L. migratoria, qui envahirent en septembre IgI4 la République de Sa1vador, était celle du nord-ouest; elles venaient du Mexique et dı Gratémala.

Pachytylus sp. - Il n'y a rien de vraiment certain au sujet de l'origine des sauterelles dans les Etats Fédérés Ma1ais. Toutefois, on est porté à croire qu'il s'agit, non pas d'un insecte qui se serait multiplié soudainement in situ d'une manière extraordinaire, mais bien d'un orthoptère importé ; et cela surtout si l'on ne perd pas de vue que sa présence fut signalée, an début, dans un port (Port Dikson), localité qui paraît moins indiquée pour la reproduction d'un insecte indigène que beaucoup d'autres qui se trouvent dans ces Etats, et, de plus, si l'on considère que la multiplication et la dissémination du fléau vers l'intérieur eurent précisément leur origine dans cette localité maritime.

Lorsqu'elles commencèrent à se répandre, les sauterelles suivirent une direction à peu près définie, du sud au nord; elles passèrent de 1'Etat de Négri-Sembilan dans celui de Sélangor; avec le temps, elles passèrent de ces deux Etats dans le Pahang.

Pendant I9I4, et en particulier d'avril jusqu'à octobre, on observa dans 
tout le territoire une tendance à un mouvement général des sauterelles vers le nord-ouest, tandis que pendant le reste de l'année la direction fut intervertie.

Le même fait fut constaté de nouveau au commencement de IgI5: par exemple, les quelques bandes qui se trouvaient dans le Sélangor et qui y étaient venues du Négri-Sembilan, revinrent plus tard, au moins en partie, dans ce dernier Etat.

A Malacca et dans 1'Eitat de Johore, les sauterelles sont venues des Etats Fédérés Malais. D'après ce que 1'on a observé à la fin de février I0I5, presque tontes les bandes qui s'étaient localisées dans la partie septentrionale du Johore s'en éloignèrent en se dirigeant vers le nord. pour revenir, en partie, à leur point de départ, au mois de mars, et y pondre leurs cufs.

Oxy:a velox. - C'est un orthoptère qui, au J a pon, se limite en général, à la localité où il se manifeste et il ne s'en éloigne pas tant qu'il y tronve une nourriture suffisante. Comme cette condition ne fait jamais défaut, les migrations de cet insecte sont absolument inconnues au Japon.

Cyrtacanthacris sucuincta. - Cette sauterelle se reproduit très abondamment dans 1'In de, dans le voisinage des Ghats occidentaux, le long d'une ligne formée par des collines boisées qui côtoient la mer du district de Thana jusqu' à Coorg ; l'habitat principal de reproduction est aux environs de Goa, pas dans la forêt. mais dans les prairies ouvertes qui l'avoisinent. Cet insecte se reproduit aussi sur toutes les plaines de l'Inde méridionale, dans les localités qui lui conviennent, et on le trouve dans 1'Assam, à Bourma et dans toute l'Inde péninsulaire. Lorsque le nombre des individus atteint une certaine importance, les insectes deviennent migrateurs; au demeurant, C. succincta est une santerelle ordinaire localisée qui fait partie de la faune régulière de la plaine.

Des Ghats occidentaux les bandes se répandent à Bombay, à My̆sore. dans certaines parties de Madras, à Hyderabad, dans les Provinces Centráles et dans quelques parties de 1'Inde centrale.

Cyrtacanthacris septemfasciata. ... La Rhodésia méridionale est considérée comme pouvant être parfois envahie par cette sauterelle, qui provient du nord du Zambèze.

D'après les renseignements que 1'on possède au sujet de la Province de Moza m bi qu e, C. septemfasciata - 1'unique espèce qui intéresse vraiment ce territoire - est répandı le long de la côte orientale africaine, du Natal jusqu'au nord du fleuve Rovouma ; dans les années où il abonde, il gagne les terres basses du 'Transvaal et de la Rhodésia méridionale et il s'étend quel quefois dans le sud jusqu'à la côte du Cap; il n'atteint que rarentent les terres hautes de 1'Afrique du Sud. Bien que cette santerelle ne présente pas les caractéristiques d'insecte migratenr si accentuées chez Locusta par dalina, il est certain qu'elle préfère quelques localités qui constituent jusqu'à un certain point ses quartiers d'hiver; d'autre part, il n'est pas rare de ren- 
contrer, pendant les cycles de plus grande intensité de cet orthoptère, des bandes sporadiques hivernant çà et là partout où les circonstances leur sont favorables.

C'est ainsi que cette sauterelle parait être endémique dans le Zoulouland et dans le territcire de Barue, y compris une partie de la région située au nord dı Zambèze et de la Rhodésia méridionale. Du Zoulouland, elle émigre dans le Natal et daus les districts de Lourenço-Marques et d'Inhambane; elle occupe parfois le territoire du Souaziland, la partie basse du 'Transvaal et la Rhodésia méridionale. Du foyer du Zambèze, elle vole vers la côte en suivant le cours du fleuve et occupe une partie du district de Téte, une partie du Nyassaland. et presque tout le district de Quélimane.

Ses foyers préférés sont constitués par des vallées humides et chaudes c'est pourquoi, étant données les conditions topographiques et climatoIogiques générales de la région du littoral en question, il n'y a pas lieu de s'étonner que l'instinct migratoire soit peu accentué chez cette espèce, car rien ne paraît lui imposer un retour forcé vers ses quartiers d'hiver primitifs.

D'après les observations faites dans l'Union de l'Afrique du Sud, l'babitat permanent de cet insecte dans ses rapports avec 1'Afrique du Sud serait encore inconnu; 1'idée que cette espèce y ait un territoire permanent de reproduction paraît être purement spéculative.

Schistocerca tatarica. - - Cet orthoptère se reproduit dans l'I nde d'une manière permanente dans les collines sableuses et désertiques du Radjpontana occidental, dans le Béloutchistan, dans le sud de l'Afghanistan et de la Perse et il essaime de ces régions en volant sur le Radjpoutana et sur d'autres parties de l'Inde.

Il se peut que le défaut de nourriture développe l'instinct migratoire chez cette sauterelle; des bandes ne tardent pas à se former pour aller à la recherche d'aliments à l'entour, en passant sur le sud-ouest du Pendjab, sur les Provinces Unies, le Bengale, les Provinces Centrales, ils vont à 1'est jusqu'à Assam, dans le sud jusqu'à Madras et au Kathiavar dans la Présidence de Bombay. Il n'est pas rare de trouver dans certaines années le S. tatarica mélangé au Cyrtacanthacris succincta dans le Kathiavar et dans certaines parties de la Présidence de Madras. Les bandẹs qui pénètrent dans les régions plus humides des Provinces Unies, des Provinces Centrales, de Bombay, Bengale, Madras et Assam paraissent mourir en grande partie avant d'avoir pondu. Quant aux vols qui séjournent dans les régions plus sèches du Pendjab et du Radjpoutana, on suppose qu'ils pondent vers la dernière partie de la période des pluies, en aô̂t, septembre et octobre; de même que 1'on suppose que les jeunes insectes atteignent le stade ailé au commencement de la saison froide et constituent les bandes qui pondent leurs œufs l'année suivante, tandis que leurs parents meurent graduellement comme l'ont déjà fait leurs ancêtres au printemps précédent.

On pent affirmer, en ce qui concerne en général $S$. tatarica dans l'Afrique du Nord, qu'il dirige ses invasions du sud au nord, de l'intérieur vers la mer. 
Quant au point de départ de ces invasions sur le continent africain, les auteurs ne sont pas d'accord à ce sujet. I'opinion la plus commune est que ce point de départ initial — c'est-à-dire la "région permanente " des sauterelles - se trouve dans le Soudan central et précisément aux environs du lac Tchad. Selon d'autres auteurs, les bandes proviendraient des régions équatoriales; selon d'autres encore, S. tatarica serait une espèce déserticole qui, dans certaines années, trouvant, vers le nord, des conditions favorables à sa propagation, émigrerait dans cette direction. C'est précisément le Sahara occidental qui, d'après KÜNCKEL, D'HERCUIAIS, constitue la région d'où partent les sauterelles pour se diriger d'abord vers le nord-onest et ensuite vers le nord.

Indépendamment de l'existence de la région permanente du criquet pèlerin dans une partie plutôt que dans l'autre de l'Afrique, il est permis d'admettre que la cause première de ses migrations doit être recherchée dans la nécessité pour lui de trouver ailleurs une quantité suffisante d'aliments, après que, du fait de sa multiplication considérable, qui a lieu précisément dans la région permanente, la nourriture vient à manquer dans cette dernière. I.es vents chauds du secteur sud jouent en outre un grand rôle dans la direction que les bandes migratrices prennent vers le nord. Quant aux bandes provenant des pontes faites dans le nord, elles prennent généralement vers la moitié de juillet une route en sens inverse de celle que les bandes précédentes ont suivie et elles se dirigent vers le sud.

Il faut ajouter, en particulier pour la 'Tunisie, que les vols des sauterelles y arrivent en suivant ordinairement la direction sud-ouest-nordest (région du Djérid) venant du sud-algérien et des oasis du Soûf; cependant les invasions de r8g9 venaient de l'ouest.

De mémoire d'homme, on n'a jamais eu à déplorer dans aucune localité de la Tripolitaine septentrionale des invasions ayant causé des dommages considérables tant aux terrains ensemencés de la steppe (céréales) qu'aux plantations et aux cultures herbacées des jardins. On constate, il est vrai, de temps en temps, tantôt dans une localité tantôt dans une autre, l'existence d'un nombre considérable de sauterelles, surtout dans la steppe, mais ces insectes n'ont jamais présenté les caractères de bandes migratrices.

Ce phénomène pourrait trouver son explication dans le fait que les bandes de sauterelles migratrices se forment, paraît-i1, dans le Sahara occidental et prennent généralement la direction du nord-ouest et du nord: les pays les plus généralement atteints seraient donc le Maroc et 1'Algérie.

Eitant données sa topographie et ses conditions météorologiques, l'Erythrée se prête à être envahie par les sauterelles (S. tatarica) à toutes les époques de l'année. Du printemps à l'automne, ce sont les régions situées sur le haut-plateau qui sont infestées, tandis que les sauterelles passent 1'hiver. dans la plaine et le long du littoral. Les invasions de la Colonie se font du sud et du sud-est par les voies du Tigrai et l'on peut déduire de renseignements constants que la zone permanente éthiopienne est 1'Avergalè. De l'ouest-nord-onest, il y a des invasions qui traversent Kassala et la montagne de Gulza, sans qu'on puisse établir pour le moment 
où se trouve la région permanente d'où proviennent les insectes. En Errythrée, la région du littoral comprise entre Zula-Sahel et la frontière soudanaise est une zone de reproduction hivernale. Il est bon de noter que les sauterelles nées en Erythrée émigrent aı Soudan on en Fithiopie, tandis que celles qui naissent et vivent dans ces deux régions immigrent, échangeant leur territoire selon leurs besoins, soit pour suivre les variations climatologiques, soit pour rechercher des localités favorables à l'incubation et à la nourriture.

Quant à la Nigéria, dans son ensemble, la direction la plus génerale d'oì proviennent les premières bandes de $S$. tatarica (?) qu'on y a observées paraît être celle de l'est. Il en serait de même pouı les provinces méridionales, d'après ce qu'on peut déduire des renseignements dont on dispose à ce sujet. Cependant quelques bandes sont arrivées du nord et ont disparu ensuite dans la direction du nord-est et de l'est; d'autres sont venues de l'onest. Des indigènes se rappellent que quelques-unes de ces bandes retournèrent dans les localités d'où elles étaient venues. Mais il est très probable que c'est de l'est que provenaient le plus généralement les sauterelles qui visitèrent cette Colonie.

Un certain nombre de données ont été recueillies, surtout en 1905, sur les déplacements du $S$. tatarica dans l'Afrique orientale anglaise, mais commè on a signalé l'existence d'un nombre considérable de bandes séparées, les renseignements que l'on possède à ce sujet sont plutôt de nature à engendrer une certaine confusion. Il n'est donc pas possible de suivre les déplacements de chaque bande avec quelques probabilités de certitude. Que l'on veuille bien considérer, en outre, que la Colonie étant placée entre des régions plus ou moins sujettes à la visite des sauterelles, on peut signaler ces dernières, pour ainsi dire, de n'importe quel quadrant. Quoi qu'il en soit, le fait le plus important qui ait été constaté, c'est que Voi est un des territoires de reproduction préférés. La bande de formation récente, qui apparut à Naivasha le 3 février 1905, indiquerait aussi 1'existence d'une zone de reproduction au nord de cette localité.

Quant à l'Afrique orientale allemande, il est établi que les sauterelles arrivent, dans le sud de la Colonie, de 1'Afrique orientale portugaise et que, dans le nord, elles fondent de la steppe sur les territoires habités. Si l'invasion dure longtemps, la reproduction a lieu sur les territoires occupés.

Schistocerca paranensis. - Costa-Rica doit être plutôt considéré comme un lieu de passage pour les bandes de cette sauterelle; elles n'y apparaissent qu'à des époqizes indéterminées et plutôt éloignées l'une de 1'autre et, à ce qu'il semble, elles ne s'y sont jamais arrêtées pendant longtemps; cela tient certainement aux conditions du climat qui leur sont contraires et à l'exiguïté du territoire qui les oblige à considérer le sol de Costa-Rica comme une station inévitable placée sur leur route entre les deux Amériques (AIFARO).

D’après les données les plus récentes, des bandes passèrent de Honduras aur Nicaragua et arrivèrent dans la province de Granacaste, sur 
le territoire de Costa-Rica, vers la fin de I9If. En I9I5, les bandes migratrices reprirent leur voyage vers le sud et envahirent le territoire de la République, en juin, par ses deux versants jussu1'à la frontière de Panama.

AlfFARO a pu observer, au commencement du mois de juin IgI 5 , les bandes qui entrèrent par le nord de la province d'Alajuéla dans les cantons de Zarcero, Naranjo, San Rámon, Palmarés, Grecia et Aténas. Après avoir dépassé le lac de Granada, les vols, d'après cet auteur, se divisèrent en deux colonnes, dont l'une suivit le versant du Pacifique, le long de la côte, sur des terres basses, an pied des montagnes; l'autre se dirigea vers les plaines humides et montueuses de la côte de l'Atlantique, sur les régions de San Carlos et de Sarapiquí et sur la zone à bananiers de la province de Limón ; elle suivit sa route vers le sud sur le territoire de Talamanca pour entrer dans les Bocas del Toro, dans la République de Panama. Mais une partie de cette colonne, qui venait du nord-est, suivit le bassin du fleuve San Carlos où se trouvent des pâturages cultivés et, poussée certainement par les vents, elle se vit contrainte de traverser la chaîne de montagnes dı nord vers la dépression du Zarcero, à une altitude de $1888 \mathrm{~m}$ au-dessus du niveau de la mer; elle avança avec de si grandes difficultés qu'elle fut obligée de s'arrêter pendant quelques jours, et elle poursuivit ensuite sa route au sud, vers la région sud-ouest du platean central.

Quant aux invasions du $S$. paranensis signalées à la 'T rinité, il résulte que les insectes qu'on y a observés venaient du Vénézuéla ; et on suppose que c'est de la Colombie qu'ils seraient venus au Vénézuéla. Pour arriver à la Trinité, les îles situées entre cette dernière et le Vénézuéla leur serviraient d'étapes. Tant que les sauterelles se développeront au T'énézuéla, la Trinité sera toujours menacée d'être envahie par ces orthoptères.

Les zones du territoire du Brésil sujettes aux ravages du $S$. paranensis sont considérées, jusqu'à présent, comme faisant partie de la région temporaire de l'insecte. Elles sont constituées par les Etats de Matto Grosso, Rio Grande do Sul, Santa Catharina, Paraná, São Paulo, Rio de Janeiro, Esspirito Santo et Minas Geraës.

Il résulte de la communication transmise à l'Institut par le Gouvernement de la $\mathrm{B}$ oli vi e que les sauterelles seraient de passage dans nne partie du département de Santa Cruz et dans ceux de Chuquisaca et de Tarija, oi ces insectes sont un vrai fléau pour l'agriculture.

Des bandes y ont été signalées en novembre et en décembre I9I 4 ; dans de nombreuses localités elles détruisirent toutes les cultures de sorte que l'on dut ensemencer de nouveau. Les larves nées des uufs pondus par les santerelles lors de leur passage firent leur apparition avec les nouvelles plantes; elles causèrent de grands dommages dans presque tons les territoires des provinces d'Arce, Avilés et O'Connor.

Au commencement du mois de mai I9I5, de grands vols de sauterelles apparurent de nouveau; leur passage dura de dix à douze jours.

Ën ce qui concerne l'invasion du S. paranensis, constatée au Chili en I906, il résulte que la "langosta " entra dans le territoire de la République par la région du Bio-Bio et que la bande des envahisseurs se divisa en 
deux parties : 1'une se dirigea vers le nord en suivant la vallée du Bio-Bio et l'autre prit la route de l'ouest par Lonquimai, Malacahuello et Curacautin. De violents orages dispersèrent cette partie de la bande. Cette invasion ne produisit que de très petits dommages, ce qui est considéré comme une nouvelle preuve que la sauterelle en question ne peut pas prospérer dans la partie sud du Chili; cela dépend, croit-on, des conditions climatiques du pays, de la topographie de son territoire traversé par de nombreux fleuves et cours d'eau et de la distribution même de la pluie.

D'après ce qu'on rapporte, les vols de $S$. paranensis qui envahissent la République Argentine viennent de sa voisine la Bolivie, et pénètrent dans le pays par la partie occidentale de la frontière du nord, à la hauteur de Yacuiva.

Les bandes envahissantes font leur apparition à des époques plus ou moins précoces, selon que leur marche est favorisée par une température modérée et par les vents du nord ou du nord-ouest ou bien retardée par les pluies, par les basses températures ou par les vents contraires.

D'ordinaire, l'approche de l'invasion est annoncée par de petites bandes qui paraissent jouer le rôle d'éclaireurs.

Quelques vols atteignent des proportions gigantesques; un wil peu exercé pourrait les confondre avec des nuages chargés d'orage, car, à certains moments, ils arrivent à obscurcir le ciel et les insectes couvrent plusieurs lieues carrées lorsqu'ils se posent.

Il arrive parfois que de petites agglomérations d'ailés, détachées des bandes principales nées, sur le territoire argentin, des insectes qui forment les invasions immédiatement antérieures, restent dans les provinces de Jujuy, Salta, Catamarca et La Rioja, où elles sont retenues par des circonstances climatiques adverses, lorsque les bandes susdites prennent leur vol vers le nord-ouest. Ces petites agglomérations hivernent dans les régions indiquées et y pondent leurs weufs entre les mois de mars et de mai.

Les insectes qui naissent de ces pontes sont combattus méthodiquement comme dans les autres parties de 1'Argentine ; toutefois, l'agriculture n'étant pas très développée dans les susdites provinces, la population y étant peu dense et le territoire accidenté, fait de montagnes et des chaînes montagneuses, on comprendra facilement qu'une partie des larves échappe à la destruction. Arrivés à l'état adulte, ces insectes forment de nouvelles bandes; celles-ci prennent leur vol vers les provinces du centre et forment les avant-gardes des invasions qui viennent du dehors et qui sont bien plus considérables que celles purement occasionnelles d'origine intérieure.

Les bandes qui envahissent la République en venant de la Bolivie, de même que celles qui prennent leur vol dans les provinces du nord-ouest de l'Argentine, suivent une direction parallèle, c'est-à-dire du nord-ouest au sud-est et atteignent successivement les provinces de 'Tucumán, Santiago del Estero et la partie nord des provinces de Santa F'é et de Cordoba, qui ne tardent pas à être complètement envahies. Plus tard, ces mêmes bandes s'étendent jusqu'à la province de Buenos Aires et au nord du Gouvernement de La Pampa; toutefois cela ne se produit pas tous les ans.

Une partie des bandes qui envahissent Santa Fé pénètrent parfois 
jusqu'à Corrientes et Entre Ríos et de petites agglomérations des sauterelles qui attaquent Cordoba passent dans la province de San Luis, d'où elles s'étendent, bien qu'exceptionnellement, jusqu'aux provinces de San Juan et de Mendoza. Il est rare que les invasions s'étendent plus au sud du territoire de La Pampa Centrale.

Outre le courant d'invasion que nous venons de décrire et qui est le courant normal qui se produit fatalement chaque année, s'étendant plus ou moins selon l'ordre et la forme indiqués, il en existe parfois un autre qui, de même que le premier, provient de la Bolivie. Les bandes qui le composent pénètrent dans le Paraguay et de là, passant ensuite à travers les territoires de Formosa et du Chaco, elles fondent successivement sur les provinces de Santiago del Esstero, Corrientes, Cordoba, Santa Fé, Entre Ríos et Buenos Aires. Les invasions que l'on signale dans l'Uruguay et dont l'intensité est variable proviennent de ce courant.

D’après ce qu'on rapporte pour le Paraguay, les sauterelles ( $S$. paranensis), suivant leur route habituelle, se dirigent en majeure partie vers le sud ; mais une partie, la moins nombreuse, prend la direction de l'est et, passant sur les forêts élevées du Haut-Paraná, presque toujours à de grandes hauteurs, les sauterelles se dirigent, saus toucher ces forêts, vers le Brésil où elles se posent sur les premières savanes des Eitats de Paraná et de Santa Catharina et se répandent quelquefois dans ceux de Rio Grande ou de São Paulo.

Les invasions seraient devenues plus fréquentes et plus nuisibles au fur et à mesure que diminuaient les terrains boisés et l'humidité de l'air. 'Toutefois, les dommages n'ont jamais pris, au Paraguay, les proportions qu'ils atteignent dans d'autres parties de 1'Amérique du Sud; cela dépend de ce que, au Paraguay, les sauterelles manquent complètement ou ne se présentent qu'en petit nombre pendant plus que la moitié de l'année, et aussi du fait que sept, dix ans et même davantage s'écoulent sans que les sauterelles y apparaissent, et si elles s'y montrent ce n'est qu'en vols de pet1 d'importance.

D'après le rappo1t transmis par le Gouvernement de 1'Urug t1ay , les bandes de $S$. paranensis qui envahissent périodiquement le territoire de 1a République, proviennent presque toutes de 1'Argentine; cependant, certaines années, des vols arrivent du Brésil. Les sauterelles qui viennent de 1'Argentine pénètrent dans 1'Uruguay en traversant au vol le fletıve Uruguay; on a observé des cas où l'insecte n'ayant pas encore atteint l'état adulte a passé le fleuve à la nage sur une distance approximative de det1x kilomètres. Les bandes suivent la direction du nord-onest, du nord et de l'ouest et, à leur entrée dans la République, elles se dirigent, sanf les conditions météorologiques spéciales du moment, vers le sud et le sud-ouest.

Avant d'aller plus loin, il y a lien d'exposer ce que l'on connaît jusqu'à présent au sujet de la région permanente du S. paranensis.

La situation de cette région a préocctipé, comme on doit le supposer, les autorités de l'Argentine.

On crut d'abord que cette région pouvait se tronver dans les territoires boisés du Chaco et de Formosa; on constitua donc des Commissions 
d'étude qui, en deux occasions différentes et à des époques oì le reste du pays était exempt de sauterelles, parcoururent en divers sens les territoires indiqués; mais ces Commissions, se basant sur leurs observations directes corroborées par les renseignements rectieillis sur place lors de leur passage, arrivèrent à conclure que la région permanente de cette sauterelle ne devait pas être recherchée dans les territoires visités.

L'entomologiste L YNCH ARRIBÁLZAGA, qui avait fait partie des susdites Commissions, fut informé plus tard par une personne qui voyageait fréquemment entre la Bolivie et 1'Argentine, qu'il y avait un point de la province bolivienne des Cordillères où 1'on tronvait la sauterelle toute l'année ; cela confirmait, en quelque sorte, 1'opinion de cet entomologiste, à savoir que la région permanente devait être située au nord de la République Argentine et hors de son territoire; il proposa alors d'organiser une expédition officielle qui se rendrait dans cette région et en établirait, si possible, les limites.

LYNCH ARRIBÁLZAGA partit 1e 29 avril I908 de Jujuy et suivit 1'itinéraire suivant: Jujuy, Yacuiva, San Firancisco, Lagunillas, Abapó, Santa Cruz et retour par Cochabamba.

Se basant sur les renseignements fournis par les autorités des localités qu'il traversait et par quelques particuliers, ainsi que sur les faits qu'il avait lui-même observés, L,YNCH ARRIBÁLZAGA traça les limites de la région qu'il considère comme permanente; il la plaça entre le $19^{\mathrm{e}}$ et le $22^{\mathrm{e}}$ degré de latitude sud et $1 \mathrm{e} 6 \mathrm{I}^{\mathrm{e}}$ et $67^{\mathrm{e}}$ degré de longitude occidentale (provinces boliviennes des Cordillères et du Chaco).

LYNCH ARRIBÁLZAGA ne pénétra pas dans la région qu'il a décrite, il l'aborda setılement du côté occidental; l'expédition fut courte et le nombre des renseignements obtenus est pen abondant, de sorte que pour arriver à des conclusions définitives sur la détermination de la région permanente du $S$. paranensis qu'il a tracée, il est nécessaire d'entreprendre des études complémentaires et de pénétrer plus avant dans les provinces orientales de la Bolivie.

Quoi qu'il en soit, l'expédition de L YNCH ARRIBálZaGa a permis d'affirmer - d'après le rapport envoyé par le Gouvernement de l'Argentine -que c'est de l'est de la Bolivie et d'une région égale à celle qui a été signalée out plus étendue, située là oì elle a été indiquée on dans son voisinage immédiat, que rayonnent les invasions qui fondent sur le reste de la Bolivie par l'ouest et par le nord ; par l'est, elles arrivent dans l'Eitat de São Pan1o dı Brésil et dans la République du Paraguay et par le sud dans la République Argentine; les bandes partent approximativement à la même époque et les jeunes insectes nés des cufs pondus par ces bandes envahissantes reviennent au point de départ à une époque uniforme, ce qui peut faire soupçonner l'existence d'une zone d'irradiation et de concentration dı S. paranensis, en Bolivie.

An Brési1, on est d'avis que la région permanente de cette santerelle se trouve dans la partie orientale de la Bolivie. On a à pen près la même opinion à cet égard en Uruguay, car on y suppose que la région permanente est située au centre de l’Amérique du Sud. 
Par contre, le rapport transmis par le Gouvernement du P a r a u a y, exprime la conviction que si cette région permanente existe, elle doit être beaucoup moins étendue que ne le suppose L YNCH Arribáizaga. Fin effet, une partie de la région que ce dernier considère comme telle est iouverte de bois et, par cela même, peu propice à une multiplication abondante des sauterelles; une autre partie renferme des marais salants ou des terres très salées ; une autre enfin est une steppe ouverte à tous les vents et relativement froide en hiver, époque à laquelle les sauterelles ont besoin de chaleur. On estime au Paraguay qu'il est très probable que le centre d'irradiation du fléau n'existe ni dans le Chaco ni ailleurs, puisque les pontes ont lieu en Argentine et dans n'importe quelle localité.

Schistocerca americana. -- Les plus grandes invasions de cette sauterelle qui ont désolé le Mexi qu e ont eu lieus souvent, sinon toujours, de trente en trente ans ; entre ces invasions, on en a constaté d'autres moins importantes et de caractère local. Chaque fois que la République n'a pas subi d'invasions pendant quelques années, on a eu des invasions partielles dans les pays de l'Amérique centrale et on a observé, dans ces cas-là, que les bandes se multipliaient jusqu'au commencement de la grande invasion périodique. Alors, les bandes se réunissaient et s'avançaient vers le nord jusqu'à la frontière mexicaine ; là, elles se divisaient en deux parties: 1'une se dirigeait vers le nord et l'autre prenait la direction du nord-ouest parallèlement à la côte du Pacifique. Arrivées sur les Etats de Chiapas ou de Tabasco, les bandes du nord se subdivisaient, une partie envahissait les champs de Vera Cruz et la côte du Golfe, tandis que l'autre se répandait sur les Eitats de Campêche et de Yucatan. Il se détache parfois de la colonne principale, dans la région située au nord du Guatémala, des bandes qui se dirigent vers le Yucatan, entre les frontières de cet Fitat et de celui de Campêche, c'est-à-dire entre Halachó et Progreso, localité où les sauterelles apparaissent en premier lien.

Les invasions de la péninsule dı Yucatan durent généralement sept ans, bien que dans des circonstances que 1'on doit considérer comme exceptionnelles, il y ait eu des périodes de quatre, cinq ou six ans. Ces exceptions s'expliquent en admettant l'action ininterrompue de causes ennemies, qui affaiblissent et déciment les bandes ou bien en supposant que dans leurs migrations vers le nord-ouest, elles s'élèvent à de grandes hauteurs et quẹ, transportées par les vents, elles s'éloignent du continent et vont se précipiter dans les mers environnantes.

On a observé dans le Yucatan que les sauterelles, depuis les premiers sautillements de la larve jusqu'aux derniers vols de l'adulte, tendent à se diriger toujours vers l'est ou le nord-onest.

On a constaté que, dès qu'elles out poudu, les sauterelles meurent en grande quantité; celles qui survivent penvent avoir encore de vingt-huit à trente-deux jours de vie an plus. Bien des fois, celles qui ne nuenrent pas dans les champs s'élèvent à des hauteurs suffisantes, jusqu'à rencontrer un courant d'air, qui les entraîne vers le nord-ouest pour les précipiter dans la mer. 
Si le monnent de pondre n'est pas encore venu et que les bandes soient surprises pendant leur vol par l'arrivée de la nuit, elles continuent leur pérégrination toute la nuit, en se dirigeant toujours vers le nord-ouest. Au mois de mai I9I2, on observa pendant trois jours et trois nuits de suite des bandes qui, passant sur la ville d'Izamal (Yucatan), suivirent continuellement la même direction; beaucoup disparurent; on suppose qu'elles allèrent tomber dans les mers voisines.

Schistocerca peruviana. - Nous avons déjà eu l'occasion de dire que les apparitions de cette sauterelle au Péro 11 ne sont pas de date récente (début du XIX ${ }^{\text {̀̀me }}$ siècle), mais elles se sont manifestées d'une manière permanente, quoique avec des alternatives d'intensité et sur une étendue limitée, seulement deptis seize ans environ. Les accidents de terrain, qui abondent dans la zone infestée du Pérou, sont considérés comme la cause principale du peu d'étendue des terrains propices à la multiplication des sauterelles; de plus, il faut citer les variations atmosphériques fréquentes et brusques et l'action d'autres causes adverses naturelles.

Melanoplus spretus.-- Au Canada, il a sa région permanente c'est-à-dire son lieu originaire de reproduction où l'oul peut toujours le rencontrer en plus ou moins grande quantité, dans la partie méridionale de l'Alberta et dans la partie sud-occidentale du Saskatchewan. La région subpermanente, à savoir le territoire où la sauterelle peut se reproduire pendant un certain nombre d'années sauf à disparaître ensuite, est constituée par les deux régions contiguës du sud-est du Saskatchewan et du sud-onest du Manitoba. Le reste de cette province, jusqu'au Lake of the Woods, forme la région temporaire, c'est-à-dire le territoire que l'insecte ne visite que périodiquement et dont il disparaît d'ordinaire an bout d'un an.

De même qu'on le constate aux Etats-Unis, lorsque les sauterelles se sont extraordinairement développées dans la région permanente, l'insecte passe dans la région subpernuanente et de celle-ci dans la région temporaire, celle qui souffre le plus de la présence des sauterelles; dans la région subpermanente, les dommages sont plus fréquents que dans la région temporaie mais ils sont rarement aussi graves et aussi imprévus que dans cette dernière.

La même espèce tronve aux Etats-Unis sa région permanente dans les Etats de Montana, Wyoming et Colorado. La région subpermanente est constituée par le Dakota septentrional, le Dakota méridional et aussi par la partie occidentale du Kansas. La région temporaire se compose des Etats de 1'Union nord-anéricaine qui côtoient le Mississippi à 1'occident.

Emigrant de leurs foyers d'origine, les bandes du M. spretus arrivent le plus souvent, au commencement de l'été, dans le Dakota méridional; vers la moitié de l'été dans le Colorado, le Nebraska, le Minnesota, l'Iowa et dans la partie occidentale du Kansas ; elles arrivent à la fin de l'été dans la partie sud-est de ce dernier Fitat et dans le Missouri: elles ont été signalées dans le 'Texas vers la moitié d'octobre et même après. I a direction que les 
bandes prennent dans leurs migrations est donc, en général, celle du sud au sud-est; à l'ouest des Montagnes-Rocheuses, elles descendent vers les vallées et les plaines les plus fertiles, mais pas avec la même régularité que cellu dont elles font preuve dans 1'est.

Calliptamus italicus. - En Autriche, et plus exactement dans le Carso gorizien et en Dalmatie, on a constaté que l'invasion de cette sauterelle procède lentement de l'orient vers l'occident.

Nous avons déjà eu l'occasion de faire allusion à cette espèce pour ce qui concerne l'I talie et la Grèce, lorsque nous avons traité du Dociostaurus maroccanus dans ces deux pays.

Ėn I849, alors que l'apparition en masse des sauterelles fut signalée pour la première fois en S e r bi e, et précisément à.Korman, département de Kragujévac, on constata qu'elles venaient de l'est avec une grande rapidité. Malheureusement, on ne détermina pas alors de quelle espèce il s'agissait.

Les sauterelles existent en grande quantité dans tout le Sahara espagnol; ce n'est que lorsque le vent du sud règne, en automne, qu'il en tombe quelques-unes dans le Gouvernement politique-militaire de R i o-de-Oro .

La dernière bande de sauterelles observée au Togo dans le district d'Anecho (Ig03) se dirigeait, d'après ce que l'on rapporte, de l'orient vers l'occident. Dans le district de Lome, c'est en Igor ou en I903, au dire des indigènes, que les dernières bandes furent observées ; elles venaient du nordest. Les jeunes insectes, nés des œufs que ces bandes y avaient pondus, prirent leur route vers le sud-ouest; on affirme que dans le territoire de Awe les sauterelles seraient allées et venues encore une fois, après quelque temps, dans une direction contraire.

Quant aux sauterelles qui désolent 1'A ustralie, on peut dire, en général, que d'ordinaire elles se développent et se multiplient dans la partie intérieure, sèche de ce continent et que, dans des conditions climatiques favorables, elles augmentent rapidement et se présentent, à des intervalles irréguliers, réunies par millions.

Détruisant tout ce qu'elles trouvent sur leur passage, elles se dirigent généralement vers les districts côtiers, dès qu'elles ont atteint leur maturité. Parfois, d'immenses nuées passent d'un Jitat dans l'autre ; tel est, par exen1ple, le cas de Victoria où les satuterelles arrivent à travers la Notvelle-Galles du Sud. En général, les apparitions ont lien après nne on clenx an11ées de sécheresse.

CAUSES QUI DÉTERMINENT LA FORMATION DES BANDES, IES MIGRATIONS E'T LA MULTIPLICATION DES SAUTEREILES

On ne saurait affirmer actuellement, sans conrir le risque de tomber dans l'erreur, que l'on connaît avec certitude les causes qui induisent les sauterelles à se réunir d'abord, dès le premier âge, en bandes plus on moins 
imposantes et à voyager ensuite en parcourant des distances plus ou moins considérables. Ce qui paraît indiscutable à l'égard de ces animaux comme à celui d'autres, qui ont des mours analogues, c'est que tant leur rétnion que leurs migrations ont pour résultat final la conservation de l'individu et de l'espèce.

Mais, les causes premières de ces deux phénomènes distincts sont encore à trouver. Toutefois, de nombreuses conjectures ont été faites à ce propos ; nous allons mentionner les principales.

On voudrait admettre, par exemple, chez l'insecte l'existence de facultés naturelles, telles que l'instinct d'association et celui de migration; 1e besoin de protection mutuelle porterait les individus à se rassembler; la nécessité de chercher des conditions météorologiques favorables, de trouver la quantité de nourriture suffisante pour satisfaire les exigences d'un nombre extraordinaire d'individus, et, de plus, de fuir les causes ennemies naturelles déterminerait les déplacements et les migrations.

Parmi les causes qui favorisent la pullulation des sauterelles et en même temps les dommages qu'elles occasionnent à la végétation, on compte: la prédominance de conditions climatiques favorables à 1'éclosion et à l'évolution ultérieure des insectes, constituées, pour la majeure partie des espèces, par la succession d'années de grande sécheresse; 1'action des vents, des orages, etc., qui contribuent à la diffusion et à la dispersion des vols et, par conséquent, à la multiplicité des pontes; la diminution ou l'absence d'ennemis naturels; la nature aride, pierreuse du sol, particulièrement propice, en général, à la reproduction de ces orthoptères ; la négligence dans le défrichement des terres incultes où les œufs ont été pondus; l'emploi, dans certains pays, de systèmes culturaux déterminés, qui consistent à laisser en repos pendant un certain temps des étendues de terrain souvent considérables; 1'insuffisance ou l'absence d'une lutte systématique et générale, etc.

\section{NOURRITURE}

Dans leur premier âge, ces insectes se nourrissent, en général, des herbes tendres qui potissent dans les terres incultes, dans les prairies, dans les pâturages naturels, dans les lieux, en somme, où ces insectes sont nés, à moins qưils ne se soient développés directement sur des terrains cultivés.

Puis, au fur et à mesture que l'herbe vient à manquer dans les localités occupées d'abord et que ces orthoptères sont devenus plus voraces en se developpant, ils commencent à attaquer aussi les cultures; ils font souvent preuve d'une préférence plus ou moins marquée pour certaines d'entre elles.

Ainsi, des plantes cultivées, ce sont en général les céréales et les autres graminées qu’ils préfèrent, puis viennent les légumineuses à graine et à fourrage, les plantes potagères, les plantes fruitières, industrielles, médicinales, ornementales et, enfin, les essences forestières.

De plus, ils ont une prédilection pour les plantes à l'état vert, dont 
toutes les parties jeunes et tendres leur fournissent une nourriture convenable ; toutefois, ils paraissent accorder leur préférence aux feuilles bien qu'ils sachent tirer aussi un bon parti des tiges, des branches, des fruits, des graines et quelquefois même des racines.

Il est clair, d'autre part, que le choix et l'ordre de préférence des aliments sont susceptibles de varier, car ils dépendent de divers facteurs, parmi lesquels il faut compter les conditions physiques du pays infesté et son caractère botanique et agricole ; il faut tenir compte également à cet égard de l'espèce ou des espèces de sauterelles qui fréquentent les régions atteintes, et d'autres causes encore moins évidentes en apparence.

Même lorsque ce choix peut se faire relativement sur une vaste échelle, on observe parfois, chez ces insectes, des abstentions qui conduisent, dans certains pays, à une exclusivité d'alimentation. Il en est ainsi, en Chine, où, leur nourriture ne se composant que de graminées, ils laissent de côté les légumineuses (soya [Glycine Soja], Vicia, Pisum), les solanées (pomme de terre), le sésame; à Porto-Rico, à St.-Kitts et dans la Guy ane anglais e oì Schistocerca pallens n'attaque parmi les plantes cultivées que la canne à sucre; dans le Surinam, où les feuilles des jeunes Hevea souffrent seules de la présence d'une espèce de Schistocerca.

D'autre part, on a vu Dociostaurus maroccanus détruire les plantes potagères en $\mathrm{S}$ e r b i e, par exemple, et endommager fort peu les céréales qui se trouvaient dans leur voisinage immédiat, et cependant, les céréales, nous l'avons déjà dit, comptent parmi les plantes les plus recherchées par les sauterelles.

On a constaté aussi parfois qu'elles préféraient les plantes spontanées aux plantes cultivées. Ainsi, on peut dire, d'après les observations recueillies en Sardaigne sur D. maroccanus, que cette sauterelle n'envahit les cultures que lorsqu'elles se trouvent sur sa route, sans qu'il y ait dans leur voisinage des pâturages verts ; l'insecte attaque presque constamment les herbes spontanées qui infestent les cultures avant de s'adresser aux plantes cultivées. Les plus grands dommages sont constatés chez ces dernières là où il n' y a pas de prairies vertes ou lorsque ces dernières sont sur le point de mûrir. Si dans les champs de blé, d'orge, d'avoine, etc. les sauterelles ne trouvent pas suffisamment d'herbes à dévorer, elles attaquent et dévorent les céréales.

On a observé ailleurs aussi quelque chose de semblable: à Ch a c a chacare, petite île située non loin de la Trinité, S. paranensis préférait aux plantes cultivées quelques composés sauvages. Il faut noter toutefois à ce propos que cette même sauterelle a, dans la petite île voisine de $\mathrm{Patos}$, attaqué les cultures sans toucher à la végétation spontanée.

D'autre part, même parmi les plantes spontanées, ces orthoptères font un choix, en en mangeant un certain nombre, en en respectant d'autres, comme il résulte des observations et des renseignements recueillis en A 1 gérie au sujet du $D$. maroccamus et du S. tatarica.

Citons encore l'observation faite au Mexique, où S. amcricana, tout 
en attaquant de nombreuses plantes cultivées, fait preuve d'une préférence spéciale pour l'" icaban » (Croton albidus), euphorbiacée notoirement vénéneuse pour l'homme et pour le cheval.

De plus, une même espèce végétale peut aussi être diversement attaquée d'un pays à l'autre et parfois dans le même, par une unique espèce de sauterelles.

A cet égard, le comportement du D. maroccanus vis-à-vis de la vigne est digne d'être noté: celle-ci est plus ou moins gravement attaquée en I ta lie, en Bulgarie, en Algérie, etc. Par contre, l'insecte ne paraît pas y toucher en Hongrie. On raconte à ce propos que l'on vit en Igo6, à Nádudvar (Balassa-föld, ferme Meise), une bande considérable s'abattre sur un vignoble et y rester environ un quart d'heure, puis 1'abandonner intact, sans avoir entamé une seule feuille; pendant ce temps, une autre bande attaquait, non loin de la susdite ferme, une batteuse en plein mouvement, pour dévorer le blé presque entre les mains des ouvriers.

Tandis que le criquet marocain a parfois complètement dépouillé les oliviers de leurs feuilles dans plusieurs localités de la Grèce et de l'Algérie, ces arbres ne sont pas d'ordinaire soumis à ses attaques en Calabre (Cotrone).

D'après d'autres observations faites en I talie, cette espèce n'attaque jamais les tomates à Cotrone, tandis qu'en Sardaigne elles courent le risque d'être complètement détruites.

On a remarqué souvent en $\mathrm{A} 1 \mathrm{~g}$ érie que le blé, le maïs, le lin, le tabac, la vigne, le mûrier, les arbres fruitiers, etc. étaient respectés par $S$. tatarica, daus certaines localités tandis que dans d'autres ces plantes étaient considérablement endommagées par le même insecte.

Pendant sa dernière invasion au Mexique, S. americana infligea des pertes estimées à plusieurs millions de "pesos") (I) à la culture de 1'" henequén» (Agave rigida), le principal article d'exportation du Yucatan et du sud de la République et un des facteur les plus importants de la richesse de ces contrées; il n'en fut pas ainsi dans les invasions précédentes ou 1'"henequén" n'eut que peu à souffrir de cette même sauterelle.

De plus, une même plante est plus ou moins attaquée, non seulement d'après son âge - fait d'ailleurs explicable — mais même d'après ses conditions de culture : au Mexique, S. americana recherche peu les grandes plantes de caféier, mais en revanche il détruit les petites; dans le même pays, il ne mange pas le tabac écimé, mais si cette opération n'a pas été exécutée, il le dévore comme une vraie friandise.

Les choses changent presque totalement surtout lorsqu'il y a pénurie de plantes cultivées et de végétation en général. Alors, ces orthoptères ne sont plus aussi difficiles dans le choix de leur nourriture et ils dévorent presque tout ce qui se présente, y compris ce que dans d'autre conditions ils auraient laissé de côté. On les voit dans ces cas manger avec voracité toutes les parties d'un végétal, même ultramûr, sec et dur; ils arrivent même à ronger, avec le même acharnement que celui qu'ils auraient mis

(I) I "peso" d'or de soo "centavos" $=2,583$ fr au pair. 
à détruire des rejetons tendres et succulents, l'écorce et le bois du tronc et des grosses branches des arbres.

Au besoin, ils ne dédaignent pas des plantes très grossières, hispides ou épineuses ou coriaces, telles que Carlina, Galactites tomentosa, Cnicus, Echium, etc., en Sardaigne; Eryngium campestre, Atractylis gummifera, en Sicile; Carduns mutans, en Hongrie; les chardons aussi en Algérie; ou bien irritantes comme les euphorbes, en Sardaigne, Urtica urens, en Hongrie; ou bien ayant des propriétés notoirement vénéneuses comme, en Sardaigne, Thapsia garganica que les chèvres mêmes refusent ou comme, en A1gérie, l'harmel (Peganum Harmala), connu comme un poison par les indigènes et évité par les troupeaux; il en est de même pour les plantes à saveur âcre et à odeur désagréable comme la pomme épineuse (Datura Stramonium) en A1gérie et en Erythrée; d'ailleurs rien d'étonnant à cela, si on le rapproche de ce que nous avons déjà dit au sujet de l'"icaban" du Mexique. Rappelons ici encore les recherches expérimentales en cage entreprises à Constantine (Algérie) par J. KÜNCKer D'Herculais et Théophile Bauguil en I889, au sujet de la préservation des vignes contre les dommages causés par les sauterelles ; les expérimentateurs constatèrent entre autres résultats, que les solutions amères d'aloès, de gentiane, de Quassia amara, n'empêchaient pas les ailés de D. maroccanus d'attaquer les ceps; toutefois les deux auteurs précités ont estimé qu'il était permis de supposer que, à l'état libre, l'insecte aurait évité les vignes traitées aussi avec d'autres substances ayant des propriétés insecticides ou répulsives plus marquées.

Cependant, il existe- exception des plus rares - quelques plantes qute ces orthoptères respectent généralement. Telles sont, pour D. maroccanus, d'après ce que l'on rapporte de l'Espagne, le "diente de léon" (Taraxacum officinale, dent de lion, pissenlit); pour S. tatarica, dans l'I nde, le théier (on a constaté qu'il n'a subi que de très légers dommages, en I9r5, dans les districts de Darjeeling et de Terai et que, hors de 1'Inde, il n'a que très peu souffert à Cey $1 \mathrm{an}$, en I 895 , et qu'il n'est pas touché dans l'Union de l'Afrique du Sud), le "farash" ou Tamarix gallica (1'observation a été faite plus précisément dans le Radjpoutana) et Melia Azedarach ("bakain ", "Persian lilac ") qui, d'après ce que l'on a observé même très récemment à Djelfa, n'est pas plus touché en Algérie, où $S$. tatarica épargne aussi le caroubier.

Une autre sauterelle encore, $S$. paranensis, fait preuve en Argentine d'une profonde répugnance pour le $M$. Azedarach appelé dans ce pays-là "paraíso » et il évite même de s'y poser. Il en est presque de même en Urugua où le "paraíso " est épargué également, à moins que tout autre aliment vienne à manquer.

Dans l'Inde encore M. Azadirachta (" neem») est un autre arbre auquel Cyrtacanthacris succincta s'attaque rarement.

Ces insectes se nourrissent aussi de substances animales, soit dans les champs, soit dans les lieux habités où, à la recherche de nourriture, ils arrivent même à envahir les maisons jusque dans les recoins les plus cachés et y entament les denrées, les aliments déjà apprêtés, les meubles. Ėı 
A 1 gé rie, où $D$. maroccanus attaque les céréales aussi dans les magasins et n'épargne pas les pains de munition, les sauterelles ont parfois obligé les habitants à abandonner leurs demeures et à dormir en plein air.

On a constaté que les tissus eux-mêmes ne sont pas à 1'abri des attaques de ces insectes: au Japon, par exemple, on a vu Locusta migratoria dévorer en quelques instants des morceaux d'étoffe de laine aussi bien que du papier; en Algérie, des tissus de laine ou de soie ont été trouvés perforés ou lacérés par D. maroccamus; dans l'Union de $1^{\prime} \mathrm{A}$ frique du $\mathrm{S} u \mathrm{~d}$, Locusta pardalina a été jusqu'à faire quelquefois sa proie des vêtements mis à sécher; dans l'Uruguay on a vu $S$. paranensis manger de la toile.

De plus, ces or hoptères se dévorent voracement entre eux; ce sont les individus les plus faibles ou mutilés ou bien malades ou mourants qui sont attaqués de préférence. On sait d'ailleurs que Teltigonia albifrons pousse le cannibalisme à un tel point que les femelles ne se contentent pas de dévorer leurs mâles, mais qu'elles vont jusqu'à détacher leurs pattes postérieures, par autotomie, et les mangent.

Ceci posé, nous croyons utile d'indiquer ici quel est, dans chaque cas, le régime alimentaire de ces insectes; nous donnerons à ce propos tous les renseignements qu'il nous a été possible de recueillir dans les divers pays du monde. Les plantes dont ces orthoptères se nourrissent seront indiquées chaque fois dans l'ordre d'importance qu'elles paraissent avoir relativement aux insectes considérés. 
㶾

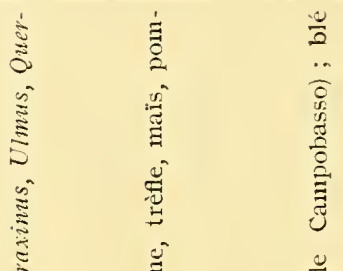

至

8

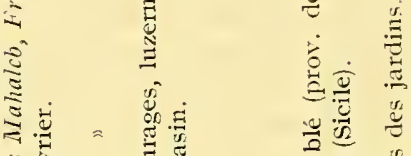

总

ट.

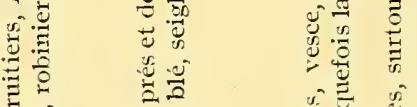

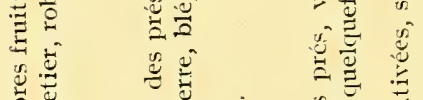

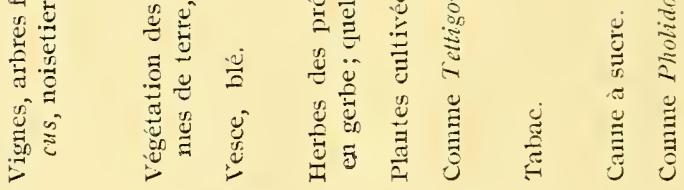

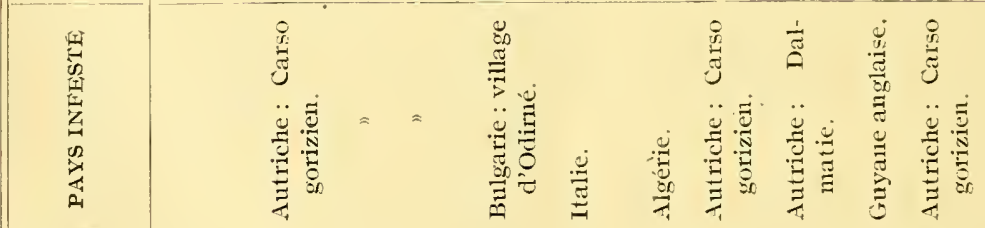

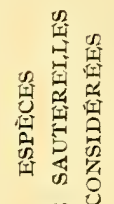

兵

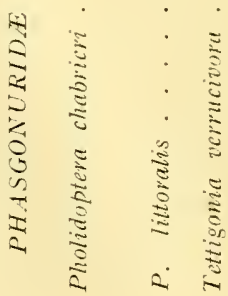
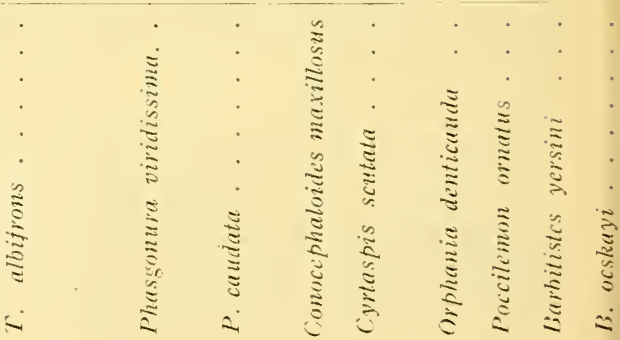

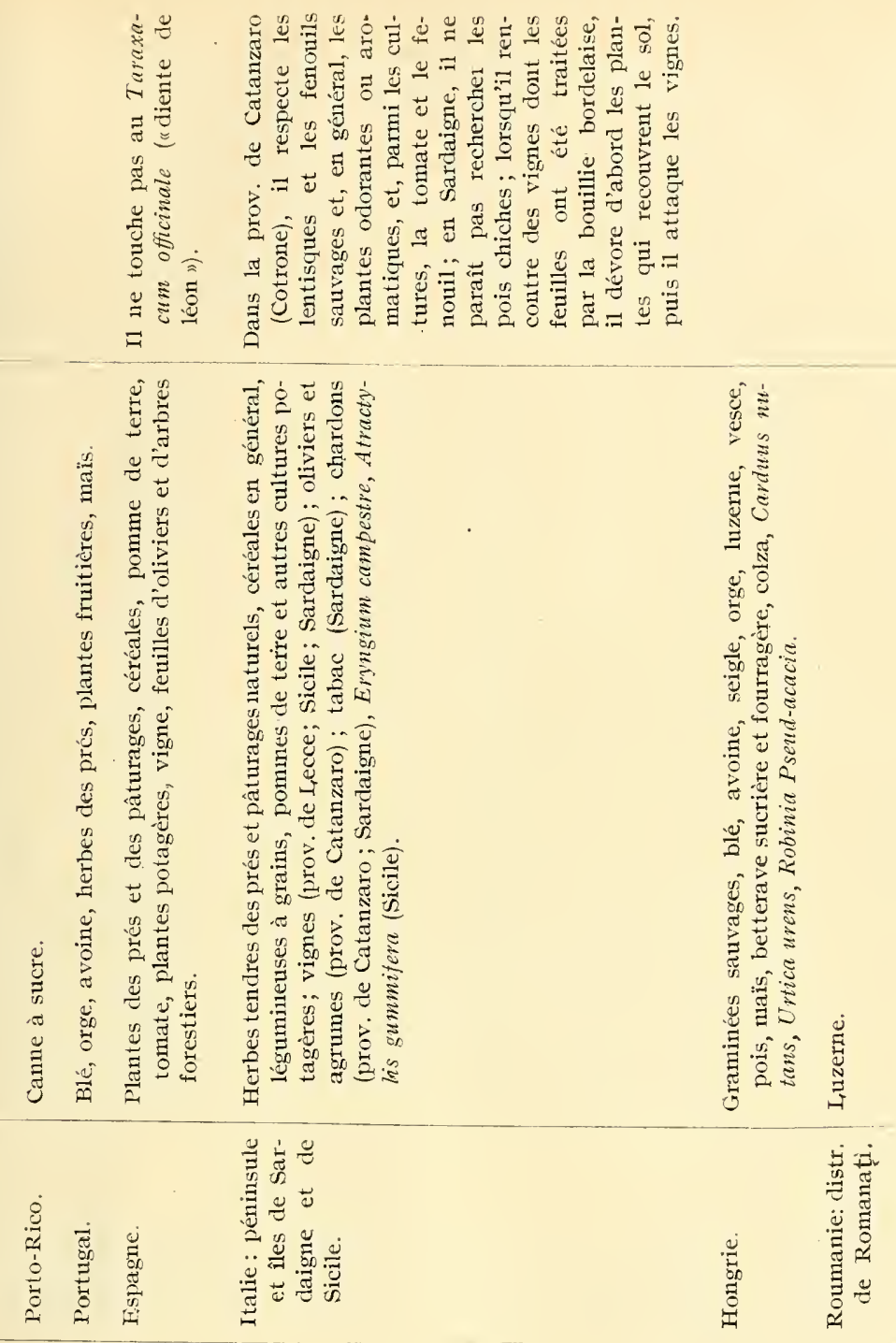

密

焉

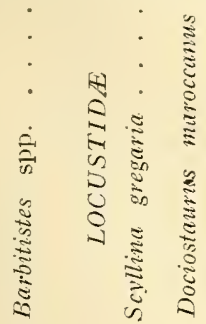




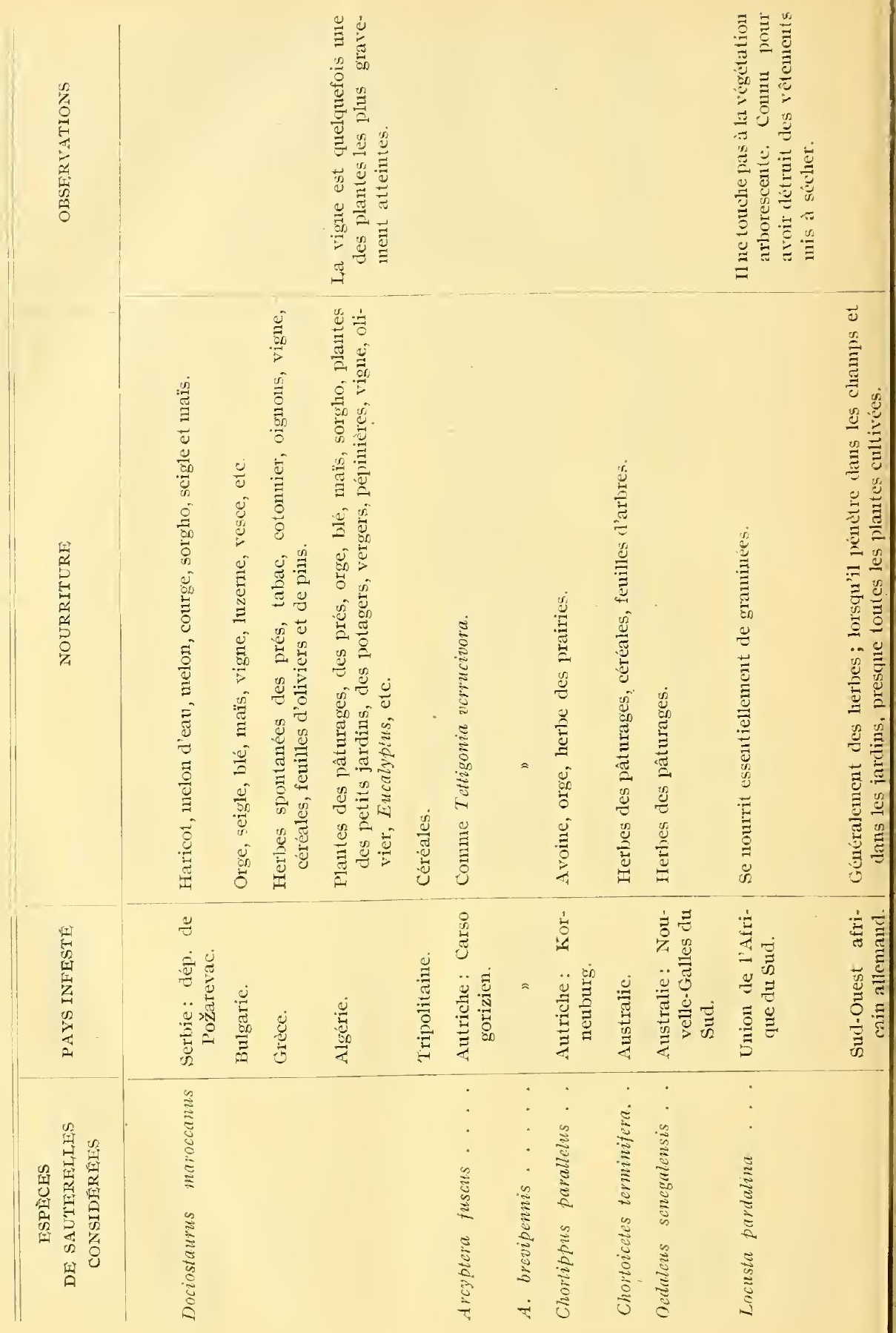




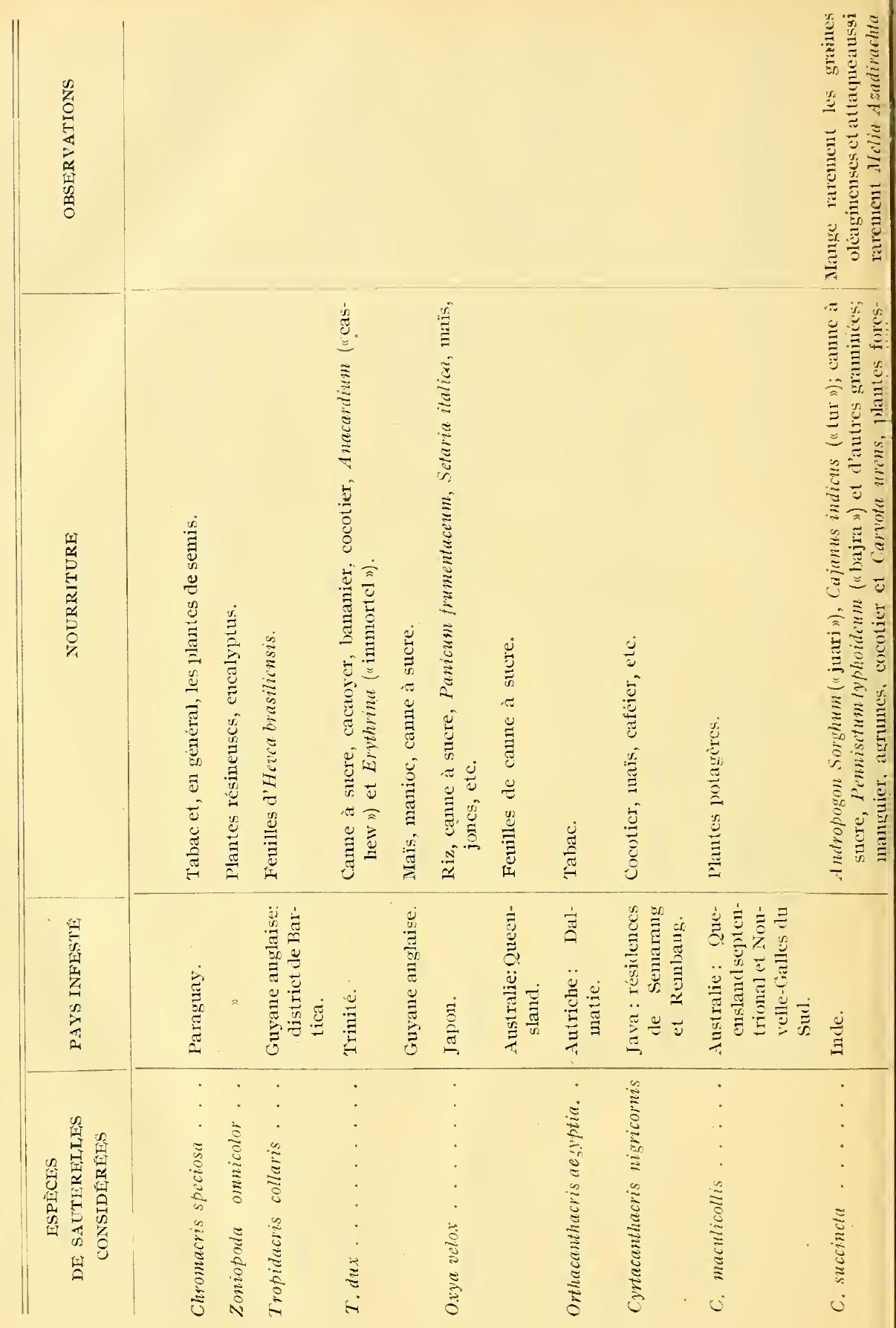




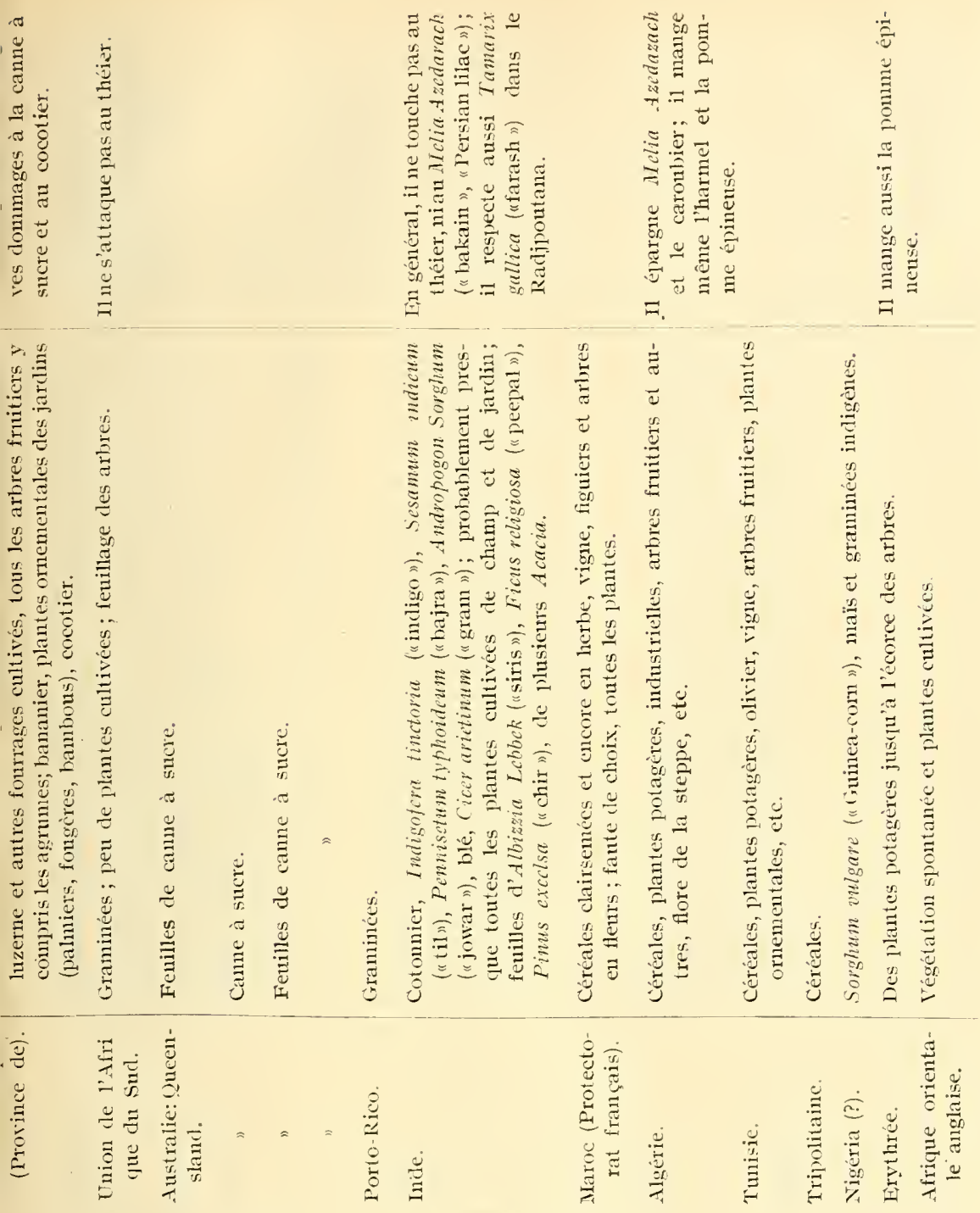




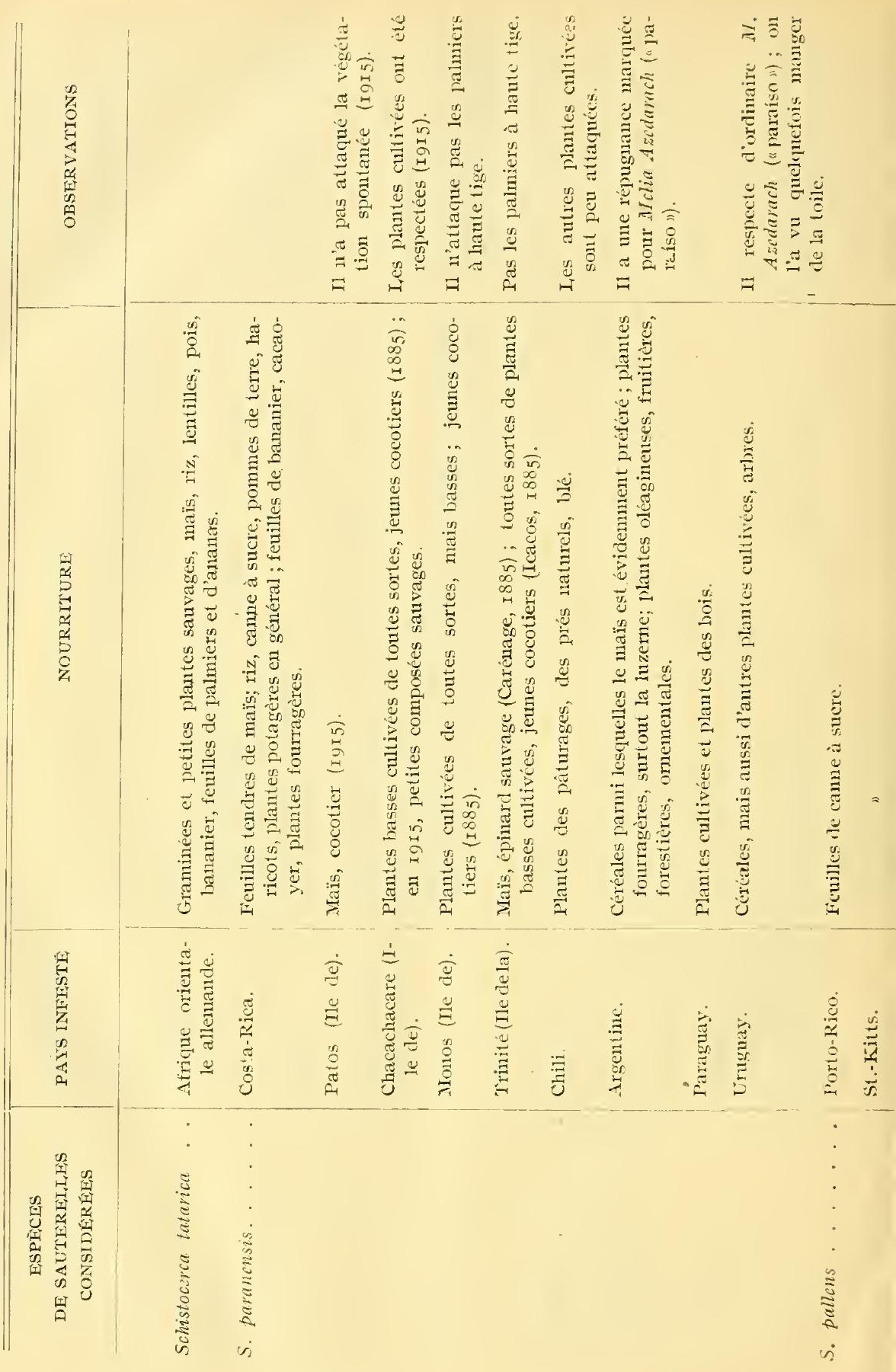




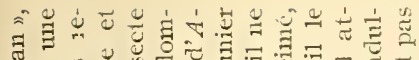

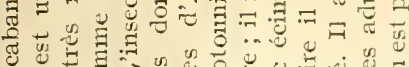

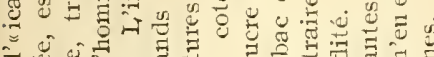

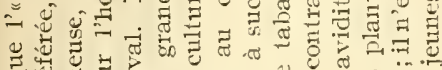

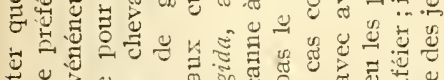

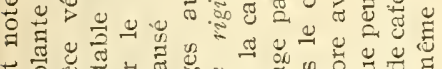
三

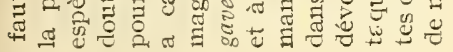
F

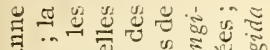
ฮี

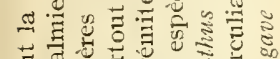

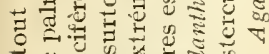

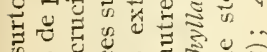

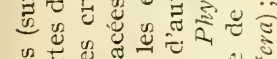

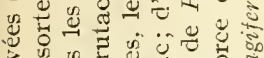

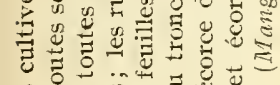

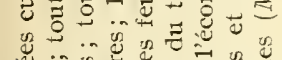

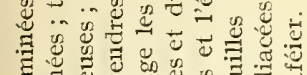

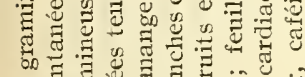

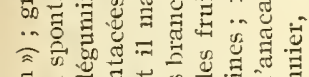

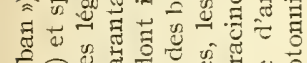
:

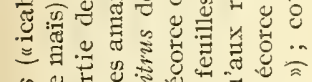

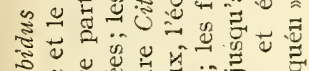

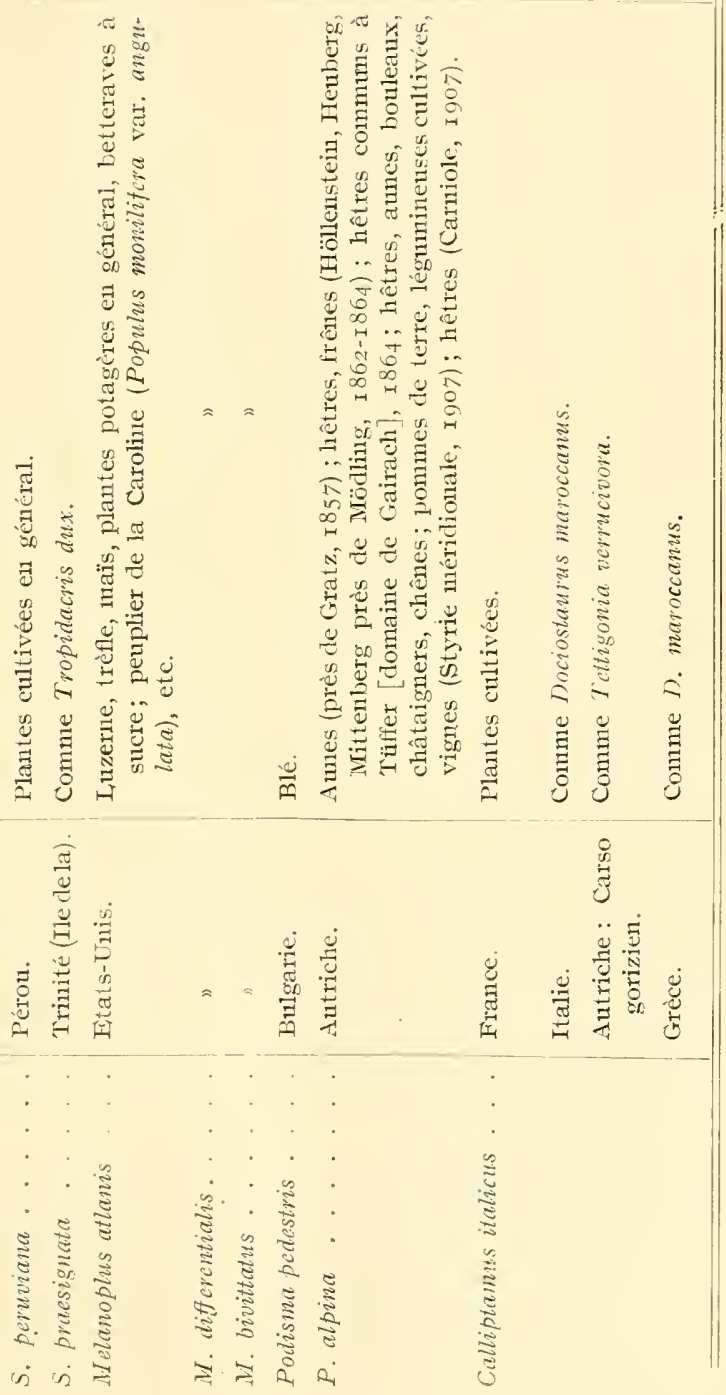



ORGANISATION FN VIGUEUR DANS LES DIVERS PAYS FN VUE DE LA LUT'TE CONTRE LES SAUTEREILES

\section{MESURES LÉGISLATIVES ET ADMINISTRATIVES *}

Il n'existe pas en Al1emagne de dispositions législatives concernant les sauterelles.

En France, par la circulaire du x6 avril I902, no 36 , le Ministre de 1'Agriculture a adressé aux professeurs départementaux et spéciaux d'agriculture des conseils et des renseignements en vue de prévenir, pour 1'année rgo2, les invasions de sauterelles dans les cultures de leurs régions. Cette circulaire donne spécialement des renseignements sur la biologie du Calliptamus italicus et sur les moyens de le combattre.

Le Portuga1 applique le règlement des Services pour la destruction des sauterelles, approuvé par décret du 20 février I902.

La loi du 2I mai I908, en vigueur en Espagne pour combattre et pour prévenir les fléaux des cultures, contient toutes les dispositions concernant l'organisation de la lutte contre les sauterelles. Toutes les mesures prises à cet égard par le "Ministerio de Fomento » s'inspirent précisément des dispositions de cette loi.

Les "Cọnsejos provinciales de Fomento " prennent de leur côté un petit nombre de mesures, mais celles-ci sont toujours conformes à la loi précitée, aux décrets royaux et à ceux que promulgue le "Ministerio de Fomento ».

Aux termes de cette 1oi, les propriétaires, agriculteurs, ingénieurs de toutes classes et leurs assistants, gendarmes, gardes 'champêtres, gardes assermentés, gardes forestiers et quelques autres du Service de garde et de surveillance rurale sont tenus, sous peine d'amende, d'informer la "Junta local de extinción de la langosta » de l'apparition des sauterelles et des dommages qui en résultent. Celle-ci en informe à son tour le "Comisario regio, presidente del Consejo provincial de Fomento ", ainsi que les chefs

* Pour plus de détails sur la plupart des mesures que nous rappellerons ici, consulter les publications suivantes éditées par 1'Institut international d'Agriculture:

Annuaire international de législation agricole. Rome, I912-1916, Ière-Vème année (I9I I1915), passim.

Le Service de prolection des plantes dans les divers pays. IIIème édit. Rome, I9I4, passim. 
des provinces limitrophes. Le "Comisario regio " charge un ingénieur agronome ou son assistant du constat sur place de ces dégâts; la "Junta " doit en outre avertir la circonscription municipale de l'apparition des sauterelles et du lieu et de la date de cette apparition.

Le "Comisario regio", de concert avec la "Junta " et le personnel technique, doit exiger des cultivateurs, dans la première quinzaine d'août, un rapport sur l'étendue des terres intestées, et, dans la seconde quinzaine du même mois, la "Junta " décide sur 1'opportunité d'établir un Service de surveillance sur place.

Le "Comisario regio " de la province envahie demande à l'autorité compétente d'interdire la chasse aux oiseaux insectivores, même lorsque la chasse est ouverte.

Si l'agriculteur ne prend pas directement part à la lutte contre les sauterelles, la "Junta » intervient, et exige pour 1'automne et 1'hiver 1'application de mesures opportunes én vue de la destruction de ces insectes.

Èn I t a 1 i e, la lutte contre les sauterelles s'est inspirée jusqu'à présent de la loi du I 5 juin I9II, no 529 , concernant les mesures en faveur des provinces envahies par le fléaut.

D'après cette loi, le Ministère de 1'Agriculture, de 1'Industrie et du Commerce est autorisé à nommer dans chaque province envahie un Commissaire temporaire, choisi de préférence dans le personnel technique dépendant de ce Ministère, et qui devra pourvoir à l'organisation et à la direction des travaux de destruction des sauterelles.

En Autriche, on n'a pas pris de dispositions concernant spécialement la lutte contre ces orthoptères.

Il n'y a pas, en effet, de lois de 1'Empire s'étendant, sur ce point, à tous les pays représentés au Parlement de Vienne.

I1 existe toutefois, pour les différentes parties de 1'Empire, des dispositions valables à cet égard et qui concernent la protection des cultures contre les chenilles, les hannetons et autres insectes nuisibles; bien que n'envisageant pas expressément les sauterelles, ces dispositions se prêtent cependant à une interprétation qui répond aux intentions du législateur et elles offrent un moyen légal également applicable à la destruction des sauterelles. Ainsi, par exemple, le paragraphe 2 de la loi pour la Bohême du 30 avril I870 impose formellement la destruction d'insectes nuisibles autres que ceux prévus dans le paragraphe I, si ces insectes apparaissent à n'importe quelle époque de l'année, dans les champs et dans les prairies, d'une manière à donner des préoccupations.

Des dispositions à peu près semblables accompagnent les lois analogues relatives à la Bucovine, à Gorizia et Gradisca, à 1'Istrie, à Trieste, à la Carinthie, à la Carniole, à la Moravie, à la Basse-Autriche, à Salzbourg, à la Silésie, à la Styrie, au Tyrol et au Vorarlberg.

Sauf les décrets promulgués de I870 à I880 et renfermant quelques dispositions concernant le Locusta migratoria, qui causa des dommages eu Hongrie au siècle dernier, la question des sauterelles n'avait jamais été réglée dans ce pays.

Elle n'avait même pas été explicitement envisagée par la loi XII 
de I894 sur l'agriculture et la police rurale; toutefois les articles 54 et $5^{8}$ de cette loi se prêtaient à être étendus aussi aux sauterelles.

C'est en effet d'après la teneur de ces dispositions que l'on entreprit les premiers travaux pour combattre le Dociostaurus maroccanus en I889-I893 et qu'on lutta, jusqu'en Igo6, contre l'invasion de I904, qui se prolongea jusqu'en Igo8.

Mais ces dispositions ayant été reconnues insuffisantes dès Igo6, la loi XXXI de I907, visant la destruction du criquet marocain, fut promulguée.

Voici comment la lutte est organisée en Hongrie : d'une part, les contrées menacées par le fléau sont placées, en vertu des articles I et 2 de $1 a$ loi XXXI de I907, sous le contrôle des communes et des propriétaires; d'autre part, un contrôle permanent est exercé par les experts de la Station nationale d'essais entomologiques. Ces fonctionnaires inspectent rigoureusement au moins une fois tous les trois ans le territorie entier de 1'A1föld (basse plaine de la Hongrie inférieure) et une fois au moins tous les deux ans toutes les contrées du pays où les sauterelles ont causé des dommages ainsi que celles où on les a rencontrées en nombre lors de la dernière inspection. Cet examen permanent permet de prendre en temps utile les dispositions préventives nécessaires. Dès qu'on s'aperçoit que la multiplication des insectes devient menaçante dans un endroit donné, on prépare pour l'année suivante tout ce qu'il faut pour conjurer le péril, pour limiter l'infestation à une surface tout à fait restreinte et pour détruire les sauterelles avec le moins de frais possibles. Sans doute, cette inspection occasionne quelques dépenses, mais, grâce à elle, le pays ne peut plus être surpris par une apparition soudaine des sauterelles, et celles-ci sont exterminées avant qu'elles n'aient pu causer de grands ravages.

La Roumanie, pour enrayer les dégâts des sauterelles et pour régler les dispositions à prendre en temps opportun, intervint à diverses reprises par des mesures législatives et administratives. C'est ainsi que les Chambres votèrent la loi du 26 mars I 860 pour la destruction des sauterelles. Cette loi fut complétée par le dispositif du I7 juin I860; un nouveau dispositif fut édicté le I7 juin I86r. Ceux-ci ont pour but principal de réglementer les moyens de destruction et de se procurer à temps la main-d'œuvre nécessaire. En effet, par le dispositif du I7 juin I860, chaque cultivateur est tenu de travailler pendant six jours pour combattre les sauterelles, et cela sur une étendue de $20 \mathrm{~km}$ autour de son habitation.

Les sauterelles n'ayant pas jusqu'ici sérieusement menacé la Se rbie, il n'existe pas dans ce pays d'organisation relative à la lutte contre ces insectes.

En Bulgarie, la lutte contre les sauterelles est réglementée par la loi sur la police des communes rurales, du I6 mars Igo5 (art. I85-I98), et par la loi du 7 avril Igro sur l'encouragement de la culture fruitière et de la viticulture.

Les préfets des départements prennent des mesures administratives d'après lesquelles les agriculteurs des localités envahies sont obligés de participer à la lutte contre les sauterelles. La population est aidée par l'armée. 
La direction de la lutte est confiée à 1'agronome de l'Ettat ou à ceux des chaires ambulantes d'agriculture.

Ė̀n Grèce, c'est actuellement la loi du 20 décembre I9I4-2 janvier I9I5, $n^{0}$ 5I2, relative à la destruction des campagnols et des sauterelles qui est en vigueur.

Nous ne trouvons pas, au Japon, de loi spéciale contre les sauterelles. Pour combattre 1'Oxya velox, les préfectures de Fou et de Ken établissent, aı moyen de décrets, les mesures à prendre conformément à 1a loi du 24 mars I896, $11^{\circ} \mathrm{I} 7$, relative aux moyens de prévenir les dommages des insectes nuisibles. Quant au Locusta migratoria, qui apparut, comme nous 1'avons déjà mentionné, dans la région de 1'Hokkaido en I880, les autorités locales publièrent, en cette circonstance, des réglements provisoires pour la destruction de cet orthoptère.

En Chine, la lutte contre les sauterelles est réglementée par le décret de l'empereur Jon-Tchien, promulgué le I7 août I723; par le décret de l'empereur Djélon, promulgué le Ig juillet I 754 et par sept articles de loi rédigés sous le même empereut.

Quant aux Etats Fédérés Ma1ais, des dispositions ayant rapport aux sauterelles se trouvaient déjà contenues dans l'acte législatif no I3 de I9I3 sur les fléatux de l'agriculture, publié le $I^{\text {er }}$ août de la dite année. Pour remédier aux lacunes des dispositions en vigueur constatées dans la pratique, on a approuvé ensuite et publié le I6 décembre I9I4, l'acte législatif $n^{0} 24$ de I9I4, amendant 1'acte précédent susmentionné

Les fonctionnaires chargés de diriger la lutte sont autorisés à entrer dans toutes les propriétés pour vérifier si les terrains sont infestés d'œufs ou de jeunes insectes; à appliquer, en cas d'affirmative, les mesures opportunes pour lenr destruction, en employant le poison (arsénite de sonde) ou des appareils mécaniques ou tout autre moyen. Si l'exécution des opérations relatives à la lutte est confiée à un fonctionnaire subalterne, ce dernier devra s'en tenir étroitement aux instructions écrites du fonctionnaire qui le délègue ; l'usage du poison est exclu, à moins que le fonctionnaire responsable ne décide qu'il peut être employé en toute sûreté.

Le propriétaire ou 1'occupant d'un terrain ou toute autre personne n'ont droit à aucune indemnité pour frais supportés ou dommages soufferts du fait de 1'application des dispositions en vigueur on d'autres mesures y relatives, à moins que 1e dommage ne soit causé par négligence, ou à dessein, ou sans un motif raisonnable. Les fonctionnaires chargés de la lutte sont done tenus de prendre les précantions nécessaires pour prévenir des accidents dont le bétail ou autres animaux domestiques pourraient être vistimes, lorsqu'on emploie le poison.

Le propriétaire ou l'occupant d'un terrain est tenu d'avertir par écrit le fonctionnaire inspecteur ou le bureau public le plus voisin de l'apparition éventuelle des santerelles, d'appliquer avec la plus grande diligence les moyens de destruction indiqués à cet effet par l'inspectenr, d'aider ce dernier dans les opérations relatives à la lutte, de mettre à sa disposition, sur sa demande et pendant une semaine seulement. la moitié dun person- 
nel placé sous la dépendance du propriétaire ou de l'oceupant du terrain infesté. Ce personnel sera rétribué sur les fonds publics; tous les autres frais supportés par le propriétaire ou l'occupant seront également remboursés si le fonctionnaire inspecteur les juge légitimes.

Les infractions des propriétaires ou occupants aux dispositions des mesures en vigueur sont punies d'une amende qui ne peut pas dépasser 250 dollars (735 fr).

Le Gouvernement de l'I n d e a transmis constamment des ordres et des instructions détaillées à ses fonctionnaires au sujet des mesures à prendre vis-à-vis de l'apparition des sauterelles. Lorsqu' on craint leur invasion, des officiers spéciatix des revenus, aidés d'un nombre convenable de fonctionnaires subalternes, sont détachés pour entreprendre la lutte, à laquelle ils procèdent avec la collaboration des Conseils de district et d'autres agents locaux.

Dans l'île de Chy p r e se trouve en vigueur, en ce qui concerne les mesures à suivre pour les opérations relatives à la destruction des sauterelles, la loi XI, en date du 2 juillet I88I.

Ein ce qui concerne le M a r o c (Protectorat français), où la création des Services de 1'Agriculture est de date très récente, le Chef de ces Services a rédigé et publié, au commencement de I9I5, en vue d'une invasion éventuelle et d'après les observations faites en Tunisie, une notice générale sur les moyens de défense contre les sauterelles. Cette notice contient aussi les mestres administratives proposées pour être adoptées au besoin, concernant l'organisation et la direction de la lutte dans chaque subdivision territoriale, le droit de réquisition de la main-d'œuvre et des animaux, l'acquisition du matériel à emplayer, les mesures d'hygiène à prendre pour protéger les sources et les points d'eau contre la pollution par les sauterelles et les criquets (insectes non ailés).

En A $1 \mathrm{~g}$ é ri e, des dispositions sont prises au fur et à mesure des besoins. Comme il résulte de la circulaire du Gouverneur général, en date du 5 février I9I5, le Service du phylloxéra, qui porte actuellement le nom d'Inspection de la défense des cultures, prête dorénavant son concours à la lutte contre les santerelles. Par circulaire du II décembre I9I5 le Gouverneur général a adressé aux autorités dépendantes les instructions générales pour la lutte contre ces insectes en prévision d'une nouvelle invasion.

Le I4 février Igr6 est entré en vigueur en Tunisie le décret qui, abrogeant les dispositions du décret du 7 mai $189 \mathrm{I}$ relatif à la destruction des sauterelles, prend des mesures nouvelles pour la destruction des sauterelles, des pontes et des criquets.

Toute personne, non empêchée par des motifs légitimes, est tenue de prêter son concours à la défense contre les sauterelles, pontes et criquets et d'obtempérer aux réquisitions de personnel, d'animaux, de matériel et de produits nécessaires à la destruction. La main-d'œuvre réquisitionnée doit être fournie gratuitement. En dehors du cas prév11 dains l'article 2, les services d'animatix, de matériel et de fournitures de produits donnent 
droit à une indemnité dont le montant est fixé dans chaque circonscription par le Contrôleur civil ou Chef de Bureau de renseignements.

Les propriétaires et les occupants à $11 n$ titre quelconque d'inmmeubles infestés de sauterelles, pontes ou criquets, devront en faire la déclaration aux autorités locales, exécuter sans délai les travaux de destruction avec les animaux et le matériel dont ils disposent, se procurer tous produits nécessaires, employer à la défense une main-d'ouvre, en rapport avec la superficie des propriétés, qui ne peut être inférieure à : I homme pour 50 ha de parcours; I homme pour Io ha de céréales, oliviers ou jachère; I homme pour 5 ha de vignoble ou culture sarclée; I homme pour I ha de jardin ou verger. Ils doivent, enfin, ouvrir l'accès de leur propriété au personnel du Service de la défense et se conformer aux mesures prescrites par les autorités locales ou par les délégués du Directeur général de1'Agriculture. L,es services prêtés par les propriétaires ou occupants sur leur propre fonds ou exigés d'eux sont à la charge des intéressés.

Dans le cas où les propriétaires ou occupants ne se conformeraient pas aux dispositions précédentes, il sera procédé d’office, par les soins de 1'autorité administrative, aux travaux de destruction, et cela aux frais des intéressés, sans préjudice des pénalités encourues: amende de 50 à Iooo fr et 5 à 30 jours de prison on 1'une de ces deux peines seulement; les articles 463 du code français et 53 du code tunisien sont applicables.

En Egypte, trois décrets établissent le régime de la lutte contre les sauterelles : celui du I6 juin I8gI relatif aux réquisitions pour la destruction de ces insectes; le décret dı 26 avril Ig04, qui impose le labourage ou le sarclage des endroits où les sauterelles ont pondu leurs oufs, et le décret du IS mai I9I5 modifiant en partie celui du I6 juin ISgI.

De plus, le Ministre de 1'Agriculture a envoyé, en I9I5, aux autorités placées sous ses ordres plusieurs circulaires visant la destruction des sauterelles. A rappeler particulièrement la circulaire du 25 février, $n^{\circ} 63$, dans laquelle il a réuni toutes les instructions relatives à la lutte, en résumé de celles données précédemment par les Ministères de 1'Intérieur et de 1'Agriculture.

En Erythrée, les sauterelles sont visées par 1'art. I28 du règlement pour les Conmissariats et les Résidences; par la circulaire du Bureau de colonisation du 9 septembre I905; par le décret du Gouverneur du I5 novembre de la même année; par la circulaire du 3 août IgIf et enfin par les édits que les Commissariats promulguent chaque année.

Aucune disposition n'a été prise jusqu'à présent dans la Nigéria.

I a lutte contre les sauterelles a été 1'objet de deux circulaires du Gouverneur général du Congo belge; l'une, en date du I2 novembre ISg6, contient des instructions générales pour combattre les sauterelles en cas de passage de ces insectes; 1'autre, émise le 4 juiln Igo\%, se rapporte à la destruction des sauterelles à l'aide de l'arsénite de soude.

Pour lutter contre ces insectes, l'Afrique orientale allemande, la Province de Mozambique, la Rhodésia méridionale et le $\mathrm{Sud}$-Onest africain allewand possèdent nue organisation qui correspond parfaitement - on en aura bientôt l'explication — à celle 
créée, il y a quelques années, dans 1'Union de l'Afrique du Sud qui comprend, comme on le sait, les provinces du Cap de Bonne-Espérance, du Natal, du Transvaal et de 1'Etat libre d'Orange.

L'opportunité d'une coopération ou d'une action combinée, dans la lutte contre le fléau des sauterelles qui se renouvelait chaque année, avait été reconnue depuis longtemps et à plusieurs reprises par les diverses Administrations de 1'Afrique du Sud. Le 7 décembre I904, le Gouvernement du Natal, qui avait toujours déployé daus ce sens une activité spéciale, présenta une demande aux Gouvernements de la Colonie du Cap, du Transvaal, de la Colonie du flenve Orange, du Bassoutoland et ensuite à celui aussi de Mozambique, pour savoir s'ils étaient prêts à collaborer avec lui dans l'œuvre de destruction des sauterelles.

Cette initiative fut favorablement accueillie, surtout par le T'ransvaal où, selon la méthode adoptée au Natal, une campagne énergique fut entreprise, au début de I905, sous la direction de l'entomologiste du Gouvernement, M. C.-B. Simpson, qui eut 1'idée de créer immédiatement une organisation centrale pour la lutte contre les sauterelles.

M. Simpson soumit son idée au comte de SELBORNE, Haut-Commissaire de Sa Majesté Britannique pour le Sud-Afrique, qui en comprit aussitôt toute la valeur pratique. Celui-ci adressa, le $I^{\text {er }}$ juin Igo6, aux Gouverneurs de la Colonie du Cap, du Natal, de la Colonie du fleuve Orange et à 1'Administrateur de la Rhodésia méridionale une Note contenant la première proposition officielle pour l'institution d'un Bureau central en même temps qu'un projet des règles d'après lesquelles le Bureau aurait dû exercer ses fonctions, et conseillant aussi de réunir une Conférence des représentants des diverses Colonies pour discuter ce projet.

La Conférence eut lieu, en effet, à la suite de ces pourparlers ; elle fut inaugurée par le comte de SELBORNE lui-même, et tint ses séances le 20 et le 2 I août Igo6 à Prétoria, capitale du Transvaal. I,es représentants de la Colonie du Cap, du Natal, du Transvaal, de la Colonie du fleuve Orange et du Bassoutoland y prirent part. La première des treize décisions adoptées par cette İ̀re Conférence intercoloniale contre les sauterelles recommandait l'institution immédiate d'un Bureau central, dont l'existence devait être assurée pendant cinq ans au moins, et qui serait chargé de recueillir é d'enregistrer dans des tableaux les mouvements des sauterelles à travers toute 1'Afrique britannique du Sud et de distribuer des rapports à ce sujet.

L'organisation centrale que l'on souhaitait se trouvait donc, dès lors, un fait accompli : 1e "South African Central Locust Bureau » s'installait à Prétoria. Les frais étaient couverts par des contributions de fonds au prorata versées par les diverses possessions britanniques de 1'Afrique du Sud, à savoir la Colonie du Cap, le Natal, le 'Transvaal et la Colonie du fleuve Orange - compris actuellement dans 1'Union de l'Afrique du Sud - la Rhodésia méridionale, le protectorat du Bechuanaland, 1e Bassoutoland, le Souaziland et, en outre, par le Sud-Onest africain allemand et par la Province portugaise de Mozambique.

On reconnut plus tard que l'apparition des sauterelles dans les pays 
susmentionnés pouvait avoir une certaine relation avec l'apparition de ces insectes dans les régions limitrophes situées au nord; le Bureau fit aussitôt des démarches afin d'obtenir au moins des rapports annuels sur les conditions d'existence des sauterelles dans les territoires des Gouvernements de 1'Afrique orientale allemande, du Nyassaland, de la Rhodésia du nordest et de la Rhodésia du nord-ouest; ces deux dernières sont maintenant réunies saus le nom de Rhodésia septentrionale.

L'entomologiste du Gouvernement du Transvaal, entouré de quelques aides, prit, en qualité de secrétaire de l'institution, la direction immédiate du Bureau; et le Bureau fut, à son tour, placé sous la dépendance d'un Comité de contrôle composé d'un représentant de chacune des Administrations contribuantes. Ce Comité, à qui furent confiées la direction et la surveillance des affaires et des propriétés du Bureau, devait tenir nne assemblée générale au moins une fois par an, à une époque et dans une localité à déterminer chaque fois.

D’autre part, les Gouvernements intéressés à l'entretien du Bureau eurent soin d'organiser chez eux, s'il n’existait pas déjà, une Service spécial chargé de recueillir les renseignements et les avertissements relatifs au développement et au mouvement des sauterelles dans leurs territoires respectifs.

Pour signaler la présence des sauterelles, on a généralement adopté le système des cartes postales. On lit, imprimé sur un côté, un formulaire qui indique, sauf modifications et adjonctions selon les pays, la date de l'apparition des sauterelles, la localité où elles ont été signalées, l'espèce à laquelle elles appartiennent, le stade de 1'insecte (ailé ou non ailé), la direction prise, puis des observations variées, le nom et la résidence de l'observateur. I, 'autre côté porte l'adresse dı bureau ou de l'autorité locale à qui doivent être renvoyées les cartes postales dûment remplies. On a même parfois prescrit d'expédier au bureau compétent quelques exemplaires des sauterelles observées dans la région.

Ces formulaires imprimés ont été distribués dès le début, dans chacun des pays, aux fonctionnaires chargés de signaler la présence des sauterelles et d'organiser la lutte contre ces insectes, ainsi qu'aux particuliers disposés à prêter leur concours.

Après avoir groupé tous les renseignements provenant des différentes parties de son territoire, 1'Administration devait les transmettre sans délai au secrétaire du Bureau de Prétoria. Là, on reportait ces divers renseignements au moyen de signes conventionnels sur des cartes géographiques, et le secrétaire, mois par mois, expédiait le plus vite possible à tontes les Administrations nue petite carte des régions de 1'Afrique situées au sud du ro ${ }^{\circ}$ de latitude sud et sur laquelle se tronvait marquée, de manière à 1'apercevoir au premier coup d'neil, la position des sauterelles sur tous les territoires intéressés ; chaque pays pouvait facilement ainsi adopter les mesures qu'exigeaient les circonstances.

D'autre part, chaque Administration eut soin de créer sur divers points de son propre territoire des dépôts de nuatières insecticides et d'ontils appropriés, afin de ponvoir, à l'occasion, entreprendre innnédiatennent les opérations de délense. I,insecticide à employer et les outils nécessaires 
pour s'en servir étaient distribués aux particuliers, souvent gratuitement, ou au prix d'achat, ou, s'il s'agissait d'appareils, même sous forme de prêt.

Les réunions annuelles du Comité de contrôle du Bureau furent tenues successivement, de I907 à I9Io, à Prétoria, à Durban (Nata1), au Cap et à Bloemfontein (Colonie du fleuve Orange).

I,es quatre rapports annuels préétablis dı Comité de contrôle témoignent de l'action que le Bureau a exercée de concert avec les pays adhérents et font aussi çonnaître les travaux effectués parle Comité pendant ces années.

Lorsque les Colonies les plus directement intéressées à l'existence du Bureau se groupèrent pour constituer 1'Union de l'Afrique du Sud, la raison principale qui avait déterminé la création de ce même Bureau tomba d'elle-même. Néanmoins des accords furent pris entre ces divers pays pour obtenir que la Division d'Entomologie de Prétoria, placée sous les ordres du Département de 1'Agriculture de 1'Union, assumât et continuât - comme cela a réellement lieu — les fonctions précédemment remplies par le Burea1.

Dans l'Afrique orientale allemande - où, comme on l'adit, il y a actuellement une organisation correspondant à celle déjà adoptée dans les pays du sud de l'Afrique - il est prescrit normalement que les apparitions de sauterelles doivent être signalées, par cartes postales, au moins une fois l'an, en janvier, à l'Institut impérial d'Amani, à moins qu'il ne s'agisse de bandes importantes : dans ce cas, les renseignements doivent être immédiatement communiqués, et 1'on doit expédier en même temps quelques exemplaires des sauterelles en question, afin que l'on puisse déterininer exactement l'espèce dont il s'agit.

Dans le dernier cas - à savoir lorsque des bandes conśidérables de santerelles migratrices apparaissent isolément, ou bien si une véritable invasion se manifeste en quelque localité - après que la première apparition a été signalée, l'envo: de cartes postales ou de rapports plus détaillés est exigé mois par mois jusqu'à ce que les sauterelles aient de nouveau complètement disparu.

L'Institut d'Amani transmet annuellement à Prétoria un réstumé des renseignements reçus.

De plus, près de cinquante localités de la Colonie possèdent des dépôts de l'insecticide choisi pour la lutte.

Les mesures adoptées dans la Province de Mozambique sont: l'ordonnance du Gouverneur général, no 864, du I5 novembre Igo6, relative à l'envoi par cartes postales ou par télégramme, au Secrétariat général du Gouvernement, des renseignements concernant l'apparition des sauterelles et la manière dont elles se comportent dans la Province; l'ordonnance du Gouverneur général, n10 4I9, du 22 mai Igo8, imposant entre autres le concours de leur main-d'œutre, dans la lutte contre les sauterelles, à tous les habitants de la Province résidant hots des villes ou villages chefslieux de districts, quand l'ordre leur en est donné par les autorités locales. Les infractions sont punies d'une amende de 25 à 50 "milteis " (I 40 à 270 fr) out de I à 6 mois de prison. 
Un module spécial imprimé, contenant le même formulaire que les cartes postales, a été adopté le $\mathrm{I}^{\mathrm{er}}$ août IgI 4 pour le rapport mensuel sur les mouvements des sauterelles dans la Province.

A11x sièges de circonscriptions et de capitaineries des districts de Lourenço-Marques, Inhambane, Téte et Mozambique, le Gouvernement entretient, par l'entremise de la Section d'Entomologie du Département de 1'Agriculture, un stock des outils et de 1'insecticide nécessaires pour la lutte.

Le Gouvernement du Mozambique et la Division d'Fntomologie de Prétoria ont pris des accords pour continuer l'échange des renseignements concernant l'apparition des sauterelles, comme cela avait lieu avec le "South African Central Locust Bureau ».

Aucune loi sur la destruction des sauterelles n'est en vigueur dans la Rhodésia méridionale, mais 1'Administration pourvoit à la lutte et vient en aide aux agriculteurs en leur distribuant gratuitement le matériel nécessaire.

L'exécution des travaux de lutte est confiée dans tous les districts à des fonctionnaires spéciaux choisis parmi les membres de la Police britannique de 1'Afrique du Sud; cenx-ci ont sous leur dépendance des indigènes chargés des travaux manuels. En outre, on met du matériel à la disposition des Commissaires indigènes qui penvent faire beaucoup avec l'aide des indigènes. Les chemins de fer de la Rhodésia coopèrent eux aussi à la lutte et 1'Administration leur fournit le matériel dont ils ont besoin. Des feuilles contenant les instructions sur les méthodes à employer pour assurer l'efficacité de la l11tte sont données gratuitement à tous les intéressés.

I Les cartes postales pour avertissements sont distribuées à la Police, aux Commissaires indigènes et à tous ceux qui offrent leurs services; une fois remplies, elles sont adressées au Département de 1'Agriculture à Salisbury, d'où les renseignements recueillis étaient auparavant transmis, tous les mois, au Bureau de Prétoria.

Ap1ès la disparition de ce dernier, des accords ont été pris en vile d'échanger chaque mois des renseignements entre la Rhodésia méridionale et le Département de 1'Agriculture de 1'Union, et de signaler telégraphiquement 1'approche de vols de sauterelles vers les frontières.

Les Bureaux d'arrondissements et de districts et les postes de Police sont chargés de l'exécution des travaux pour la destruction des sauterelles dans le Sud-Ouest africain a11emand.

L'organisation y est à peu près semblable à celle des autres Colonies de l'Afrique du Sud.

En Igog, on comptait dans le territoire de cette possession allemande jusqu'à cinquànte-sept dépôts d'appareils et d'insecticide.

Dans 1'Union de 1'Afrique du Sud, lorsque le Burean cessa de fonctionner, on promulgua, le I5 avril I9II, la loi destinée à prévenir dans le territoire de 1'Union l'introduction et la propagation d'insectes et autres invertébrés nuisibles à l'agriculture, de maladies des plantes, de maladies des abeilles, et à réglententer 1'importation d'animanx exotiques.

Le deuxième chapitre de cette loi envisage la destruction des satterel- 
les. Les occupants de terrains sont tenus d'informer aul plus tôt l'autorité la plus proche, soit par écrit, soit d'une autre manière, de toute observation d'œufs de sauterelles et de toute apparition de jeunes individus (" voetgangers ») de ces insectes.

L'autorité qui a reçu cet avis en transmettra la teneur au Département de 1'Agriculture qui peut, au moyen de ses fonctionnaires, prendre les mesures opportunes.

L'occupant doit procéder immédiatement aux travaux de destruction après avoir consulté le Département; le matériel nécessaire pour cette destruction lui sera fourni gratuitement par le Département. Chasser les " voetgangers" vers le terrain de son voisin est un délit, l'occupant doit prouver d'une façon satisfaisante qu'il a pris toutes les mesures possibles pour les détruire et qu'il ne les a pas chassés vers le terrain de son voisin.

Fin cas d'inexécution, sans raisons valables, des dispositions de la loi, le Département devra être remboursé, selon les modalités que l’on jugera à propos, des frais supportés pour les opérations de lutte.

Si le terrain infesté fait partie d'une réserve ou d'une concession indigène et qu'un habitant de ce terrain n'exécute pas, sans motifs acceptables, la partie raisonnable du travail qui lui incombe, il sera puni d'une amende qui ne pourra pas dépasser 5 livres sterling (I 6 fr environ), ou, si le payement n'est pas effectué, d'un emprisonnement de quatorze jours au maximum.

Dans l'éventualité d'invasions futures, les pouvoirs administratifs ont été dévolus, comme on 1'a déjà dit, à la Division d'Fntomologie du Département de 1'Agriculture de 1'Union.

Le Canada et les Etats-Unis n'ont jamais pris de mesures législatives se rapportant particulièrement à la lutte contre les sauterelles; on n'y compte pas non plus d'organisations créées ou existant à cet effet.

Le Gouvernement de Costa-Rica a promulgué le décret no 40, en date du 8 juillet I9I5, portant réglementation de la campagne contre les sauterelles et abrogeant, en conséquence, les décrets $n^{0}$ I 6 et $n^{0} 23$ du 3 et du If juin de la même année.

En I9I5, le Gouvernement a organisé militairement, sur certains points du territoire, cinq ou six cents hommes destinés aux travaux de destruction.

A la Trinité, en vertu des dispositions de l'ordonnance relative à la protection des plantes, les inspecteurs nommés par le Gouverneur ont la faculté d'accéder dans les terrains ou dans tous autres lieux pour constater la présence ou l'absence des sauterelles et de prescrire les mesures de défense opportunes; si ces prescriptions restent lettre morte, les opérations sont exécutées d’office aux frais de l'intéressé, sauf décision contraire du Gouverneur. 
I1 n'existe aucune mesure légale pour les autres îles du groupe des petites Antilles.

On ne trouve pas davantage au Pér ou de mesures législatives réglementant l'action de l'Etat ou celle des particuliers dans la lutte contre les sauterelles; il n'y a pas non plus d'organisations locales dans ce même but.

Pour ce qui est du Brési1, l’Etat de São Paulo passa, en IgIo, un contrat avec M. CAsirno Boy, membre de la "Defensa Agrícola " de 1'Argentine, pour un cours théorique et pratique sur les procédés de lutte contre les sauterelles. Plus tard, en IgII, le Ministre de l'Agriculture attacha à son service ce même fonctionnaire, qui avait donné cet enseignement dans 1'Etat de Rio Grande do Sul et qui était passé ensuite dans celui de Rio de Janeiro, afin que le personnel de la "Defesa Agricola » brésilienne et les élèves de 1'“ Aprendizado Agricola » de Horto da Penha puissent profiter de ses leçons.

La "Defesa Agricola » du Ministère de l'Agriculture dispose de dépôts spéciaux pourvus du matériel nécessaire à la lutte.

En date du 8 décembre I9I5, le "Prefeito municipal » de Agudos a publié une loi rendant obligatoire la destruction des saterelles sur le territoire de la municipalité.

La loi $n^{0} 3708$, qui réglemente actuellement la destruction de la "langosta " (Schistocerca paranensis) sur tout le territoire de la République Argentine, a été sanctionnée le 22 septembre I898.

Les habitants de la République âgés de quinze à cinquante ans - sauf ceux qui en sont physiquement empêchés, 1es femmes, les ecclésiastiques, les employés de 1'Ettat et des provinces astreints à un service journalier, ceux qui dépendent des entreprises de chemins de fer, hors du terrain de ces dernières - sont tenus de prêter, contre rémunération, leur concours personnel pour la destruction de la "langosta " et de fournir les outils et les animaux nécessaires, sauf les animaux destinés à l'amélioration de la race.

Cette obligation incombe aux habitants dans le territoire compris dans un rayon de ro $\mathrm{km}$ de leur domicile respectif et dans la juridiction de la Commission locale chargée de surveiller le respect de la loi et, pour ceus qui habitent dans les centres urbains, elle s'étend jusqu'à ro $\mathrm{km}$ en dehors de l'enceinte de la ville.

L'obligation ne peut pas dépasser vingt jours de travail consécutifs ou trente jours de travail alterné ; on peut en être exonéré moyennant le payement de 2 "pesos" (4,40 fr) par jour de travail ou bien en livrant, d'accord avec la Commission du district, une certaine quantité d'adultes, de larves ou d'œufs ramassés dans les limites fixées par la Commission centrale.

Sauf les personnes qui en sont empêchées physiquement, les femmes, les ecclésiastiques et les adultes au-dessus de soixante ans, tous les propriétaires ou occupants, toutes les entreprises de chemins de fer sont tenus d'avertir dans les vingt-quatre heures la Commission dont ils dépendent de 1'apparition des sauterelles sur leurs terrains et de commencer dans le même laps de temps les opérations de lutte, à titre gratuit, à 1'aide de leur person- 
nel, de leurs outils et de leurs animaux, dont le nombre peut être augmenté si la Commission le juge nécessaire et cela aux frais des intéressés.

Un propriétaire ou un occupant dont le terrain aura été épargné par les sauterelles, pourra être tenu à collaborer avec la moitié du personnel sous sa dépendance aux travaux de lutte dans les terrains contigus.

Le propriétaire ou l'occupant devra pourvoir à l'alimentation du personnel de la propriété voisine qui lui prête sa collaboration.

Lorsque les propriétaires ou occupants n'auront pas donné avis de 1'apparition de la "langosta » et n'auront rien entrepris pour la combattre, les travaux de défense seront effectuées aux frais du propriétaire ou de 1'occupant par la Sous-commission compétente. Il en sera de même pour les entreprises de chemins de fer, lorsqu'elles négligeront de combattre les adultes de la "langosta " et de détruire les oufs et les larves dans les terrains qui leur appartiennent.

Les infractions à la loi sont passibles de plusieurs amendes, comprisestoutes entre un minimum de 5 et un maximum de rooo "pesos" (II-2200 fr). Ceux qui disposent de sauterelles ou même d'œufs détruits dans les services gratuits, pour les vendre au fisc ou pour exempter les particuliers de la présentation de ces sauterelles ou de ces œufs, sont passibles d'une amende de 50 à Ioo "pesos ) (IIo à $220 \mathrm{fr}$ ), de l'obligation de prêter leurs services gratuitement pendant une période de 5 à ro jours et de l'emprisonnement pendant le double de ce temps-là; s'il y a récidive, ces peines peuvent encore être doublées.

L'exécution de la loi est entre les mains d'une Commission centrale de "Defensa Agrícola ", composée de six membres et présidée par un Directeur général. Cette Commission pourvoit, à son tour, à l'application des prescriptions légales à l'aide d'autres Commissions, de délégués, de Commissaires ou de Sous-commissaires, détachés dans les zones les mieux appropriées, et placés sous la dépendance directe de la Direction générale de "Defensa Agrícola". Cette dernière publie même des brochures illustrées contenant des instructions pour la lutte.

Le P a r a g u a y, jusqu'à présent, n’a organisé la lutte contre leș sauterelles que dans les années où des invasions se sont produites.

Dans 1'U r ug u a y, les dispositions traitant de la défense contre les sauterelles sont contenues principalement dans la 1oi du 27 octobre Igo8 et dans la loi de "Defensa Agrícola » du 2I octobre IgII.

Obligation est faite à tout propriétaire, fermier ou occupant, de même qu'aux entreprises de chemins de fer d'aviser l'autorité compétente de l'apparition de la "langosta " sur les terrains qu'ils occupent dans un délai de 48 heures, et de pourvoir à sa destruction.

Lorsque le personnel ou les outils dont on se sert se trouvent insuffisants, les propriétaires, fermiers ou occupants sont tenus d'en informer 1'autorité compétente ; ils doivent aussi lui faire connaître l'étendue de 1'invasion, afin qu'elle puisse donner les ordres nécessaires à ses équipes, qui, avec leur matériel, coopéreront aux travaux de destruction. Dans les cas d'indigence notoire et justifiée, il est du ressort de l'autorité de pourvoir aux frais en utilisant les fonds disponibles. 
Les dispositions en vigueur pour les terrains habités sont applicables aux terrains inhabités, à part l'avis de l'apparition de la "langosta ", dont il a été fait mention ci-dessus.

En cas de négligence dans l'œuvre de destruction des insectes, d'omission de l'avis d'apparition du fléan prescrit par la loi, d'insuffisance des moyens employés dans la lutte sans que les autorités en aient été averties, celles-ci pourront, après notification, exécuter les travaux nécessaires aux frais dı propriétaire, du fermier ou de l'occupant du terrain infesté sous leur direction, s'ils le désirent, et sans préjudice des responsabilités légales dont les contrevenants seraient passibles.

Les autorités ont la faculté de pénétrer dans les terrains envahis avec le personnel nécessaire pour se livrer à la destruction des sauterelles et surveiller l'accomplissement des obligations qui incombent aux propriétaires, fermiers ou occupants de ces terrains.

Dans les terres domaniales ou communales, les établissements publics, les routes ou voies publiques, l'exécution des travaux et les frais qu'ils exigent sont à la charge des autorités respectives.

Les infractions aux dispositions en vigueur sont punies d'une amende de 30 à I0o "pesos") (I60,80 à $536 \mathrm{fr}$ ) pour les fractions de terrain inférieures à 50 ha; la même peine est appliquée pour les fractions plus grandes, plus 20 "centésimos " ( I,07 fr) par ha à calculer sur l'étendue du terrain que possède le contrevenant dans la zone visée par l'amende, sans que, toutefois, cette amende puisse dépasser, en aucun cas, la somme de I000 " pesos" (5360 fr).

Si l'amende n'est pas payée dans les dix jours qui suivent celui de l'intimation, le contrevenant subira un emprisonnement dont la durée sera calculée d'après le montant de l'amende, conformément au code pénal.

La chasse et la vente des petits oiseaux non nuisibles à l'agriculture est interdite toute l'année, sous peine de la perte du droit de chasse et d'une amende de 20 " pesos " (I07,20 fr). La même peine est applicable à ceux qui détruisent les œufs et les nids.

La Commission centrale de "Defensa Agrícola ", instituée par la loi du 2I octobre IgI I et composée de neuf membres et d'un Directeur général, qui en est le chef technique, est chargée de diriger la campagne contre la "langosta \%. Elle exerce ses attributions an moyen d'inspecteurs de zone, de section et au moyen de Commissions de section.

Le Bureau de la "Defensa Agrícola " publie des brochures illustrées sur la "langosta " et les moyens de la combattre.

Enfin la résolution du I4 aô̂t 19I5 indique les mesures de précaution à prendre dans le cas où le territoire national serait envalii par la "langosta \%.

I1 n'existe pas de dispositions législatives et administratives contre les sauterelles dans les îles Hawaï, dans l'Australie méridionale, dans la Nouve1le-Galles du Sud et dans l'Etat de Victoria. 


\section{CONVEN'TONS IN'IERNATIONATES}

Entre les diverses dispositions de cette nature actuellement en vigueur en matière de Phytopathologie, il en est nne qui se rattache tout particulièrement à la question des sauterelles.

Sur l'initiative du Gouvernement de 1'Uruguay, la Conférence internationale de "Defensa Agrícola " se réunit en mai Igr3 à Montevideo. Des diplomates et des techniciens y représentaient les pays sud-américains suivants: la Colombie, l’Equateur, le Brésil, le Pérou, la Bolivie, le Chili, le Paraguay, 1'Atgentine et l'Uruguay.

Or une Convention, signée le Io mai, et concernant précisément le Schistocerca paranensis, fut conclue, à cette occasion, entre le Brési1, la Bolivie, le Paraguay, l'Argentine et l'Uruguay.

Cette Convention comprend I 6 articles dans lesquels on a prévu la réunion, avant 1e I5 août, à Asunción del Paraguay, d'une Commission internationale, formée par un représentant pour chaque Eitat intéressé, chargée d'explorer les lieux considérés comme les foyers probables de production permanente de la sauterelle sus-mentionnée - c'est-à-dire sa zone de concentration et d'irradiation - et de proposer au besoin, à la suite de cette enquête préalable, la création d'une Station internationale centrale et de Sous-stations ayant pour but:

a) de déterminer géographiquement, d'une manière définitive, les aires de concentration de la sauterelle;

b) d'élaborer et de présenter un projet de lutte applicable à la zone de concentration, et, après son approbation, de diriger les travaux nécessaites ;

c) d'aviser les pays intéressés des mouvements des bandes, afin qu'ils puissent prendre contre elles les mesures convenables ;

d) de rédiger et de remettre, à courts interva1les, aux Gouvernements respectifs un rapport sur les travaux effectués et sur tout ce qui peut être fait pour la destruction de la sauterelle.

On a fixé, en ontre, les modalités concernant le concours financier et la collaboration réciproque entre les divers Etats contractants, pour ce qui a trait au fonctionnement de la Commission internationale, à la fondation, au fonctionnement et à la durée des Stations ; à l'application du plan commun de lutte; à l'organisation dı Service télégraphique d'avertissement de la marche des bandes de la sauterelle, en adoptant à cet effet le Codex télégraphique annexé à la Convention; à l'échange d’informations, à la fin de chaque campagne annuelle, sur le mouvement général des invasions de la sauterelle ainsi que sur les différents moyens de lutte adoptés et sur les résultats obtenus; à l'exécution, de la part des pays contractants, avec leurs propres Services et ressources, des travaux de destruction de la sauterelle dans les zones strb-permanente et temporaire placées dans leurs territoires respectifs. 
D'après ce qu'on sait, jusqu'au mois de mars IgI6, cette Convention n'avait été ratifiée que par 1'Uruguay et le Brésil.

Une seconde Conférence internationale de "Defensa Agrícola » se réunira à Buenos-Aires en IgI6.

Comme il existe une certaine connexion ou, si on le préfère, une certaine analogie entre le sujet que nous traitons et les accords intervenus entre divers pays de 1'Afrique, il convient de rappeler ici que les Eitats qui adhéraient déjà au "South African Central Locust Bureau» se sont entendus pour continuer, par l'intermédiaire de la Division d'Entomologie de Prétoria, l'œuvre de l'ancien Bureau en ce qui concerne l'engagement qu'ils avaient pris de signaler les apparitions de sauterelles et, implicitement, de détruire ces insectes.

\section{MOYENS FINANCIERS}

Il n'existe en A1le ma g ne aucune disposition à cet égard.

En P o tuga1, les frais occasionnés par les opérations de la lutte contre les sauterelles ont été, jusqu'à présent, supportés par 1'Etat. En I8gg, par exemple, les frais de la campagne contre ces insectes s'élevèrent en tout à I3 300 " escudos." (74 480 fr); l'Etat paya 20, 30 et 40 " reis ) (0, IO; 0,I5; $0,20 \mathrm{fr}$ ) le $\mathrm{kg}$ de sauterelles capturées, selon le stade de l'insecte et l'intensité de l'invasion sur les divers points du territoire infesté. Ėn Igor, la lutte coûta à 1'Etat une somme de 20000 "escudos " (II2 $000 \mathrm{fr}$ ).

Les moyens financiers employés dans la lutte contre les sauterelles en E s p a g $n$ e ont été autorisés et fixés par la loi du 2 I mai Igo8; de plus, une certaine somme est inscrite au budget du "Ministerio de Fomento" afin de couvrir tous les frais nécessaires pour combattre les fléaur des cultures; il n'est pas spécifié qu'elle doive être exclusivement affectée, comme cela a lieu en réalité, à la lutte contre les sauterelles. Un crédit de 50000 "pesetas" (50000 fr), destiné au but mentionné ci-dessus, figurait au budget de I9I4.

Lorsque 1'invasion prend des proportions alarmantes et que les provinces ne disposent pas de fonds suffisants pour la lutte, on demande an "Ministerio de Hacienda " un crédit extraordinaire approprié.

En Italie, la loi du I5 juin IgII, no 529. a autorisé, pour l'année financière IgIo-II, l'inscription d'une somme de 250000 lires au budget du Ministère de 1'Agriculture, de 1'Industrie et du Commerce, pour aider à la destruction des sauterelles, avec la faculté de conserver comme engagé pour la même destination l'excédent disponible au 30 juin IgI I. Ėin outre, le Ministère a été autorisé à émettre des mandats pour l'avance de sommes supérieures à 30000 lires en faveur des Préfets des provinces envahies, à inscrire en compte spécial; le Ministère a été également antorisé, dans des cas spéciaux et très urgents, à émettre des mandats pour le paiement d'avances, même si l'on n'avait pas encore rendu compte de l'emploi des fonds précédemment avancés. 
La loi précitée a encore établi que la moitié des frais nécessaires pour les travaux de destruction seraient supportés par l'Etat et que l'autre moitié serait répartie entre la province et les communes infestées ou menacées.

La répartition des frais est faite par le Commissaire temporaire nommé par le Ministère de 1'Agriculture, de 1'Industrie et du Commerce, sauf recours à ce Ministère.

Les communes ont la faculté de contribuer aux travaux de défense et de destruction à l'aide de prestations en nature, dont le montant est défalqué de la contribution qui leur incombe; les communes sont autorisées à imposer aux citoyens aptes au travail les prestations nécessaires; les indigents sont rétribués. Si, malgré la nécessité des travat1x, la commune ne les impose pas, le Préfet y pourvoira par une ordonnance émise sur la proposition du Commissaire.

Par le décret-loi du Lieutenant-Général du Roi d'Italie, du 27 février IgI6, $\mathrm{n}^{0} 227$, il a été inscrit au budget provisoire des dépenses du Ministère de 1'Agriculture, de 1'Industrie et du Commerce, année financière I9I5-16, la somme de 100000 lires an chapitre $n^{0} 232$ "Dépenses pour la destruction. des sauterelles $\%$.

En Autriche, l'Etat accorde, au fur et à mesure des besoins, des subventions contre les fléaux de l'agriculture; ces sommes sont complétées par une contribution appropriée que versent les Administrations locales.

On en trouve un exemple dans la campagne entreprise sur le Carso contre les sauterelles pendant l'été de Igo9; on eut recours alors à la mobilisation des élèves fréquentant les écoles de la zone envahie ; des enfants des deux sexes, âgés de Io ans et au-dessus, prirent part à la lutte et touchèrent une petite prime en argent par litre de sautereiles capturées; le litre était censé coutenir approximativement 400 insectes et était payé $0,20 \mathrm{fr}$ pendant la première semaine de lutte et $0, I_{5} \mathrm{fr}$ les semaines suivantes.

En Hongrie, en application de 1'art. 4 de la loi XXXI de I907, le Ministre de l'Agriculture avance les fonds nécessaires pour soutenir la lutte; 1'art. 5 de cette même loi dispose qu'en principe la moitié de ces fonds doit être remboursée, dans l'année, par les autorités municipales sur le territoire desquelles les travaux de destruction ont été exécutés ou, dans des cas particuliers, en plusieurs annuités; le Ministre de 1'Agriculture, d'accord avec le Ministre des Finances, peut mettre exceptionnellement à la charge de son propre budget jusqu'aux ${ }^{2} / 3$ des frais nécessités par les travaux de lutte contre les sauterelles. Les émoluments du personnel d'experts délégués par 1'Etat pour conduire les travaux de lutte, ainsi que le coût des appareils spéciaux, sont entièrement supportés par 1'Etat. Ėn échange, les municipalités doivent fournir et surveiller les attelages, les bêtes de trait et la main-d'œuvre nécessaires.

La Roumanie a des crédits spécialement affectés à la lutte contre les sauterelles.

Le tableau suivant indique quelques-uns des crédits supérieurs à $5000 \mathrm{fr}$ qui furent affectés à cette lutte en diverses années : 


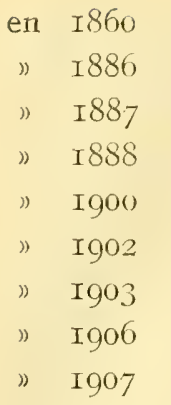

$280000 . \mathrm{fr}$
$15000 "$.
$30000 "$
$20000 \quad "$
54245
6000
10000
23225
18000

Dans le budget du Ministère de l'Agriculture et des Domaines de la Bulgarie, on prévoit une somme globale pour toute lutte contre les maladies et les ennemis des cultures. En IgI2, cette somme s'éleva à $5000 \mathrm{fr}$.

De plus, quelques Commissions départementales permanentes affectent chaque année des sommes de 500 à Iooo fr à la lutte contre les fléaux de 1'agriculture dans leurs départements respectifs.

Ein IgII et en I9I2, un crédit de I50 000 fr fut inscrit, en Grèce, au budget de 1'Eitat pour la destruction des sauterelles. Il fut établi que quiconque découvrirait ou indiquerait un lieu de ponte toucherait une prime à prélever sur ce crédit. On alloua une prime de I fr par ocque ( I $280 \mathrm{~g}$ ) d'œufs ramassés; quatre ocques environ n'en représentaient plus qu'une, lorsque les œufs étaient débarrassés de la terre qui les enveloppait. Chaque ocque de sauterelles était payée de 0 , Io à 0,30 fr selon 1'âge et par conséquent suivant la grandeur de ces insectes.

En C h i n e, les crédits nécessaires pour combattre les sauterelles sont fournis par le Gouvernement, mais il n'y a pas de contribution fixe, permanente à cet effet. Le crédit est accordé au fur et à mesure des besoins.

Danıs 1'I n d e, des sommes spéciales sont allouées à la lutte lorsque les circonstances l'exigent.

Lorsque la population ne participe pas spontanément aux travaux de destruction, on établit quelquefois, afin de l'encourager, des primes dont le montant est en proportion des œufs ramassés ou des adultes capturés soit pendant leur vol, soit lorsque, engourdis par le froid, ils restent juchés sur les branches des arbres.

Ainsi, en I904, le Gouvernement de Bombay dépensa en tout pour ces primes un peut plus de 200000 roupies (336000 fr); durant cette campagne dirigée contre le Cyrtacanthacris succincta, on arriva à détruire 66432000 adultes, 400000000 d'œufs et 530000000 d'insectes $110 n$ ailés.

Ia loi XII, promulguée à Chypre le Io août I88I, établit les règles pour établir les impôts dont le produit sert à convrir les frais nécessaires pout la destruction des sauterelles dans l'île.

Le Gouvernement de la Tun isie vote des crédits prélerés sur les disponibilités budgétaires et mis à la disposition de la Direction générale 
de 1'Agriculture, qui a la charge de l'organisation de la lutte contre les sauterelles. Ces crédits ne sont pas limités et sont délégués lorsqu'il en est besoin. Dans certaines circonstances, 1'Administration distribue aux indigènes une prime fixe par chaque "guelba " (20 litres) d'œufs de sauterelles ramassés.

Le décret du 27 décembre I9I 5 autorise le prélèvement sur les fonds des excédents disponibles d'un crédit en vue de la défense contre les sauterelles.

En Erythrée, le Gouvernement pourvoit aux moyens financiers nécessaires pour combattre les sauterelles au fur et à mesure des besoins.

Dans la Province de Mozambique, le district de Quélimane, qui comprend le Bas-Zambèze, dispose d'un fonds permanent pour parer aux cas urgents.

I e Gouvernement du Sud-Ouest africain allemand a annuellement à sa disposition une somme de Io 000 marks (I2 $346 \mathrm{fr}$ ) pour la campagne contre les sauterelles.

Pour ce qui concerne 1'Union de 1'Afrique du Sud, le budget de la Division d'Entomologie du Département de 1'Agriculture comprenait, pour l'exercice I9I3-I4, une somme de 250 livres sterling (6 $305 \mathrm{fr}$ environ) destinée à 1a 1utte contre ces insectes.

Aux Etats-Unis d'Amérique il n'y a pas de crédit spécial affecté à la lutte contre les sauterelles: les sommes destinées à combattre les insectes en général sont établies par le Congrès; elles sont inscrites au budget général de 1'agriculture.

A Costa-Rica, par décret du 20 mai IgI5 le pouvoir exécutif a été autorisé à dépenser les sommes nécessaires pour la lutte.

Ise Gouvernement a décidé de payer I "colon " $(2,40 \mathrm{fr})$ par Ioo livres (46 kg) de sauterelles capturées; Ioo livres comprennent environ 25000 insectes. Cette méthode a donné d'excellents résultats.

Dans l'ile de Saint-Christophe (St.-Kitts) on a fait procéder quelquefois au ramassage à la main des insectes contre une petite prime en numéraire par centaine de sauterelles capturées.

Le Conseil de 1'Agriculture de la Trinité a la faculté de pourvoir immédiatement aux moyens nécessaires pour combattre toutes les apparitions importantes des sauterelles.

Pendant l'invasion des sauterelles qui eut lieu dans le comté de Berbice en I886, le Gouvernement de la Guyane anglaise offrit, pour la destruction de ces insectes, les primes suivantes payées sur les recettes de la Colonie: 2 schellings (2,50 fr environ) par "gallon" (4,54 litres environ) de jeunes insectes et d'œufs présentés séparément ou ensemble et 6 "pence " $(0,60 \mathrm{fr}$ environ) par "gallon " d'adultes.

Quelques personnes, principalement des planteurs, furent autorisées par le Gouvernement à avancer le payement de ces primes d'après le tarif indiqué. 
Au Pérou, dans quelques cas où les sauterelles se sont présentées avec un certain caractère alarmant, le sous-préfet de la circonscription menacée a reçu une petite somme qui, généralement, a été employée d'accord avec les propriétaires pour payer le ramassage des insectes ailés.

Une somme de 5000 "libras peruanas" (I26 I07 fr) a été inscrite au budget général de I9I5 pour combattre systématiquement le fléau.

Dans 1'Argentine, à partir de IgII le Gouvernement fit évaluer le montant probable des frais que pouvait exiger la lutte contre la "langosta » et les fonds nécessaires à cet effet ont été inscrits à la loi de finances.

Les frais relatifs à 1'exécution des travaux ont été réduits peu à peu : I) grâce à l'acquisition d'une plus grande quantité de barrages en zinc, qui, comme on le verra, coustituent le moyen de lutte le plus efficace employé dans le pays; cette acquisition a facilité notablement la destruction, en permettant de réduire de beaucoup le personnel destiné à la lutte ; 2) en supprimant 1'achat des insectes et des œufs ramassés fait précédemment par 1'Etat; 3) en supprimant les équipes auxiliaires qu'on n'utilisait plus que dans des circonstances exceptionnelles.

Actuellement enfin, on ne procède plus à la distribution gratuite, pas même contre remboursement, des produits inflammables et des liquides destinés à la destruction des sauterelles; le matériel nécessaire est 1oué au public moyennant une somme de 3 "centavos" (0,07 fr environ) par mètre courant de barrage en zinc pour toute la durée des opérations. Le prix de location est certainement insignifiant, car il suffit à peine à couvrir les frais de transport et de réparation des barrages; toutefois, il constitue une économie pour le Trésor public; quant aux agriculteurs, ils ne confient plus ainsi exclusivement à l'action officielle la défense de leurs propres intérêts.

Voici quel a été le montant des sommes employées annuellement à la lutte contre le fléau de Igo6 à I9I4:

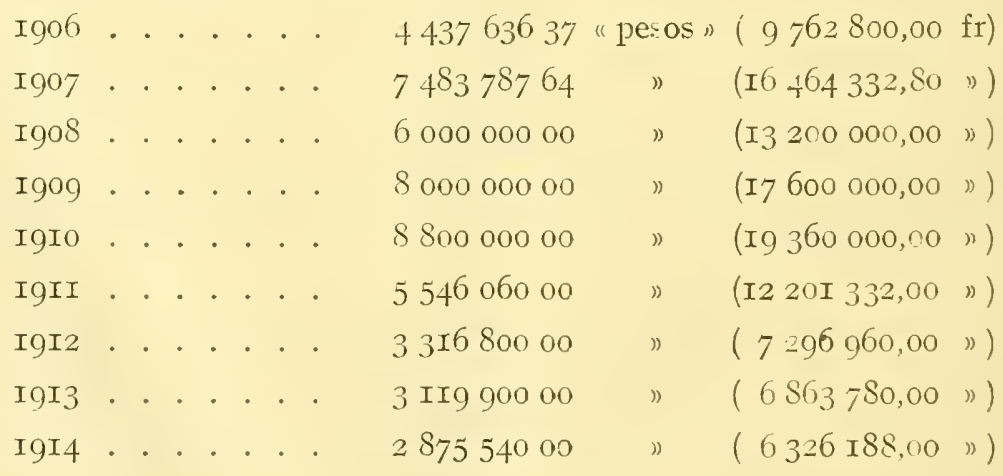

Au Paraguay, les sommes destinées à la lutte contre les sauterelles ont été proportionnées jusqu'à présent à 1'importance et à la durée des invasions. 
Le Gouvernement de 1'Uruguay, dans les années d'invasion, détermine la somme nécessaire pour couvrir les frais occasionnés par les opérations de lutte. Cette somme varie selon la gravité de l'invasion. En I9r4, par exemple, elle s'éleva à 50000 "pesos » (255000 fr).

Aucun crédit spécial affecté à la lutte contre les sauterelles n’existe actuellement dans les îles $\mathrm{Hawa}$, dans l'Australie méridionale, dans la Nouvelle-Galles du Sud et dans l'Etat de Victoria.

\section{MOYENS ET INITIATIVES PRIVÉS}

Au Portuga1, les agriculteurs des régions envahies par les sauterelles contribuent de leurs propres deniers aux opérations de lutte et de destruction.

En Espagne, quelques Administrations particulières prennent des mesures déterminées pour contribuer au succès de la campagne contre le fléau, mais elles n'en ont presque jamais l'initiative et se servent des indications des "Consejos de Fomento » de leurs provinces.

Quelques corporations prennent sur leurs propres fonds pour acheter le matériel qu'il faut pour la lutte et pour payer la main-d'œuvre journalière, sans avoir recours aux fonds du "Ministerio de Fomento " ni à ceux que la 1oi du 2I mai Igo8 autorise à percevoir des provinces.

En G r è c e, parallèlement à l'action du Gouvernement, les Sociétés agricoles, les Unions et les Syndicats des cultivateurs, les grands propriétaires, etc. ont contribué, en IgII-I2, dans une large mesure, moralement et pécuniairement, à l'œuvre entreprise par 1'Etat pour combattre les sauterelles.

La Société royale d'Agriculture hellénique, en particulier, après avoir décidé que des agronomes concourraient aux opérations de défense, a publié un bulletin spécial qui relate les meilleurs moyens de destruction; elle a également entrepris à partir de IgII, avec la collaboration des instituteurs, de dresser une carte annuelle du pays, où l'on indiquerait les parties du territoire dévastées l'année précédente et l'étendue des dommages constatés. Comme on n'a pas organisé jusqu'ici de Service de recherches à cet effet, cette carte n'indique pas, ce qui serait fort utile, les lieux de ponte, mais elle rend quand même des services pratiques en permettant de connaître avec une grande approximation au moins les points où l'on doit s'attendre à l'éclosion des sauterelles et, par conséquent, de prendre en temps voulu toutes les mesures nécessaires pour combattre ces insectes dès leur naissance.

Il n'existe en Chine ni Sociétés ni Syndicats qui concourent à la lutte contre les sauterelles, mais 1'initiative privée s'occupe de la propagande des moyens de défense contre ce fléau.

Dans les localités de l'Inde souvent visitées par ces insectes, la 
population, en général, comprend fort bien la nécessité de combattre le fléau sans retard; elle vient donc volontiers et en bon nombre à l'aide des fonctionnaires du Gouvernement. L,e plus souvent, l'efficacité de la campagne réside précisément dans la coopération spontanée des habitants des zones envahies.

En 'Tunisie, la direction de la lutte a été laissée jusqu'ici à 1'Administration, mais les principaux propriétaires et les Associations de colons ont prêté efficacement leur aide dans leurs circonscriptions respectives.

Dans l'Afrique orientale allemande, les particuliers participent au Service d'avertissement contre les sauterelles en signalant leur apparition au moyen de cartes postales; et en général, dans les Colonies sud-africaines, les agriculteurs et les indigènes coopèrent en outre le plus souvent avec leurs Gouvernements respectifs aux diverses opérations de destruction.

Au Mexique, de nombreux propriétaires se chargent à leurs frais de la lutte contre les sauterelles.

Avant que le Gouvernement de la Guyane anglaise n'établît des primes spéciales pour la destruction des sauterelles lors de l'invasion qui frappa le comté de Berbice en I886, quelques propriétaires de plantations de canne à sucre entreprirent la lutte à leurs frais chacun sur son propre terrain.

Dans l’Argentine, les Sociétés et les Syndicats intéressés à la défense des cultures contre les ravages de la "langosta " ne s'associent que fort peu à l'action officielle. Les bourses de céréales, les marchés de céréales et les Sociétés rurales de l'intérieur ont servi néanmoins de trait-d'union entre les agriculteurs et les autorités chargées di contrôle et de l'application de la loi $n^{\circ} 3708$; ils ont transmis les plaintes que cause inévitablement une action rapide et énergique comme celle de la lutte contre les sauterelles et ont fait connaître les besoins des régions envahies.

On travaille depuis deux ans à développer, dans ces Associations, 1'intérêt que la question mérite et, par la nomination de délégués ad honorem de l'agriculture et de la défense agricole, on a formé des corps auxiliaires très précieux en ce sens qu'ils resserrent toujours davantage l'union nécessaire entre les agriculteurs et les autorités; de plus, ils mettent leur influence au service de l'intérêt général dans le but d'obtenir de tous la plus grande activité possible dans l'exécution des travaux de destruction.

Les entreprises de chemins de fer, constituées, pour la plis grande partie, avec des capitaux étrangers, apportent, à leur tour, une collaboration digne de considération en ce qu'elles avisent la Division de "Defensa Agrícola " des mouvements des bandes envahissantes, observées dans la zone d'influence de leurs concessions respectives, ce qui évite d'envoyer par anticipation un personnel rétribué sur les points stratégiques on apparaissent d'ordinaire les premières bandes.

La collaboration pécuniaire apportée par les Associations et les Syndicats agricoles pour la lutte contre les sauterelles est absolument in1le; on 
n'a pu obtenir que celle des voisins et du commerce local des régions envahies, et cela grâce à l'insistance du personnel de la "Defensa Agrícola ». Dans les cas où les propriétés de la population urbaine étaient menacées, ce personnel institua des Commissions qui, à l'aide de souscriptions volontaires, réunirent des fonds communs pour organiser des équipes destinées à la lutte.

I' "Asociación rural del Uruguay", dont le siège est à Montévidéo, se livre, à l'aide de sa "Revista ", de feuilles volantes et d'autres moyens, à une propagande active parmi les agriculteurs en faveur d'une lutte vigoureuse et rationnelle contre le fléau qui désole la République de l'Uruguay. 



\section{MOYENS DE L U'T'TE LN USAGE DANS LES DIVERS PAYS}

\section{MOYENSS NA'TURELS}

A tous les stades de leur évolution et, pratiquement, dans toutes les régions du globe, les sauterelles sont en butte aux attaques d'ennemis des plus variés qui, dans la nature, peuvent en réduire considérablement le nombre et la propagation.

La série de ces auxiliaires naturels de 1'agriculture dans sa lutte contre ces redoutables orthoptères est déjà longue, malgré qu'elle soit encore incomplète, puisque même récemment on a signalé ou décrit quelques nouveaux ennemis des sauterelles, et il n'est pas improbable qu'on en découvrira d'autres encore dans l'avenir. Actuellement, on en compte dans le règne végétal, parmi les bactéries et les champignons, et encore plus dans le règne animal parmi les vers, parmi les arthropodes (arachnides, myriapodes, insectes) et parmi les vertébrés (amphibies, reptiles, oiseaux et mammifères).

Il était naturel que, surtout à cause de l'abondance de quelques-uns des champignons connus comme parasites des sauterelles et de l'efficacité dont ils ont fait preuve spontanément dans des circonstances de milieu déterminées, on pensât à la possibilité de les cultiver artificiellement sur une vaste échelle pour les employer, au moment voulu, contre les orthoptères ravageurs.

Des essais nombreux et plusieurs fois répétés ont été faits dans toutes les parties du monde, dans les conditions de milieu les plus variées et d'après la technique la plus rigoureuse; mais jusqu'ici les espérances que l'on avait conçues à cet égard se sont heurtées le plus souvent contre des difficultés pratiques insurmontables. Si les essais d'infection artificielle ont parfois donné des résultats positifs au laboratoire, ils ont eu, par contre, du moins jusqu'à présent, des effets fort indécis, pour ne pas dire qu'ils ont complètement échoué, lorsqu'il s'est agi de les appliquer en plein champ sur des sauterelles vivant en liberté.

Le seul représentant du règne végétal, qui — tout en étant d'ailleurs encore à la période expérimentale - si l'on en juge surtout par de très récents résultats obtenus dans les Colonies françaises de 1'Afrique du Nord, semble réanmoins s'annoncer comme un moyen complémentaire utile pour la lutte c'est le Coccobacillus Acridiorum d'Hérelle, découvert au Mexique, dans 1'Etat de Yucatan. 
Depuis longtemps, on a pratiquement adopté dans divers pays, comme moyen facile et assurant un résultat rapide, l'emploi de quelques animaux domestiques bien connus pour se nourrir assidûment de ces orthoptères et de leurs œufs.

Ainsi, en Espagne on a coutume de conduire des trcupeaux de porcs dans les zones occupées par les coques ovigères dont ces animaux son très friands; on force les gardiens des troupeanx et les gardes champêtres à ne pas abandonner une localité infestée avant que le terrain n’ait été soigneusement fouillé. Cette manière d'assainir le sol est généralement employée là où l'on ne peut utiliser ni la charrue ni l'extirpateur à cause des roches ou des buissons épais qui entourent parfois les espaces de peu d'étendue.

On transporte aussi sur les points envahis par les sauterelles des troupeaux de dindons et de poules; on se sert quelquefois pour cela de poulaillers portatifs; la volaille consomme une quantité importante d'insectes, pourvu que 1'eau ne manque pas.

En Italie, et plus spécialement dans la province de Cagliari (Sardaigne), on a souvent recours aux animaux pour détruire les sauterelles. Même dès les temps les plus reculés, on conseillait, lorsqu'on n'en donnait pas 1'ordre, de faire paître des pores dans les terrains remplis d'œufs de sauterelles. D'après des observations faites sur place, les porcs découvrent de loin la présence des lieux de ponte vers lesquels ils se précipitent pour se mettre incontinent à creuser fébrilement la terre avec leur groin et se repaître des œufs ; leur voracité est telle qu'elle provoque de véritables batailles entre les animaux d'un même troupeau. Ces œufs constituent une excellente nourriture pour le porc qu'elle fait engraisser sans qu'il ressente aucun trouble; sa chair en acquiert, paraît-i1, une saveur assez désagréable, mais cet inconvénient disparaît complètement en mettant l'animal à un autre régime un mois avant de l'abattre.

I1 en résulte, d'après ce que l'on a pu constater à plusieurs reprises, que le porc est un excellent auxiliaire pour rechercher et détruire les lieux de ponte ; c'est la raison pour laquelle on a même exprimé le désir qu'une mesure législative oblige à l'avenir les propriétaires des terrains incultes, occupés par des œufs de sauterelles, d'en permettıe d'octobra en février 1'accès et le pâturage à des porcs appartenant à d'autres éleveurs, lorsqu'ils n'y pourvoient pas eux-mêmes avec leur propre troupeau.

En Autriche, les dindons ont été introduits et propagés sur le Carso gorizien, depuis I907, avec d'excellents résultats, ce qui permettait d'affirmer déjà en Igog que, si l'on avait pu disposer d'un nombre suffisant de ces volatiles, on aurait obtenu de ce fait une diminution considérable de santerelles.

On a reconnu en Grèce aussi que, parmi les oiseaux de basse-cour, les dindons surtout étant très friands de sauterelles, sont d'une grande utilité dans la lutte contre ces orthoptères, et que, s'il se trouve des troupeaux de dindons en assez grand nombre dans une localité donnée, on peut être assuré qu'ils dévoreront tous les insectes présents et préserveront la contrée des dégâts qu'ils auraient occasionnés. Il paraît que cet 
aliment engraisse merveilleusement les dindons; c'est ce que l'on a constaté dans le district de Mégalopolis très riche en dindons et qui, de ce fait, est rarenent ravagé par les sauterelles. Il est vrai que 1'on reproche à la chair des dindons ainsi nourris de conserver une odeur très caractéristique. In supprimant cette nourriture I5 ou1 20 jours avant de tuer la bête, toute odeur de ce genre aura disparu.

C'est précisément à cause de leur utilité pratique que le Gouvernement grec conseille beaucoup la multiplication des dindons, afin qu'on puisse les employer largement daus la destruction des sauterelles.

Parmi les moyens de lutte adoptés au Japon figure l'emploi des poules ou des dindons.

En Chine, lorsque les sauterelles apparaissent et s'abattent sur les rizières, on y lâche immédiatement des canar̂ds, ce qui donne de bons résuitats pratiques.

En 'Tunisie, au cours d'une des dernières campagnes de lutte, un agriculteur s'est servi des porcs avec grand succès pour combattre une bande de sauterelles. A la tombée de la nuit, alors qu'il était certain que les insectes ne reprendraient pas leur vol, il amena sur 1'emplacement qu'ils occupaient un troupeau de porcs qui dévora en fort peu de temps la plus grande partie des ailés qui composaient la bande.

On n'ignore pas aux Etats-Unis d'Amérique l'utilité de l'emploi des dindons et des poulets pour la destruction des sauterelles, et cette utilité est confirmée, à chaque nouvelle invasion, dans les régions où elles constituent un fléau annuel; toutefois ce moyen de lutte n'est pas encore très largement répandu.

Dans les cinq ou six dernières années du XIX'me siècle, dit M. F. B. MILLIKEN (I9I5), un grand nombre de dindons furent élevés dans ce but dans les terrains situés le long du fleuve Solomon, dans le Kansas septentrional. La méthode fut reconnue tellement efficace, que les agriculteurs. de la région en étendirent de plus en plus chaque année 1'application jusqu'à la fin de la série des invasions.

En I9II, toujours au Kansas, non loin de Scott City, la seconde récolte de luzerne était gravement endommagée dans un champ de 40 ha. I, propriétaire eut 1'idée d'y transporter et d'y laisser circuler librement une centaine de dindons. Lorsque M. MILIIKEN s'en alla visiter la propriété dans la troisième semaine d'août, il ne restait plus dans le champ que quelques exemplaires de Melanoplus atlanis qui avaient échappé en volant au bec avide des dindons. La luzerne n'avait plus été touchée, elle avait même atteint une hauteur d'environ $50 \mathrm{~cm}$.

La "Kansas Agricultural Experiment Station " de Garden City fit l'acquisition de plusieurs centaines de poulets pour essayer de sauver ses cultures pendant la grande invasion de sautereiles de I9I3. On fit usage des poulaillers portatifs pour se rendre d'un lieu infesté dans un autre, ainsi que M. MIIIKEN 1'avait déjà fait avec succès 1'année précédente; 
et cette méthode fut considérée comme le moyen le plus efficace de détruire ces insectes.

M. MrLitres raconte encore qu'en I9I3, la rhubarbe d'un jardin situé près de Garden City était gravement attaquée par les sauterelles, qui en dévoraient en peu de temps les feuilles. Le jardinier plaça près des plantes et dans des mues séparées trois poules avec quarante poussins en tout. Quelques jours après, la rhubarbe était délivrée des sauterelles et les poussins allaient à la chasse d'autres exemplaires de ces insectes qui se trouvaient dans le voisinage du jardin.

Ontre la volaille, les pores de tout âge ont montré aussi aux Eitats-Unis qu'ils sont des destructeurs excellents des sauterelles et de leurs œufs. Une petite partie d'un champ que l'on avait laissée pour y récolter de la graine de luzerne fut close pour la livrer à la pâture de quelques porcs. Les sauterelles endommagèrent le champ entier, sauf la partie réservée aux porcs où l'on eut une bonne récolte de semences.

Aux petites Antilles, et plus particulièrement dans l'île de SaintChristophe (St. - Kitts), des résultats encourageants ont été obtenus, lors des apparitions de sauterelles, en faisant séjourner dans les terrains infestés des troupeaux de poules, de dindons et de pintades, qui ont en même temps donné la chasse à d'autres insectes

Dans 1'Etat de Victoria (Australie), les dindons sont considérés également comme un des moyens les plus efficaces de détruire les sauterelles; lorsqu'ils s'en nourrissent, ces volatiles, arrivés à un tiers environ de leur développement, engraissent rapidement, de sorte que non seulement ils sont utilisés pour enrayer le fléau, mais ils arrivent par cela même à le convertir en une source de profit.

Nous avons eu 1'occasion de rappeler dans un autre chapitre que, tout en étant omnivores et voraces, les sauterelles respectent quelquefois ou dédaignent, selon les pays, certaines plantes tant spontanées que cultivées.

On a tiré parti de ce fait et de l'expérience acquise sur les mœurs des sauterelles en Chine, et l'on a par suite pris dans ce pays une précaution culturale qu'il nous semble bon de signaler ici, avant de passer à d'autres sujets, puisqu'elle rentre dans la catégorie des moyens naturels de lutte.

Lorsqu'une invasion de sauterelies s'est vérifiée en Chine, on ne sème pas de graminées l'année suivante dans les champs éprouvés, cas plantes étant les seules que les sauterelles dévorent dans le pays, mais plutôt des espèces végétales qui n'attirent pas ces insectes, le soya (Glycine Soja) par exemple. Ainsi disparaissent les dernières traces des sauterelles, les insectes survivants ne trouvant plus à se nourrir.

D'autre part, dans 1'Etat de Victoria, la plantation de Delphinium spp. ou de Ricinus communis sur les bords des enclos est considérée comme un moyen de lutte très efficace, car les feuilles de ces plantes sont vénéneuses pour les santerelles. 
Pour capturer les jeunes orthoptères on se sert en Fir a nce, daus la Camargue, de pièces de toile forte, appelées "tentes» dans le pays, et d'une longueur de I2 m sur 4 de large; elles sont munies, le long de la ligne médiane, à une distance de 2,5 à 3 m des deux extrémités, d'une fente de $35 \mathrm{~cm}$ à laquelle est adapté un sac dont le foud, au lieu d'être cousu, est lié avec une ficelle.

Une moitié environ de chaque tente est étendue sur le sol, à une distance convenable des insectes, tandis que l'autre moitié est maintenue dans une position verticale par des ouvriers qui en soutiennent le bord à hauteur d'épaule; d'autres ouvriers, placés devant la tente et munis de branches, chassent peu à peu les insectes vers la partie de la toile étendue sur le sol; lorsqu'un grand nombre d'insectes s'y trouvent rassemblés, on soulève cette partie de la toile pour la rapprocher de celle qui a conservé la position verticale et on secoue le tout de manière à étourdir les orthoptères capturés; ceux-ci tombent dans le sac à travers la fente qui est pratiquée dans la tente. Lorsqua le sac est plein, on dénoue la ficelle qui en ferme le fond et les insectes sont versés dans des sacs à grains ordinaires afin de pouvoir les détruire ensuite plus facilenent.

Le procédé le plus généralement employé en Portugal est analogue au précédent et il y donne des résultats immédiats. Ce procédé consiste en effet dans la capture des insectes à l'aide de barrages mobiles formés d'une ou de plusieurs toiles ou de grandes bandes de tissu de $60 \mathrm{~cm}$ à I $\mathrm{m}$ de haut, munies à leur centre d'une fente à laquelle est adapté un sac dans lequel tombent les insectes qu'une équipe d'ouvriers pousse à l'aide de branches vers les barrages. Ce travail se fait avec plein succès le matin et le soir, sauf aux heures les plus chandes. Ce procédé a permis de capturer, en I899, $559559 \mathrm{~kg}$ d'orthoptères; il fallait en moyenne I5 ooo insectes pour former le poids d'un kilogramme. En Igor, on détruisit ainsi I I36 $896 \mathrm{~kg}$ de ces orthoptères.

On a employé aussi, dans ce pays, le brûlage des insectes adultes à 1'aide de feux et de torches; cette méthode a donné des résultats pratiques partout où 1'on put 1'appliquer sans danger pour la végétation environnante.

Pendant la campagne de I902, on a constaté que le brûlage au moyen de la flamme de gazoline est un des procédés de destruction les plus efficaces pourvu qu'on commence à 1'appliquer dès 1'éclosion des insectes.

En Espagne, on emploie plusieurs moyens mécaniques et physiques pour combattre les sauterelles dans leurs divers stades d'évolution.

En premier lieu, on a recours à des machines qui travaillent la terre très superficiellement de manière à couper les coques ovigères qu'elle contient pour provoquer ainsi la putréfaction des cufs et pour laisser ces derniers à découvert afin qu'ils deviennent la proie des oisea11x et que les intempéries puissent produire lenrs effets bienfaisants. On se sert à 
cet effet d'extirpateurs ou bien, si ces instruments font défaut, de charrues de divers systèmes propres à travailler la terre le plus superficiellement possible et qui tracent des sillons unis; comme outils complémentaires on emploie les bêches et les pioches.

On pratique aussi le ramassage des coques ovigères au moyen de petites pioches et on les sépare de la terre à l'aide de cribles.

Parfois des équipes d'ouvriers armés de branches et de grands balais donnent la chasse aux sauterelles aux premiers stades de leur évolution, à savoir lorsqu'elles sont encore à l'état de larves à peine nées ("mosquitos ") et lorsqu'elles ont déjà subi quelques mues (" moscas ") ; ils les rassemblent, les assomment et les enterrent dans des fosses où ils les écrasent à l'aide de battes.

On fait de plus un grand usage, en Ëspagne, de barrages en zinc contre les sauterelles au stade de "mosca " et de "saltón" (insecte déjà muni des moignons alaires); lorsqu'on connaît la direction prise par les insectes et la vitesse avec laquelle ils s'avancent, on creuse avec la charrue des sillons à une distance convenable l'un de l'autre et on dresse contre ceux-ci des bandes en zinc au moyen de pieux (en Esspagne on emploie à cet usage les vielles baïonnettes); on forme ainsi une grande courbe ouverte dans la direction d'où provient la bande des orthoptères. Dans l'intérieur de l'enceinte, on creuse des fosses où les insectes tombent et où on les détruit à l'aide du "zotal » dont nous parlerons plus tard, ou bien en projetant sur la masse des insectes, au moyen de pulvérisateurs de différents modèles, de la gazoline que l'on allume ensuite et qui donne une flamme d'un grand pouvoir destructeur; ou bien encore, on les écrase à l'aide de battes. La gazoline ne doit pas être mise en contact avec les bandes en zinc, car sa flamme les détériore.

Les ouvriers donnent aussi la chasse aux sauterelles (stades de " mosquito ", de "mosca " ou de "saltón ») en les réunissant en de gros tas dans un endroit clos de branches sèches auxquelles on met le feu; on pousse ensuite ces branches enflammées vers le centre de l'enclos même, au moyen de bâtons ou de fourches.

On se sert également de toiles au milieu desquelles s'ouvre un large sac. On les place sur le sol, à proximité des bandes de jeunes insectes ("mosquitos» ou "moscas») que l'on veut détruire et on oblige les insectes à entrer dans le sac; lorsqu'il est plein on le vide dans des fosses où les orthoptères sont détruits au moyen de battes ou à l'aide dı feu.

Comme nous venons de le dire, la gazoline est d'abord pulvérisée et puis enflammée sur les fosses contenant lesinsec tes capturés au moyen des barrages en zinc; toutefois le liquide ne doit pas être mis en contact avec ces derniers qui en seraient gravement détériorés.

Mais la gazoline est plus généralement employée comme combustible en plein champ. Ce procédé est facile à employer dans les premières heures du matin et du soir lorsqu'on peut surprendre les jeunes orthoptères ("mosquitos 》) rassemblés et presque immobiles. Dans la journée, lorsque l'air est plus chaud, les insectes se mettent en mouvenent; on les poursuit alors et on les rassemble en prenant bien soin qu'il n'en reste 
pas en arrière - on en fait de même avec les "moscas" - et l'ouvrier qui manœuvre le pulvérisateur - plusieurs modèles de cet appareil sont en usage - pénètre au milieu de la bande d'insectes et, marchant le dos tourné au vent, il arrose rapidement les insectes, tandis qu'un autre ouvrier y met le feu et que d'autres se chargent de pousser les insectes vers le foyer et de les y entasser.

En Italie aussi on a maintenant plusieurs manières de combattre les sauterelles.

Nous rappellerons ici que 1'on y pratique le ramassage et la destruction des coques ovigères, que l'on s'y livre à la capture des jeunes insectes au moyen de toiles et d'un appareil collecteur d'un système spécial, et que l'on y emploie aussi la flamme de pétrole mélangé à ro \% de benzine qui permet au pétrole de s'enflammer plus facilement et d'obtenir une combustion plus complète.

Pour le ramassage des coques ovigères, après avoir déterminé d'avance et avec précision la situation et l'étendue des foyers de ponte, on bêche le sol à 4 ou $5 \mathrm{~cm}$ de profondeur et on amoncelle la terre ainsi remuée, puis on la passe au crible. Les herbes et la terre qui restent dans les cribles sont de nouveau mises en tas par des femmes; celles-ci retirent les coques ovigères qui, enfermées dans des sacs, sont transportées dans des fosses à fumier spéciales. Comme, malgré le criblage, une assez grande quantité de coques ovigères (jusqu'à $6 \%$ ) restent entre les racines des herbes, on met ces dernières en tas, on les laisse sècher et on les détruit ensuite en les brûlant.

Du 26 octobre I9I3 au 30 mars I9I4, on a ramassé en Sicile--et pour être plus précis dans les communes de Bronte et de Maletto (prov. de Catane) qui en ont fourni les $2 / 3$ - sous la direction de Mr. LUNARDONI, 997 q d'œufs de sauterelles, représentant 6500 millions d'insectes; les frais de destruction s'élévèrent à 3 I $000 \mathrm{fr}$. Il vaut mieux ne pas procéder au ramassage des coques ovigères lorsqu'elles arrivent à couter plus de o,6o fr le kg.

Les toiles employées pour la capture des jeunes insectes sont blanches ou à peu près, solides, longues de $6 \mathrm{~m}$ et larges de $5 \mathrm{~m}$; comme on a déjà eu 1'occasion de le dire dans d'autres cas, elles sont munies au milieu d'une fente à laquelle a été adapté un sac. Le fonctionnement de la toile est à peu près le même que celui que nous avons décrit précédemment; seulement, au lieu d'être soutenue à moitié, dès le début del'opération, dans une position verticale, la toile est complètement étendue sur le sol et fixée à l'aide de pierres que l'on place sur les bords et aux angles. Lorsque les insectes que 1'on pousse vers la toile sont sur le point de la dépasser, on en relève une moitié dans le sens opposé à la direction suivie par les insectes de manière à former une paroi verticale. Avec une seule toile, en douze heures seulement, lorsque les orthoptères étaient en grande partie ailés, M. LUNARDONI en a pu faire capturer jusqu'à 8 quintaux, soit de 800000 à 900000 insectes. Du I6 avril au II juillet I9I4, environ $90000 \mathrm{~kg}$ d'insectes sur le point de devenir adultes, ou déjà ailés ont été capturés avec la méthode des toiles rans les deux communes de Bronte et de Maletto. 
M. LunaRdoni a imaginé en IgI3 et fait construire en IgI4 un modèle de collecteur qui, chose impossible avec les toiles, peut être utilisé les jours de vent, sur n'importe quel terrain et même lorsque ces orthoptères font peu de chemin. Il s'agit d'une armature de métal large de 3,30 m, à plan inçliné sur laquelle est tendue une toile de $3 \mathrm{~m}$ de large destinée à recueillir les insectes que des ouvriers poussent dans sa direction; quand 1a toile est couverte d'un grand nombre d'insectes, on la roule lentement sur elle-même au moyen d'une manivelle placée dans le haut sur un côté de 1'appareil; les orthoptères se trouvent ainsi entraînés vers la partie supérieure du plan incliné d'où ils tombent dans deux trémies en zinc, qui se vident par en bas dans un sac. Ce collectenr permet de capturer presque tous les insectes à condition de faire repasser les équipes d'ouvriers sur le terrain déjà battu. De plus si 1'on fait accomplir à l'appareil un tour de $90^{\circ}$ chaque fois, on peut, en restant à la même place, débarrasser des insectes une bonne étendue du terrain. Ce collecteur, démontable et facile à transporter, a été depuis perfectionné.

Pour appliquer la méthode de la flamme de pétrole mélangé de benzine, on emploie des pulvérisateurs. On se sert à cet effet des pulvérisateus à air comprimé "Pomonax» et "Calimax» de fabrication allemande, comme on se sert également de pulvérisateurs à pédale et à seau construits en Italie, sauf à leur faire subir les quelques modifications ou perfectionnements dus à l'expérience acquise par M. LUNARDonI pendant la campagne qu'il dirigea en Sicile de IgI2 à IgI4.

Ainsi, dans les pulvérisateurs de fabrication allemande, on a appliqué, au point de jonction du tube de sortie du liquide sous pression, un interrupteur, soit pour avoir la facilité d'arrêter soudainement le courant du liquide ou encore de nettoyer le jet s'il venait à se boucher, soit enfin pour éviter que la pression du liquide sur les tubes de caoutchouc ne les use trop vite et ne les fasse éclater. La modification apportée à la construction des pulvérisateurs de fabrication italienne consiste en ce que les lances sont en cuivre jaune et qu'elles ont de 2,5 à $3 \mathrm{~m}$ de 1 ongueur, afin d'éviter le danger de la flamme que le vent ramène facilement en arrière; et, pour supprimer les oscillations de cette longue lance, on l'entoure d'une gaine de roseau, ou bien 1'on attache à la lance une hampe de bois léger an moyen d'un fil de fer qui en assure la rigidité.

Afin de diminuer notablement les frais considérables occasionnés par 1'action dissolvante qu'exerce le liquide sur les tubes de caoutchouc, il faudrait munir les pulvérisateurs de tubes dits "cirés ", pourvus d'une spirale intérieure en acier et que naturellement il faut fabriquer exprès. Leurs extrémités doivent avoir en effet un anneau ou repli de la même substance, sur lequel on enfile et on lie fortement le morceau de tube de caoutchouc (résistant, à trois toiles) destiné à joindre la pompe au tube aspirant, ou au tube foulant "ciré " ou bien encore à la lance.

Pour faire fonctionner le pulvérisateur, on verse une petite quantité du liquide qu'il contient sur un peu d'herbe ou de paille sèches et l'on I met le feu; on présente alors à la flamme le jet de 1'appareil, et le pétrole benziné qui sort du pulvérisateur sous forme d'un nuage prend feu 
tout d'un coup; la flamme ainsi obtenue est prête à être dirigée sur les insectes.

Lorsque ceux-ci se sont groupés d'eux-mêmes en grand nombre sur une superficie restreinte, cette méthode donne des résultats rapides et très satisfaisants. Dans le cas contraire, des équipes de femmes et d'enfants composées de dix à seize individus, sont disposées en une ligne courbe ou en cercle selon que le point choisi pour y concentrer les orthoptères se trouve borné par un obstacle opportun ou que 1'on opère dans un champ ouvert; les rabatteurs traînent sur le sol une poignée de longues herbes ou mieux de branchettes, obligeant ainsi, à mesure qu'ils avancent et se rapprochent, les insectes qui étaient éparpillés à se concentrer vers le point indiqué. Alors d'autres ouvriers munis de pulvérisateurs remplacent les premiers et dirigent la flamme sur la masse des insectes préalablement rabattus.

Si 1'on se trouve en présence d'une apparition intense et étendue et que les orthoptères soient adultes, il y a grand avantage à travailler même la nuit. Dans ce cas, on éclaire le terrain au moyen de lampes à acétylène munies de réflecteurs.

Ia méthode de la flamme de pétrole-benzine est d'une efficacité incontestable et elle est vraiment pratique au point de vue de l'application - avec I hl de liquide on peut brûler de 250 à $350 \mathrm{~m}^{2}$ de terrain recouvert d'insectes - mais elle est loin d'être aussi économique - I hl du mélange coûte en moyenne 5o fr environ transporté sur les lieuxet d'autre part elle n'est pas exempte de danger pour les ouvriers qui doivent l'appliquer.

En Autriche, dans les prairies et les pâturages permanents du Carso gorizien, on extrait du sol, au moyen de hersages, pendant l'automne ou dans les mois de mars et d'avril, les coques ovigères qui sont amoncelées et brûlées sur place.

Dans la même région on a obtenu d'excellents résultats contre ces orthoptères, durant les mois de juin et de juillet, en organisant systématiquement leur capture à l'aide de petits collecteurs à main, à ouverture quadrangulaire et aux angles arrondis, semblables pour le reste aux filets à papillons bien connus, et confiés à des enfants; ou bien moyennant de grands collecteurs formés avec des sacs de toile cousus sur des châssis de fer rectangulaires et qui, soutenus par deux enfants, sont traînés rapidement sur le sol. Les sacs des deux types de collecteurs se rétrécissent en forme de cône au fur et à mesure qu'ils s'éloignent des châssis et ils se terminent par une petite ouverture fermée avec une ficelle.

Les insectes capturés à l'aide de ces appareils sont versés dans des récipients-réservoirs formés par des bidons à pétrole munis à leur partie supérieure d'une ouverture qui se ferme au moyen d'un couvercle à coulisse et percé de trous. Après avoir secoué le collecteur pour faire tomber les insectes au fond du sac, ou dénoue la ficelle qui ferme l'ouverture placée à son extrémité et on l'introduit dans le récipient-réservoir pour y faire tomber les insectes. Quand le récipient est plein, on verse de l'eau bouillante à travers les trous du couvercle pour tuer les insectes. 
Pendant l'été de I909, on a capturé et détruit en tout, à l'aide de cette méthode, environ rog 000 litres d'insectes, ce qui équivaut à peu près à une charge de II wagons et représente en chiffre rond 45000000 de ces insectes. Il résulte d'une enquête effectuée en IgIo, que la méthode suivie l'année précédente avait été aussi utile pour l'année suivante, car 1a quantité d'insectes était devenue insignifiante.

En Dalmatie, on a procédé à la recherche et à la destruction des foyers de ponte, opérations difficiles et pénibles, étant donnée la nature montueuse du sol.

La capture à l'aide de collecteurs a donné de bons résultats, surtout dans les terrains plats.

Mais la méthode de lutte qui a paru la meilleure dans la pratique est la suivante: un bon nombre d'ouvriers forment une large chaîne en demicercle et poussent les insectes avec des rateaux et des balais jusqu'à un point indiqué ; après quoi les insectes sont arrosés de pétrole au moyen d'un pulvérisateur type Vermorel et ensuite brûlés.

Le procédé de lutte élaboré et employé sur une vaste échelle en H ongrie depuis I907 - à la place des appareils "cypriotes " trop coûteux et d'ailleurs insuffisants pour combattre ces orthoptères dans les conditions du pays - consiste dans l'emploi d'une brosse d'acier, d'une longueur de 2 à $5 \mathrm{~m}$, traînée par deux chevaux. L'idée de cette machine a été tirée de la herse d'épines dont on se sert en Hongrie, dans la Russie méridionale et un peu partout sur les sols légers; on avait même parfois employé cette herse à titre d'essais en Hongrie et en Russie pour détruire ces orthoptères, sans que cette pratique devînt universelle.

L'avantage que présente la brosse en acier sur la herse d'épines vient de son poids et de sa résistance. Ia brosse repose sur des roues, ce qui permet aux insectes de s'introduire dessous. Les deux chevaux de paysan que l'on attelle le plus souvent à cet appareil avancent en marchant sur le côté et poussent les orthoptères soit sous la brosse qu'ils tirent soit des deux côtés de manière que les insectes échappés à la première brosse viennent sous celles qui suivent immédiatement de côté et en arrière. Les brosses échelonnées de la sorte écrasent et déchiquètent les insectes ou les blessent si bien qu'ils périssent bientôt.

En I908, 1a manœuvre a eul lieu avec 275 brosses : le travail commença tous les jours à 4 heures du matin, se poursuivant sans interruption jusqu' à 8 heures du soir avec des relais toutes les 2 heures. Le travail ne fut interrompu que pendant une demi-heure lorsque tombaient de fortes averses. Les petites pluies n'obligeaient pas à arrêter le travail. Plus tard, quand les insectes avaient pris les ailes, la pluie, le froid, le vent facilitaient le travail, parce que les sauterelles se remuaient difficilement et ne s'envolaient pas. Et même alors, vers la mi-juin, il ne fut possible de procéder avec succès contre les ailés que pendant les premières heures du matin et dans le soir.

La capacité de travail de cet appareil a été en moyenne de 8 arpents cadastraux $(4,6037$ ha), minimum sur lequel ou put compter avec certitude. Les frais des 8 arpents cadastraux nettoyés en un jour revenaient à 
25-26 couronnes $(26,25-27,30 \mathrm{fr})$ tout au plus, ce qui équivaut à une moyenne de 3,30 couronnes $(3,47 \mathrm{fr})$ par arpent cadastral $(0,57546 \mathrm{ha})$ alors que les frais des appareils cypriotes s'élèvent à 40-42 couronnes (42-44, Io fr) par arpent cadastral.

Plus tard, on prépara des brosses plus résistantes et dont certains détails furent améliorés. Ces appareils nouveau modèle reviennent maintenant à 300 couronnes ( $3 I_{5} \mathrm{fr}$ ) chacun; ils sont réunis dans un dépôt près de Debreczen à l'Académie nationale hongroise d'Agriculture et toujours prêts à entrer en activité à n'importe quel moment.

Le I6 juin I909, on signala à Nagikörü (comitat de Jásznagykúnszolnok) une apparition d'orthoptères s'étendant sur 700-80o arpents cadastraux $(402,822-460,368$ ha). La lutte commença le 2 I et le 22 du même mois, c'est-à dire à une époque où une partie des insectes avaient déjà pris les ailes. A cause de la trop grande chaleur, on ne put rien faire pendant le jour, les sauterelles volant de toutes parts, et il fallut se résigner à travailler pendant la nuit. Pour éclairer le terrain, on se servit de vieilles fascines de saule qu'on trouva en quantité suffisante le long de l'endiguement de la Tisza, et on appliqua aux appareils des torches enduites de poix. Bien que le travail fût nouveau et l'éclairage fort primitif — ce qui rendait difficile la marche des deux groupes composés de 50 appareils chacun - 1'essai réussit parfaitement et démontra qu'on peut combattre, avec la certitude absolue du succès, la sauterelle qui fait tant de ravages en Hongrie, qu'i1 s'agisse des jeunes insectes ou des ailés arrivés à leur complète évolution. Aujourd'hui tous les appareils sont munis de lampes et l'on dispose encore d'un nombre suffisant d'autres lampes qui servent à éclairer le terrain et à donner des signaux aux ouvriers, ce qui leur permet de s'orienter, et à diriger sans difficulté les travaux.

Une brosse à sauterelles peut travailler pendant 24 heures sans interruption, pourvu qn'on puisse relayer les attelages toutes les 2 heures, ce qui n'est pas difficile en Hongrie. Il faut compter 3 relais pour chaque brosse ( 6 paires de chevaux). Tant que les insectes n'ont pas d'ailes, on travaille jour et nuit sans s'arrêter ; dès que les ailes ont poussé, on ne travaille que de 7 ou 8 heures du soir à 6 ou 9 heures du matin.

La brosse est un moyen sûr et précis pour détruire ces insectes, étant données les conditions spéciales du sol hongrois et les moeurs de l'espèce de sauterelle nuisible en Hongrie. Car il ne faut pas oublier que cet appareil ne trouve son application que sur un terrain plat. Si le terrain est couvert de nombreuses plantes, sa capacité est sensiblement entravée car on ne saurait exterminer les insectes sans anéantir en même temps la végétation environnante.

Voici maintenant les moyens de lutte qui ont donné les meilleurs résultats et qui sont encore en usage en $\mathrm{R}$ o $\mathrm{um}$ a $\mathrm{n}$ i e.

Lorsque les surfaces de terrain reconnues comme infestées par les coques ovigères - on se sert pour les déterminer de la carte générale dressée par le Service entomologique central — sont relativement restreintes, et que le nombre des habitants des communes environnantes suffit pour assurer le travail, on fait la récolte des coques ovigères à la main; le prix 
que l'on en donne varie entre I2-20 fr l'ha. Si les surfaces sont trop grandes, on aime mieux faire det1x labours, l'un en automne et l'autre au printemps, et mettre ainsi à nu les œufs qui se dessèchent au soleil.

Lorsque les larves sont toutes jeunes, on place, de distance en distance, des tas de paille sur lesquels les larves se retirent le soir pour y passer la nuit. Le lendemain matin, de bonne heure, on met le feu à ces tas et l'on détruit ainsi une grande quantité d'insectes.

Si l'éclosion prend de trop grandes proportions, on entoure le lieu de ponte d'un fossé de $60 \mathrm{~cm}$ de largeur sur 50-70 cm de profondeur. Au fond du fossé, à une certaine distance les uns des autres, on pratique des trous de $40-50 \mathrm{~cm}$ de côté et d'autant de profondeur, appelés trous de loups. Des ouvriers armés de balais commencent à pousser les larves petit à petit vers les trous. Sur le chemin, avant d'arriver au fossé, d'autres ouvriers étendent de la paille en demi-cercle devant le front des insectes. Lorsque ceux-ci sont tout près de la paille, on $y$ met le feu et l'on s'efforce de balayer rapidement le plus que l'on peut d'insectes vers les flammes. De la sorte, après avoir détruit par le feu un grand nombre de larves, on arrive peu à peu jusqu'au bord du fossé où l'on précipite le reste. Alors des ouvriers écrasent avec des maillets les larves entassées au fond des trous de loups. Lorsque le fossé est presque plein, on y verse de la chanx, de la créosote, ou même du pétrole, mais celui-c1 avec beaucoup de réserve à cause des dangers qu'il présente.

Lorsque les larves sont plus âgées, on emploie presque exclusivement les fossés, rarement le feu. En ce cas, les fossés sont creusés en demi-cercle; devant le front de l'invasion on fauche assez ras toutes les cultures qui séparent les larves du fossé, afin de permettre aux ouvriers de les y pousser plus rapidement et en plus grand nombre. On creuse un second fossé parallèle au premier pendant que celui-ci se remplit.

Enfin, lorsque les adultes apparaissent, si le vol ne s'est pas encore posé à terre, on cherche à l'en empêcher en faisant le plus de bruit possible. Si le vol vient de s'abattre sur les cultures, comme les sauterelles sont ordinairement fatiguées après un long voyage, on les pousse vers des fossés que l'on prépare en toute hâte. On pratique bien aussi le roulage mais, à ce moment-là, la lutte est bien difficile à entreprendre.

En Serbie, dans le cours de l'année r 849 - époque où les sauterelles firent dans le pays leur première apparition en masse - un vol compact s'abattit sur le village de Korman (dép. de Kragujevac). Dès que les paysans aperçurent les insectes, ils se mirent à frapper sur des chaudrons, à tirer des coups de fusil, à pousser des cris, etc. et réussirent ainsi à chasser les sauterelles qui étaient descendues dans leurs champs. Le vol se leva pour aller s'abattre de nouveau dans les villages avoisinants et y produire des dégâts considérables.

En Igo6 au contraire, lorsque des orthoptères dont les ailes n'étaient pas encore complètement développées, apparurent en très grand nombre entre les villages de Vinci et de Požezena (dép. de Pozarevac), on creusa des tranchées parallèles au front de la bande en marche et l'on y poussa les 
insectes, après quoi on les y enterra. De cette façon, une grande partie de ces insectes furent détruits et les dégâts localisés.

Des divers procédés mécaniques et physiques expérimentés en Gr èce en IgII, ceux que nous décrivons ci-après ont été reconnus les plus pratiques et on les a appliqués l'année suivante.

Après avoir travaillé le sol à une faible profondeur, on s'est mis à la recherche des œufs et on les a ramassés à la main. On en a recueilli ainsi plusieurs milliers d'ocques ( $\mathrm{I}$ ocque $=\mathrm{I} 280 \mathrm{~g}$ ) soit par un travail obligatoire soit par primes ou par rétributions.

On a pratiqué aussi la destruction des insectes par le feu. Pour cela des ouvriers, à l'aide de branchettes qu'ils agitent près de la terre, chassent et rassemblent les insectes sur des matières inflammables, paille ou herbes sèches, auxquelles on met ensuite le feu. Ce procédé a donné de bons résultats dans les localités où 1'on pouvait se procurer abondamment ces matières.

On a essayé encore de détruire es orthoptères par assommage en les frappant avec des branches très fournies ou des balais en fils d'acier faits exprès. Cette méthode a été surtout employée par les maîtres d'école et leurs élèves et a donné d'excellents résultats. On 1'a appliquée la matin ou le soir, lorsque les insectes étaient réunis en masse, engourdis et presque immobiles.

Enfin, le ramassage a été fait en employant la méthode des toiles déjà citée pour d'autres pays. Ces toiles ont une longueur de 3 à $12 \mathrm{~m}$ et une largeur de 3 à $4 \mathrm{~m}$; elles sont munies, au milieu, d'une fente à laquelle est adapté un sac; une moitié de ces toiles est étendue sur le sol devant les insectes, l'autre moitié est relevée et soutenue par des ouvriers de manière à former une espèce de barrage vertical. La destruction des orthoptères capturés peut s'opérer en les écrasant ou en les enfouissant ou mieux encore en les plongeant, si possible, dans l'eau bouillante. Un simple drap sans ouverture médiane rend les mêmes services si 1'on a soin d'étourdir les insectes en les battant contre le sol. Par ce moyen plus de 250000 ocques $(320050 \mathrm{~kg}$ ) d'insectes ont été détruites, la plus grande partie contre payement.

Au Ja pon, pour la lutte contre le Locusta migratoria sont en usage différents moyens qui consistent : a) à creuser le sol et à en retirer les œufs qui s'y trouvent cachés ; b) à capturer les larves au moyen d'un filet ou à les détruire en les frappant avec des appareils appropriés; $c$ ) à écraser les insectes sur le sol avec un rouleaú; $d$ ) à brûler les herbes des champs infestés ; e) à employer des instruments de chasse.

Pour combattre 1'Oxya velox, qui est le fléau le plus redoutable de la culture du riz au Japon, on bêche, au printemps, les bordures de rizières, les fossés ou digues et les rizières elles-mêmes pour détruire les œufs qui s'y trouvent. En outre, comme les œufs enfouis dans le sol viennent généralement flotter à la surface de l'eau quand on travaille dans les rizières au printemps, on s'empresse de recueillir ces œufs afin de les détruire. 
En $\mathrm{C}$ h i n e, on profite des nuits les plus sombres pour brûler du bois ou des broussailles au bord de grands fossés creusés d'avance; les insectes réparidus dans les champs volent vers la lumière: une partie, brûlée par les flammes, tombe d'elle-même dans le fossé; les autres, qui n'ont pas été touchés par le feu, s'abattent et s'entassent près des tranchées où des ouvriers les poussent avec des balais.

Lorsqu'il s'agit de combattre des insectes qui ne volent pas encore, on procède comme il suit : on creuse, d'un côté du champ, un fossé d'une profondeur d'un "tchi » $(0,32 \mathrm{~cm})$; mais de place en place, au fond de ce fossé, on creuse encore de grands trous; le fossé lui-même est plus large à la partie inférieure qu'à la partie supérieure; des ouvriers tenant une espèce de bande de toile en bambou flexible se placent sur les trois autres côtés du champ et peu à peu, doucement, ramènent les insectes vers $1 \mathrm{e}$ fossé. Là on les écrase d'abord, puis on les recouvre d'une couche de chaux et de terre.

Ce moyen ne saurait être appliqué dans les champs cultivés. Dans ce cas, on agite de très longues baguettes flexibles et on bat le sol entre les plates-bandes occupées par la végétation. L,es insectes effrayés cherchent à se sauver; on les dirige alors vers quelque champ inculte où l'on emploie la méthode décrite tout à l'heure.

Il existe aussi pour capturer les ailés un appareil spécial formé de deux sacs de toile, de grandeur différente, rentrant 1'un dans l'autre et fixés à un même cercle de fer. Le sac interne se termine par une ouverture étroite; le sac externe est beaucoup plus large que l'autre ; tout l'appareil est supporté par une longue perche de bambou, et l'on s'en sert comme d'un filet à papillons. Les insectes pénètrent dans le sac interne, puis de là dans le sac externe d'où ils ne peuvent plus sortir. Cet appareil donne sans doute de bons résultats, mais il ne peut servir pour attraper une grande quantité d'insectes.

Dans les Etats Fédérés Malais, on emploie ainsi un moyen mécanique de lutte, mais, contrairement à ce qui eut lieu au début de la campagne, on l'applique à présent seulement d'une manière limitée, étant donnés les frais considérables qu’il entraîne, la détérioration rapide de l'appareil nécessaire et son inefficacité en présence des exigences de nombreuses localités ; on n'use de ce moyen que dans les zones où la méthode de l'empoisonnement présente un certain danger. Il consiste d'après la description qu'en font MM. H. - C. PRAT'T et F. DE LA MARE NorRIs, du Département de l'Agriculture - à pousser les insectes encore jeunes dans des enceintes ou barrages placés à une certaine distance du front d'une bande en marche, et disposés généralement de manière à former une série de $\mathrm{V}$ ou bien à affecter la forme d'un polygone irrégulier, avec des trappes spéciales à tous les angles.

Ces trappes sont facilement transportables, elles évitent le creusage de fosses ou de tranchées, elles sont plus efficaces que ces dernières pour empêcher la fuite des insectes lorsqu'ils y sont entrés et peuvent être placées en quelques minutes. Ce sont des collecteurs rectangulaires en toilé résistante, plutôt brune que blanche, ayant la forme de caisses sans 
couvercles. Le côté destiné à l'entrée des insectes est à tue hauteur de $60 \mathrm{~cm}$ environ. et cenx-ci y sont conduits au moyen d'un plan incliné formant un carré de I,20 m de côté, ce qui est également la largeur de la trappe. I,es deux autres côtés et la paroi postérieure ont une hauteur de I,35 m; les deux côtés sont prolongés par deux ailes de I,20 $\mathrm{m}$ de long, attachées dans le bas au plan incliné. $\mathrm{I}_{1}$ e tout est soutenu par des piquets de fet munis à leur extrémité de crochets auxquels la trappe est suspendue par des anneaux d'acier cousus à la toile. Le même moyen sert à soutenir et à tendre les ailes. La forme de la trappe est maintentie par quatre bambous qui passent dans des replis de la toile à $60 \mathrm{~cm}$ du sol, et placés à la partie extérieure de la trappe. Ces bambous sont maintenus par des piquets de fer munis à leur extrémité d'anneaux à travers lesquels ils passent. Du bambou qui soutient 1'entrée de la trappe, le plan incliné descend vers le sol, maintenu dans sa position par un autre bambou placé entre un repli de la base et fixé au sol au moyen de fers courts ou d'autres piquets. Pour s'opposer à la fuite des insectes par le dessous de la trappe, on jette de la terre à la base des ailes et sur le devant du plan incliné.

La sortie des insectes est empêchée par des bandes de 'toile cirée de I5 à $30 \mathrm{~cm}$ de large, cousues autour du bord intérieur de la trappe, sur le devant au niveau de 1'entrée, et sur les côtés et la paroi postérieure I $5 \mathrm{~cm}$ plus haut. Une autre bande est cousue à environ $30 \mathrm{~cm}$ du bord supérieur autour de la paroi postérieure, sur les côtés et le long des ailes. I,es côtés du barrage en $V$ sont de forte cotonnade d'une longueur de $13,5 \mathrm{~m}$ environ et d'une largeur de I,35 m. Du côté intérieur deux bandes parallèles de toile cirée, larges de $\mathrm{I} 5 \mathrm{~cm}$, sont cousues à une hauteur de $90 \mathrm{~cm}$ et de $\mathrm{I}, 20 \mathrm{~m}$ de la base. A 1'extrémité est attachée une corde solidement cousue avec de la ficelle à des intervalles de $2,5 \mathrm{~cm}$. Les toiles latérales sont soutenues par des piquets de fer d'une longueur de I,35 m, munis d'un crochet sur lequel est enfilée la corde; elles sont maintenues dans le bas par une ligne ininterrompue de terre. Quatre cents mètres de cet engin peuvent être placés devant une bande en marche par dix coolies adroits en moins d'une demi-heure. De plus, il est facile, lorsque la bande se trouve dans l'enceinte, de lui couper la retraite en fermant l'extrémité ouverte du V ou de la figure formée par la disposition donnée au barrage. Les insectes restent alors prisonniers et, quelle que soit la direction dans laquelle ils se meuvent, ils finissent toujours par arriver à une des trappes. Le système est rendu souvent plus efficace en introduisant des toiles de division qui vont de la base d'un $V$ à la trappe. Cela tend à éviter que les insectes ne commencent à tourner en rond à proximité du sommet de l'angle, ce qu'ils font souvent lorsque ce dernier est large.

Le succès de ce système de lutte dépend beaucoup du placement judicieux de la trappe. On a observé, à ce propos, de nombreux cas où 1'appareil ayant été placé sur la ligne suivant laquelle les insectes avançaient sans les déranger, ceux-ci ont continué à se diriger, comme un courant régulier et ininterrompu, vers les trappes pendant plusieurs heures et sont tombés dedans après avoir franchi le plan incliné, sans que les coo- 
lies aient eu à se donner grand'peine pour les diriger vers ces trappes. Quand le nombre de ces dernières est insuffisant, il est souvent utile de les remplacer en creusant une fosse profonde de 60 à $90 \mathrm{~cm}$.

La destruction des insectes réunis dans la trappe peut se faire de différentes manières. La majeure partie meurt par étouffement et par la pression exercée par les insectes les uns sur les autres. Le moyen le plus efficace et le plus économique de tuer les survivants, c'est de verser sur eux de la kérosène. Le piétinement, l'écrasement au moyen de battes et l'asphyxie par le sulfure de carbone ont également bien réussi.

De septembre IgI3 à novembre IgI4, on a détruit, en appliquant la méthode mécanique dans les Etats Fédérés Malais (Selangor et NégriSembilan) 99374 bidons à kérosène d'insectes non ailés, représentant 5I34 bandes. Puisque chaque bidon contenait 4 "gallons " (I8 litres), la quantité totale d'insectes capturés fut de I7 887,32 h1. En I9I5, on a capturé dans le Selangor I97 bandes équivalentes à 4633 bidons (834 h1) et dans le Négri-Sembilan 583 bandes correspondantes à 5522 bidons ( 994 hi).

La même méthode est employée depuis IgI4 dans 1'Etat de J o hor e, oì, du 24 novembre au $3 \mathrm{I}$ décembre de cette même année, on a rempli 378,5 bidons $(67,5 \mathrm{hl})$ équivalents à I79 bandes, seulement dans les deux districts de Lanadron et de Tangkah. Au cours de I9I5 on a capturé dans le Johore 437 bandes équivalentes à $\operatorname{rr5} S$ bidons (208,5 h1).

Le ramassage et la destruction des œufs, le labourage immédiat du terrain où les œufs ont été pondus, la capture des insectes non ailés dans des tranchées remplies d'eau ou, à son défaut, de paille à laquelle on met le feu dès qu'un grand nombre d'insectes y sont tombés, sont autant de moyens qui ont été reconnus très efficaces dans l'In de pour la lutte contre le Schistocerca tatarica et ce sont, dans les conditions présentes, les seuls qui peuvent être recommandés en toute sécurité à la population.

L'engin imaginé par M. H.-M. LEFROY pour la capture des jeunes orthoptères (Cyrtacanthacris succincta et Schistocerca tatarica) a fait preuve aussi d'une grande efficacité : un petit châssis de bambou est placé dans un sac dont une extrémité est ouverte et maintenue en cet état par ce châssis. Des cordes sont attachées à ce dernier et 1'appareil est traîné rapidement sur le sol. Les insectes entrent dans le sac dont on enlève de temps en temps le châssis, et les insectes capturés sont écrasés. Cet appareil est très léger et peut être manœuvré par une seule personne. Quand il s'agit de l'employer dans les endroits cultivés, on peut se passer du châssis, et le sac est tenu ouvert par deux morceaux de bambou. L'absence du châssis enlève tout danger d'endommager les cultures.

En Algérie et en Tunisie, pour détruire les pontes surtout dans les terrains de culture, ceux-ci sont piochés ou labourés très superficiellement. Pour que ce travail réussisse le mieux possible, il doit être effectué immédiatement après la ponte. Ces façons peuvent faire avorter 1'éclosion dans la proportion de 50 à $80 \%$.

Comme nous l'avons déjà dit ailleurs, 1'Administration tunisienne distribue, dans certaines circonstances, aux indigènes une prime fixe 
par "guelba ") (20 litres) d'œufs ramassés à la main ; on met les œufs dans des sacs ou des coufins, puis on les entasse et on les brûle en les arrosant de pétrole ou de goudron.

On a recours, contre les larves (criquets) très jeunes, à l'écrasement fait de bon matin au moyen de branchages, de balais, de morceaux d'étoffe, etc. avec lesquels on bat le terrain occupé par ces orthoptères.

Plus tard, lorsque les criquets se réunissent en bandes et se mettent en marche, on les conduit sur des chaumes ou des broussailles isolés et on les brûle. Mais cette méthode est dangereuse, surtout à cause des incendies qu'elle peut provoquer.

On creuse aussi, au bou moment, des tranchées ou des fossés de faible profondeur, on y refoule en masse les jeunes insectes et on les détruit en les écrasant ou en comblant les fosses dès qu'elles sont pleines.

On se sert aussi des pièges Ortel, qui portent le nom de leur inventeur; celui-ci imagina cette méthode de lutte en observant les mours des jeunes insectes. I orsqu' ils se mettent en marche, ils choisissent surtout les pisțes ou les sentiers faciles, libres d'herbe et de tout obstacle en général, ils descendent les pentes plutôt qu'ils ne les remontent et ont toujours une tendance à se réunir en groupes serrés et à suivre tous la même direction lorsque la marche est commencée.

Or le procédé Ortel consiste à tracer dans les lieux de ponte un réseau de petits sentiers rudimentaires aplanis, disposés de manière à former sur le sol une série de losanges au sommet desquels sont préparés des pièges où les insectes vont tomber en suivant les sentiers. Ces pièges consistent en fosses ou trous cylindriques de 40 à $45 \mathrm{~cm}$ de profondeur et de $60 \mathrm{~cm}$ de diamètre, dont la paroi est revêtue, dans sa partie supérieure, de deux bandes en zinc; ces dernières, qui ont I m de long et $35 \mathrm{~cm}$ de large, s'appliquent parfaitement à la paroi de la trappe en les recourbant à la main, et on les fait adhérer l'une à l'autre aux deux points de contact à l'aide de deux morceaux de bois nerveux fendus sur une partie de leur longueur. Les bandes en zinc, le long desquelles glissent les insectes arrivés sur le bord de la trappe, sont placées de manière à ne pas dépasser le niveau du sol et elles sont légèrement enduites d'huile sur le bord supérieur.

La bande des jeunes insectes est refoulée vers les fosses et lorsque celles-ci sont à peu près remplies, il ne reste plus qu'à écraser les insectes capturés ou à combler les trous.

Les indigènes de l'Algérie emploient aussi avec une adresse toute spéciale les engins appelés "melhafas». Un melhafa est une bande de toile blanche ou grisâtre, d'une longueur de ro $\mathrm{m}$ environ et d'une largeur de 3 à $4 \mathrm{~m}$, que l'on tend à moitié verticalement devant une bande de criquets en laissant l'autre moitié étendue sur le sol, comme cela se pratique pour les "tentes" ou toiles proprement dites dont nous avons déjà eu l'occasion de parler à plusieurs reprises pour d'autres pays. La différence entre le melhafa et la toile, c'est que cette dernière est généralement munie dans sa partie centrale d'une fente qui conduit dans un sac où les insectes vont s'accumuler; mais le fonctionnement des deux engins est essentiellement le même. Quant aux jeunes insectes qui, refoulés par une 
équipe de rabatteurs, vont se rassembler sur le melhata, on les détruit en les écrasant ou en les enfouissant.

Contre les bandes de criquets en marche, ou emploie aussi, depuis longtemps, les appareils cypriotes. Nous avons eu déjà l'occasion de mentionner ces appareils parmi les moyens mécaniques que nous avons décrits. Comme, contrairement à ce qui a lieu en Hongrie et dans l'île de Chypre, ces appareils sont encore en usage en Algérie et en 'Tunisie, le moment est venu de les décrire brièvement.

Iie système de lutte connu sous le nom de cypriote fut imaginé, dans la seconde moitié du XIXème siècle, par RICCARDO MATTEI, grand propriétaire d'origine italienne établi à Chypre; il a subi depuis des modifications et des perfectionnements variés. Il consiste en barrages que l'on place devant la bande en marche à détruire; ces barrages sont formés de bandes de toile d'une longueur de $50 \mathrm{~m}$ et de 80 à $85 \mathrm{~cm}$ de hauteur ; à leur partie supérieure et à l'intérieur, c'est-à-dire du côté qui est tourné vers les insectes, ces bandes sont ourlées d'une bande de toile cirée de Io à $x 5 \mathrm{~cm}$ de large et dressées verticalement sur le sol ou, mieux, un peu inclinées sur le front de la bande en marche; pour maintenir les toiles dans la position voulue, on se sert de piquets en bois ou en fer placés du côté opposé à celui qui se trouve vers les criquets et enfoncés dans le sol de trois en trois mètres ; ces piquets sont attachés aux toiles à l'aide d'une double ligature et reliés entre eux à leur extrémité supérieure au moyen d'une corde. I a toile est fixée aussi à cette dernière par une ligature placée sur son bord supérieur et à une distance égale des piquets voisins. On laisse traîner sur le sol environ $20 \mathrm{~cm}$ de toile sur toute sa longueur et on recouvre de terre cette partie de la toile de manière à la fixer et à boucher tous les passages.

Selon les conditions dans lesquelles on est appelé à opérer, on place les toiles en ligne droite, en demi-cercle, en lignes brisées ou, de préférence, de manière à former un large $\mathrm{V}$ avec l'ouverture tournée vers les insectes; pour mettre l'appareil en forme de dispositif vraiment typique, il ne reste plus alors qu'à creuser au sommet du $V$ une fosse de dimensions variables. S'il s'agit de combattre des invasions considérables, on prépare en outre le long de chaque branche du V et vers la moitié, une fosse supplémentaire semblable à la première. Après quoi, des équipes de rabatteurs munis de branchages obligent les criquets en marche à entrer dans l'espace limité par les toiles que les insectes ne peuvent pas franchir, car leurs pattes n'ont aucune prise sur la bande de toile cirée; ils retombent par terre, reprennent leur route et finissent par arriver sur les bords de la fosse dans laquelle ils se précipitent en désordre sans pouvoir en ressortir, car les bords en ont été préalablement revêtus de bandes en zinc, qui jouent pour les insectes le même rôle que la toile cirée. Lorsque la fosse est presque pleine, on détruit le plus souvent les criquets que l'on a pris en les écrasant ou en les brûlant.

Outre les appareils cypriotes. les barrages métalliques constituent un des moyens de lutte les plus employés en Algérie contre les criquets.

MM. CH. Rivik̀re et H. LECQ ont proposé, en I89I, pour capturer les 
jeunes criquets de Schistocerca tatarica, l'usage de bandes en zinc de $2 \mathrm{~m}$ de long sur $40 \mathrm{~cm}$ de haut et d'une épaisseur de $20 \mathrm{~mm}\left(\mathrm{n}^{\circ} 4\right)$ qui, juxtaposées l'une à l'autre, sont dressées sur le sol au moyen de piquets de fer et disposées, comme les toiles des appareils cypriotes, verticalement ou légèrement inclinées sur le front de la bande en marche. Les criquets refoulés par les rabatteurs, vont tomber dans les fosses préparées à une certaine distance l'une de l'autre à la base de la clôture et on les y détruit en le; écrasant.

Les barrages en zinc offrent certains avantages sur les appareils cypriotes. D'abord, ils coûtent moins cher; en effet, I km de ces barrages métalliques vaut $550 \mathrm{fr}$ au lieu de $777,50 \mathrm{fr}$; de plus, les bandes en zinc ne se détériorent pas comme les toiles, de sorte qu'elles valent encore 1 es $2 / 3$ du prix d'achat lorsqu'on s'en est servi et qu'on peut, si l'on veut, les employer à d'autres usages. Puis les barrages métalliques sont beaucoup moins lourds: $885 \mathrm{~kg}$ contre $1300 \mathrm{~kg}$, poids des toiles nécessaires pour former la longueur de I $\mathrm{km}$.

Enfin, en ce qui concerne la destruction des insectes ailés, on opère d'habitude l'écrasement de bon matin, alors qu'ils sont encore engourdis par lefroid, et cette opération peut encore se répéter lorsque les sauterelles sont accouplées ou occupées à la ponte. En Tunisie, les indigènes ramassent précisément les sauterelles pendant les heures froides du matin et même les soir et les mettent en sac. On leur fait des distributions de sel et ils utilisent ces insectes pour leur nourriture. Les quantités ramassées sont parfois considérables et se chiffrent par milliers de sacs de $50 \mathrm{~kg}$ (40000 dans le seul caïdat di Djérid en Igo8).

En E $\mathrm{ry} t \mathrm{~h} r$ é e, on emploie avec succès le battage des insectes aux premiers stades de leur vie.

Au Togo, les indigènes du district de Lome n’ont lutté jusqu'à présent que contre les jeunes insectes en creusant dans les champs de longues tranchées, qu'ils ont comblées dès que les insectes y sont entrés. Par contre ceux du district de Misahöhe ne combattent que les ailés, qu'ils ramassent pour se nourrir. Ils cherchent aussi à les éloigner des champs et des cultures au moyen de feux accompagnés d'une fumée très dense, ainsi qu'avec des cris et des bruits; de plus, ils cherchent à chasser les insectes qui s'approchent de terre en les frappant avec des branches.

Les indigènes de la Nigéria profitent de ce que les jeunes orthoptères se meuvent en bandes serrées et toujours dans la même direction pour les capturer en crensant des fosses profondes d'un mètre environ, devant la bande en marche; lorsqu'ils y ont refoulé les insectes, ils les détruisent en les ensevelisant ou en les brûlant.

Lorsque les indigènes, qui se nourrissent aussi de sauterelles, voient les adultes se poser, ils repèrent le point, et, dès l'aurore tout le voisinage sort pour les attraper et les tuer en grand nombre. Lorsque le vol n'est pas considérable, la population allume des feux qui produisent une épaisse fumée, bat sur des calebasses, des tambours, et refoule ainsi les sauterelles qui se sont abattues dans une localité donnée. D'ordinaire la population 
est avertie de l'approche du fléau. Pour chasser les ailés, les indigènes recourent aussi au moyen de les battre avec des branches.

Au Ca m e r o $\mathrm{n}$, toutes les fois que la présence des sauterelles a été constatée à Ngaundere et à Garua, on les a chassées en allumant des feux et en faisant un grand bruit.

L.es sauterelles sont un aliment apprécié par les indigènes du Congo belge, qui, aussitôt que des passages de ces insectes ont été signalés, se sont empressés de les recueillir pour les manger.

Dans la Province de Mozambique, les indigènes ont recours au procédé très primitif d'effrayer les vols par un tintamare infernal en frappant sur des ustensiles en fer-blanc.

Dans les plantations de canne à sucre de la région du Zambèze on combat le fléau plus sérieusement et avec de bons résultats en remuant la terre entre les files de cannes à sucre et en se livrant au ramassage des œufs. Ce procédé est pratique à condition, toutefois, que la main-d'œuvre soit à bon marché. Mais même ainsi les frais occasionnés par cette opération sont toujours élevés.

Dans la R ho désia méridiona 1 e, en plus du moyen chimique généralement employé contre les jeunes insectes, on brûle, lorsque la chose est possible, l'herbe sèche dans laquelle les bandes sont rassemblées.

On emploie aux $\mathrm{C}$ a $\mathrm{n}$ a $\mathrm{r}$ i es les mêmes moyens que ceux dont on use en Espagne.

Au Canada, lorsque arrive l'automne, on détruit les œufs en labourant la terre. Ce qui rend cette opération possible, c'est que les lieux de ponte occupent presque invariablement des terrains qui sont encore cultivés ou qui l'ont été récemment, tandis qu'on les trouve rarement dans les prairies incultes.

On emploie aussi le labourage pour détruire les jeunes insectes avant que leurs ailes n'aient poussé, ou bien on a recours au brûlage des insectes lorsqu'on est parvenu à les rassembler sur de longs tas de paille préparés d'avance en guise de pièges sur le sol infesté.

Toutefois la méthode mécanique la plus sûre contre ces orthoptères est offerte par 1'appareil appelé " hopper-dozer ». On trouve de nombreux modèles 'de cet engin, mais en général il consiste en un récipient peu profond: un plateau oblong detôle de fer - le plus souvent galvanisée et d'une épaisseur convenable - ayant les bords relevés tout autour, et monté sur des glissières ou des roues qui le maintiennent au-dessus du sol. Sur 1a partie postérieure de ce plateau est fixé un châssis vertical, de $60 \mathrm{~cm}$ de haut, et recouvert le plus souvent de toile. Le plateau est rempli d'eau à moitié; de la kérosène y surnage, et le tissu qui revêt le châssis postérieur est également imprégné de kérosène ou d’une autre huile minérale. L'appareil est traîné généralement sur le sol infesté par un ou deux chevaux, selon sa grandeur.

Une fois que l'appareil est mis en mouvement, une barre étroite, fixée à une courte distance sur le devant du plateau et au niveau du sol, 
oblige les insectes à sauter. Venant alors à se heurter contre le châssis ou à tomber sur le plateau, dans l'un et l'autre cas leur perte est assurée, car, par suite de leur contact avec l'insecticide, ils finissent toujours par périr, même s'ils réussissent à sauter hors de l'appareil.

Aux E t a t s-Unis, on s'en tient à peu près aux mêmes méthodes mécaniques qu’au Canada.

Pour la destruction des œufs les labourages sont utilement employés, car, même sans être très profonds, ils permettent d'enterrer et de recouvrir suffisamment les œufs pour empêcher les jeunes insectes de sortir au moment de l'éclosion.

Les travaux effectués, selon la nature du sol, avec des herses légères à dents ou avec des herses à disques, servent à rompre et à écraser un grand nombre d'amas d'œufs et à exposer les autres à l'action destructive des agents extérieurs.

Lorsque les insectes éclosent au milieu de la végétation morte, ils peuvent être détruits très économiquement et efficacement par le feu. Si la végétation sèche n'est pas suffisante, pour activer le feu, on répand sur la zone infestée du vieux foin, de la paille, etc.

On se sert également depuis longtemps, pour détruire les sauterelles à tous leurs stages, du plus jeune à l'adulte, de l'appareil connu sous le nom de "hopperdozer" ("hopper-dozer", "hopper dozer"). L'intérieur du plateau est presque toujours divisé, a11 moyen de cloisons, en compartiments dont le nombre varie suivant la grandeur totale de l'appareil; cela pour empêcher, lorsque le sol sur lequel on opère est en pente, que le liquide insecticide contenu dans le plateau ne soit trop battu et ne vienne à se répandre. Au lien de la kérosène, on emploie quelquefois le goudron.

Au Mexique, on a essayé de combattre les sauterelles à 1'aide de cris, de détonations d'armes à feu, de feux d'artifices, etc., mais sans obtenir un résultat satisfaisant.

Le creusage de fosses pour y capturer les insectes n'a donné un bon résultat que sur de petites étendues de terrain.

I,e meilleur des moyens employés consiste à pulvériser sur les bandes du pétrole brut à 1'aide de pulvérisateurs spéciaux et à enflammer ensuite les insectes arrosés. Le meilleur moment pour effectuer cette opération est celui où les femelles, occupées à la ponte, se trouvent dans l'impossibilité de fuir.

Pendant l'invasion de I9I5, les habitants de Costa-Rica, pour sauver leurs récoltes, cherchèrent plutôt, en général, à chasser les sauterelles qu'à les détruire. Aussi empêchèrent-i1s les vols, dès leur apparition, de se poser sur les champs, en les effrayant par des explosions de dynamite on de poudre, ou bien encore, si malgré cela les sauterelles parvenaient à descendre, en recommençant ces détonations au lever du soleil. Ces procédés adoptés presque partout ont eu pour effet de disperser les grandes bandes et de les séparer en groupes, importants encore et excessivement nombreux, qui ont couvert presque tout le pays.

Sur certains points cependant, le Gouvernement, qui avait organisé 
militairement 5 à 600 hommes, parvint à détruire les insectes. Les torches de feu furent un moyen de lutte des plus heureux, comme aussi de grands feux, la nuit, attirèrent et anéantirent quantité de ces insectes.

Lorsque, plus tard, le Gouvernement vit le fléau s'étendre sur tout le pays, il se décida à adopter la méthode du ramassage; et il paya comme on l'a déjà dit ailleurs - I "colon 》 $(2,40 \mathrm{fr})$, les Ioo livres $(46$ $\mathrm{kg}$ ) d'insectes; Ioo livres comprennent environ 25000 insectes. Le résultat a été excellent : hommes, femmes et enfants se sont mis en chasse et ont détruit de cette façon une énorme quantité d'orthoptères. Les frais de cette campagne ont paru mieux utilisés que les sommes consacrées à d'autres moyens, relativement moins importants, vu le résultat obtenu et surtout enfin bien répartis, sans déchets ni abus.

Dans 1'île de Saint-Christophe (St.-Kitts), on a combattu parfois ces insectes lors de leur apparition, en les ramassant à la main.

La méthode la plus souvent employée jadis à la Trinité, à l'époque des invasions, consistait à capturer les orthoptères et à les brûler.

Quand, en I886, le comté de Berbice, dans la G u y a n e a n $\mathrm{g} 1$ a i s e, fut gravement attaqué par ces insectes, ce fut encore le ramassage à la main qui fut le plus universellement adopté et qui réussit le mieux. Dans quelques localités, on eut recours aussi à des châssis garnis de toile, d'une longueur de I,20 à $\mathrm{I}, 80 \mathrm{~m}$ et de 0,60 à $\mathrm{I}, 20 \mathrm{~m}$ environ de largeur qui, enduits de goudron, furent passés en manière de brosse sur les bandes de jeunes insectes,; ceux-ci adhéraient au goudron, que l'on renouvelait de temps en temps.

Durant 1'apparition de I9I5, à Corentyne Coast, Berbice, on a ramassé ces orthoptères dans des bidons à kérosène et on les a brûlés dans des fosses profondes en les arrosant encore de kérosène.

Le ramassage des ailés, favorisé par l'état de torpeur dans lequel ils se trouvent le matin et par la quantité limitée des insectes; a donné jusqu'à présent au P é r o u les meilleurs résultats que l'on puisse attendre de ce procédé, étant donné le bon marché de la main-d'œuvre fournie opportunément par les indigènes.

Les principaux moyens mécaniques usités au B r é s i 1 sont les fosses, les barrages en zinc et les "vassouras de fogo ", appareils spéciaux à gazoline ou à benzine rectifiées; après avoir enflammé le liquide à sa sortie de l'appareil, on le dirige contre les insectes à détruire. De plus, on bat et l'on comprime avec des battes les terrains occupés par les pontes.

Dans la République Argentine, la destruction des œufs s'opère en remuant le sol qui les contient, surtout avec la herse à disques, et, à son défaut, avec la charrue ordinaire. Dans les endroits où l'on ne peut employer aucun de ces deux instruments, on se sert de la pelle ou de la bêche. On travaille la terre à une profondeur de 7 à $\mathrm{S} \mathrm{cm}$, de manière à exposer les cufs à l'action du soleil et de la pluie pour en éviter l'éclosion, ou inême plus profondément encore afin de bien enterrer les oeufs 
et d'empêcher ainsi les larves qui pourraient éclore de parvenir à la surface du sol. Ces travaux sont suivis d'un ou plusieurs ratissages qui, dans le premier cas, servent à diviser les tas d'œufs, et, dans le second, à combler les interstices qui ont pu rester dans le terrain travaillé.

Pour la destruction des pontes, on tasse aussi le sol infesté lorsqu'il est encore humide par suite de pluies récentes; on se sert pour cela de rouleaux, de herses formées de branches et chargées d'un poids lourd, et de gros bétail que l'on dirige de manière à le faire passer plusieurs fois sur les endroits où les œufs ont été pondus. L,es mêmes moyens (rouleaux, herses, bétail) sont employés aussi pour détruire les insectes très jeunes (stade de "mosquita ") ou déjà un peu plus développés (stade de "saltona ").

Pour combattre ces insectes dans les prairies naturelles et artificielles, depuis les 8 ou ro premiers jours qui suivent l'éclosion jusqu'au moment de la métamorphose, on a beaucoup généralisé dans les terrains plats l'usage d'un appareil très simple, un collecteur qui porte le nom de "carcarañá ", et que chaque agriculteur construit lui-même. Il se compose de deux châssis de bois rectangulaires reliés entre eux le long d'un des côtés les plus longs de manière à former entre les deux plans un angle de $45^{\circ}$ maintenu ouvert au moyen de deux règles de bois fixées aux deux extrémités libres des châssis. I’armature ainsi formée est entièrement reconverte de toile ou de bandes de zinc, sauf du côté opposé an sommet de l'angle, qui constitue l'ouverture du collecteur. On traîne celui-ci sur le sol, au lever et au coucher du soleil, à l'aide de deux chevaux qui marchent de chaque côté de l'appareil. La machine recueille ainsi une grande quantité d'insectes que l'on enterre en versant le contenu du collecteur dans des fosses creusées à l'avance.

L'usage des barrages en zinc (rouleaux de ro $\mathrm{m}$ de longueur sur 40$45 \mathrm{~cm}$ de largeur, dont la Direction de 1'Agriculture et de la "Defensa Agrícola " a une provision de plus de 20000 ooo de m) est de tous les systèmes le plus répandu et donne les meilleurs résultats contre la "saltona "; ce moyen de destruction est employé également contre l'orthoptère à l'état de "mosquita "; les bandes de l'insecte s'y trouvent enfermées et y meurent d'inanition après un certain temps.

Lorsque la "saltona " commence à procéder par groupes, on dispose les barrages en deux lignes convergentes, et l'on creuse contre elles de loin en loin plusieurs fosses dont on couvre partiellement l'ouverture avec dela tôle de fer galvanisé à surface lisse sur laquelle on répand de la terre: on a là des trappes où tombent les insectes. Les enceintes que forment ces barrages remplacent avantageusement les tranchées, car il faut beaucoup moins de temps pour les installer et il est plus facile de les vider et d'y recueillir ainsi successivement une plus grande quantité d'insectes.

Quand il s'agit de cultures importantes menacées par le fléau (surtout des cultures tardives de maïs et de lin) entourées de champs incultes, on fournit aux intéressés une quantité suffisante de barrages pour entourer ces cultures, de sorte qu'on les munit d'un vrai capital de matériel de défense contre payement, comme nous l'avons dit autre part, d'une somme très modeste. Au moment de la livraison des barrages né- 
cessaires, les agriculteurs doivent pratiquer à une certaine distance des lignes formées par ces barrages, un nombre assez grand de fosses ou d'enceintes pour capturer les bandes qui les côtoient, de sorte que le matériel ne sert pas seulement à protéger les terrains ensemencés, mais qu'il contribue aussi à la destruction du fléau.

Lorsqu'une zone inculte envahie se trouve à proximité d'une zone cultivée indemne, on installe des lignes fixes de défense générale avec des barrages en zinc et l'on forme avec ces barrages les enceintes dont il s'agit. Quelques-unes de ces lignes arrivent à avoir une étendue considérablejusqu'à $60 \mathrm{~km}$ - et l'on y détruit des multitudes énormes de "langosta ».

Pour anéantir la "mosquita » et la "saltona", on emploie également le feu, que l'on met à des chaumes de blé ou de lin et même à du fourrage précédemment coupé, que l'on distribue autour des bandes ; on en- flamme quelquefois aussi du naphte pulvérisé, ce qui provoque une explosion qui tue les insectes sur un assez vaste rayon; enfin on remplit de ce même liquide inflammable des appareils spéciaux à pression d'où jaillit une langue de feu de 60 à $80 \mathrm{~cm}$ que l'on promène sur les bandes.

Jusqu'aux dernières invasions on s'est livré, au $\mathrm{P}$ a $\mathrm{r}$ a g $\mathrm{t}$ a y, à la destruction de ces insectes en les frappant, ou en les brûlant ou en creusant des tranchées pour les capturer; les deux premiers moyens peuvent produire debons résultats si la lutte est bien dirigée, si elle est entreprise à temps et si 1'on y occupe un nombreux personnel. Mais le moyen qui réussit le mieux est assurément celui des barrages portatifs qui n'a été expérimenté qu'en dernier lieu.

Les barrages métalliques sont un des moyens mécaniques les plus

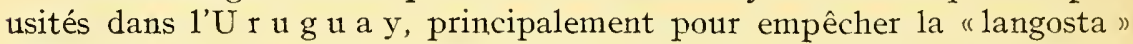
de se répandre dans les champs cultivés et rendre ensuite sa destruction plus facile et, en second lieu, pour tuer directement l'insecte ; car on resserre ainsi une bande de "saltona " entre les lignes de barrages, et on l'y laisse mourir de faim.

Les fosses, bien qu'employées rarement seules, peuvent servir cependant à la destruction de la "langosta "; elles sont, par contre, presque indispensables pour unir deux lignes de barrages.

Plusieurs types d'appareils à pression présentent aussi une grande utilité ; ils fonctionnenent à 1'aide du naphte, dont la flamme peut détruire une importante quantité d'insectes. Malheureusement cette méthode est une des plus coûteuses, non seulement à cause de la valeur intrinsèque de 1'appareil, mais aussi à cause du prix du combustible.

On répand parfois sur le terrain infesté de la paille de blé passée à la batteuse et l'on y met le feu; si la "langosta " se trouve dans un champ de fourrage déjà sec, on incendie le champ. Ce procédé est à la fois économique et efficace.

Il y a encore d'autres moyens de lutte, mais secondaires. Citons d'abord les "látigos ", sortes d'instruments formés d'une toile métallique ayant à peu près $60 \mathrm{~cm}$ de long sur $30 \mathrm{~cm}$ de large et fixée à un manche de bois au moyen duquel ou frappe sur les insectes comme avec un balai. Au lieu de cette toile métallique on fait usage aussi dè petites chaînes métal- 
liques tressées. On se sert encore de herses spéciales traînées par des chevaux ou par des mules, mais uniquement dans des terrains très plats et où l'on ne rencontre, en fait de végétation, que celle des pâturages naturels.

Dans certains cas, on fait courir sur le sol des juments et des brebis en formant autour des bandes un cercle de plus en plus étroit, de manière à écraser les insectes sous un piétinement ininterrompu.

En Australie, et particulièrement à Victoria Mill dans le $Q$ u e e n s1 a n d, les sauterelles causèrent en I 883 de grands dommages à la canne à sucre; dans la campagne qui suivit, on obtint d'excellents résultats en refoulant les jeunes insectes dans des fosses profondes de $60 \mathrm{~cm}$ environ, creusées autour des zones occupées par ces orthoptères.

En I904, la "Colonial Sugar Company " employa avec succès la méthode des barrages et des fossés contre le Locusta australis, qui menaçait les plantations de canne à sucre de Childers, dans le Queensland méridional. On eut encore recours à de la mélasse dans laquelle on poussa et 1'on asphyxia un grand nombre de jeunes insectes.

Dans la Nouvelle-Galles du Sud, entre autres moyens conseillés, on adopta dans des cas particuliers le feu dans les lieux de ponte au moment de l'éclosion ainsi qu'un rouleau pesant contre. les jeunes insectes en marche.

Dans 1'Etat de Victoria, le hersage, le labourage, l'irrigation, le foulage, le ramassage ont servi, avec plus ou moins de succès, à la destruction des œufs. Le hersage réussit fort bien en automne ou, à l'entrée de l'hiver, par un temps doux et sec; il prévient des dégâts ultérieurs. Une herse tournante ou un cultivateur peut rendre d'excellents services non seulement dans les champs, mais aussi le long des routes et dans des lieux nus et incultes. Le but est de remuer et d'émietter le sol à une profondeur d'environ $4 \mathrm{~cm}$. Des herses de branchages ou à chaîne, des rouleaux, etc., tuent ou blessent la majeure partie des insectes.

Un grand succès a été obtenu par le système de faire piétiner les jetnes orthoptères par des troupeaux de bœufs, de chevaux ou de moutons.

Une légère bande de fer-blanc luisant, large de plusieurs $\mathrm{cm}$ et placée autour d'un arbre, empêche les insectes d'y grimper et d'en dévorer les feuilles.

\section{MOYENS CHIMIQUES}

En France (Camargue), on a employé pour la destruction des jet1nes insectes du monosulfure de sodium dilué dans sept ou huit fois son poids d'eau.

Au Portuga1, on s'est servi d'une émulsion de savon et de pétrole, mais ce moyen est coûteux, surtout dans les régions où l'eau est rare.

Le moyen chimique le plus employé en $\mathrm{E}$ s p a g n e est le "zotal»; on en use surtout lorsqu'on se sert des barrages en zinc, car il n'attaque 
pas les bandes métalliques. On l'administre au moyen de pulvérisateurs, à divers degrés de concentration dans l'eau : à Io \% contre le "mosquito", à $25 \%$ contre la "mosca ", à $40 \%$ contre le "saltón ".

Après plusieurs expériences pratiquées dans le but de comparer de nombreux insecticides, on a choisi et on emploie, en It a li e, l'émulsion de savon et d'huile lourde de goudron, d'après la formule proposée par M. LUNARDONI. La meilleure manière de la préparer est la suivante : on fait dissoudre dans 66 litres d'eau un peu chaude $6 \mathrm{~kg}$ de savon mou (jaune) de potasse; on mélange et on continue à chauffer jusqu'à ébullition. On verse alors peu à peu dans le liquide, et toujours en mélangeant, 30 litres d'huile lourde de goudron et on laisse bouillir le tout pendant une dizaine de minutes.

Avant d'en faire usage, cette émulsion est diluée selon 1'âge des insectes: ainsi, on ajoute à chaque partie d'émulsion concentrée 5 parties d'eau pendant les deux premières semaines de vie des insectes; plus tard 4, puis 3, ensuite 2 et lorsque les insectes sont ailés et qu'on travaille le jour, on ajoute une partie et demie d'eau à chaque partie d'émulsion ; la nuit la proportion adoptée est une partie d'émulsion concentrée et une d'eau.

L'insecticide est répandu au moyen de pulvérisateurs dont nous avons dejà eu l'occasion de parler lorsque nous nous sommes occupés de la destruction de ces orthoptères, en Italie, à l'aide de la flamme de pétrole additionné de benzine. Il faut d'abord réunir les insectes à détruire, s'ils ne le sont pas déjà. Dans ce cas également, il sera opportun de munir les pulvérisateurs de tubes "cirés".

Pour ralentir l'évaporation de l'insecticide lorsqu'il est répandu sur les insectes, on y ajoute de $I$ à $2 \mathrm{~kg}$ de sel de cuisine par hl d'émulsion.

Avec I hl d'émulsion on arrose bien de 200 à $300 \mathrm{~m}^{2}$ de terrain. La même quantité d'insecticide préparé pour le premier âge des insectes revient à $2,60 \mathrm{fr}$; pour les stades suivants I hl coûte $3,20 \mathrm{fr}$; $3,80 \mathrm{fr}$; $4,30 \mathrm{fr}$ et $5,20 \mathrm{fr}$.

Du I6 avril au II juillet I9I4, on a traité avec cette émulsion, dans les communes de Bronte et de Maletto (prov. de Catane) seulement, environ 480 ha de terrain et détruit à pe1 près $400000 \mathrm{~kg}$ d'insectes jeunes et adultes. Ce moyen de lutte n'est pas seulement très efficace et remarquablement économique, mais il est aussi exempt de tout danger.

En Autriche, on a reconnu l'efficacité contre les jeunes larves d'un mélange composé de $2 \%$ de savon mou et de I $1 / 2$ à $2 \%$ d'extrait de tabac; mais ce moyen de lutte ne peut pas être appliqué en grand, car il est trop coûteux.

Les vignobles abondamment pulvérisés de bouillie bordelaise et largement saupoudrés de soufre ou mieux encore d'un mélange de chaux vive et de soufre en poudre, en parties égales, se trouvent protégés contre 1 'attaque de ces orthoptères.

On a obtenu de bons résultats en B 11 ga ri e en faisant usage de la mixture "Griloff" composée de I kg de vert de Paris dissous dans 4 litres d'ea1, de $2 \mathrm{~kg}$ de sel de cuisine et de $60 \mathrm{~kg}$ de crottin de cheval frais. 
En Grèce, les moyens chimiques employés en IgI2, comme ayant été reconnus les plus pratiques après les expériences de l'année précédente, furent les suivants.

Avant tout, le pétrole dénaturé. Les insectes aspergés de ce liquide meurent instantanément, quel que soit leur âge. Les aspersions ont lien le soir quand les insectes se trouvent rassemblés pour passer la nuit, ou mieux, le matin, avant qu'ils ne se dispersent et lorsqu'ils sont encore engourdis par le froid nocturne. On doit employer pour cela des pulvérisateurs spéciaux sans caoutchouc qui serait détérioré par le pétrole. Faute de pulvérisateurs, on peut se servir d'arrosoirs à pommes très fines, mais on risque de gaspiller le liquide, ce qui est à considérer, le pétrole dénaturé coûtant en dépôt, en Grèce, 5 fr les $30 \mathrm{~kg}$. Pendant la campagne, le Ministère de 1'Ėconomie nationale a distribué Io 000 caisses de pétrole de $30 \mathrm{~kg}$ chacune aux communes pauvres, et 500 caisses ont été vendues aux communes riches.

L'acide phénique impur 98/99 mêlé à 1'eau dans la proportion de 3-4\% a été pareillement employé. Ce produit présente plusieurs avantages: i1 est d'un transport facile, ne détériore pas les pulvérisateurs et coûte fort peu puisqu'on ne le paie pas plus de 50-60 centimes par kg. Quoiqu'il se dissolve difficilement dans 1'eau, pourtant lorsqu' on l'agite fortement, il forme un liquide laiteux qui, pulvérisé sur les insectes, les tue presque instantanément surtout dans leur jeune âge. Son seul inconvénient est d'être un peu caustique et de demander par conséquent dans son emploi certaines précautions. Le Ministère qui s'en était procuré Ioo tonnes en bidons de 25 ou de Ioo $\mathrm{kg}$ les a distribués gratuitement.

Dans les lieux où il s'est produit des invasions imprévues et qu'on n'avait pu approvisionner en temps voulu avec les insecticides précédemment cités, on a fait usage de savon ordinaire dissous dans de l'eau chaude à raison de $4-5 \%$. Celui-ci est d'une très remarquable efficacité contre les tout jeunes insectes qu'il tue par asphyxie. Il est pulvérisé abondamment sur les insectes à l'aide de pulvérisateurs ou d'arrosoirs à pommes très fines. Il présente 1'avantage de ne pas trop abîmer les plantes pulvérisées, sans compter la modicité relative de son prix et la facilité que l'on a de s'en procurer dans n'importe quel coin de la contrée.

Pour faire la guerre aux larves de Locusta migratoria, il est d'usage au $\mathrm{J}$ a $\mathrm{p}$ o $\mathrm{n}$, de creuser un trou dans le sol ou encore d'enterrer un pot dans lequel on verse du pétrole; puis on donne la chasse aux larves pour les y faire tomber.

S'il s'agit, au contraire, delutter contre l'Oxya velox, qui chaque année cause de si grands dommages aux rizières japonaises, on commence par verser de l'eau dans les semis de riz jusqu'à une profondeur d'un "sun " environ ( I "sun " = 3,0303 cm), on y verse ensuite du pétrole mélangé de poudre de pyrèthre dans la proportion d'un "shô " (I "shô" = I,8039I4 litres) pour un " $\tan »(\mathrm{I}$ " $\tan »=0,099 \mathrm{I} 7 \mathrm{ha}$ ); et 1'on frappe légèrement les feuilles des jeunes plantes avec nne canne de bambon, pour faire tomber les larves qui y sont attachées.

Pour préparer le mélange de pétrole et poudre de pyrètlıre, on jette 
20 "momme» (75g) de poudre de pyrèthre dans un "shô" de pétrole; on laisse infuser pendant 24 heures après avoir bien mélangé, et 1'on filtre à travers du coton.

On combat aussi l'O. velox de la façon suivante : après avoir versé du pétrole dans une fosse d'écoulement, on chasse les insectes pour les y faire tomber.

On se sert, en Chine, de cendre végétale, de chanx, d'huile de soya, etc. Comme cette huile coûte un peu cher, on la remplace par de l'huile de bois (I) ; cette dernière, une fois mélangée avec la cendre et la chaux, est répandue sur les cultures: les insectes ne s'arrêtent pas sur les champs ainsi protégés.

Nous avons déjà dit, à propos des Ettats Fédérés II a 1 a is, qu'on y emploie contre les jeunes insectes capturés par le moyen mécanique dont nous avons fait la description, non seulement la kérosène, mais aussi le sulfure de carbone.

On y a également coutume, dans des circonstances déterminées, de refouler les orthoptères dans des étangs, des fosses ou des tranchées dans lesquels on a préalablement versé de la kérosène. Ce système a été souvent employé lorsque des insectes étaient entrés dans une rizière; on verse alors de la kérosène dans les parties extérieures du champ de riz et des coolies y refoulent les insectes.

Mais, le moyen chimique le plus largement employé dans ces Etats et qui constitue actuellement la méthode adoptée partout oì il n'y a pas à craindre l'empoisonnement du bétail en pâture consiste dans les pulvérisations d'arsénite de soude.

La solution la plus économique et la plus efficace est préparée de la manière suivante : $2,5 \mathrm{~kg}$ d'arsénite de soude dissous dans I hl d'eatr; on fait bouillir pendant Io minutes ou un quart d'heure; puis on y ajoute $3,750 \mathrm{~kg}-5 \mathrm{~kg}$ de sucre ou de mélasse, etc., substances qui exercent une attraction sur les insectes.

Dans la pratique, pour doser les différents ingrédients, on emploie des récipients faciles à se procurer (bidons à kérosène, boîtes à cigarettes, etc.) dont la capacité correspond approximativement aux proportions de la susdite formule.

Un hectolitre du liquide ainsi obtenu coûte, sur place, environ $2 \mathrm{fr}$.

Quand les insectes sont très jeunes on fait usage d'une solution plus diluée.

Pour l'utiliser, on emploie, comme les meilleurs, des pulvérisateurs à dos d'homme, dont le jet forme un nuage très léger, ce qui rend le traitement plus efficace et plus économique.

(I) Il est fort probable que l'on fait allusion ici an "wood oil of China " extraite de deux euphorbiacées, 1'abrasin (Aleurites cordata) et le bancoulier ou noyer des Moluques ( $A$. moluccana $=A$. triloba). Il ne faut pas confondre ce produit avec la véritable huile de bois ("wood oil") que l'on tire surtout de quelques espèces de Dipterocarpus (fanille

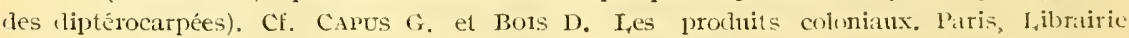
Armand Colin, I952, pp. 350-3I3, 474-477, fig. II2, I6I-I62. 
Avec un jet convenable, un pulvérisateur contenant I3,5 litres de la solution peut suffire pour $250 \mathrm{~m}^{2}$ environ, de sorte que le contenu de 40 pulvérisateurs suffit pour I ha ; dans des conditions normales, deux journées et demie de coolie peuvent traiter cette superficie. Le temps nécessaire et 1a` quantité desolution employée et, par conséquent, le coût du traitement, varient naturellement selon le genre de la végétation à arroser.

L,e traitement commence le matin de bonne heure, généralement à $6 \mathrm{~h}$ zo et on le continue avec avantage jusqu'à II h 30. Par un temps couvert ou s'il y a du brouillard, le travail peut se faire pendant toute la journée, car le soleil qui rend les insectes plus actifs fait alors défaut.

On empoisonne avec la solution la végétation que les insectes sont en train de brouter en répandant l'insecticide tout autour de la bande ou sur son front, dans la direction où elle s'avance. Il n'est pas possible, naturellement, d'établir a priori des règles définitives à ce propos ; on étudie sur place les conditions les plus convenables pour assurer au traitement la plus grande valeur pratique.

Lorsque la distribution du poison contre une génération d'insectes est terminée, on fait parcourir une seconde fois la localité traitée pour empoisonner les insectes qui auraient pu échapper à la première pulvérisation.

On'prend ensuite les précautions nécessaires pour éviter que les champs traités récemment ne soient visités par le bétail ou que l'herbe empoisonnée ne soit fauchée comme fourrage.

On a traité en tout par ce moyen, de septembre I9I3 à novembre I9I4, dans les Ettats Fédérés Malais (Selangor, Négri-Sembilan et Pahang), 2804 bandes. En I9I5, on empoisonna 5 bandes dans le Selangor et 5488 dans le Négri-Sembilan.

Cette méthode est appliquée également, depuis I9I4, dans 1'Etat de J o hore. On y a empoisonné I5I bandes du 24 novembre au 29 décembre de cette année-là. En I9I5, on traita I 068 bandes.

A Khori, dans 1'I nd e, on a reconnu que, dans la lutte contre le Schistocerca tatarica, une solution de $30 \%$ de savon ou une solution à $25 \%$ de liquide désinfectant (" sanitary fluid ») cu bien encore rne quantité égale d'une émulsion de pétrole brut, sont d'une grande efficacité. A l'aide de ces solutions, on détruit au moins $90 \%$ des insectes arrivés à leur troisième ou quatrième stade.

Dans l'île de Chy pre, on se servait autrefois de moyens mécaniques et plus spécialement, jusqu'en I883, de l'appareil qui porte précisément le nom de cypriote; depuis I894, on capturait les insectes au moyen de filets; en IgII, les zones infestées furent arrosées avec des solutions d'arsénite de soude, comme cela se pratique dans 1'Afrique du Sud. Les résultats ont été si satisfaisants qu'on a proposé de répandre l'usage de cet insecticide.

En A 1 gé rie, on a reconnn comme étant très efficace pour la destruction des criquets l'épandage de solutions crésylées préparées à la dose de 8 à ro litres de crésyl par roo litres de solution. Au moyent d'un pulvérisateur, 
un homme peut répandre par jour 300 litres de solution sur un demi-hectare. Dans certains cas, seront utiles aussi les appareils à bât.

En Tunisie, on emploie les pulvérisations au crésyl - dans la proportion de Io à I5 \%, selon qu'il s'agit de criquets éclos peu de jours avant ou d'insectes nés depuis une huitaine de jours - au - pétrole — à raison de 8 à $20 \%$ toujours suivant le développement atteint par les criquets - et on y emploie aussi les pulvérisations au savon anglais ou au savon du pays, à $7 \%$. Ces dernières sont économiques et leur usage n'entraîne pas les inconvénients et les dangers qui accompagnent les deux premiers moyens susmentionnés.

En Erythrée on fait usage avec succès contre les jeunes insectes de pulvérisations de solutions savonneuses à $6 \%$.

Lorsqu'on constata 1'apparition de ces orthoptères au Congo belge, la méthode chimique employée pour les combattre consista, d'après l'exemple de 1'Afrique du Sud, dans 1'application d'arsénite de soude. Les formules y relatives, d'après la circulaire, déjà mentionnée, du Gouverneur général en date du 4 juin I907, sont les suivantes:

a) contre les insectes très jeunes :

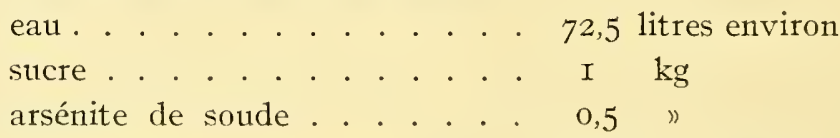

b) contre les insectes arrivés à la moitié de leur développement:

eaut

54,5 litres environ

stucre

$0,75^{7 \mathrm{~kg}}$

arsénite de soude

0,5 »

c) contre les insectes ayant presque atteint leur complet développement mais encore dépourvus d'ailes, et même contre les ailés:

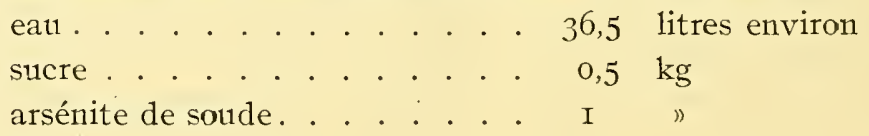

Dans l'Afrique orientale allemande, vu les résultats pratiques obtenus dans 1'Afrique du Sud, c'est aussi l'arsénite de soude qui a été choisi comme moyen de lutte. Mais on y a aussi obtenu d'excellents résultats, dans la destruction des jeunes insectes, avec une solution de savon à $3 \%$ environ, à l'occasion de la dernière apparition que ces orthoptères firent dans la Colonie. Et même, pour le moment, il n'y a de renseignements que sur l'efficacité de la solution savonneuse.

Le Gouvernement de la Province de Mozambique emploie actuellement une mixture appelée "Locusticide", mise en vente depuis plusieurs années, dans des récipients de I "gallon" (4,54 litres). C'est une solution concentrée d'arsénite de soude et de sucre dans des proportions déterminées; il 11'y a' quı'à la diluer avant de s'en servir. L'avan- 
tage de ce produit vis-à-vis de l'ancienne préparation in loco de la solution d'arsénite de soude et de mélasse, employée dans les Colonies britanniques de l'Afrique du St1d, consiste dans la réduction de son volume - lorsqu'on l'utilise par grandes quantités - et, de plus, dans celle des frais énormes et des difficultés relatives à son transport.

En voici les différentes formules:

a) contre les insectes très jeunes:

$\begin{array}{rrr}\text { "Locusticide " . . . . . . . . . . . . . } & \text { I litre } \\ \text { eau . . . . . . . . . . . . . . . } & 54 \text { litres }\end{array}$

b) contre les insectes d'un développement moyen :
"Locusticide » . . . . . . . . .
I litre
eat1
43 litres

c) contre les insectes presque complètement développés, mais encore dépourvus d'ailes:
"Locusticide " . . . . . . . . .
I litre
eau.
32 litres

L'application du poison se fait au moyen de pulvérisateurs à seau.

Lorsqu'une invasion d'insectes encore non ailés se produit dans les cultures on traite légèrement, avec cette préparation, de manière à ne pas trop l'humecter, l'herbe qui se trouve aux alentours de la propriété, et on y refoule les insectes.

Dans les plantations de canne à sucre du Zambèze, la méthode des pulvérisations est appliquée, selon les circonstances, en plus du ramassage des œufs dont nous avons parlé précédemment. Mais on a constaté que l'insecticide endommage quelquefois les rejetons tendres des cannes.

Profitant de l'expérience acquise par les plus anciennes Colonies de 1'A. frique du Sud, la Rhodésia méridionale emploie surtout les solutions arsénicales sucrées pour la destruction des jeunes insectes. Les formules en sont les mêmes que celles recommandées dans les Colonies méridionales de 1'Afrique, à savoir :

a) pour insectes très jeunes, dans les 2 premières semaines de leur existence:

arsénite de soude. . . I " pound" $(0,453 \mathrm{~kg})$

sucre ou mélasse . . . . 2-4 "pounds " (0,900-I,800 kg environ)

eau . . . . . . . I6 "gallons" $(72,5$ litres environ)

b) pour insectes d'un développement moyen, de 2 à 5 semaines: arsénite de soude... . I " pound"

sucre ou mélasse .. . 2-4 " pounds"

eat1........ I " gallons " $(54,5$ litres environ) 
c) pour insectes plus grands avec les rudiments des ailes bien définis, de 5 à 8 semaines :

arsénite de soude

I " pound"

sucre ou mélasse . . . 2-4 "pounds"

eau

8 " gallons " $(36,5$ litres environ $)$.

On se sert généralement de ce poison en le pulvérisant sur l'herbe devant la bande en marche. On a largement employé à cet usage le pulvérisateur à seau type "Deming's Success", pourvu d'un jet type Bordeaux.

I1 est quelquefois plus facile d'empoisonner avec cette solution une certaine quantité d'herbe verte ou de fourrage et de la répandre devant les insectes en marche.

Les jemnes orthoptères mangent l'herbe empoisonnée, meurent et sont dévorés par ceux qui suivent et qui meurent à leur tour. L'usage du poison en question est très efficace.

Dans le Sud-Ouest africa in a 11 emand, la méthode choisie est également de pulvériser sur les jeunes insectes une solution d'arsénite de soude. Mais, comme depuis I907, époque où la lutte a été organisée, ces orthoptères n'ont pas pris dans le territoire en question une importance digne de remarque, il n'y a pas eu de résultats pratiques relativement à l'efficacité de l'insecticide adopté.

Enfin, la solution d'arsénite de soude est employée dans l'Un ion de 1'Afrique du Sud comme le moyen de lutte généralement préféré contre les insectes non ailés que l'on connaît dans cette Colonie, ainsi que dans les régions voisines, sous le nom vulgaire de "voetgangers ". Pour être plus précis, nous dirons que c'est après les résultats excellents que cet insecticide a donnés au Natal, qu'il a été si largement adopté dans les autres régions.

Nous avons donné, à propos de la Rhodésia méridionale, les trois formules qui, sur la proposition du Conité de contrôle du "South African Centra1 Locust Bureau "), furent appliquées à l'origine, selon le stade d'évotion des orthoptères à détruire.

Au début, la quantité de mixture vénéneuse nécessaire à la destruction des insectes était préparée au fur et à mesure des besoins du moment.

On reconnut ensuite qu'il était plus pratique de se servir d'une solution très concentrée, déjà prête à être employée, après y avoir ajouté la quantité d'eau voulue.

Ce fut ainsi qu'en Igo8, le Natal fournit les fonctionnaires chargés de la lutte et d'autres personnes encore de la mixture d'arsénite mélangé de mélasse, préparée par les "South African Sugar Refineries, Ltd." de South Coast Junction (Natal) ; elle était placée dans des caisses contenant chacune quatre bidons à fermeture hermétique, de la capacité de I, 5 "gallons ) (6,8I litres) ; chaque caisse était considérée comme suffisante pour 3 r6 "gallons.) (I $4,33 \mathrm{hl}$ ) d'eat.

Plus tard (Igog-Io), le Convernement du Cáp a tronvé qu'il était plus pratique et plus économique de faire préparer lui-même à Rose- 
bank, près de Cape 'Town, le mélange prêt à être employé; le liquide est contenu dans des bidons de 6,8I litres, portant en grandes lettres l'inscription: "C. G. H. Locust Poison. Vergif.". Chaque bidon contient 5 " pounds " $(2,265 \mathrm{~kg})$ d'arsénite de soude à $69 \%$ d'arsenic blanc, I "gallon " (4,54 litres) de mélasse ou environ ro "pounds " $(4,530 \mathrm{~kg})$ de sucre brun et une petite quantité d'eau.

Pour préparer le poison, on fait dissoudre 200 "pounds" (90,7I kg) d'arsénite de soude dans I5 "gallons " environ (68, Io litres) d'eau bouillante et on y ajoute de l'eau jusqu' à ce que le tout ait atteint 20 "gallons " (90,8 litres) ; on verse ensuite dans chaque bidon un demi "gallon ) $(2,27$ litres) de cette solution, on y ajoute I "gallon " de mélasse et le tout est soigneusement remué. Cela constituait le "Locust Poison" toujours prêt pour l'usage avec addition d'eau.

Chaque bidon, tous frais compris, coûtait environ 3,80 fr à Rosebank.

La dilution conseillée était de I partie de la mixture dans 66 d'eau pour les "voetgangers" éclos depuis peu et de I partie dans 50 pour les insectes comptant une quinzaine de jours de vie.

La solution est pulvérisée sur l'herbe, les buissons, etc. où les insectes sont occupés à brouter; quand ces orthoptères sont encore petits, on peut diriger le jet entre eux et autour d'eux ; lorsqu'ils sont grands et en mouvement, il vaut mieux répandre l'insecticide sur une bande d'herbe le long du front de la bande des insectes.

Quant au pulvérisateur, on a d'abord choisi un appareil du type "Deming's Success ", avec jet type Bordeaux, comme pour la Rhodésia méridionale. Plus tard, le pulvérisateur "Crescent», de fabrication anglaise, a été trouvé tout aussi bon que le premier.

Le moment le plus opportun pour faire les pulvérisations, est celui où les insectes se sont rassemblés pour passer la ntit, ou le matin de bonne heure, avant qu'ils ne se mettent en mouvement. Il faut éviter de trop humecter l'herbe traitée par l'insecticide.

Eitant donné la nature de ce dernier, il est nécessaire de prendre des précautions simples et faciles afin d'éviter les irritations de la peau causées par la causticité du liquide et aussi pour prévenir les cas d'empoisonnement du bétail en pâture.

Selon le caractère du "veld", on a reconnu parfois qu'il était plus pratique d'employer les appâts empoisonnés au lien des pulvérisations; nous avons déjà mentionné ce moyen en parlant de la Rhodésia méridionale. On se sert généralement de fourrage vert haché ou d'un autre appât que l'on trempe dans la solution d'arsénite de soude dulcifiée et que l'on répand sur le sol devant la bande des insectes, qui s'en repaîssent et s'empoisonnent. Dans les zones plus arides du territoire de 1'Union, cette méthode a été trouvée plus avantageuse, tandis que dans les districts cù l'herbe est assez abondante, les pulvérisations paraissent práférables.

La mixture Criddle ("Criddle mixture»), qui porte le nom de son inventent, M. Norman CRIDDL, E, a été recomnue, au Ca 11 a da, comne le poison le plus efficace. I'iclée en est venue de l'attrait que le fumier 
de cheval exerce sur ces insectes. On mélange très soigneusement dans un baril 60 "pounds" ( $27 \mathrm{~kg}$ environ) de crottin avec I "pound") $(0,453 \mathrm{~kg})$ de vert de Paris et 2 "pounds " $(0,906 \mathrm{~kg})$ de sel dissous dans un demi seau d'eau. Après que le tout a été bien mélangé, on transporte le baril sur un char dans la localité infestée ou suspecte et sans qu'il soit nécessaire de le descendre à terre, on répand la mixture sur le sol, au moyen d'une bêche ou d'une truelle. La meilleure manière d'employer ce mélange, c'est d'en verser tous les jours une petite quantité sur le terrain infesté plutôt que d'en répandre une grande quantité à des intervalles plus longs.

Aux Etats-Unis, les deux moyens chimiques dont on use le plus sont la mixture Criddle, que nous venons de faire connaitre, et la mixture ou pâte de son empoisonnée ("poison bran mixture" ou "poisoned bran mash »).

La première se prépare en mélangeant un demi baril de fumier de cheval frais avec I " pound " $(0,453 \mathrm{~kg})$ de sel et I de vert de Paris; si le fumier n'est pas frais, le sel doit être dissous dans l'eau et mélangé ensuite avec le fumier et le poison.

I a seconde se compose d'un mélange de $0,453 \mathrm{~kg}$ de vert de Paris avec 25 "pounds" (II,325 kg) de son de blé; on donne au tout un certain degré de consistance à l'aide d'eau sucrée. Cet appât contenant en plus le jus, la pulpe et l'écorce de certains fruits (oranges ou citrons) a donné parfois de bons résultats.

I a mixture Criddle a été appliquée généralement avec grand succès, avant que les insectes ne fussent pourvus de leurs ailes.

Une solution composée d'arsenic blanc en poudre ( $\mathrm{I} \mathrm{kg}$ ), de soude caustique $\left(25^{\circ} \mathrm{g}\right)$ et d'eau ( $\left.\mathrm{h} h \mathrm{~h}\right)$, s'est montrée très efficace à Co s t a $\mathrm{R}$ i c a contre les jeunes insectes en la répandant sur l'herbe basse; on la fait bouillir pendant ro minutes, et on y ajoute du sucre pour en favoriser l'adhérence et attirer les insectes à détruire.

A Porto-Rico, les appâts empoisonnés employés pour combattre un autre orthoptère nuisible, le Scapteriscus didactylus Latr., connu sous le nom vulgaire de "changa ", tuent aussi un certain nombre d'insectes de l'espèce Schistocerca columbina.

Dans l'île de la Trinité, durant une apparition de sauterelles dans une plantation de canne à sucre, on a obtenu un excellent succès en saupoudrant les plantes de vert de Paris.

Les sauterelles qui apparurent en I9I5 dans l'île de Patos, située dans le voisinage de la Trinité, furent efficacement combattues, par les soins du Gouvernement de cette dernière île, au moyen de la pâte de son empoisonnée ("bran mash»), préparée selon la formule de HuNTER et CraAsSEN, sauf que les oranges ou les citrons furent supprimés de la pâte. I1 faut préparer le mélange le jour même ò̀ il doit être employé. On mêle d'abord à sec 2, 5 " pounds " (un peu plus de I kg) de vert de Paris on d'arsenic blanc et 50 "pounds " $(22,5 \mathrm{~kg}$ environ) de son; puis on fait dissoudre à part 4 "quarts" (5, 5 litres environ) de mélasse dans 5 "gallons " (22,75 
litres) d'eau; enfin on mélange le son empoisonné avec le susdit liquide et l'on y ajoute une quantité d'eau suffisante pour former une pâte humide.

A Patos, on a répandu cette pâte, à la volée, dans la proportion de 8 " pounds" (3,500 $\mathrm{kg}$ environ) par " acre» $(0,405 \mathrm{ha})$.

Au B r és i 1, on emploie, au moyen de pulvérisateurs, l'arsénite de soude, le vert de Paris mélangé à du sucre, l'insecticide Werneck à 5\% et $1^{\prime}$ " acaroina " à $20 \%$.

L'arsénite de soude et l'insecticide Werneck ont donné les meilleurs résultats.

En Argentine, on utilise pour la destruction de la "mosquita» (insecte très jeune) et de la "saltona " (dont le développement est un peu plus avancé), depuis leur naissance jusqu'à leur première mue, les arrosages directs contre les bandes avec des liquides insecticides dilués; les plus généralement employés sont des dérivés du phénol ("acaroina ", "acridina ", créoline, etc.). On se sert aussi de solutions savonneuses. Ces procédés ont d'autant plus d'efficacité, qu'ils sont appliqués dans les stades les moins avancés de la vie de la "langosta ".

Au Paraguay, on a employé avec succès les solutions savonnenses. Aux époques de sècheresse ou de pluies rares, on a obtenu de bons résultats en empoisonnant la végétation dans le voisinage des insectes, surtout au moyen de solutions arsénicales mélangées avec du sucre.

Dans 1'Uruguay, on emploie l'" acridina », 1' "acaroina », la créoline, la nicotine, le savon de potasse, la kérosène et en général tout liquide insecticide d'un usage courant. L'application en est faite au moyen de pu1vérisateurs ou avec des arrosoirs; la deuxième méthode est la plus usitée.

En Australie, on a employé avec succès au Queensiand plusieurs préparations chimiques, bien que, étant donnée l'étendue du front d'invasion des insectes, les applications n'aient pu être faites que d'une manière assez restreinte.

I es insecticides généralement employés sont 1a solution d’arsénite de soude et la solution de savon.

Pour la première, on faisait dissoudre Io "pounds" ( $4,5 \mathrm{~kg}$ environ) d'arsénite de soude dans I "gallon " (4,54 litres) d'eau chande et on y ajoutait 2 fois son volume de mélasse. Pour pulvériser les très jeunes insectes, on a mélangé I partie de cette solution avec 46 parties de son volume d'eau; pour les grands insectes, I partie de la dite solution et 23 parties de son volume d'eau.

La préparation de la solution de savon s'obtient en faisant bouillir du savon dur ("Sunlight ), dans la proportion de I "pound " $(0,453 \mathrm{~kg}$ ) dans 5 "gallons" (23 litres environ) d'eau.

On a reconnu que cette solution était très efficace.

Dans la Nouvelle-Cralles du Sud, on a proposé ou expérimenté à plusieurs reprises, surtout contre les jeunes insectes, différentes préparations chimiques: la pâte de son empoisonnée, les pulvérisations avec un 
mélange à base d'arsenic, de soude et de mélasse, avec des émulsions de kérosène ou de savon ou de "Little's Dip" (liquide phéniqué pour laver les moutons), avec une suspension de kérosène et d'eau, avec une solution d'arsénite de soude, etc.

Il a été prouvé finalement que les pulvérisations d'arsénite de soude, d'après la méthode appliquée à l'origine dans l'Afrique du Sud, peuvent limiter le fléau.

Dans l'Etat de Victoria, lorsqu'il s'agit de sauver des plantes importantes, on considère comme très efficace une faible émulsion de kérosène (I dans 30), ou bien un mélange composé de I "pound» $(0,453 \mathrm{~kg})$ de vert de Paris, 6 "pounds " $(2,75 \mathrm{~kg})$ de chaux et 6 "pounds 》 de mélasse dans I60 à I80 "gallons " (726-8I7 litres environ) d'eau. 


\section{UTILITÉ D'UNE ENTENTE INTERNATIONALE POUR LA LUT'TE CONTRE LES SAUTERELILES}

Nous avons essayé d'exposer dans les pages précédentes - autant du moins que les documents dont nous avons pu disposer nous l'ont permis - l'état actuel de la question des sauterelles dans les divers pays.

Nous avons parlé successivement de l'historique et de la distribution géographique de ces insectes sur la surface de la terre; nous avons indiqué quelles sont les espèces que l'on considère comme nuisibles à l'agriculture mondiale, et nous avons exposé toutes les particularités biologiques qu'il nous a été possible de recueillir concernant plusieurs d'entre elles; nous avons parlé, enfin, de l'organisation en vigueur relative à la lutte contre les sauterelles ainsi que des moyens en usage pour les combattre dans les régions les plus diverses du globe.

L'examen de toutes les données, consignées tour à tour dans les divers chapitres, permet de conclure que, si, d'une part l'état encore incomplet et incertain des connaissances acquises sur la biologie des sauterelles; si, d'autre part, la forme imprévue, terrifiante et récurrente affectée dans la plupart des cas par les manifestations du fléau; si l'étendue, parfois énorme, de la zone atteinte et la nature même des dommages causés par ces orthoptères ne permettent pas de les vaincre d'une manière immédiate, complète et durable, il n'en est pas moins vrai que l'intervention de l'homme peut, dans des limites notables, réduire la fréquence et l'importance des pertes que les sauterelles sont à même d'occasionner.

Il est bon ici d'établir encore une fois la distinction entre les espèces ayant un caractère presque stationnaire ou se déplaçant dans des limites de territoire relativement restreintes et qui de ce fait peuvent être comprises sous la dénomination de petits migrateurs, et celles qui, étant douées de la faculté de parcourir des distances très grandes, peuvent être considérées comme de grands migrateurs.

En ce qui concerne les espèces de la première catégorie, il est évident que la lutte sera d'autant plus féconde en bons résultats qu'elle se basera principalement sur des mesures préventives rationnelles, destinées à être appliquées dans les pays mêmes où ces insectes se développent et causent des ravages.

Mais ce sont les grands migrateurs qui attirent le plus, et à juste titre, l'attention d'un grand nombre de pays du globe sujets à leurs invasions 
périodiques et désastreuses. Or le moyen le plus efficace, le seul qui puisse, dans ce cas, permettre aux pays intéressés de lutter avec grand espoir de succès contre ces ravageurs de leurs cultures, se trouve, sans contredit, dans la réunion et le concours de toutes les forces isolées en vue de la défense commune, systématique et permanente; ce qui, en d'autres termes, équivaut à l'adoption de mesures internationales appropriées.

Personne n'ignore que le principe de l'opportunité, de la nécessité même, d'accords internationaux pour la lutte contre les fléaux de l'agriculture, a été depuis longtemps compris et chaudement appuyé et qu'il a été admis et reconnu officiellement. Citons comme plus ancien exemple 1a Convention phylloxérique signée à Berne en I878, revisée en I88I; ensuite la Ccnvention internationale pour la protection des oiseaux utiles à l'agriculture, signée à Paris en I902, et plus récemment encore, la Convention phytopathologique qui fut conclue à Rome en I9I4 et qui couronna les travaux de la Conférence internationale de Phytopathologie, convoquée, sous les auspices de l'Institut international d'Agricnlture, par le Gouvernement de la République française, d'accord avec le Gouvernement du Royaume d'Italie.

A part ces ententes, dont la première avait pour but la défense contre 1'aphidé bien connu de la vigne et les deux autres, directement ou indirectement, la lutte contre les fléaux des cultures en général, nous savons, d'après ce qui a été dit précédemment, que les sauterelles elles-mêmes ont déjà été l'objet de mesures internationales particulières, mais se bornant à certaines régions du globe.

Nous entendons parler en premier lieu de l'institution temporaire du "South African Central Locust Bureau " établi à Prétoria, avec le concours des possessions de la Grande-Bretagne, de 1'Allemagne et du Portugal les plus directement intéressées à la question, et, en second lieu, de la signature de la Convention relative à la recherche des foyers d'origine du Schistocerca paranensis, conclue entre les pays sud-américains ravagés par cette sauterelle, ayant adhéré à la Conférence internationale de "Defensa Agrícola » de Montevideo. On sait que cette Conférence amena aussi la stipulation - et cette fois avec l'adhésion d'un plus grand nombre d'Etats sud-américains - de deux autres Conventions concernant l'une les fléaux de l'agriculture en général et l'autre les fléaux inconnus dans les territoires des Etats signataires.

Les résultats pratiques, obtenus par le "South African Central Locust Bureau " pendant les quatre ans qu'il vécut, furent tellement satisfaisants, que l'on a éprouvé le désir, d'ailleurs réalisé, qu'après la suppression du Bureau, l'organisation qui en dépendait continuât à exister et à donner ses fruits par l'intermédiaire de la Division d'Entomologie annexée an Département de l'Agriculture de 1'Union de l'Afrique du Sud.

Cela posé, il est encourageant de pouvoir noter le fait que le principe d'une coopération internationale pour la lutte contre les sauterelles a fait son chemin et qu'il est désormais apprécié à sa juste valeur même par des pays qui n'ont pas pris part auparavant aux accords que nous renons de mentionner. Fin effet, d'après ce qui résulte de l'enquête ouverte par 
l'Institut international d'Agriculture, lorsqu'il s'est agi de recueillir les données nécessaires à l'élaboration de ce Rapport, plusieurs pays menacés périodiquement et gravement, dans les mêmes conditions que les Colonies de l'Afrique du Sud et les Etats de l'Amérique méridionale, se sont prononcés en faveur d'une entente intetnationale à ce sujet. Nous ajouterons, en nous basant encore sur les réponses parvenues à l'Institut, que cette entente, si désirable et si avantageuse pour les pays éprouvés par les vraies sauterelles migratrices, est considérée aussi indirectement utile aux Etats dont les. territoires donnent 1'hospitalité à des représentants des sauterelles compris parmi les petits migrateurs; car, indépendamment des mesures à prendre sur place selon les besoins particuliers, ces pays pourraient évidemment bénéficier de l'expérience acquise dans la lutte par ceux qui seraient groupés et unis en vertu d'un accord international.

Les pay's qui, d'une manière ou de l'autre, se sont déclarés favorables en principe à une entente internationale sont, d'après l'ordre géographique suivi jusqu'ici: le Portuga 1, l'Espagne, l'Italie, l'Autriche, la Hongrie, la Roumanie, la Grèce, la Chine, l'Inde, le Maroc, la Tunisie, le Cameroun, le Canada, les EtatsUnis, le Mexique, la Trinité. 



\section{INDEX BIBLIOGRAPHIQUE}

La littérature concernant les sauterelles, et, parmi celles-ci, surtout les espèces ayant un caractère migrateur bien net, est extrêmement vaste. Elle se trouve disséminée dans les publications les plus diverses du monde entier, et qui ne sont pas toujours facilement accessibles, aussi serait-il malaisé de citer les nombreux auteurs qui ont écrit sur ces orthoptèrès, sans craindre d'enl omettre et même des meilleurs.

D'après ce qu'affirme J. KÜNCKEL D'HERCULAIS, on comptait déjà, en I888, plus de 500 mémoires ou notes sur le sujet qui nous intéresse. Les plus importants de ces travaux ont été signalés ou résumés dans les grands ouvrages qui parurent depuis, entre autres, dans celui, bien connu, qu'écrivit sur les invasions des sauterelles en Algérie le savant français que nous venons de citer.

La bibliographie que nous publions ici contribue à enrichir et à mettre, autant que faire se peut, au courant la documentation déjà réunie dans les travaux qui ont précédé la présente étude sur ces ravageurs des cultures, au moins pour ce qui concerne les pays dont on a parlé. Elle comprend plus de 500 titres dont le plus grand nombre peuvent être considérés comme méthodiquement groupés pour la première fois. Ils proviennent de publications de tout genre, et même toutes récentes, arrivées, la plupart, à la Bibliothèque de l'Institut international d'Agriculture et dont on a largement profité au cours des recherches qu'on vient d'exposer. Nous avons fait suivre d'une astérisque les travaux contenant une bibliographie d'une certaine étendue.

ACCARDr, S. Lettera al prefetto di Girgenti sull'invasione delle cavallette. L'Agricoltore Agrigentino, Girgenti, I 909, pp. 84-87.

ACCARDI, S. Breve relazione sulla lotta contro gli ortotteri nei territori di Racalmuto e Grotte. L'A gricoltore Agrigentino, Girgenti, r909, pp. 87-99.

ACCARDI, S. I,e cavallette a Racalmuto e Grotte. L'Agricoltore Agrigentino, Girgenti, rgro, pp. 34-35.

ACCARDI, S. Esame critico dei mezzi di lotta consigliati per la distruzione delle carallette. L'A gricoltore Agrigentino, Girgenti, I910, pp. 65-72,8I-97.

ACCARd, S. Preparianoci alla lotta contro le cavallette in Sicilia. Girgenti, Stamperia Montes, IgII, 5 pp., 3 fig.

ALdrovand, U. De animalibus insect is. Bononiae, apud Ioan. Bapt. Bellagambam, r6o2, pp. 403447 , fig.

Alfaro, A. La invasion de langosta. Revista de Educación, San José de Costa Rica, rgi 5, pp. I-7 du tirage à part, I fig. ; Centro-América, Guatemala, I9I5, vol. VII, ño. 4, pp. 636-640. 
Allard, H. A. Some Northern Georgia Acridiidae. The Canadian Entomologist, London, I9I6, vol. XLVIII, no. 8, pp. 2ヶ4-279.

Alvarez, T. Ia langosta peregrina (Acridium peregrinum nostras). Caracteres y costumbres. Historia de la invasión de I896. Dedicado á la Comisión central de extinción de la langosta por - -. Montevideo, Imprenta "Rural ", I898, 29 pp., 6 fig.

ANDreuccr, O. Delle cavallette e del modo di distruggerle. Rivista di Agricoltura, Industria $c$ Commercio, Firenze, I870, disp. VIII, IX, X, Pp. I26-I33, I9I-I96, 264-283.

AxDrews, E. A. On insects. Indian Tea Association. Scientific Department. Quarterly Joumal, Calcutta, I9I3, part 2 , pp. 33-4z.

Axdrews, E. A. A swarm of locust in the Darjeeling and Terai districts. Indian Tea Association. Scicntific Department. Quarterly Journal, Calcutta, I9I 5, part 3, pp. 63-67.

Ascírute y Fernández, C. Insectos y criptógamas que invaden los cultiros en España. IIadrid, Tipolitografia de I. Péant e hijos, I 893, passim.

Baldiccr, A. Le cavallette. Società degli A gricoltori italiani. Bollettino quindicinale, Roma, I 9 I I, vol. XVI, p1. III-II3.

B.ALL, E. D. Estimating the number of grasshoppers. Journal of Economic Entomology; Concord, N. H., I9I5, vol. 8, no. 6, pp. 525-527.

BALL, E. D. How to control the grasshoppers. Experiment Station of the Agricultural College of Utah. Bulletin I 38, Logan, Utah, I9r5, Pp. 79-I I6, I6 fig., 6 pl.

Ballou, H. A. Insect pests of the Lesser Antilles. Imperial Department of Agriculture for the West Indies. Pamphlet Series, No. 7I, Bridgetown, Barbados, I912, pp. 73-74, fig. 8o.

Barks, N. A list of works of North American entomology. U. S. Department of Agriculture. Bureau of Entomology. Bulletin no. 81, Washington, I9ro, pp. 90-97.

BAxó, E. Reseña sobre el uso de la "cerca de Chypre " y las nuevas máquinas para exterminar la langosta. Comisión de Parasitologia Agricola. Circular núm. 56, México, I90\%, 9 pp., II lám.

B.ARSACQ, J. La lutte contre les criquets. Revuc de Triticulture, Paris, I9I3, $20^{e}$ an., 1 . XXXIX, $n^{0}$ IoI 8 , pp. 852-857, fig. I 37-I $42 ; t$. XI, $n^{\circ}$ I O20, pp. II-55; $\mathbf{n}^{\circ}$ IO2I, pp. 43-50.

BÉGUET, M. Essai de destruction du Stauronotus maroccanus Thun., en Algérie, au moyell du Coccobacillus acridiorum d'Hérelle. Bulletin de la Société de Pathologie exotique, Paris, I9I4, t. VII, nos 8-9, pp. 65I-653.

BÉGUET, M. Deuxiènte campagne contre les sauterelles (Stauronotus maroccanus Thnn.) en Algérie, an moyen du "Coccobacillus acridiorum" d'Hérelle. Anmales de l'Institut Pasteur, Paris, I9I5, t. XXIX, no Io, pp. 520-536.

BÉGuEt, M. Campagne d'expérimentation de la méthode biologique contre les Schistocerca peregrina en Algérie, de déceubre I9I 1 à juillet I9I5, et en particulier dans la région de Barika (département de Constautine). Annales de l'Institut Pasteur, Paris, I9I6, t. XXX, $11^{\circ} 5$, pp. $225^{-242}$, I fig.

Béguet, M., Musso, I. et Sergent, ET. Troisième campagne contre les Acridiens ("Schistocerca peregrina " O1.) en Algérie au moyen du "Coccobacillus acridiorum "Hérelle. Bulletin de la Société de Pathologie exotique, Paris, I9I 5, t. VIII, n ${ }^{\circ}$, pp. 634-637.

BERG, C. Sobre los enemigos pequeños de la langosta peregrina Schistocerca paranensis (Burmu.). Comunicaciones del Museo Nacional de Historia Natural de Buenos Aires, Bnenos Aires, I 898 , tomo I, 1pp. 25-30.

Berlese, Ant. Cenni sulle cavallette, che in Italia danneggiano le campagne e notizie sull'iuvasione verificatasi in Provincia di Firenze (Brozzi) nell'estate del I S93. Rivista di Patologia vegetale, Avellino, I 893-I894, vol. II, 11. I0-I2, pp. 273-320, 36 fig., 3 tav.

BERLESE, ANT. Istruzione sulle cavallette nocive alle campagne e sul nodo di combatterle. Bollettino ufficiale del Ministero d'Agricoltura, Roma, I902, anno I, vol. II, p. 2 I 84 .

Bernardez, M. A defeza contra o gafanhoto. A maldição pharaonica. Chacaras e Quintacs, S. Paulo, Brazil, I9I I, vol. III, 11. 5, pp. $26-28$.

Bertoni, M. S. Informe de los trabajos de la Conferencia internacional de la Defensa Agricola 
(Montevideo, mayo I913). Boletin núm. 2, Asunción, Talleres Gráfico del Iistado, IoI3, pp. 4-9, I6-I8.

Bochiccho, N. Lot ta contro le cavallette in provincia di Messina. $R$. Scuola pratica di Agricoltura Pietro Cuppari in Messina (S. Placido Calonerò). Relazione del Dirctiore Prof. - - per l'anno scolastico r9og-rgio. Messina, Tip. D'Angelo, I9 I , pp. 39-52.

Bolle, C. Die Bekämpfung der Ameisen- und Heuschreckenplage in Südamerika. Der Tropenpflanzen, Berlin, I907, I I. Jahrg., Nr. 6, S. 392-40r.

Bondar, G. Vespas caçadoras de gafanhotos. Secretaria da Agricultura, Commercio e Obras Públicas do Estado de São Paulo. Boletim de Agricultura, São Paulo, I9r 5, I6 serie, nº. 5, pp. $442-444$, I fig.

Boy, C. Instrucções praticas para destruição dos gafanhôtos. Secretaria da. Agricultura, Commercio e Obras Públicas do Estado de S. Paulo, S. Paulo, Duprat e Comp., I 9Io, 36 pp., 50 fig.

Bor, C. A praga dos gafanhotos. Actualidade da questão, no seu duplo aspecto nacional e internacional. Relatorio do Ministro da Agricultura, Industria e Commercio, I9Iz, Rio de Janeiro, Imprensa Nacional, I9I4, vol. II, pp. II9-I28.

BREHM, A. E. Merveilles de la Nature. Les insectes, les myriopodes, les arachnides et les c1usstacées. Edition française par J. KüNckel D'HerculaIs. Paris, J.-B. Baillière et fils, I 882 (?), t. I (VII de la collection), pp. 4I0-436, 440-443, fig. 6I 7-620, 634-638, 2 pl.

Brethes, J. Sarcophaga Caridei. Una nuera mosca langosticida. Anales del Museo Nacional de Historia Natural de Bucnos Aives, Buenos Aires, I906, serie III, tomo VI, p. 297 .

BRÈtHes, J. Sobre la Brachycoma Acridiorum (Weyenb.) (Nemoraea acridiorum Weyenb.). Anales del Museo Nacional de Historia Natural de Buenos Aires, Buenos Aires, I9I2, tomo XXII (serie III, tomo XV), pp. 44I-446, fig. I-3.

Brongntart, CH. Note sur les champignons parasites du criquet pèlerin. Bullctin de la Société entomologique de France, Paris, I 892 , p. LIX.

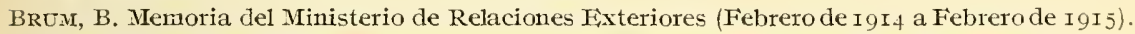
Montevideo, Imp. "El Siglo Ilustrado ", de Gregorio V. Mariño, I 9 I 5, pp. 400-422.

Bruner, I. Locusts or grasshoppers. Agricultural Experiment Station of Nebraska. Bulletin jo, Lincoln, Nebr., I90I, vol. XIII, art. T, pp. 43-54, fig. I-I 3 .

Brutrinr, A. I lavori del Consiglio dell'Agricoltura. Società degli Agricoltori italiani. Bollettino quindicinale, Roma, I9II, vol. XVI, pp. I46-I47.

BRyANT, H. C. Birds in relation to a grasshopper outbreak in California. University of California Publications in Zoology, Berkeley, I9I2, vol. I I, no. I, pp. I-20.

BRrant, H. C. A determination of the economic status of the western meadowlark (Stumella neglecta) in California. University of California Publications in Zoology, Berkeley, I9I4, vol. II, no. I4, pp. 429-430*.

BRYDEN, H. A. Locusts. The Field, London, I9I3, vol. CXXI, no. 3I34, pp. I39-I40.

ButLer, E. J. and LEFroy, H. M. Report on trials of the South African locust fungus in India. Agricultural Research Institute, Pusa. Bulletin no. 5, Calcutta, I907, 5 pp.

Caesar, I. Insects of the season in Ontario. Fonty-Fifth Amual Report of the Entomological Society of Ontario, I9I 4. Toronto, I9I5, p. 46.

CANavarr, I. Gl'insetti della vite descritti ed illustrati. Pisa, F. Spoerri, I9I2, pp. 58-6I, tar. I, fig. $I-7^{*}$.

Cape of Good Hope. Department of Agriculture. Report of the Government entomologist for the year I900. Cape Town, I 90I, pp. 54-55.

Cape of Good Hope. Department of Agriculture. Report of the Govenment entomologist for the half-year ended jume 3oth, I904. Cape Town, I905, pp. 7-9.

Cape of Good Hope. Department of AGriculture. Report of the Govemment entomologist for the half-year ended 3 Ist december, I904. Cape Town, I906, pp. I I-I 2.

Carle, G. La lutte contre les sauterelles daus les pays de l'Afrique du Sud. Colonie de Madagascar et Dépendances. Bulletin économique, Tananarive, IgI2, $\mathrm{I}_{2} \mathrm{e}$ an., ${ }_{2}^{\mathrm{e}}$ sem., no 2, pp. I63I 66 . 
Cateriano, J. G. Ira plaga de langosta en Argelia. Boletin de la Dirección de Fomento, Iima, Perú, I9I I, año IX, núm. 7, pp. 65-84.

Cateriano, J. G. Informe sobre la plaga de langostas en el Transvaal y los medios empleados para combatirla. Anales de la Divección de Fomento, Lima, Perú, I9r2, año rgr2, núm. 6, pp. I-I9, I5 fig.

CAvarA, Fr. Le cavallette In Sardegna. Giomale di Agricoltura della Domenica, Piacenza, x 901 , anno XI, n. 29, pp. 228-229, fig.

Cecconi, G. Manuale di Entomologia forestale. Firenze, I9I4, fasc. I, pp. 4-6, fig. 2.

ChıттоN, E. Recherches sur l'action pathogène de divers coccobacilles sur le hanneton, le ver à soie, la cochylis et 1'eudémis. Annales du Service des Epiphyties, Paris, I9I3, t. I, pp. 37939 I.

Chatron, E. Septicémies spontanées à Coccobacilles chez le hanneton et le ver à soie. Comptes rendus hebdomadaires des séances de l'Académie des Sciences, Paris, I9I3, t. I56, $n^{\circ} 22$, pp. 1707 -I709.

Cimlaber'r, J. B. Instrucciones para combatir la langosta. Plan de campaña. Boletin del Departamento Nacional de Fomento, Asunción, Paraguay, I9I5, vol. II, núms. 8-9, pp. 6I-66.

"CICADA ». The locust or grasshopper plagues of Australia and modern methods of dealing with them. The Pastoralists' Review, Melbourne, I9I2, vol. IXII, no. 5, pp. 464-466, I fig.

CLIfTON, E;. Control of the black criket. Californian methods with grasshoppers. New Zcaland Department of Agriculiure, Industries and Commerce. The Journal of Agricultu,e, Wellington, I9I6, vol. XII, no. 3, pp. I87-IS9.

Comisión Central de extinción de la langosta [URUguay]. Memoria elevada al superior Gobierno por la - - - Invasión de I906-I90\%. MIontevideo, Imprenia "La Rural» de Eduardo Ramos, I907, 8 pp., I cuadro.

COMISIón CENTRAL DE EXTINCión DE LA LANGOSTa [URUGUAY]. Exhortación á los hacendados ! agricultores de la República. Irey del 27 de octubre de r9o8. Decreto reglamentario del 9 de noviembre de Igo8. Nombramiento de la Comisión central. Plan de defensa. Montevideo, Imprenta de la Buena Prensa, Igo8, 3 I pp.

Comisión de Parasitología Agrícola [Mexigue]. Destrucción de la langosta. Circulan 11úm. 6, México, s. d., pp. I-9.

CONOR, MI. Les invasions des santerelles en Afrique Mineure. (Figurations et textes anciens). Archives de l'Institut Pasteur de Tunis, Tunis, I9I6, t. IX, fasc. III, pp. I49-156, I fig., I pl.*

Cooke, C. J. Greece. Report for the year Igro on the trade and agriculture of the Piraeus and district. Diplomatic and Consular Reports. Annual Series, London, I 9r r, no. 4750, p. 9.

Cooley, R. A. First aumual report of the State entomologist of Iontana. Montana Agricultural College. Experiment Station. Bulletin no. 51, Bozeman, Mont., I904, pp. 232-242, fig. ro, pl. IV-VIII.

Cooley, R. A. Fourth annual report of the State entomologist of Montana. Montana Agricultural College. Experiment Station. Bulletin no. 64, Bozenan, Mont., rgo6, p. 43.

Cooley, R. A. Ninth annual report of the State entomologist of Montana. Montana Agricultwral College. Experiment Station. Bulletin no. 88, Bozeman, Mont., x 9x2, p. 87.

Cooley, R. A. Tentl annual report of the State entomologist of Montana. Montana Agricultural College. Experiment Station. Bulletin no. 92, Bozeman, Mont., x912, p. 52.

Corpeiro, V. A. Orthopteros de Setubal. Broteria, Serie zoologica, Braga, Igr 4, vol. XII, fasc. III, pp. $2 \mathrm{II}^{-2} \mathrm{I} 3$.

Cotes, E. C. Note on insects in India. Journal of Bombay Natural History Society, Bonbay, I 890, pp. 86-92.

Cores, E. C. Second note on locusts in India. Journal of Bombay Natural History Socicty', Bombay, I89o, pp. $184-\mathrm{I} 88$.

Cotes, E. C. The locust of North Westeru India. Joumal of Bombay Natural History Socicty, Bombay, I89I, VI, pp. $242-262$. 
Coupin, H. I,es santerelles. Chronique agricole du. Canton de Vaud, Lausanne, Igor, I4 ${ }^{\mathrm{e}}$ an., pp. $463-465$.

Couston, F. I,es santerelles. L,eur préférence alimentaire. Journal d'Agriculture pratique, Paris, I908, vol. 2, pp. 694-695.

CuBoni, G. Esperienze per la diffusione dell'Entomophthora Grylli Fries contro le cavallette. Nuvo Gionnale botanico italiano, Firenze, I889, vol. XXI, 1. 2, pp. 340-343.

Cullen, H. y MAgGio, C. F. Descripción de un nuevo cocobacilo patógeno para la langosta. Boletin del Ministerio de Agricultura, Buenos Aires, I9I2, tomo XIV, núms. II y I2, pp. I $368-$ 工 373,3 fig.

D'AlMem., J. Luta contra os gafanhotos na provincia de Moçambique. Revista Agronomica Lisboa, I9I5, ano XI ( $2^{\mathrm{a}}$. série), vol. 2, n.05 I3 a I6, Pp. II-30.

D.AMMERIIAN, K. W. Literatuur op het gebied van Dierkunde en Landbouwdierkunde, verschenen in Teysmannia van I89o (D1. I) tot einde I9I4 (D1. IXV). Teysmannia, Batavia, I9I5, 26 ste jaargang, $8^{\mathrm{e}}$ en $9^{\mathrm{e}}$ aflevering, blz. $5 \mathrm{I} 7,523-524,544-545,55 \mathrm{I}$.

DEAkin, R. II. Some East African insects of economic importance. The Annals of Applied Biology, Canibridge, I9I6, vol. II, no. 4, pp. 24 I-242.

DE Almeida e Brito, F. Invasão de gafanhotos. A Vinha Pontugueza, I,isboa, igr6, anno XXXI, n. ${ }^{\circ}$, p. I64.

DEAx, G. A. Grasshopper control work in Western Kansas. Joumal of Economic Entomology, Concord, N. H., I9I4, vol. 7, no. I, pp. 67-73, pl. I-2.

DEAx, G. A. Further data on poisoned bran mash flavored with fruit juice as a means of controlling some insects. Joumal of Economic Entomology, Concord, N. H., I9I5, vol. 8, no. 2, pp. $219-227$, passim.

Defensa Agrícola [ARgentine]. I,eyes 3708 y 4863. Reglamento é instrucciones sobre destrucción de langosta. Buenos Aires, Igo8, i 28 pp., 6 fig.

DE LA MARe Norris, F. I,ocust work in Selangor. Progress report for october. Agricultural Bulletin of the Federated Malay States, Singapore, I9I3, vol. II, no. 5, pp. I24-I25.

DE LA Mare Norris, F. Locust work in december. Agricultural Bulletin of the Federated Malay States, Singapore, I9I 4 , vol. II, no. 7, p. I 86.

DE LA MARE NorRis, F. Report on locust work. January, february and march, I9I5. The Agricultural Bulletin of the Federated Malay States, Singapore, I9I5, vol. III, no. 8, p1. $29 I-293$.

Del Guercio, G. Di una infezione crittogamica manifestatasi nel Caloptenus itilicus Burm., nella bassa pianura fiorentina. Bullttino della Societa botanica italiana, Firenze, I 894 , pp. 89-9I.

Departient of Agriculture, Bombay. The locust of the North-TVest. Leaflet no. I, Poona, Yeravda Prison Press, I9I5, 4 pp., 2 fig.

Department of Agriculture, British Columbia. Cutworms and grasshoppers. Method of prerention and destruction. (Extract from the report of M. R. PALMER, Inspector of Fruit Pests to the Honourable the Minister of Agriculture, I898-99). Victoria, I900, 3 pp., 6 fig.

DepartMenx of Agriculture, British EAst Africa. Leaflet no. 5, Mombasa, i905, $5 \mathrm{pp}$.

DE QueIroz Telles, A. Liquidos para a destruição de gafanhotos. Secretaria da Agricultura, Commercio e Obras Públicas do Estado de S. Paulo. Boletim de Agricultura, São Paulo, I9Io, I I série, n. Io, pp. 954-957.

DE SAINvIlle. [Invasion de sauterelles de I9I5 dans l'Afrique du Nord]. Bulletin de la Société Nationale d'Acclimatation de France, Paris, I9 16, 63 ${ }^{\text {e an., n }}{ }^{\circ}$ 7, pp. 286-287.

De Souza, P. Ia extinción de la langosta. Revista de la Asociación Rural del Uyuguay, Montevideo, I9I6, año XI,V, núm. „, p. 68.

Dessoliers, H. Abattoirs à sauterelles. Bulletin Agricole de l'Algérie-Tunisie-Maroc, Alger, I9I5, 2e série, $2 \mathrm{I}^{\mathrm{e}}$ an., $\mathrm{n}^{\mathrm{0}} 5$, pp. IO9-II 3 . 
De Stefani, 'T. Ie cavallettc in Italia. La Siciliu Agricola, Palerme, I888, an. YI, 11. I7, 1). 332 .

De Stefani, T. Una nota sulla Chalcis Dalmanni. Il Naturatista Siciliano, Palermo, I88g, all. IX, 21. I, Pp. II-I 2 .

DE Stefani, T. De duobus novis hymenopteris Siciliæ. Il Naturalista Siciliano, 1'alermo, Ibn , an. $\mathbf{X}, \mathbf{x .} 6, \mathrm{pp}$. II 7 -II 9 .

De Stefani, T. L'invasione delle cavallette. Gazzetta Comnerciale, Palermo, I9or, 1.. 6I3.

De Stefaxi, T. A propostito delle cavallette. Gazzetta Commerciale, Palermo, I $309,11.63$ I.

De Stefani, T. Intorno all'invasione di cavallette. Giornale di Sicilia, Palermo, Igog, an. XIIX, 11. I 52 .

De Stefant, T. Alcune notizie sulle carallette. Bollettino del R. Orto botanico e Giardino coloniale di Palemo, Palermo, I9IO, an. IX, fasc. I-2-3, P1. I 23-I25.

De Stefani, T. Un grave pericolo per l'agricoltura siciliana. Di nuovo le carallette. Giormale di Sicilia, Palermo, igro, an. I, n. I 2 .

DE Stefanr, T. Insetti criminali. A proposito delle recenti invasioni di cavallette in Sicilia. La Scienza per tutti, Milano, I9I I, au. XVIII, 11. 66, pp. 3I9-320.

De Stefani, T. Le cavallette ed alcuni loro parassiti. Osservazioni fatte negli anni I9IO-IgI I durante l'invasione della provincia di Palermo. Bollettino del Ministero di Agricoltura, Industria e Commercio, Roma, I9I2, an. II, serie C, fasc. 2-3, pp. 30-52, fig. I-IT, tav. I-IV.

De Stef.ji, T. Insetti occasjonalnente dannosi alle viti. Palermo, Tip. G. Di Giorgi, IgI 4 , pp. $7-8$

De Stefani Perez, T. Cavallette, loro invasioni e lot ta contro di esse in Sicilia. Osservazioni fatte durante 1'invasione della provincia di Palemo negli anni I9Io-I9I I. Giomale di Scienze Naturali ed Econoniche, Palermo, I9I3, vol. XXX, pp. II7-I99, fig. I-2I.*

DE TIGNy, F. M. G. T. Histoire naturelle des insectes. Paris, Verdière, I $823, \mathrm{t} . \mathrm{T}, \mathrm{pp} .47-68$, fìg.

D'Hérelle, F. Sur une épizootie de nature bactérienne sévissant sur les sauterelles au IIexique. Comptes rendus hebdomadaires des séances de l'Académie des Sciences, Paris, I9I I, t. I52, $\left.11^{\circ} 2 \mathrm{I}, 1 \mathrm{I}\right)$. I 4 I 3 -I 4 I 5 .

D'Hérelle, F. Sur la propagation, dans la République Argentine, de l'épizootie des sauterelles du Mexique. Comptes rendus hebdomadaires des séances de l'Académie des Sciences, Paris. I9I 2 , t. I $54,11^{\circ} 9$, pD. $623-625$.

D'Hérelle, F. La langosta. Revista Agricola Salvadoreña, S. Salvador, I9I4, año II, 11. Io, p1. 305-310.

D'Hérelle, F. I, caccobacille des sauterelles. Amales de l'Institut Pasteur, Paris; I9It, 1. XXVIII, $\mathbf{1 1}^{0} 3$, pp. $280-328$, fig. I-4; $n^{6} 4$, pp. $387-407$, I fig.

D'Hérelle, F. La campagne contre les santerelles en Tunisie ell I9I 5. Bullitin di la Socicti de Pathologic exotique, Paris, I9 5, t. VIII, $11^{\circ}$ 9, pp. 629-633.

D'Hérelle, F. Sur le procédé biologique de destruction des sauterelles. Comptcs rudus hebdomadaires des séances de l'Académie des Sciences, Paris, I9I 5, 1. I6I, $1^{0}$ I7, p1). 503-505.

D'Hérelle, F. Campagne contre les Schistocerca peregrina en Tuuisie par la méthode biologique (avril- juillet I9I5). Archives de l'Institut Pasteus de Tunis, Tunis, I9I6, t. IX, fasc. III. Pl. I 3.5 -I 48 .

DIRECTION GÉNÉRALE DE L'AgRICULTURE [TUNISIE]. I,es sauterelles. Revue Tumisienni, Tunis, I9I6, XXIII an., no II6, pp. I55-I90, fig. I-I6.

Doello Jurado, M. I a región permanente de la langosta. Revista de la Asociación Rural del. Uruguay, Montevideo, I9I I, año XI, n. ${ }^{\circ}$, pp. $226-233$.

DoLfi, A. Guerra alle cavallette. Un iluovo apparecchio per la cattura del vorace ortotiero. Giornale di Agricoltura della Domcmica, Piacenza, I9 Io, an. XX, n. 40, 1) 374. 4 fig.

DoRIA, I, Origine propagazione, e danni delle locuste. Operazioni praticate per la loro est irpazione noll'Agro Ronano, ed in varj altri territorj dal i 807 all'anno I I $_{5}$. Xatura, c pro- 
prietà di tali insetti. L,cggi decisioni, e divisione delle spese. Roma, Crispino Puccinelli, I 8 I6, passim.

Doten, S. B. Grasshoppers in alfalfa fields. University of Nevada, Rcno, Nevada. Agricultural Experiment Station. Bulletin no. 57, Reno, Nevada, I904, 4 pp., 2 fig.

DrIEBERG, C. Locust fungus. The Tropical Agriculturist, Colombo, rgor, vol. XVIII, p. 656.

D'URBAN. Die Wanderlieuschrecke Pachytylus migratorius in Istrien. Centralblatt fiur das Forstwesen, I880, VI, S. 446 .

D'Utra, G. O problema da extincção dos gafanhotos. Secretaría da Agricultura, Commercio e Obras Públicas do Estado de S. Paulo. Boletim de Agricultura, São Paulo, I9ro, I I serie, n. i I, 2, 3, p1. 3-I5, 8I-90, I6I-I70.

F.Astham, J. W. and Ruhmaxx, $M_{\mathrm{Ax}} \mathrm{H}$. Diseases and pests of cultivated plants. Department of Agriculture (Horticultural Branch). Bullein no. 68, Victoria, B. C., I9I6, p. 62.

EssIG, E. O. Injurious and beneficial insects of Califormia. The Monthly Bulletin of State Commission of Horticulture, Saeramento, California, I9I3, vol. II, nos. I and 2, pp. I3-20, fig. $12-18$.

FAIvre, W. Notice sur les moyens d'organiser la lutte contre les criquets. Bulletin de la Direction. de l'Agriculture, du Conmerce et de la Colonisation, Tunis, Igro, ${ }_{2}^{\mathrm{e}}$ trim., pp. 3-I2 du tirage a part.

FELT, E. P. $30^{\text {th }}$ Report of the State entomologist on injurions and other insects of the State of New York, I9I4. University of the State of New York. Bulletin no. 606 (Muscum Bulletin I 80), Albany, rgr5, pp. 46-57, pl. I.

FELr, E. P. Grasshopper control in New York State. Journal of Economic Entomology, Concord, N. H., I9I 5 , vol. 8 , no. 2 , pp. $22 \%-230$.

FLetcher, J. Report of the entomologist and botanist, rgor. Canada. Department of Agriculture. Central Experiment Farm, Ottawa, I902, pp. I97-262, passim.

FLeTcher, J. Inseets injurious to grain and fodder crops, root crops and vegetables. Canada. Department of Agriculture. Experimental Fann. Bulletin no. 52, r905, pp. 2 2-22.

Fletcher, T. BAINBrigge. Note on insects attacking the paddy plant in Southern India. Ticpartment of Agriculture, Madras. Bulletin no. 67, I9I3, pp. I-3, fig. I-2, pl. I.

Fletcher, T. BaINBrigge. List of insect pests of cultivated plants in sonthern India. Coimbatore, Agricultural College and Research Institute, I9I3, p. I 8.

FLETcher, T. BAINBRIGge. I,ist of insect pests of cultivated plants in Southern India. Department of Agriculture, Madras. Note uo. I of I9I3, Coimbatore, I9I3, p. I5.

Fletcher, T. BAIndrigge. Grasshoppers. Madras Agricultural Calendar, r9r3-I4, p. I7, I fig.

FLETcher, T. BaINBrigce. Some South Indian insects and other animals of importance considered especially from an economic point of view. Madras, I9I 4, pp. 524-533, fig. 4I6-426, pl. XIVIII-I.

FRENCH, C. A handbook of the destructive insects of Victoria, with notes on the methods to be adopted to check and extirpate them. Melbourne, Robt. S. Brain, I90o, part. III, pp. 27-38, 203-208, fig. 15-26, pl. XXXVII-XXXVIIA.

FrogGatt, W. W. Entomological work and notes for 1900. Miscellaneous Publication no. $48 \%$, Sydney, William Applegate Gullick, Igor, pp. 2-5.

Frogg.4T, WW. W. The eastern plague locust (Oedaleus senegalensis, Krauss.). Some suggestions how to check them. Miscellaneous Publication no. r,095, Sydney, Willian Applegate Gullick, I907, 3 pp., I pl.

FroGgatr, WV. WV. Locusts in Anstralia and other countries. Department of Agriculture, Neat South Wales. Farmer's Bulletin no. 29, Sydney, Igro, 40 pp., I3 fig.*

FRoGGatT, W. W. Formula for spraying locusts and their food, to kill them before they become winged. Department of Agriculture, Sydney, William Applegate Gullick, I9II, I p.

Froggatt, W. W. Destruction of locusts. The Agricultural Gazette of Nez! South Wales, Sydney, I9I2, vol. 23, p. I 46 . 
Froggate, W. W. Presidential address. Proceedings of the Limnean Socicty of New South Wales rgI2, vol. XXXVII, part 5, pp. 23-24.

Froggatt, W. W. Pests and diseases of the coconut palm. Department of Agriculture, Nicw South Wales. Science Bulletin no. 2 (third edition, revised and enlarged), Sydney, rgr 4 , p. $42 *$.

Fuller, C. First report of the Committee of control of the South African Central Locust Bureau. Cape Town, Townshend, Taylor and Snashall, I907, II2 pp., I pl.

Fuller, C. Second annual report of the Committee of Control of the South African Central Locust Bureau. Cape Town, Cape Times Limited, Igon, $86 \mathrm{pp}$.

Galdardo, A. I,a lucha científica contra las plagas. La Agricultura Nacional, Buenos tires, I908, año I, núm. 6, pp. 494-497.

Galfardo, A. La destrucción de la langosta por sus enemigos naturales. Anales del Museo Nacional de Historia Natural de Buenos Aires, Bumos Aires, I9I2, tomo XXIII, pp. $15.5-$ $165 *$.

GARDÈ;, J. Y. Os gafanhotos em Matto-Grosso. Ares destruidoras de saltôes. Chacaras e Quintaes, S. Paulo, Brazil, I9II, vol. III, n. 5, p. 25.

Garmax, H. Destructive locusts in Kentucky. Kentucky Agricultural Experiment Station of the State College of Kentucky. Bulletin no. 49, Lexington, Kentucky, I 894, pp ;-22, fig. I-6.

GaUmer, G. F. Parásitos de la langosta. El Agricultor Mexicanio, C. Juárez, IgIr, t. XXXI, n. I, pp. 2-4.

Geismar, I. M. Grasshoppers and their control. Michigan A gricultural College Experiment Station. Special Bulletin 53, East Lansing, Michigan, I9ro, 7 pp.

Gmigr, A. Materiali per lo studio della fama libica. Memorie della R. Accademia delle Scicnze dell'Istituto di Bologna, Classe di Scienze fisich; Bologna, I9I3, serif II, tomo X, IgI2г913, pp. 253-296, passin*

Giacalone, S. Contro le cavallette nella prossima stagione. Vomerc, Giomale agricolo-commerciale della provincia di Trapani, Marsala, I9Io, an. XV, n. 707.

GIARD, A. Le champignon parasite des criquets. LaNatuve, Paris, I89I, t. XIX, nº. 956, p. 270 .

GIARD, A. Observations et expériences sur les champignons parasites de l'Acridium percgnnum. Comptes rendus de la Socićté de Biologie, Paris, I891, vol. XLIII, p. 493.

GIARD, A. Le criquet pèlerin (Schistocerca peregrina Ol.) et son cryptogame parasitc, Lachnidium acridionun. Comptes rendus de la Société de Biologie, Paris, I89z, vol. SI,IV, p. 2.

Grard, A. Nouvelles études sur le Lachnidium acridionm Giard, parasite du criquet pèlerin. Revue générale de Botanique, Paris, I892, t. IV, $\mathrm{n}^{\circ} 47, \mathrm{pp} .449-46 \mathrm{I}$, I pl.

GraRD, A. Réponse à $\mathrm{M}$. Ch. Brongniart relativement au champignon du criquet pèlerin. Bulletin de la Société cntomologique de France, Paris, I892, t. LXI, p. LXXXIT.

Grbsox, A. Reports ou insects of the year. Division mo. I. Forty-Fifth Ammal Report of the Entomological Society of Ontario, I914, Toronto, I9I5, p. I4.

Gibsox, A. Experiments with poisoned bran baits for locust control in Eastern Canada. Forty-Fifth Annual Report of the Entomological Society of Ontario, I914, Toronto, I915, pp. 97-I02, fig. I9.

Gibsox, A. Locust control work in Fastern Canada in 1915. The Agricultural Gazette of Canada, Ottawa, I915, vol. 2, no. ro, pp. 937-940, 2 fig.

Gibson, A. The control of locusts in Eastern Canada. Dominion of Canada. Department of Agriculture. Entomological Branch. Circular no. 5, Ottawa, Ont., I9I 5, 8 pp., 6 fig.

Gilchrist, J. D. F. Agricultural zoology for South African students. The Agricultural Joumal. of the Cape of Good Hope, Cape Town, I9I0, vol. XXxvI, no. I, pp. 47-52, fig. 68-j2.

Gillette, C. P. Grasshopper conditions in Colorado. Joumal of Economic Entomolog1; Concord, N. H., I9I2, vol. 5, 110. 2, pp. I2I-I23.

GrRaUlt, A. A. Notes on a plague of locusts in North Queensland, and its relation to sugar cane. Societas Entomologica, Stuttgart, I9I3, vol. XXYYIII, n. II-I2, pp. 45-46, +9-50. 
GIRAUlt, A. A. A new scelionid parasite of locust eggs irom the wortherm territory of Australia. Entomologist, I,ondon, I9I4, vol. XIVII, p. 197.

Girault, A. A. A few notes on Queensland insects. Entonological News, Philadelphia, I9I5, vol. XXVI, no. 8, p. 362 .

Godor, C. A praga dos gafanhotos de I915-1916. Secretaria da Agricultura, Commercio e Obras

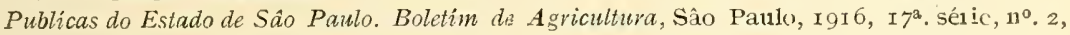
pp. I30-I35.

Gonzales Montaner, I. Cartilla de la Defensa Agricola ordenadamente calcada en las leyes, reglamentos, disposiciones vigentes y observaciones cientificas sobre la destrucción de la langosta. Buenos Aires, I9o8, pp. I-I 6 .

Goxz.1LEz, B. Informes sobre destrucción de la langosta. Rcvista A grícola, Bogota, I9 I 5, vol. I, n. 4 , pp. 2 I0-2II.

Gowder, C. C. Uganda insect pests. Cotton Department. Entomological Leaflet 11o. 2, Uganda, I 909, p. 5 .

Grt.vol, G. Dispense di Entomologia agraria secondo le lezioni del Prof. F. SilvestrI. Portici, Stab. tip. Vesuviano di E. Della Torre, I9II, Parte speciale, pp. I3-38, fig. 5-28.

GREEN, E. E. The spotted locust (Aularches miliaris, I.). Circulars and Agricultural Jourmal of the Royal Botanic Gardens, C-ylon, Peradeniya, I906, vol. III, no. I6, pp. 227-236, I pl.

Guésaux, G. Entomologie et Parasitologie agricoles. [Eucyclopédie agricole]. $2^{\mathrm{e}}$ éd. Paris, Librairie J.-B. Baillière et fils, I9I0, pp. III-I22, fig. 82-88.

GuÉrin, R. La destruction des sauterelles par le procédé d'Hérelle. Journal d'A griculture tropicale, Paris, I912, 12 e an., no 129 , pp. 70-72.

Gú́ris, R. Procédé biologique de destruction des sauterelles. La Naturc, Paris, x9I2, $40^{\mathrm{e}}$ an., 2ème sem., $\mathrm{n}^{0} 2057$, pp. 34I-343, fig. I-3.

Guillochon, I. Note sur la taille à appliquer aux plantes arbustives endommagées par les criquets. Bulletin de la Direction générale de l'A griculture, du Commerce et de la Colonisation, Tanis, I9I5, I9 $\mathrm{e}$ an., no 84 , pp. 2 I 8-220.

Guillon, J et Perrier de la Batmie, I. I es criquets dans les Charentes. Revue de Viticulture, Paris, I 902 , vc1. I\%, pp. 653-659; vol. I8, pp. 6I-64, I2 fig.

Gutmarães, R. A praga dos gafanhotos de igi5-Igi6. Secretaría da Agricultura, Commercio e Obras Públicas do Estado de São Paulo. Boletím de Agricultura, São Paulo, I916, 17. ${ }^{2}$ série; $\mathrm{n}^{\circ}$. 2, pp. I35-I43.

Gumiarães, R. F. Relatorio do Inspector Agricola - - -, correspondente ao anno de I9I5. Secretaria da Agricultura, Commercio e Obras Públicas do Estado de São Paulo. Boletim de Agricultura, São Paulo, I9I6, I7.$^{a}$ séric, n. ${ }^{\circ} 2$, p. I50.

GunNing, J. W. Iocusts birds: The Transvaal Agricultural Journal, Pretoria, 1908, vo1. 6, pp. 52 -530 .

Gurney, W. M. B. Notes on grasshopper (or locust) swarms in New South Wales during I9o7-8. The Agricultural Gazette of New South Wales. Miscellaneous Publication no. I, I57, Sydney, เ908, 9 pp., 6 fig., I pl.

Guzmáx, J. Fl maíz, tabaco, caña de azucar, cocotero, ayote. Estudios de Patología regetal en El Salvador. Revista Agrícola Salvadoreña, San Salvador, I91 5, año III, nº 3, pp. 74-76.

Gvozdenović, F. L'invasione delle cavallette sul Carso e modo di combatterla durante l'invenno. Gorizia, Stab. tip. Giov. Paternolli, rgo8, 8 pp., 7 fig.

Gvozdenović, Fr. Die Heuschrecken-Bekämpfungsaktion am Karste im Sommer rgog. Zeitschrift für das landwirtschaftliche Versuchswesen in Oesterreich, Wien, I9I0, Jahrg. I3, S. 699-74I, Fig. I-8.

Gvozdexović, Fr. Beobachtungen über den Stand der Heuschreckeninvasion am Görzer Karste im Jahre I9I0. Zeitschrift fïr das landwirtschaftliche Versuchswesen in Oesterrich, Wien, I9I0, Jahrg. I3, S. 957-959.

Gvozpexović, Fr. Der Einfall der Heuschrecken im Karstgebiete und die Weise ihrer Bekämprung während des Winters. Görzer agric.-chemische Versuchsanstalt, Görz, I9ro, 8 S., 8 Abb. 
GvozDexović, Fr. Der Kampf gegen die Henschrecken im Karstgebiete während des Sommers 1909. Görzer agric.-chemische Versucilsanstalt, Görz, 19I0, I 8 S., 8 Abh.

Gvozdenović, Fr. La lotta contro le cavallette sul Carso nell'estate rgog. Relazione di--. L'A mministrazione Autonomx, I909, Gorizia, I9Io, n. II-I2, pp. I-I 8 du tirage à part, fig. $x-8$.

Gvozdenović, Fr. O pokončeranju kobilic na Krasu r letn I909. Spisal- -. Samonpraté, Gorica, xqI0, štev. II-I2, pp. I-I 7 du tirage à part, fig. I-8.

Hasemax, L. Insect pests of field crops. University of Hinnesota. Agricultural Experiment Station. Bulletin no. I34, Columbia, Missouri, 1915, pp. 34-35, fig. 3.4.

Hebard, M. Records of Orthoptera from Newfoundland. Entomological New's, Philadelphia, I 9 I 5 , vol. XXVI, no. 7, p. 306.

Herfa, Percy A.- Henry, G. M. Locusts. The Tropical Agriculturist: Journal of the Ceylon. Agricultural Society, Colombo, 1916, vol. XIVI, no. 6, pp. 365-366.

Hewrtt, C. G. Importance de l'entomologie dans le développement du Canada. Ottana, C. I. Parmelee, I9Io, p1. $48-49$.

Heivitr, C. G. Inportance of entomology in the development of Canada. Ottawa, C. H. Parmelee, I9го, pp. $45-46$.

Hewitt, C. G. The control of grasshoppers and locusts. Departnicnt of Agriculture, Canada Census and Statistics Monthly, I912, vol. 5, p1. 157-158.

Hotlrung, M. Jahresbericht über die Neuerungen und Leistungen auf den Gebiete des Pflanzenschutzes. Berlin, P. Parey, I900, I902, Bd. II-III, passim.*

Holtruvg, M. Jahresbericht über die Neuerungen und Leistungen auf dem Gebicte der Pflanzenkrankheiten. Berlin, P. Parey, I903, I904, I905, Bd. IV-VII, passim**

HoLlRung, M. Jahresbericht über das Gebiete der Pflanzenkrankheiten. Berlin, P. Parey, I907-1913, Bd. VIII-XIV, lassim*.

Holman-Hunt, C. B. Report on some Indian locusts. The Agricultural Bulletin of the Federated Malay States, Singapore, 1912, vol. I, no. I, pp. I2-13.

Holman-Hunt, C. B. Notes on insect pests. The Agricuitural Bulletin of the Federated Malay States, Singapore, I9I3, vol. I, no. 9, p. 327.

Holman-Hunt, C. B. Notes on insect pests. The Agricultural Bulletin of the Federated Ialay States, Singapore, 1913, vol. I, no. 10, pp. 368-369.

Homolne, J. Ettude sur l'invasion des criquets pèlerins dans la région de Sidi-Ferruch. Alger, Imprim. Algérienne, I908.

Houlbert, C. Les insectes. Anatomie et physiologie générales. Introduction à l'étude de l'Entomologie biologique. Paris, O. Doin et fils, 1910, passim.

Houser, J. S. Grasshoppers. Ohio Agricultural Experiment Station. Circular I37, Tooster, Ohio, I913, pp. 127-134, fig. I-10.

Howard, C. WV. Insectos e mais nocivos parasitas. Relatorio do Chefe da Secção de Entomologia Secretaria Geral do Governo da Provincia de Moçambique. Bolctim da Repartiçäo de Agricultuva, Louren ço Marques, 1910, $11^{\circ}$. 2, pp. Io6-ro7.

Howard, C. W. A destruição dos gafanhotos ua Africa do Sul. Secretaria Geral do Governo da. Provincia de Moçambique. Boletim da Repartição de Agricultura, Lourenço Larques, Igro, $\mathrm{n}^{\mathrm{o}}$. 4, pp. 255-266.

Hunter, J.S. Studies in grasshoppers control. University of California Publications. College: of Agriculture. Agricultural Experiment Station. Bulletin no. I70, Sacramento, 1905, 23 pp., I8 fig.

Hunter, S. J. and ClaAssen, P. W. Grasshopper control in the Southern Division of Kansas. Journal of Economic Entomology, Concord, N. H., I9I4, vol. 7, גo. 1, pp. 73-83, p1. 3-5*.

Institut international D'Agriculture. Anuuaire international de législation agricole. Rome, I9I2-I916, Ière-Vème année (I9II-I9I5), passim.

Institut Intersational d'Agriculture. Actes de la Conférence interiationale de Pliytopathologie, 24 férrier-4 mars 1914 . Rome, I91.1, passim. 
INSTITUT LNTERNATIONAL D'AGRICULTURE. BUREAU DES RENSEIGNEMENTS AGRICOLES ET DES MALADIES DEs PLANTES. I,e service de protection des plantes dans les divers pays. IIrème édit. Rome, r9r4, passim.

JAbloxowski, J. A nagyhortobágyi sáskairtás eredınényei. Budapest, A Pesti L,loyd-Társulat, Könyvsajtója, r9ro, I 5 lap., 5 ábra.

JAblonowski, J. A nagykörüi sáskairtás. Mezögazdasagi Szemle, Budapest, I9I4, XXXII.

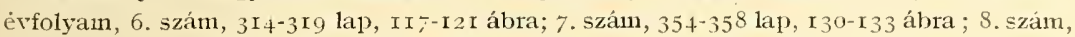
394-399 lap, r39-r 4 ábra ; 9-Ir. szám, 447-459 lap, I6I-I62 ábra.

TARRotr, R. Grasshoppers. The Queensland Agricultural Joumal, Brisbane, IgI2, vol. XXYIII, pp. I69-I 70 .

J.ARVIS, E. Notes on insects damaging sugar-cane in Queensland. Queensland. Bureau of Sugar Experiment. Stations. Division of Entomology. Bulletin no. 3, Brishane, r9r6, 11. I7-22, p1. III, fig. I.1-I5.

Kellogg, V. I. American insects. Third edition, revised (American Nature Series, Groul' I. Natural History). New York, Henry Holt and Company, I9 14, pp. I23-I47, fig. I55-I 56, I 65 -I 93 .

KELLI, E. O. C. A new sarcophagid parasite of grasshoppers. Joumal of Agricultural Research, Washington, D. C., I9I4, vol. II, no. 6, pp. 435-445, p1. XI.

KEuchenrus, P. E. Ziekten en piagen van de klapperkultuur in Nederlands-Indië. Teysmannia, Batavia, I9 I 5, 26ste jaargang, Io ${ }^{\mathrm{e}}$ afleveriug, blz. 605 .

Kevchenrus, P. F. Ziekten en plagen van de klapperkultuur in Besoeki en de middelen ter bestrijding. Mededeelingen van het Besoekisch Proefstation, s. d., No. 20, blz. 5.

KIRBY, W. F. A synonymic catalogue of Orthoptera. London, I906 - I9Io, vol. II : Orthoptera saltatoria, part I (Achetida et Phasgonurida), Vol. III: Orthoptera saltatoria, part II (Locustide vel Acridida), passim.

Kólle, S. W. African native literature of the Kanur or Bornu language. Church Missionary Society, 1854, p. $x 98$.

KoxIxGsBergen, J. C. Tweede overzicht der schadelijke en nuttige insecten vau Java. M 1 dedeelingen uitgaande van het Departement van Landbouw, No. 6, Batavia, xgos, blz. $60-62^{*}$.

KRAUS, R. Versuche zur Bekämpfung der Heuschrecken mittels des Coccobacillus acridiorum d'Hérelle in Argentinien. Centralblatt für Bakteriologie, Parasitenkunde und Ințektionskrankheiten, Jena, I9r6, 45. Bd., No. I8/25, S. 594-599.

Kraus, R., I Ahille, F., Morales D., MagGio, C. A. Informe de la Comisión designada por el Ministerio de Agricultura para estudiar la eficacia del cocobacilo acridiorum d'Hérelle como medio para la destrucción de la langosta. República Argentina. Boletin del Ministerio de Agricultura de la Nación, Buenos Aires, I915, tomo XIX, núms. ro-I:-12, pp. $827-849 *$ *

KRAUSSE, A. H. Heuschrecken auf Sardinien. Zeitschnft für wissenschaftliche Insehtenbiologit, Berlin-Schöneberg; I912, 8. Jahrg., S. 323-326.

KÜNCKEL D'HerculaIs, J. Invasions des acridiens, vulgo sauterelles, en Algérie. Alger-Mustapha, Girault, I893-r905, t. I-II, avec fig., pl. et cartes, passin*.

Küxckel d'Herculats, I. [I,es invasions de sauterelles dans l'Afrique du Nord, et les moyens de défense et de destruction]. Bulletin de la Sociéié Nationale d'Acclimatation de France, Paris, rgr6, $63^{\mathrm{e}}$ an., $11^{\circ} 7, \mathrm{pp} .285^{-286}$.

KüNSTLER. Über Heuschreckenfrass. Verhandl. zool.-bot. Ges., Wien, I86 +, XIT, S. 769-776.

IA BAUME, W. Die Bekämpfung der Wanderheuschrecken in Sudafrika. Prometheus, Berlin, I9I2, XXII1. Jahrg., Nr. Ir76, S. 497-502.

I, A BAume, W. Die afrikanischen Wanderheuschrecken. Beiheftc zum Tropenpflanzer, Berlin, Igro, Bd. XI, Nr. 2 , S. 65-I29, Abb. I-Io, Taf. I-IY*.

IA BAUME, W. L,e cavallette africane (Die afrikanischen Wanderleuschrecken). Prina traduzione italiana autorizzata di A. Moreschini. Novara-Roma, Istituto geografico De Agri- 
stini, I9I3, 78 pp., Io fig., IV tav. (X. 8 della "Biblioteca agraria coloniale "dell'Istituto agricolo coloniale italiano, diretta da G. BARTolommeI-GIOLI).

I.AHILle, F. I,a langosta y sus moscas parasitarias. República Argentina. Anales del Ministerio de Agricultura. Sección de Zootecnia, Bacteriología, Veterinaria y Zoología. Buenos Aires, I907, tomo III, núm. 4, pp. I-I36, fig. 1-29, lám. I-VII.

IEA, A. M. Insect and fungous pests of the orchard and farm. Third edition. Hobart, Tasmania, John Vail, x 908 , pp. $76-7 \%, 2$ fig.

LEFroy, H. M. Indian insect pests. Calcutta, Government Printing, Igo6, chapter XVIII, pp. $214-220$.

I,EFroy, H. M. The Bombay locnst. A report on the investigations of I903-04. Memoirs of the Department of Agriculture in India. Entomological Series. Calcutta, rgo6, vol. I, no, I.

I EFroy, H. M. Iocusts in India. Agricultural Joumal of India, Calcutta, r9o7, rol. II, part 3 , pp. $237-24.5,7 \mathrm{pl}$.

LEFROY, H. M. The more important insects injurious to Indian agriculture. Memoirs of the Department of A griculture in India. Entomological Series. Calcutta, I907, vol. I, no. 2.

LeFroy, H. M. Locusts in India. Deparment of Agriculture, Bombay. Bulletin no. 32, Bombay, I9os, pp. I-I2, p1. XIV-XX.

Lefroy, H. M. and Howlett, F. M. Indian insect life. Calcutta-Simla, Thacker, Spink \& Co., I909, pp. \} _ { 4 } - 8 9 \text { , fig. I9-29, pl. II-VII. }

LEnt́e, C. La langosta. Sus costumbres; su extinción. 2a edición ilustrada. Ia Plata, Sesé y Larrañaga, I906, r oo pp., 7 fig., x mapa.

L,EONARDI, G. Gli insetti nocivi ai nostri orti, campi, frutteti e boschi, all'uomo e agli animali domestici. Loro vita, danni e modi per preveniri. Neurotteri, emitteri, fisapodi, ortotteri e pseudoinsetti. Dans $\mathbf{I}_{\mathbf{a}}$ scienza e la pratica dell'agricoltura esposte e coordinate. Napoli, E. Marghieri, I9or, Tol. XI, parte IV (vol. IV), pp. 680-755, Sor-8II, fig. 228$274^{*}$.

LEst.AGE. L,es acridiens dans la commune mixte d'Aïn-Bessem, i 888-I 889. Blidah, Imprim, admin. A. Manguin, I889.

I,ETOURnead, F. Guerte aux sauterelles. Le Joumal d'Agriculture et d'Horticulture illustré, Québec, I916, vol. 19, $\mathrm{n}^{\circ}$ 12, p. 252, I fig.

Lewton-Brain, I. Agriculture in Malaya in I9I3. Departement of Agriculture, Federater Malay States. Bulletin no. 20, Singapore, I9I4, pp. 18-22.

LochHEAD, W. Brief notes on some of the injurious insects of Quebec, I914. Fonty-Fith Annual Report of the Entomological Society of Ontario, I9I 4, Toron1o, r915, p. 60.

LocmHEAD, W. Principaux insectes nuisibles de I91. Septitme rapport annuel de la Sociití de Québec pour la protection des plantes contre les insectes et les maladies fongueuses, IOI4-I9I 5 . Québec, E. E. Cinq-Mars, I 915 , p. I25.

L,ORENA, B. Relatorio sobre o serviço de destruiçãn de gafanhotos, apresentado pelo Ispector Agricola - - Secretaria da Agricultura, Commercio e Obras Públicas do Estado de São Paulo. Boletim de Agicultriva, São Paulo, Igr6. I7. ${ }^{a}$ série, n ${ }^{\circ}$ 2, p1. I.43-I47.

LorenA, B. Relatorio dos serviços de que foi encarregado o Inspector Agricola - -, durante o anno de I915. Secretaría da Agricultura, Commercio e Obras Públicas do Estado de São

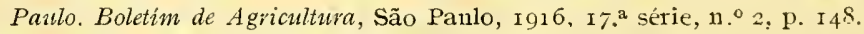

Lounsbury, Chas. P. The locust plague. The Agricultural Journal of the Cape of Good Hope, Cape Town, r9o\%, vol. XXXI, no. 2 , pp. I68-I I. $^{\circ}$

Lounsbury, Chas. P. Third annual report of the Committee of Control of the Sonth African Central Locust Bureau. Cape Town, Cape Times Linited, Goremment Printers, Igog, $68 \mathrm{pp}$.

Lounspury, Chas. P. Fourth annuel report of the Committee of Control of the South African Central Locust Bureau. Cape Town, Cape Times Limited, Gorernment Printers, IgIC, 59 pp., 2 pl., I 5 maps.

Iounsbury, Chas. P. Bericht der Regierungsentomologen in Kapstadt fiur das Jalı I 909. 
Centralblatt für Baktcriologic, Parasitenkunde $u$. Infcktionskrankhciten, Jena, I9I I, II. Abt., 30. Bd., No. I9/20, S. $45 \mathrm{I}-462$.

Lounsbury, Chas. P. Division of Entomology: annual report, I9I I. (Appendix XVII). Pretoria, I9I2, Pp. 13-I4.

Lounsbury, Chas. P. Locust bacterial disease. The Agricultural Journal of the Union of South Africa, Pretoria, I9I3, vol. V, 110. 4, Pp. 607-6II.

L,ounsburr, Chas. P. Division of Fintomology: annual report, I9 3 -I 4 (Appendix X). Pretoria, I $9 \mathrm{I}_{4}$ (?), pD. I3-I8.

L,ounsburr, Chas. P. Some phases of the locust problem. The South African Joumal of Science, Cape Town, I9I 5, vol. XII, no. 2, pp. 33-45.

Lugger, O. The Orthoptera of Minnesota. Thisd Anmual Report of the Entomologist of the State Expcriment Station of the University of Minmesota for I 897, St. Paul, I898, 285 11., I 87 fig., passim.

Lunardoni, A. Le cavallette. Loro vita, danui e modi per prevenirli. Rona, Tip. Tiberina di F. Setth, r888, passim.

LunARdoxi, A. Comment on combat les sauterelles en Italie. Institut international d'Agriculture. Bulletin mensuel des renseignements agricoles ct des maladies des plantes, Rome, I9I5, $\mathrm{VI}^{\mathrm{e}}$ an., $\mathrm{n}^{\circ} 4$, pp. 550-562, 2 pl.; et dans ses Editions italienne, anglaise, allemande, espagnole.

LUNARDoni, A. Relazione sulla lotta contro le cavallette. Bollettino del Ministero di Agricoltura, Industria e Commercio, Roma, I9I 5, an. XIV, vol. I, serie $B$, fasc. 5-6, pp. 258-270, tav. IVIII.

L,YNCh ARRIBÁlzaga, E. I a langosta voladora del Perú. Anales del Museo Náacional de Historia Natural de Buenos Aires, Buenos Aires, I903, serie III, tomo II, pp. I-6.

L, YNCH ARRIBÁlZAGA, E. Informe sobre una investigación realizada en Bolivia acerca de la región permanente de la langosta voladora. Buenos Aires, I9Io, Io8 pp., 2 mapas.

M. E sempre le cavallette ' Giornale d'Agricoltura della Domenica, Piacenza, I9Io, an. XX, I1. I 8 ; p. 167 .

M. Per una lotta efficace contro le cavallette. Gionnale di Agricoltr ra della. Domenica, Piacenza, I 9 Io, an. XX, n. 22 , p. 203.

MAGYAR KIRÁLYI FöLDMIVELÉSỨYYI MINISTER. Valamenuyi vármegyei és városi törvènyhatóságnak. Budapest, Franklin Társulat nyomdája, I907, 28,000, VI/3, I907: 4 lap.

Majorana, F. Sulla invasione delle cavallette nelle regioni delle Madonie. Gionnale della Commissione di Agricoltura e Pastorizia in Sicilia, Palermo, $1858,2^{a}$ serie, rol. II, fasc. Io, pp. 33-52.

Mally, C. W. Division of Entomology : annual report, I9I2-I9I3. (Appendix XIII). Pretoria, I9I3, p. 20.

Mancheron, P. La lutte contre les criquets dans la commune mixte du Djebel Nador. Reuze Agricole et Viticolc de 'A Arique du Nord, Alger, I9I4, vol. III, no II 4, pp. 460-46I.

MarchaL, P. Les sciences biologiques appliquées à l'agriculture et la lutte contre les ennemis des plantes, aux Etats-Unis. Annales des Épiphyties, Paris, I916, t. III, pp. 55, 346, 347*.

MARRE, E. Invasion de criquets dans l'arrondissement de Saint-Afrique. (Etude sur le criquet italien). Le Progrès Agricole ct Viticole, Montpellier, rgor, i $8^{\mathrm{e}}$ a11., vol. 35, pp. 464-472.

Marre, E. Les sauterelles dans l'Aveyron. Procédé de destruction. Le Progrès A gricole et Viticole, Montpellier, I9or, I8e an1., vol. 36, pp. I03-108.

Martell, G. L,e cavallette. $R$. Scuola superiore d'A gricoltura in Portici. Bollettino n. I, serie IY, Portici, I909, 20 pp., I 6 fig.

Martell, G. Breve risposta ad alcune critiche ai mezzi di lotta contro le cavalle1te. A gricoltore Etneo, Acireale, I9Io, an. XIX, III trim., pp. I-2 du tirage à part.

Martelli, G. Principali mezzi di lotta contro gli insetti più comunemente dannosi. $R$. Scurola superiore di Agricoltura in Portici. Bollettino n. 3, serie IV, Portici, I9Io, 5I pp., $3^{8}$ fig.; $2^{2}$ ediz., Portici, I915, I02 pp., 64 fig., passim. 
MIARtins, D. Combatamos os gafanhotos! Chacaras e Quintaes, S. Panlo, Brazil, IgI I, vol. III, 11. 5, pp. 24-25, 2 fig.

MartzolfF, C. L. How we fooled the locusts. Fruit-Grower, St. Joseph, Mo., I9I6, vol. 27, 1). 239 .

Mereshkowsky, S. \$. Zur Frage der Vertilgung der Wanderheuschrecken durch Kulturen des 'Bacillus d'Hèrelle. Centralblatt für Bakteriologie, Parasitenkundc u. Infcktionskrakheitcn, Jena, I914, II. Abt., 40. Bd., No. I/8, S. I3I.

Milliken, F. B. The position assumed by female grasshoppers when ovipositing. Joumal of Economic Entomology, Concord, N. H., I9l2, vol. 5, no. 2, p. 232, fig. 3.

Milliken, F. B. C.rasshoppers and their control on sugar beets and truck crops. $L$ nited States Department of Agriculture. Farmer's Bulletin 69I, Washington, D. C., I9I5, PP. I-I6, fig. $\mathrm{I}-\mathrm{I} \mathrm{I}$.

MILlinen, R. Report on outhreacks of the western cricket and of certain locusts in Idaho. Inscet Life, Washington, I.894, vol. VI, no. I, p. I7.

Mixistère de l'Agriculture. Direction de l'Agriculture. OfFice des Renseignemexts AGricoles. Service des Études technigues [France]. Circulaite à MLM. les Professeurs départementaux et spéciaux d'agriculture en vue de prévenir, pour l'année I902, les invasions de sautérelles dans les cultures de leurs régions. Circulaire $110^{\circ} 36$. Paris, I goz, 9 pp., fig. 9.

Misisterio de Agricultura. Comisión de Defensa Agrícola [ARgentine]. Leyes nos 3708 y 4863. Decreto reglamentario. Bnenos Aires, 1906, I $7 \mathrm{pp}$.

irsisterte de Agricultura, industria, Comercio y Obras púdelicas. Dirección General DE Agricultura [Esfagne]. Memoria de la campaña contra la langosta en Igoo-Igor. Madrid, Imprentá de los Hijos de M. G. Hernández, Igol, I 78 pp., I 2 lám.

innisterio de Fomento. Dirección general de Agricultura, Minas y Montes [Fspagze]. Memoria de la campaña contra la langosta en Igro-rgl I , formada con las disposiciones dictadas por el Ministerio de Fomento, las Memorias remitidas por los Ingenieros Jefes de las Secciones agronómicas de las provincias invadidas y la cuenta general de los gastos originados en la campaña de primavera. Madrid, Imprenta "Artcs Gráficas", I9II, $301 \mathrm{pp}$.

Ministerio de Fomento. Dirección general de Agricultura, Minas y Montes [Espagne]. Memoria de la campaña contra la langosta en I9I I-I9I2, formada con las disposiciones dictadas per el Ministerio de Fonento y las Memorias remitidas por los Ingenieros Jefes de las Secciones Agronómicas de las provincias invadidas. Madrid, Imprenta "Artes Gráficas", 1913, 226 pp., 3 lám.

Ministero di Agricoltura, INDUStria E Commercto. Direzione generale dell'AgricoltUra [ITALIE]. Entonologia agraria. Manuale degli insetti nocivi alla piante coltivate, campestri, ortensi, e loro prodotti, e modo di combatterli. Redatto dalla $\mathrm{R}$. Stazione d’Entomologia agraria di Firenze. Firenze, Tip. M. Ricci, I9I5, pp. 425-443, fig. $3^{85-40 I . ~}$

IIoncada, S. El problema de la langosta y el cultivo de la alfalfa. (Traducción 'lel Fanmer's Bullatin No 637, Washington, 25 de enero de 1915). Bolctin de la Secrctaria de Fonento, Obras Públicas y Agricultur , Tegueigalpa, I9I5, t. IV, 11. IV, Pp. I95 202.

Mongrtore, A. Della Sicilia ricercata nelle cose più memorabili. Palemo, Stamperia Valenza. I 742 , lib. IX. p. 554 .

MontanaRI, MI. El nuaiz amargo en el Campo experimental de Agricultura cle la Chacarita, Revista de la A sociación Rural del Uruguay, Montevideo, I9I I, año XI, n. 10, p1). 776-775.

Montandon, A. I. Les acridiens du delta du Danube. Bullctin de la Société des Sciencés de Boncarest, I9oo, an. IX, $11^{\circ} 4, \mathrm{pP} .462-472$.

MOORE, W. The ellect of poisons upon the elegant grasshopper (Zonoccrus elegans). The Agricultural Journal of the Union of South Africa, Pretoria, r913. vol. VI, 110. I, pp. 6o-63.

Morrme, A. W. Grasshoppers. Arizona Agricultural Expcrincut Station. Timely Hints for Farmors. Ttucson, IgIt, no. Iof, I fig. 
MIorse, A. Researches on North American Acrididac. Carnegie Institution of Washington, Washington, I904, $55 \mathrm{pp}$., I2 fig., $8 \mathrm{pl}$.

IIorstatr, H. Die Wanderheuschrecken und ihre Bekämpfung. Flugblatt Nr. 7 (Beilage zum Pflanzer, Jahrg. VI.), I9I0, S. I-6 ; 2. Aufl., Daressalam, I913, S. I-7.

Morstatr, H. Die Schädlinge der Baumwolle in Deutsch-Ostafrika. Der Pflanzer, Daressalam, I9I4, Jahrg. X, Beiheft Nr. I, S. I.

Mosséri, V. Les sauterelles en Egypte. Bulletin de l'Union des Agriculteurs d'Egypte, L,e Caire, I9I 5, I $_{3}$ an., $\mathrm{n}^{\circ}$ II0, pp. 4-26.

MIoszerk. Wanderheuschrecken in Südafrika. Illustrierte landwirtschaftliche Zcitung, Schöneberg-Berlin, I899, I9. Jahrg., S. 1027-1029.

IIUnro, A. The locust plague and its suppression. London, Murray, 1900, $365 \mathrm{pp}$., fig.

Musso. Campagne d'expérimentation de la méthode biologique contre les Schistocerca peregrina, dans la région de Bougzoul- Msiline, commune mixte de Boghari (département d'Alger), mai-juin I9I5. Annales de l'Institut Pasteur, Paris, 1916, t. XXX, nº 7, pp. 319-329, fig. I-3, I carte.

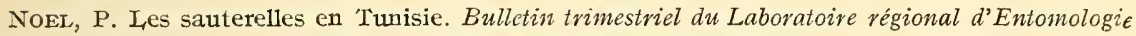
agricole de la Seine-Inférieure, Rouen, I9I6, 2 trim., pp. 3-6.

Noronha Santos, C. A destruição dos gafanhotos na Republica Argentina. Boletim do Minzsterio da Agricultura, Industria e Commercio, Rio de Janeiro, I9I2, año I, n. 4, p. 204.

ORTEL. Destruction des criquets. Système Ortel. Mustapha-Alger, I 897,28 pp., fig.

PACKARD, W. E. Control of grasshoppers in Imperial Valley. California Agricultural Expeviment Station. Circular I43, Berkeley, I9I5, II pp., 8 fg.

Packard, A. S., RILey, C. V., Thomas, C. First annual report of the U. S. Entomologica. Commission for the year I877. Relating to the Rocky Mountain locust. Department of the Interior. United States Geological Survey. Washington, D. C., I878.

PaILlor, A. Les microorganismes parasites des insectes. L,eur emploi en agriculture. Annales dut Service des Épiphyties, Paris, I9I5, t. II, pp. I 88-232, passini.*

PAOLI, G. Le cavallette in Sardegna. Cagliari, Tip. Industriale, I909, I2 pp.

PaolI, G. I,e cavallette in Sardegna. Giomale di Agricoltura della Domenica, Piacenza, I9o9, an. XIX, n. 42 , p. 356,2 fig.

PAoLI, G. Considerazioni sui mezzi di lotta sperimentati per la distruzione delle cavallette in Sardegna. Cagliari, Tipo-Iitografia Commerciale, I9ı, Io pp.

PAolI, G. Per la ricerca e la distruzione delle uova di cavallette. Cagliari Tip. e Lit. Meloni e Aitelli, I9Io, 8 pp.

PAOLI, G. Raccolta delle uova di cavallette eseguita dal febbraio all'aprile 191 o nella prov incia di Cagliari. Cagliari, Tipo-Litografia Commerciale, Iglo, ro pp.

PaolI, G. Sulla Sarcophaga lincata Fallen parassita dello Stauronotus maroccanus (Thunb.) in Sardegna. Bollettino del Laboratorio di Zoologia generale e agraria della R. Scuola superior, d'A Aricoltura in Portici, Portici, I910, vol. IV, pp. 347-352.

Pereyra, A. La langosta. Historia, costumbres y medios de destrucción en la República Argentina y otros países. Buenos Aires, M. Rodriguez Giles, I909, pp. V- 293, 27 grabados, II mapas.

PEтch, C. E. Insects injurious in Southern Quebec, I9I4. Forty-Fifth Annual Report of the Entomological Society of Ontario, I9I4. Toronto, I9I5, p. 7I.

PETrit, R. H. Insects of the garden. Michigan State A gricultural College Experiment Station. Department of Entomology. Bulietin 233, Agricultural College, Michigan, I905, pp. 55-57, fig. $56-5 \%$.

PETrit, R. H. Insects of fields crops. Michigan State Agricultural College Experiment Station. Division of Entomology. Bulletin 258, Agricultural College, Michigan, r910, pp. 64-65, fig. $32-33$.

PICARD, F. et BLANC, G.-R. Les infections à coccobacilles chez les insectes. Comptes rendus hebdomadaires des séances de l'A cademie des Sciences, Paris, I9I3, t. I57, nº I, pp. 79-8I. 
Pratt, H. C. General notes concerning locusts. The Agricultural Bulletin of the Federated Malay States, Singapore, I9r3, vol. I, no. r2, pp. 428-430.

PRATT, H. C. The locust pest in Malaya. A short survey and a brief description of its lifehistory. The Agricultural Bulletin of the Federated Malay States, Singapore, I9I3, vol. II, no. 4 , pp. $76-80$.

PRATT, H. C. Locust spraying experiments. The Agricultural Bulletin of the Federated Malay States, Singapore, I9I 4, vol. II, no. IO, pp. 249-255.

Pratt, H. C. and DE LA Mare Norris, F. The Malayan locust (Pachytylus sp.) with notes on distribution and methods of control. Department of Agriculture, Federated Malay States. Bulletin no. 24, Singapore, I9I5, 42 pp., 4 fig., I6 pl., I map.

Pratt, H. C. and South, F. W. Progress report on locust work since june 19I3. The Agricultural Bulletin of the Federated Malay States, Singapore, I9I3, vol. II, no. 3, pp. 53-59.

Pratt, H. C. and South, F. W. Progress report on locust work to november 30 th I9I3. The Agricultural Bulletin of the Federated Malay States, Singapore, I9I4, vol. II, no. 6 , pp. I52-I56.

RABAUD, E. Sur quelques réflexes des orthoptères acridiens. Comptes rendus hebdomadaires des séances de la Société de Biologie, Paris, I9I5, t. LXXVIII, no I9, pp. 668-67I.

Ravojević, $N$. Die in Serbien in den Jahren r906-1909 beobachteten Pflanzenkrankheiten und Schädlinge. Zeitschrift für Pflanzenkrankheiten, Stuttgart, I9II, XXI. Bd., Jahrg. I9II, S. 46 .

REH, L. Phytopathologische Zoologie für unsere Kolonien. Der Tropenpflanzer, Berlin, Igri, I5. Jahrg., Nr. 3, S. I 44 .

ReH, I. Die tierischen Feinde. Dans Sorauer, P. Handbuch der Pflanzenkrankheiten. 3. Aufl. Berlin, P. Parey, I9I3, III. Bत., S. I50-212, Fig. I22-15I*.

Repartição de Agricultura. Secção de Entomologia [Mozambigue]. Antidotos contra o envenenamento produzido pelo arsenico e seus preparados. Lonrenço Marques, I 909.

Repartição de Agricultura. SeCção de Entonologia [MozambiQue]. Instrucções pata o uso do "Locusticida " para a destruição dos gafanhotos. Lourenço Marques, I 909.

República Argentina. Dirección General de Defensa Agrícola. Instrucciones pata la destrucción de la langosta. Buenos Aires, I910, 60 pp., 24 fig.

República Oriental del Uruguay. Defensa agrícola. La langosta. Publicación no ${ }^{\circ}$, Montevideo, Talleres Gráficos A. Barreiro y Ramos, I9I2, 39 pp., I 4 fig.

República Oriental del Uruguay. Defensa Agrícola. Ley y reglamentación. Exhortación a los hacendados y agricultores del país. Montevideo, Talleres Gráficos A. Barreiro y Ramos, I9i2, 36 pp., passim.

República Oriental del Uruguay. Defensa agrícola. Principales enemigos de nuestros cultivos y medios para combatirlos. Publicación $n^{\circ}$. 4, Montevideo, Talleres Gráficos A. Barreiro y Ramos, I9I3, p. 9.

República Oriental del Uruguay. Ministerio de Industrias. Defensa Agrícola. Ia langosta. Conocimientos útiles para el caso de una invasión del acridio. Publicación $n^{0} .5$, Montevideo, Talleres: La Razon, el Siglo y Telégrafo, I9I4, 8 pp., I fig.

República Oriental del Uruguay. Ministerio de Industrias. Defensa Agrícola. Memoria correspondiente al año I914. Montevideo, Talleres Gráficos del Estado, 1915, pp. 24-36, 4 fig.

República Oriental del Uruguay. Ministerio de Relaciones Exteriores. Conferencia internacional de Defensa Agricola, de Montevideo (Mayo de I9I3). Texto de las Convenciones sancionadas. Montevideo, Talleres Gráficos A. Barreiro y Ramos, I9I3, pp. 5-го.

Résidence générale de la République Française au Maroc. Agriculture. Notice sut les moyens de défense contre les sauterelles, I9 $5, \mathrm{n}^{0} 5$, I $4 \mathrm{pp}$. (éd. franç.); $20 \mathrm{pp}$. (en langue arabe).

RICKMANy and KAESEWURm. Beobachtungen über Entwikelung und Verwendung des Heu- 
schreckenpilzes in Deutsch-Südwestafrika. Notizblatt des königl. botanischen Gartens und Museums, Berlin, Leipzig, I900, Nr. 24, pp. 65-74.

Rivas Moreno. Los estragos de la plaga de langosta. Resumen de Agricultura, Barcelona, I9I6, año XXVIII, cuaderno $\%$, pp. 295-300.

Rivas Moreno. Los viñedos, los patatares y la langosta. La Vinicultura Española, Madrid, I9I6, año VI, núm. I26 [p. I].

Rtvas Moreno. I,a plaga de langosta y los cerdos. Resumen de Agricultura, Barcelona, I9I6, año XXVIII, cuaderno Io, pp. $46 \mathrm{I}-462$.

Rivik̀re, CH. Sauterelles et criquets. Bulletin de la Société Nationale d'Acclimatation de France, Paris, r9I4, 6I e an., $n^{\circ}$ I 6, pp. 535.540.

RIVIÈRE, CH. [Les sauterelles]. Bulletin de la Société Nationale d'Acclinatation de France, Paris, I9I 6,63 e an., no 3 , pp. Ior-Io2.

RIvière, Ch. et LEcQ, H. Traité pratique d'Agriculture pour le Nord de l'Afrique. Paris, A. Challamel, I9I 4, pp. 808-8I $6^{*}$.

Rodríguez Martín, R. Nuevas aplicaciones con el ácido cianhidrico, para la extinción de la plaga de langosta y el pulgón de la vid. (Altica ampelophaga). (Fxperiencias en el laboratorio y en el campo). Toledo, Establecimiento tipográfico de Rafael Gómez-Menor, I9I2. 44 pp., 4 lám.

RoEpke, W. Verslag over het jaar I9I4-I9I5 en Verkprogramma voor het jaar I9I5-I9I6. Mededeelingen Proefstation Midden Java, Batavia, I9I 5, No. 20, $30 \mathrm{blz}$.

Roepke, W. Sprinkhanenplagen. Teysmannia, Batavia, I9 I5, 26 ste jaarg., I en $2^{e}$ aflev., blz. I I5-I24; $6^{\mathrm{e}}$ en $7^{\mathrm{e}}$ aflev., blz. 337-358, I fig., pl. I-V ; I2 e aflev., blz. $758-590$, I fig., p1. VI-VII*.

RORER, J. B. Report on the inoculation of locusts with Coccobacillus acridiorum. Bulletin of the Department of Agriculture, Trinidad and Tobago, Port-of-Spain, x9r5, vol. XIT, part 6, pp. I97-I98.

Rutgers, A. A. I. Ziekten en plagen der Cultuurgewassen in Nederlandsch-Indië in I 9 I 4. Departement van Landbouw, Nịverlueid en Handel. Instituut voor Plantenziekten en Cultures. Mededeelingen van het Laboratorium voor Plantenziekten, Batavia, I9I5, No. I5, blz. I.t-I5, I 8-r 9,33 .

SA Jó, K. A marokkói sáska (Stauronotus maroccanus Thunb.). Magyarországon az r 888 , I 889 , és I 890 , években. Budapest, I 89 r, 79 lap, 8 ábra.

SAJó, K. Jelentés az I 884 -I 889 években felmerült gazdasági rovarkárakról. Budapest, I 892. (Bericht über die in Ungarn in den Jahren I 884-I 889 vorgekommenen landwirtschaftlichen Insektenschäden). Zeitsch"ift für Pflanzenkrankheiten, Stuttgart, I894, IV. Bd., S. I52.

SAJó, K. Bericht über die in den letzten Jahren in Ungarn aufgetretenen Insektenschäđen. Zeitschrift für Pflanzenkrankheiten, Stuttgart, I895, V. Bd., S. 36I-363.

SANDER, I. Die Wanderheuschrecken und ihre Bekämpfung in unseren afrikanischen Kolonien. Berlin, Dietrich Reimer, I902, passim.

SANDERson, E. Dwight. Report on miscellaneous cotton inserts in Texas. U. S. Department of Agriculture. Bureau of Entomology. Bulletin no. 57, Washington, r9o6, pp. I9-26, fig. 8-I3.

SANDERSON, F. DWrGht. Insect pests of farm, garden and orchard. First edition. New York, John Wiley and Sons, I9I2, pp. 93-ז13, fig. 62-82.

Savastano, I. La pompa n. 6 per la poltiglia solfo-calcica e la 11 . 7 ler il petrolio benzinato contro le cavallette. $R$. Stazione sperimentale di Agrumicoltura e Frutticoltura, Acireale. Bollettino n. 24, Acireale, I9r6, pp. 2-3, fig. 2.

Schenk, I. Madaraktól meghiúsított sáskajárás. Von der Vogelwelt verhinderte Heuschreckenplage. Aquila, Budapest, Igro, XVII évfol. (XVII. Jahrg.), S. 258-26I.

Schouteden, H. L,es orthoptères nuisibles aux plantations en Afrique. Rcrue Zool. Afric., Bruxelles, I9I 4, vol. III, no 3 , pp. 464-470, 2 p1.

Schnürer, J. und Rohonyi, N. Zur Kenntnis des Kokkobazillıs d'Hérelle, eines Heuschrecken- 
schädlings. Deutsche ticrärztliche Wochenschritt, Hannover, I9I4, 22. Jahrg., Xr. 42, S. $601-603$

Schroner, J. Versuche zur Bekämpfung der Wanderheuschrecke mit chemischen Produkten. Zeitschrift für Pflanzenkrankheiten, Stuttgart, I909, XIX. Bd., Heft I, S. I-I3, Fig. I-4. Scrroder, J. Beitrag zur Kenntnis der chemischen Zusammensetzung der Wanderhcuschrecke, ihrer Eier und der noch ungeflügelten Brut. Zeitschrift für Pflanzenkrankheiten, Stuttgart, I go9, XIX. Bd., Heft I, S. I3-18, Taf. I.

Shroff, K. D. Cotton pests in Burma. Department of Agriculture, Burma. Bulletin no. 8, I9I2, Rangoon, I9r3, pp. 25-26, pl. XI.

Secretaría de Fomento. Commsión de Parasitología Agrícola [Mexique]. La langosta y la agricultura. Circular núm. 74 , México, Imprenta y Fototipía de la Secretaría de Fomento, I907, 24 pp., 26 fig.

Secretaría de Fomento. Esstación Agrícola Experimental de Ciudad Juárez, Chindahua. Tres plagas de la agricultura : El chapulín o langosta (Acrididae). - El pulgón del durazno (Aphis persicae). - El gusano del álamo (Hyphantria cunea). Boletín núm. 5, México, 1906, DP. 5-II, I fig.

Secretaria Geral do Governo da Provincia de Moçamibique. Repartição de, Agricultera. Secção de Entomologia. Methodos a empregar para a destruição dos gafanhotos. Circular II, Lourenço Marques, Imprensa Nacional, Igo8, $8 \mathrm{pp.}$

SERGent, EDm. Rapport sur le fonctionnement de l'Institut Pasteur d'Algérie $\in$ n I9I 5. Alger, Imprimerie administrative E. Pfister, I916, passim.

Sergent, Edm. el LHÉrrtmer, A. Essai de destruction des sauterelles en Algérie par le "Coccobacillus acridiorum " de d'Hérelle. Annales de l'Institut Pasteur, Paris, I9I4, t. XXVIII, $\mathbf{n}^{\circ} 4$, pp. 408-4r9, 2 fig.

Sergent, FDM. et LHÉritrer, A. Essai de destruction des sauterelles en Algérie par le "Coccobacillus acridiorum " de d'Hérelle. Bulletin de la Société Nationale d'Acclimatation de France, Paris, I9I4, IXI an., $n^{\circ}$ I 4 , pp. 456-467.

SERGENT, ÉT. Campagne d'expérimentation de la méthode biologique contre les Schistocerca peregrina dans la vallée de la Haute Tafna, commune mixte de Sebdou (départment d'Oran). Existence d'une épizootie autochtone vaccinante (mai, juin, juillet I9I5). Amnales de l'Institut Pasteur, Paris, I916, t. XXX, no 5, pp. 209-224, fig. I-10.

SERre, P. A. I'île de la Trinité menacée d'une invasion de sauterelles. Comptes rendus des séances de l'A cadémie d'Agricr.lture de France, Paris, I9I6, t. II, no Io, pp. 347-352.

Settler, A. Re Locusts. Nairobi News, Nairoli, I 905 , vol. I, no. I, p. 2.

SHaRP, D. Insecta.Dans The Cambridge Natural History, London, Macmillan and Co., Ltd., I910, vol. V, pp. 279-329, fig. I65-203, passim*.

Sm тH, H. E. The grasshopper outbreak in New Mexico during the summer of I9I3. United States Department of Agriculture. Professional Paper. Bulletin 293, Washington. D. C., I9I5, I 2 pp., 2 fig.*

Somes, M. P. The Acridiidae of Minnesota. University of Minnesota. Agricultural Experiment Station. Technical Bulletin I4I, St. Paul, I9I4, Ioo pp., II fig., 4 pl.

South, F. W. Work on locust destruction in september. The Agricultural Bulletin of the Federated Malay States, Singapore, 19I3; vol. II, no. 4, pp. $85-88$.

Soutr, F. W. The agricultural pests enactment no. 13 of I9I3. The Agricultural Bulletin of the Federated Malay States, Singapore, I9I4, vol. II, no. 9, p. 222.

South, F. W. Report on the work of locust destruction january Ist to march I5, I 9 I 4 The Agricultural Bulletin of the Federated Malay Statcs, Singapore, IcI 4, ro1. II, 10. 9 pp. $227-230$.

South F. W. Summary of locust work march 12 to april 30, I9I4. Selangor. The Agricultural Bulletin of the Federated Malay States, Singapore, I9I 4, vol. II, no. II, pp. 294-297.

Souxr, F. W. Report on locust work may ist to july 5th. The Agricultural Bulletin of the Federated Malay States, Singapore, I9I4, vol. II, no. I2, pp. 323-326. 
South, F.W.A summary of locust work in I9I4. The Agricultural Bullctin of the Fcderated Malay States, Singapore, I9I5, vol. III, no. 8, pp. 293-297.

Soutr, F. W. The enactment to amend "The agricultural pests enactnieni, I9I3". The Agricultural Bullctin of the Federated Malay States, Singapore, I9I5, vol. III, no. 8 pp. $297-299$.

South, F. W. Summary of locust work for the third quarter, july to september, I9r5. The Agricultural Bulletin of the Federated Malay States, Singapore, I9I5, vol. IV, no. 3, pp. $68-72$.

South, F. W. Summary of the locust work for the $4_{\text {th }}$ quarter, I9I5. The Agricultural Bulletin of the Federated Malay States, Singapore, I9I6, vol. IV, no. 5, pp. I42-I45.

South, F. W. A summary of locust work in I9I5. The Agricultural Bulletin of the Federated Malay States, singapore, I9I6, vol. IV, no. 5, pp. I 46 -I 5 0.

South, F. W. Summary of the locust work for the ist quarter, I9I6. The Agricultural Bulletiv of the Federated Malay States, Singapore, x9I6, vol. IV, no. 9, p1. 29I-297.

South, F. W. and DE lA MARe Norris, F. Progress report on locust work for the months of july, august and september, r9r4. The Agricultural Bulletin of the Federated Malay States, Singapore, I9I5, vol. III, no. 4, pp. I55-I57.

STOREy, G. List of Egyptian insects in the collection of the Ministry of Agriculture. Ministry of Agriculture. Technical and Scientific Service. Bulletin no. 5 (Entomological Section), Cairo, I9I6, pp. 2-4.

Summers, W. I. Destruction of locusts. The Journal of the Department of Agriculture of South A ustralie, Adelaide, r909, vol. XIII, no. 4, pp. 297-30I, fig. 5-9.

TARgioni Tozzettr, AD. Ortotteri agrari, cioè dei diversi inselti dell'ordine degli ortotteri, nocivi o vanłaggiosiall'agricoltura e principalmeute delle carallette. Ministero di Agricoltura, Industria e Commercio. Direzione dell'Agricoltura. Annali di Agricoltura, I882, FirenzeRoma, Tip. dei fratulli Bencini, 1882 , passim*.

Targioni Tozzeti, Ad. Distruzioue delle cavallette. R. Prefettura della prov. di Firenze, I 896 , div. $4^{\mathbf{a}}, \mathrm{n}^{\mathrm{o}} 389 \mathrm{I}$.

TAvares, J. S. A praga dos gafanhotos na Republica Argentina. Broterta, Serie de vulgarização scientifica, Braga, I9I2, vol. X, fasc. VI, pp. 404-405.

TAYLOR, I. E. Notes on birds likely to be of service in the desiruction of grasshoppers in the Nicola Valley. Proceedings of the Entomological Society, Victoria, British Columbia, I9I5. no. 7 , pp. $43-45$.

Téllez Pizarro, M. Ira plaga de la langosta. Secretaria de Fomento. Comisión de Parasitología A gricola. Circular núm. 52, México, I906, pp. I-I I, fig. I-II.

Iennant, H. F. Invasion of locusts in Salvador. Daily Consular and Trade Reports, Washington, I9I 4, I $7^{\text {th }}$ year, no. 246 , p. 349 .

Thomas, C. Manual of Economic Entomology. Part III: The Acridiidae of Illinois. Ninth Report of the State Entomologist, I880, vol. III, pp. 73-I40.

Thomsen, F. The brown locust campaign, I908-09. The Transwaal A gricultural Joninal, Pretoria, I909, vol. VII, no. 27 , pp. 52x-529, pl. 8 I-83.

Thomsen, F. The redwing locust campaign, season $1908-09$. The Transwaal Agricultural Journal, Pretoria, I909, vol. VII, no. 28, pp. 678-679.

Tromsen, F. Locust birds. The A gricultural Journal of the Union of South Africa, Pretoria. I9I4, vol. VII, no. 5, pp. $682-683$.

TORRE, E. Le cavallette nell'Italia meridionale. Calamità ricorrente. Mezzi di lotta. Giomale dí Agricoltura della Domenica, Piacenza, I9I3, an. XXIII, n. 30, pp. 236-237, 9 fig.

T.[RABUt]. Ia lutte contre les sauterelles. Bulletin Agricole de l'Algérie-Tunisie-Maroc, Alger, I 9 I $5,2^{\mathrm{e}}$ série, $2 \mathrm{I}^{\mathrm{e}}$ an., to 3 , pp. 53-74, I 6 fig.

Trabut, L. Les champignons parasites du criquet pèlerin. Revne générale de Botanique, Paris, I 89r, vol. III, pp. 40r-405, I pl. 
Trabut, L. Les sauterelles en I9I6. Revue Horticole de l'Algérie, Alger, I916, t. XX, 20e an., pos I-2, pp. 34-36; Bulletin Agricole de l'Algérie-Tunisie-Maroc, Alger, I9I5 [1916], $2^{\mathrm{e}}$ série, $21^{\mathrm{e}}$ an., $\mathrm{n}^{0}$ I $2,1 \mathrm{p}$. 308-3xr.

Travers, W. L. Locusts in North Bengal. Journal of the Bombay Natural History Soeiety, Bombay, I9I5, vol. XXIV, no. ז, pp. I97-I98.

Treat, M. Injurious insects of the farm and garden. New York, Orange Judd Company, I9o8, pp. $269-282$, fig. $158-163$.

Trotter, A. Malattie e parassiti delle pionte coltivate. Dans Ministero di Agricoltura, INDUSTRIA E Commercio. Ricerche e studi agrologici sulla Líbia. Bergamo, Istituto italiano d'Arti grafiche, I9I2, vol. I : La zona di Tripoli, p. 379.

Tryon, H. Grasshopper destruction and white ants. The Queensland A gricultural Journal, Brisbane, I9o3, no. 3 , pp. $282-285$.

Tyler Townsend, C. H. On the injurious and other locusts of New Mexico and Arizona. Insect Life, Washington, I894, vol. VI, no. I, p. 29.

Union of South Africa. Department of Agriculture. Division of Entomology. Locust destruction. No. 75. Pretoria, I9I5, pp. 3-8.

URICH, F. W. Annual report of the entomologist. Port-of-Spain, IgIo, p. 5.

URICH, F. IV. Locusts and methods of destroying them. Board of Agriculture, Trinidad and Tobago. Circular no. I3, Port-of-Spain, I9I5, $6 \mathrm{pp}$.

URICH, F. W. I,ocusts or grasshoppers. Bulletin of the Department of Agriculture, Trinidad and Tobago, Port-of-Spain, I9I5, vol. XIV, part 4, pp. r20-I28, I map.

URICH, F. W. Insects affecting the sugar cane in Trinidad. Bulletin of the Department of Agriculture, Trinidad and Tobago, Port-of-Spain, 1915, vol. XIV, part 5, p. I60.

URICr, F. W. Insects affecting the coconut palm in Trinidad. Bulletin of the Department of Agriculture, Trinidad and Tobago, Port-of-Spain, I9I5, vo1. XIV, part 6, p. 203.

URICH, F. W. Notes on the South American locusts, Schistocerca paranensis, Burm. Bulletin of the Department of Agriculture, Trinidad and Tobago, Port-of-Spain, I9I6, vol. XV, part I, pp. $15-16$.

VAN HALL, C. J. J. Ziekten en plagen der Cultuurgewassen in Nederlandsch-Indië in I9I5. Departement van Landbouw, Nijverheid en Hanael. Instituut voor Plantenziekten en Cultures. Mededeelingen van het Laboratorium voor Planienziekten, No. 20, Batavia, I9ı6, blz. 3-47, passim.

VAN Ryneveld, A. Locust destruction, I909-I9ro. The A gricultural Journal of the Cape of Good Hope, Cape Town, I9Io, vol. XXXVI, no. 2, pp. I58-I66.

VARgetto, G. Le cavallette. L'Ora, Palermo, I9Io, an. XI, n. I 10.

VELU, H. et BouIN, A. Essais de destruction de "Schistocerca peregrina " Olivier au Maroc, par l'emploi des cultures microbiennes ( Coccobacillus acridiorum » d'Hérelle). Bulletin de la Société de Pathologie exotique, Paris, x9I5, t. VIII, no 9, pp. 638-64I.

Velu, H. et Bourn, A. Essai de destruction du Sehistocerca peregrina au Maroc par le "Coccobacilius aciidiorum " du $\mathrm{D}^{r}$ d'Hérelle. Annales de l'Institut Pasteur, Paris, I9I6, t. XX. n० 8, pp. $389-42$ r, 7 fig.

von IHERTNG, H. A patria das nuvens de gafanhotos. Chacaras e Quintaes, S. Paulo, Brazil, I9II, vol. III, n. 5, pp. 2 I-23, 3 fig.

von Ihering, R. Diccionario da fauna do Brazil. Almanak Agricola Brazileiro, S. Paulo, I9r3, pp. 280, 305 .

VON ZELLES, A. Die Heuschreckengefahr in Ungarn. Österreichisches landwirtschaftliehes Wochenblatt, Wien, I904, 30. Jahrg., S. I46-14\%.

Vosseler. Die Wanderheuschrecken in Usambara im Jahre I903-I904, zugleich eir Beitrag zu ihrer Biologie. Berichte über Land-und Forstwirtschaft in Deutseh-Ostafrika, Heidelberg, I905, II. Bd., Heft 6, S. 291-374, Fig. I-2, Taf. XII-XIII.*

Vosseler, J. Neues über den Heuschreckenpilz. Der Pjlanzer, Tanga, Igod, Bd. 4, S. $I \succ I-I 73$. 
WAHL, B. Die biologische Metlode der Bekämpfung von Pflanzenschädlingen. Sonderabdruck aus: Verhandlungen der vierten Tagung und der Hauptversammlung der Osterr.-und Pomologen Gesellschaft. Wien, I9I4. Im Selbstrerlag. Druck von Friedrich Sper1. S. 6.

WALkER, E. M. The Orthoptera of Western Canada. The Canadian Entomologist, rgro, vol. 42 , pp. $269-276,293-300,333-3+0,35$ I-356.

WALKER, E. M. Notes on a collection of Orthoptera from Prince Edwırd Island and the Magdalen Islands, Que. The Canadian Entomologist, London, I9I5, vol. XITII, no. Io, pp. 339-344.

WALKER, E. M. Popular and practical entomology. A few days in Newfoundland. The Canadian Entomologist, London, I9I6, vol. XI,VIII, no. 7, p. 221.

WASHBURN, F. L. Cutworms, army worms and grasshoppers. University of Minnesota. Agricultural Experiment Station. Division of Entomology. Bulletin no. I23, St. Paul, Minn., pp. $73-84$, fig. I $7-26$.

WASHBURN, F. I. Grasshopper work in Minnesota during the season of I 9 I r. Journal of Economic Entomology, Concord, N. H., I9I2, vol. 5, no. 2, pp. III-I2I.

Webster, F. M. The grasshopper problem and alfalfa culture. U. S. Department of Agriculture. Bureau of Entomology. Circular 84, Washington, I90\%, Io pp., 8 fig.

IVEBster, F. M. Some developments in grasshopper control. Journal of Economic Entomology, Concord, N. H., I9I5, vol. 8 , no. 6 , pp. 527-535, fig. 2 I.

Webster, F. M. The grasshopper problem and alfalfa culture. U. S Department of Agriculture. Farmers' Bulletin 63\%, Washington, I9I5, Io pp., 8 fig.

WEBSTER, F. M. Recent grasshopper outbreaks and latest methods of controlling them. Yearbook of the United States Department of Agriculture, I9I5, Washington, I9I6, pp. 263-272, fig. $7-9$, pl. I,I-IVI.

WrLlis, J. C. Visitation of spotted locusts. Circulars, Royal Botanic Gardens, Ceylon, Colombo, I 898 , ser. I, no. 9, pp. $77-8$ I.

IVILson, H. F. Grasshoppers in Oregon. Oregon Agricultural College Experiment Station. Second Biennal Crop Pest and Horticultural Report, I9I3-I9I4, Corvallis, Oregon, I9I5, pp. I33-I36, fig. 26-27.

WILson, T. The outbreak of locusts in rgr4. Proceedings of the Entomological Society, Victoria, British Colombia, I 9 I 5 , no. 7, pp. 4I-43.

Woodworth, C. IV. Grasshoppers in California. University of California Publications. College of Agriculture. Agricultural Experiment Station. Bulletin no. I42, Sacramento, I9o2, 36 pp., I8 fig.

X. Pensiamo a difenderci dalle cavallette. Gazzetta Commerciale, Palermo, I9Io, an. XI, n. 666 et $66 \%$.

ZACHER, FR. Die wichtigsten Krankheiten und Schädlinge der tropischen Kulturpflanzen und ihre Bekämpfung. Hamburg, Fr. W. Thaden, I9r4, I. Bd., S. 36-38, Abb. 9.

ZANGHI, P. Sulle cavallette e del modo di distruggerle. $2^{\text {a }}$ ediz. Messina, Giuseppe Fiumara, I $840,393 \mathrm{pp}$.

ZAPPELli, P. Contro un insetto dannoso. L'Agricoltura Sabina, Poggio Mirteto, I9I4, vol. XIII, n. 4 , p. I 8 .

ZIMMERVANN, H. Über das Massenauftreten namentlich schädigender Insektenformen. Zeitschrift für Pflanzenkrankheiten, Stuttgart, I9I I, XXI. Bd., S. 266.

Agricultural defense. Bulletin of the Pan American Union, Washington, D. C., U. S. A., I9Io, pp. $680-681$.

Argentine plains and Andine glaciers. With a description of the South American locust. Bulletin of the Pan Anerican Union, Washington, D. C., U. S. A., I9I I, pp. Io82-I094, I6 fig.

Bekämpfung (Die) der Heuschrecken. Der Tropenpflanzen, Berlin, rgoo, 4. Jahrg., S. 87-93. 
Bekämpfung (Die) der Heuschrecken in Dalmatien. Wiener landwirlschaftliche Zeitung, Wien, I912, 62. Jahrg., Nr. 56 (Nr. 4752), S. 666.

Campaña (La) contra la langosta. República Argentina. Boletín del Ministerio de Agricultura, Buenos Aires, I9I4, tomo XVIr, núm. 2, p. I59.

Conferencia internacional de Defensa Agrícola. República Oriental del Uruguay. Rerista del Ministerio de Industrias, Montevideo, I913, n. ${ }^{\circ}$ I, pp. 77-84, I fig.

Contra la langosta. Acción conjuncto del Uruguay y Brasil. El Estanciero, Montevideo, I916, año VI, n. ${ }^{\circ}$ I34, p. I3.

Convención I. de Defensa Agrícola. Paises adherentes. El Estanciero, Montevideo, 19I6, año VI, n. ${ }^{\circ}{ }^{2} 32$, p. Ir.

Defensa internacional contra la langosta. Revista de la Asociación Rural del Uruguay, Montevideo, I9II, año XI, núm. 5, pp. 38I-382.

Défense des cultures. Bulletin agricole de l'Algérie-Tunisie-Maroc, Alger, $19 \mathrm{I} 5,2^{\mathrm{e}}$ série, $2 \mathrm{I}^{\mathrm{e}}$ an., no $\mathrm{n}, \mathrm{pp} . \mathrm{I}-4$.

Destrucción de la langosta efectuada en el mes de mayo de I9I2 y resumen de lo destruido en la campaña actual. Boletin del Ministerio de Agricultura, Buenos Aires, I912, tomo XIV, núm. 6, p. 527.

Destrucción ( $\mathrm{L}_{\mathrm{a}}$ ) de la langosta. Estudios y observaciones. Gaceta Rural, Mercantil é Ináustrial, Buenos Aires, I91 I, año V, núm. 5I, p. 178 .

Destrucción de plagas. Boletín del Ministerio de Agricultura, Buenos Aires, I912, tomo XIV, núms. II y I2, pp. I448-I 449 ; I9I3, tomo XVI, núm. I, p. 4 ; núms. 2 y 3, p. 226, 364 ; núm. 4 , pp. 420 ; núms. 5 y 6, pp. 533-534, 7r7 ; I9I4, tomo XVII, núm. I, pp. 9-Io ; núm. 2, p. I6o ; núms. 3 y 4, pp. 299, 440 ; núm. 5 , p. 49 ; nún. 6, p. 643 ; tomo XiVII, núms. I y 2, pp. 3 , I72 ; núms. $3,4,5$ y 6, pp. 207,348 ; 1915, tomo XIX, núms. I-2, p. 3 ; núms. $3-4$, pp. I4I-I 43 ; núms. $5-6-7$, p. 336 ; núms. $8-9$, P. 524 ; núms. IO-YI-I2, pp. 735-736; 1916, tomo XX, núms. I y 2 , p. 36 .

Destruction des criquets par le procédé Ortel. S. d., 7 pp., 7 fig.

Destruction des sauterelles au moyen du son empoisonné. Le Journal d' Agriculture et d'Horticulture illustré, Québec, I9I5, vol. I9, no. I, p. 2; no. 3, p. 50.

Destruction of grasshoppers. The Queensland Agricultural Journal, Brisbane, I9I 2, rol. XXVIII, part 6, pp. 450-452.

Destruição de gafanhotos. Boletim da Agricultura, Campinas, S. Paulo, 1906, vol. 7, n. 2, pp. $67-68$.

Elegant (The) grasshopper. The Agricultural Journal of the Union of South Africa, Pretoria, r912, vol. IV, no. 4, pp. 753-755.

Estado de la langosta. Boletín del Ministerio de Agricultura, Buenos Aires, I9I2, tomo XIV, núm. 9 , pp. $848-849$; 19r3, tomo XV, núm. 3, p. 259 ; tomo XVI, núms. 5 y 6, pp. 533, 715 ; I9I $_{4}$, tomo XVII, núm. I, p. 8.

Gafanhotos. Boletim da Repartição de Agricultura, Lourenço Marques, I9I4, 110.s I6-I8, pp. $175-180$.

Gases (L,os) asfixiantes en la destrucción de la langosta. Gaceta Rural, Buenoミ Aires, rgI6, año IX, no. 105, p. 543 .

Grasshopper baits. Extra oranges or lemons added to the Criddle mixture or to poison bran bait add to effectiveness. U.S. Department of Agriculture. Weekly News Letter, Washington, D. C., I9I 5, vol. III, no. 5 , p. 3 .

Grasshopper (The) in Queensland, The Queensland Agricultural Journal, Brisbane, I909. vol. XXII, p. 278, pl. XXXVI.

Grasshoppers destruction in South Africa. The Quecnsland Agricultural Journal, Brisbane, I 909, vol. XXII, pp. $276-278$.

Hougo (El) destructor de los acridios ó langostas. Boletin de la Comisión de Parasitología Agricola, Mexico, I902, vol. I, pp. 318-336, 5 lám. 
Insectos que atacan la caña de azúcar. Parte II. La Hacienda, Buffalo, N. Y., E. U. A., I9I6, vol. XI, núm. VIII, p. 243 , I fig.

Invasão (A) de gafanhotos. Boletim da Associação Central da Agricultura Portuguesa, Lisbõa, r9r6, ano XVIII, vol. XVIII, n. ${ }^{\circ}$, pp. 259-260.

Invasion ( $\left.\mathrm{I}_{1}^{\prime}\right)$ des sauterelles. Bulletin de l'Union des Agriculteurs d'Egypte, Le Caire, I9r5, I ${ }^{\mathrm{e}}$ an., $\mathbf{n}^{\circ}$ III, pp. $72-74$.

Invasione di cavallette in Tunisia. Giornale di Agricoltura della Domenica, Piacenza, I9r5, an. XXV, n. 25, p. 202.

I.angosta. Boletín del Ministerio de Agricultura, Buenos Aires, I9I5, tomo XIX, núms. 8-9, p. 525 .

Iangosta. Destrucción efectuada en el mes de junio y resumen de la misma desde el $\mathrm{r}^{\circ}$ de abril al 30 de junio de r9r2. Boletín del Ministerio de Agricultura, Buenos Aires, I9I2, tomo XIV, núm. 7, p. 553 .

Langosta. Invasiones y movimiento de mangas en el mes de septiembre de i9r2. Boletin del Ministerio de Agricultura, Buenos Aires, I9I2, tomo XIV, núm. Io, pp. I05I-I054.

Langosta (La). Boletin de Fomento, San José, Costa Rica, I9I3, vol. III, n. I I, pp. 830-83I.

Langosta (La). El Estanciero, Montevideo, I9I6, año VI, n. I3o, pp. 9-II.

Langosta (I,a): (Schistoccrca paranensis). La Propaganda, Montevideo, I9r4, año XIII, n. 300 ,

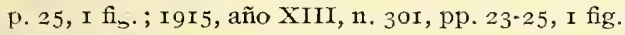

Irangosta (I,a) en agosto. Boletín del Ministerio de Agricultura, Buenos Aires, I9I3, tomo XVI, núms. 2 у $3, \mathrm{pp}$. 359-362.

Langosta (La) en Bolivia. Boletin del Ministerio de Agricultura, Buenos Aires, I9I2, tomo XIV, núm. 6, p. 525 .

Langosta $(\mathbf{I}, \mathrm{a})$ en diciembre. Boletin del Ministerio de Agricultura, Buenos Aires, I913, tomo XVI, núm. I, pp. 35-36.

Langosta (La) en julio. Boletin del Ministerio de Agricultura, Buenos Aires, I9I2, tomo XIV, núm. 8, p. 647 .

Iangosta (La) en junio. Boletin del Ministerio de Agricultura, Buenos Aires, r9r2, tomo XIV, núm. 7, p. 55 I.

Langosta (I,a) en junio. Boletin del Ministerio de Agricultura, Buenos Aires, I9I3, tomo XVI, núm. I, p. r.

Iangosta (L,a) en mayo. Boletín del Ministerio de Agricultura, Buenos Aires, I9r2, tomo XIV, núm. 6 , p. 525 .

Langosta (I,a) en mayo. Boletin del Ministerio de Agricultura, Buenos Aires, I9I3, tomo XV, núm. 6, pp. 6r6-6r 7 .

Langosta (I,a) en noviembre. Boletin del Ministerio de Agricultura, Buenos Aires, I9I2, tomo $\mathrm{XIV}$, núms. II y I2, pp. I446-I 447.

I,angosta (La) en octubre. Boletín del Ministerio de Agricultuna, Buenos Aires, I916, tomo XX, núms, I y 2, p. 37 .

Langosta (La) en septiembre. Boletin del Ministerio de Agricultura, Buenos Aires, r9r3, tomo XVI, núm. 4, p. 4 I9.

Langosta (L,a) tucura. Revista de la Asociación Rural del Uruguay, Montevideo, I9I 2, aũo XI,I, n. ${ }^{\circ}$ Io, pp. $785-786$.

Langosta (Ira) tucura en el Río Negto. Boletin del Ministcrio de Agricultura, Buenos Aires, I9I2, tomo XIV, núm. 8 , p. 646.

Irocust destruction in the Cape Midlands. The Agricultural Journal of the Union of South Africa, Pretoria, I9I4, vol. VII, no. 5, pp. 706-708.

Locust (The) invasion of I889-92. Indian Mnseum Notes, Calcutta, I893, vol. III, no. 2, pp. $77-86$.

Locusts. Agricultural Gagette of Canada, Ottawa, I914, vol. I, no. 8, p. 627 .

Locusts. The Agricultural News, Barbados, I9I5, vol. XIV, no. 343, p. 202. 
[Locusts]. Bulletin of the Department of Agriculture, Trimdad and Tobago, Port-of-Spain, I9r6, vol. XV, part 4 , p. I43.

Locusts at Cedros. Department of Agriculture, Trinidad. Bulletin of Agriculturat Information, Port-of-Spain, I909, no 63, new series, p. 92.

Locnsts in Demerara. The A gricultural News, Barbados, I9I 5, vol. XIY, 110. 352, p. 347.

Locusts (The) of Bengal, Madras and Bombay. Indian Museum Notes, Calcutta, I8gi, rol. II, no. 4, pp. 99-II 5 .

Lucha (La) contra la langosta. Anales de la Sociedad Rural Argentina, Buenos Aires, Igr 5, año L, vol. XI IX, n. 6, p. 5 I 9 .

Iucha (La) contra la langosta. Revista de la Asociación Rural del Unuguay, Montevideo, IgI:. aก̃o XLV, núm. II , pp. $627-628$.

Lutte contre les sauterelles. Revue Horticole de l'Algérie, Alger, I9I6, t. XX, $20^{\mathrm{e}}$ an., $110 \mathrm{~s}$ I-2, pp. 31-34; Bulletın Agricole de l'Algárie-Tunisie-Maroc, Alger, I9I5 [I9I6], $2^{\mathrm{e}}$ série, 2I an., $\mathrm{n}^{0}$ 12, pp. 305-308.

Lutte (La) contre les sauterelles dans la Charente. Journal d'Agriculture pratique, Pari=, igos, nouv. série, $65^{\mathrm{e}}$ an., $2^{\mathrm{e}}$ sem., t. II, pp. r43-I44.

Mort (I,a) des sauterelles. Bullctin de l'Union des Agriculteurs d'Egypte, Le Caire, I9I \&, I2 an., no I03, pp. 59-60.

Movimiento de la langosta. Boletín del Ministerio de A gricultura, Buenos Aires, I912, tomo XIV, núms. II y I2, pp. I24I-I242, I244-I252; I9I3, tomo XV, núm. 5, p. 5I9; I9I4, tomo XVII, núm. 5, p. 493.

Note sur le criquet pèlerin (Schistocerca peregrina O1.). Tunis, I915, $20 \mathrm{pp}$., fig.

Nouveau dictionnaire d'histoire naturelle appliquée aux arts. Paris, De Crapelet-Lcterville, . I 803, t. XX, pp. I80-I87.

Peligro (El) de la langosta. Gaceta Rural, Buenos Aires, I9r6, año IX, núm. ro7̄, pp. 680 58I.

Perjuicios causados por la langosta. La Industria Pecuaria, Madrid, I9I6, añoXVII, núm. 522, pp. $665-666$.

Plagas de la agricultura. Boletín del Ministerio de Agricultura, Buenos Aires, I913, tomo XV, núm. I, p. 37; núm, 2, pp. r69-I7o; núm. 3, p. 260; núm. 4, pp. 357-358; núm. 5, p. 520.

Rapport phytopathologique pour an née I9I4. Annales du Service des Épiphyties, Paric, r9I6, t. III, p. Io.

Report on the prevalence of some pests and diseases in the West Indies during $\mathrm{rgr}_{4}$. West Indian Bulletin, Bridgetown, Barbados, I9I5, vol. XV, no. 2, pp. I24, I45.

Sauterelles (Lres) en Oranie. Revue Agricole ct Viticole de l'A frique du Nord, Alger, , 9I 4, t. III, no 1 I7, pp. $534-536$.

Summarising report on locust work for the second quarter, april ist to june 3 oth, I915. The Agricultural Bulletin of the Federated Malay States, Singapore, r915, vol. IV, no. I, pp. I3-20.,

30-foot hopperdozer. Details of construction of implement designed for large fielts, and uneven ground. U. S. Department of Agriculture. Weekly News Letter, Washington, D. C., I9I 5, vol. III, no. II, p. 5 .

To control grasshopper outbreaks. Freeman's Farmer, North Yakima, Wash., rgr6, rol. 7o, no. 6, p. 8 .

Utmntatás a marokkói sáska irtására cziprusi sövenynyel. Budapest, I89o, I2 pp., fig.

Vaquinhas (As) e sua destruição. A Evoluçào Agricola, São Paulo, IgII, ammo II, num. SXIII pp. 9-14. 


\section{TABLE DES MATIÈRES}

I. - HISTORIQUE ET DISTRIBUTION GÉOGRAPHIQUE DES SAUTERELLES. . I

II. - ESPÈCES DE SAUTERELLES NUISIBLES, OBSERVÉES DANS LES DIVERS PAYS CONSIDÉRÉS . . . . . . . . . . . . . . . . 39

III. - BIOLOGIE E'T MGEURS DES SAUTERELIES . . . . . . . . . . . . 5 I

Données sur le cycle évolutif des différentes espèces . . . . . . . . . . 5 I

Mœurs générales des sauterelles............... . 68

Modalités des apparitions et des migrations des diverses espèces dans les différents pays...................... 7 I

Causes qui déterminent la formation des bandes, les migrations et la multiplication des sauterelles................... $8_{4}$

Nourtiture ................ . . . 85

IV. - ORganisation EN VIGUEUR DANS LES DIVERS PAYS EN VUE DE LA LUTTE CONTRE LES SAUTERELLES. . . . . . . . . . . . . . . 99

Mesures législatives et administratives . . . . . . . . . . . . . . 99

Conventions internationales............... II3

Moyens financiers ................. . . II4

Moyens et initiatives privés ................ . . II9

V. - Moyens de LUTte EN USAgE Dans les Divers Pays. . . . . . . 123

Moyens naturels.................. I23

Moyens mécaniques et physiques............. . . I27

Moyens chimiques.................. I . I47

VI. - UTILITÉ D'UNE ENTENTE INTERNATIONALE POUR IA LUTTEE CONTRE LES SAUTERELLES . . . . . . . . . . . . . . . . . . . . I59

INDEX BIBLIOGRAPHIQUE. . . . . . . . . . . . . . . . . . . . . . ${ }^{6} 63$ 

d) Publications du Bureau des Institutions Économiques et Sociales.

1. L'ACTIVITÉ DE L'INSTTTUT INTERNATIONAC D'AGRICULTURE DANS LE DOMAINE DE LA COOPÉRATION, DE L'ASSTURANCE ET DU CREDIT AGRICOLES (en français, en allemand et en italien, 1912, 34 pages, in-16) . . . . . . . .

2. ÉTUDES MONOGRAPHIQUES SUR LA COOPÉRATION AGRICOLE DANS QUELQUES PAYs (Tome I) (en français 457 pages et en anglats 451 pages, 191 r, in-16) . IDEM (Tome II)) (en tranfais, 238 pages, 1914, in-16) . . . . . . IDEM (Tome II) (en anglais, $2 \times 3$ pages, Igr5, in-16). . . . . . .

3. AN OUTLINE OP THE EUROPEAN CO-Operattve CredT Systems (2 eme édition,

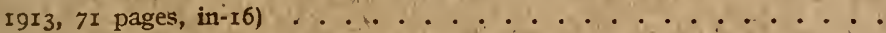

4. L'ORGANISATION DE LA STATISTIQUE DE LA COOPÉRATION AGRICOLE DANS QUEL-

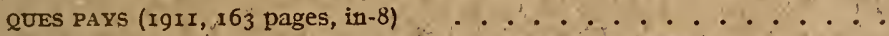

5. L'ASSURANCE-GRELE DANS QUELQUES PAYS ET SES PROBLEMES (IgII, I IO pages,

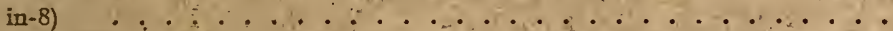

6. AGRICULTURAL CREDIT AND CO-OPERATION IN ITALY : SHORT GUTDE TO RURAL CO-OPERATION IN ITALY (en anglais 35 pages et en italien, 34 pages, in-I6) .

e) Autres publications.

I. L'INSTITUT INTERNATIONAL D'AGRICULTURE, SON ORGANISATION, SON ACTIVITÉ, SES RÉSULTATS (illustré, I $9 \mathrm{r}_{4}$ : en français, en italien, et en anglais : 45

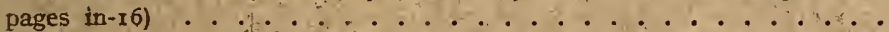

2. Lovis-Dop. - Le présent et l'avenir de l'Institut International d'Agriculture. Conférence (rgI2, 60 pages in-I6) . . . . . . ...

3. Santiago aldunate. - El Instituto Internacional de Agricultura y su importancia para la América Lratina, en especial para Chile. Conférencia. (1913,

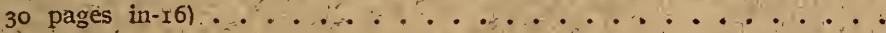

fr. $\quad 1,00$

1,00

- $\quad \mathrm{I}, 00$

\section{PUBLICATIONS NE SE TROUVANT PAS DANS LE COMMERCE.}

I. CONFÉRENCE INTERNATTONALE DE IgO5 POUR LA CRÉaTION D'UN INSTTTUT INTERNATTONAL D'AGRICULTURE (1905, 254 pages in-4).

2. ACTES DES ASSEMBLEES GÉNÉRALES DES ANNÉES I908, Ig09, I9II, Igr3 (trois volumes in-8, un in-16).

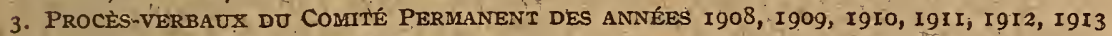
Igr4 et IgI 5 (cinq volumes in-8, trois in-I6).

4. RAPPORTS ET ETUDES DO BUREAU DE LA STATTSTTQUE GÉNÉRALE (I9II, 260 pages, in-8).

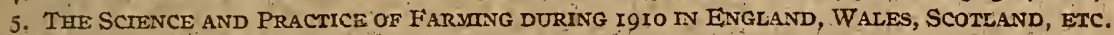
(1910, 646 pages, in-16).

6. ETUDE SUR LES RECENSEMENTS DE LA POPULATION AGRICOLE, LES SACATRES DE LA MAIND'CEUVRE, RURALE ET LES COURANTS D'EMIGRATION DANS LES DIFFÉRENTS ÉTATS (IgI2, I50 pages in-8).

\section{AVIS.}

I) Pour les abonnements et l'achat des publications de 1'Institut International d'Agrtculture, on peut s'adresser directement à í

\section{Institut Intemational d'Agriculture \\ Service des abonnements et des prblications \\ Villa Umberto $I$ \\ ROME (Italie).}

2) Le prix des publications est franco port et emballage pour l'Italie et l'étranger. Toutes les publications sont expédiées sur le simple envoi d'un mandat-poste ou d'un couponréponse intènational.

3) Les expéditions sont faites avec soin et ponctuellement, mais les volumes non recommandés sont aux risques du client.

4) Pour recevoir les livres par colis recommandé, afin d'éviter des pertes, dont l'Institut International d'Agriculture ne peut prendre la responsabilite, on est, prié d'ajouter o fr. 25 centimes.

5) En cas de changement d'adresse, on est prié d'envoyer à l'Institut International d'Agriculture la barde d'abonnement portant les corrections nécessaires, en l'adressant au Ser. vice des abornements et des publications.

Pour la vente et les abonnements en France et dans les Colonies françaises on peut s'adresser à 








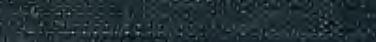

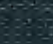

28

tion

然

Hen

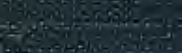

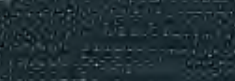

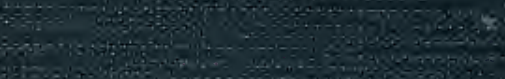

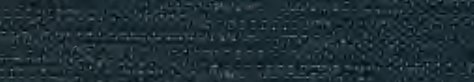

and

(1)

1.

Hexply

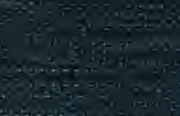

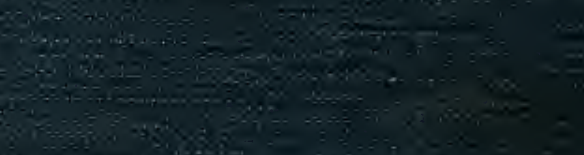

1.4.

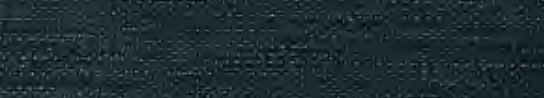

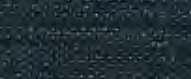

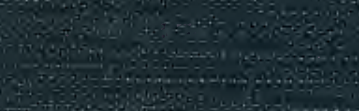

1.5.

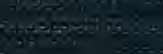

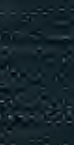

